\title{
CHARACTERIZATION OF DIRECT ADDITIVE MANUFACTURED U3Si2 SURROGATE TO PREDICT U3Si2 MICROSTRUCTURES
}

\author{
JHONATHAN F. ROSALES FRANCO \\ May 2018
}

The INL is a U.S. Department of Energy National Laboratory operated by Battelle Energy Alliance

Idaho National Laboratory 


\section{CHARACTERIZATION OF DIRECT ADDITIVE MANUFACTURED U3Si2 SURROGATE TO PREDICT U3Si2 MICROSTRUCTURES}

JHONATHAN F. ROSALES FRANCO

May 2018

Idaho National Laboratory Idaho Falls, Idaho 83415

http://www.inl.gov

Prepared for the

U.S. Department of Energy

Under DOE Idaho Operations Office

Contract DE-AC07-05ID14517 
CHARACTERIZATION OF U $\mathrm{Si}_{2}$ SURROGATES TO PREDICT U $\mathrm{Si}_{2}$ ADDITIVE MANUFACTURED MICROSTRUCTURES

By

JHONATHAN ROSALES

A DISSERTATION PRESENTED TO THE GRADUATE SCHOOL OF THE UNIVERSITY OF FLORIDA IN PARTIAL FULFILLMENT

OF THE REQUIREMENTS FOR THE DEGREE OF DOCTOR OF PHILOSOPHY

UNIVERSITY OF FLORIDA 
(C) 2018 Jhonathan Rosales 
"I am dedicating this dissertation to my parents for their love and support. Also, to my siblings Jenny, Herber, and Javier for motivating me to achieve my personal goals. Without you, this would not have been possible." 


\section{ACKNOWLEDGMENTS}

This research work was sponsored by the U.S. Department of Energy, Office of Nuclear Energy, under U.S. Department of Energy Idaho Operations Office Contract DE-AC0705ID14517, as part of the Technology Commercialization Fund and Energy I-Corps Cohort 5.

The research work was performed as part of the Nuclear Science and Technology

Division, Idaho National Laboratory, with Dr. Isabella J van Rooyen as the principle investigator (PI) and inventor of the Technology Commercialization Fund project (Additive Manufacturing as an Alternative Fabrication Technique for the Fabrication of Uranium Silicide Fuel (AMAFT) and Energy I-Corps Cohort 5 projects, and Dr. Clemente Parga as the co-PI and co-inventor of the Technology Commercialization Fund project.

The experimental work was performed at the INL Research Center (IRC) laboratories and the characterization was performed at the Center for Advanced Energy Studies (CAES). Additional contributors to the $\mathrm{U}_{3} \mathrm{Si}_{2}$ AMAFT Development and Energy I-Corps Projects are: David Swank (INL), DC Haggard (INL), George Griffith (INL), and Ed Lahoda (Westinghouse Electric). Additional support during the sample preparation and electron microscopy was provided by Brian Forsman (CAES: Boise State University), Jatu Burns (CAES: Boise State University) and Yaqiao Wu (CAES: Boise State University).

Many thanks to Jim Tulenko (UF), Clemente Parga (INL), Subhashish Meher (INL), Jason Harp (INL), Rita Hoggan (INL) and Manny Perez (INL) for their technical advice and contributions. And special thanks to Isabella van Rooyen (INL) for her mentoring and guidance as a principal study supervisor in this project. 


\section{TABLE OF CONTENTS}

page

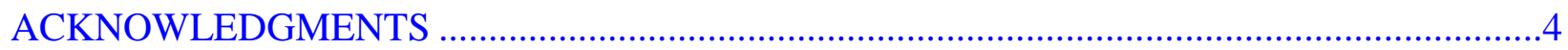

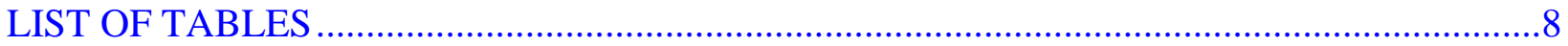

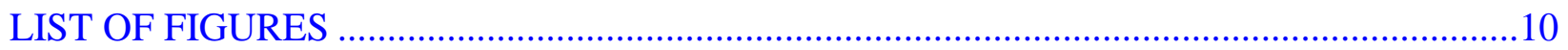

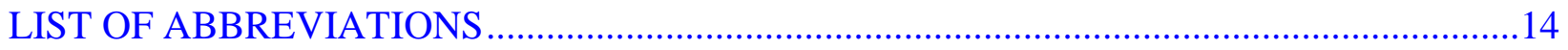

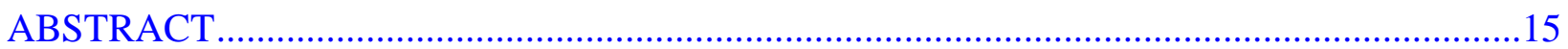

CHAPTER

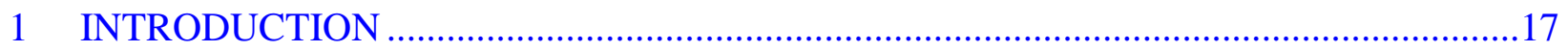

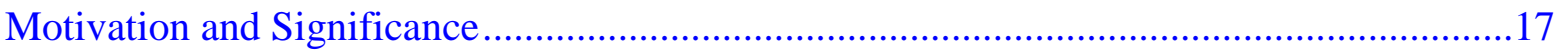

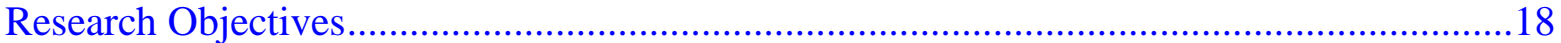

Objective 1 - A Thermodynamic Analysis - Understanding Surrogate Fuel

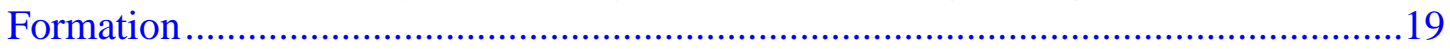

Objective 2 - Effects of Laser Interaction on $\mathrm{U}_{3} \mathrm{Si}_{2}$ Surrogates - Characterization........20

Objective 3 - A Prediction of the Formation of $\mathrm{U}_{3} \mathrm{Si}_{2}$ based on Surrogate

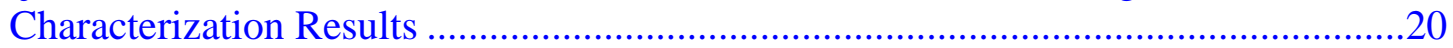

INL Long Term Goal: Development of the AMAFT Process ..........................................21

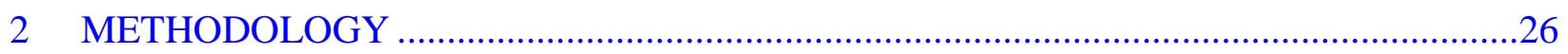

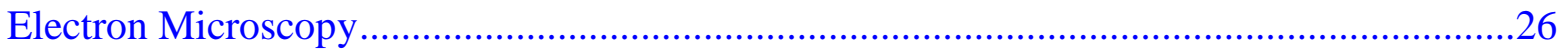

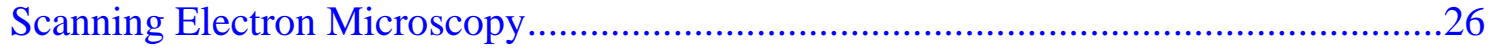

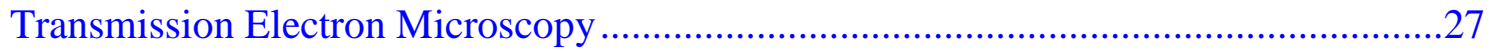

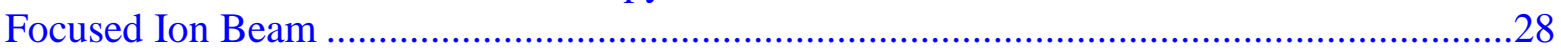

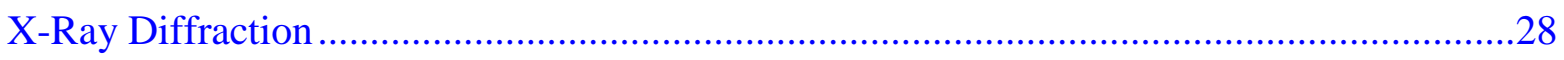

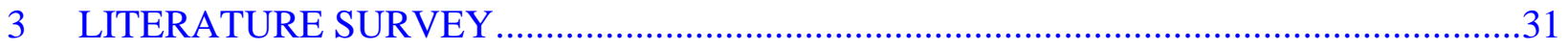

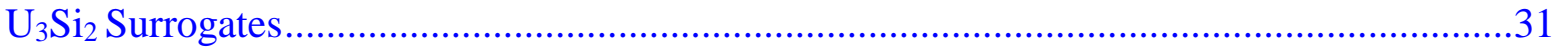

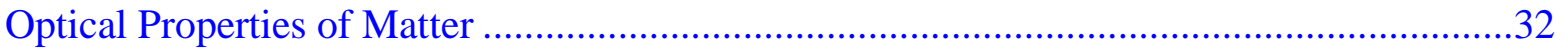

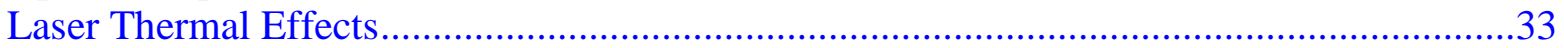

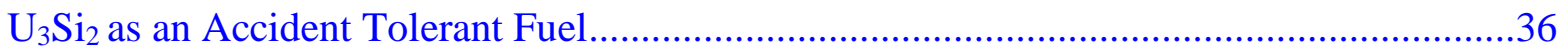

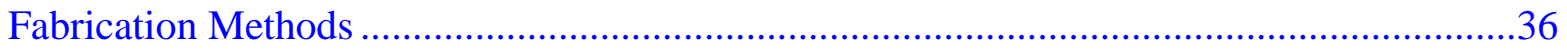

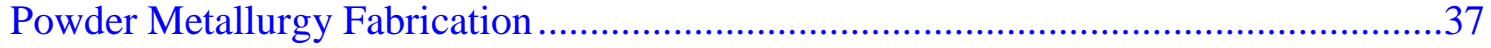

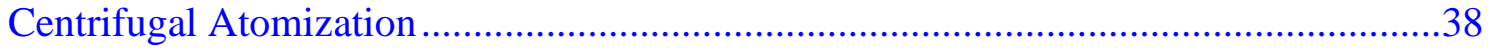

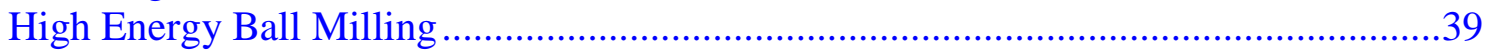

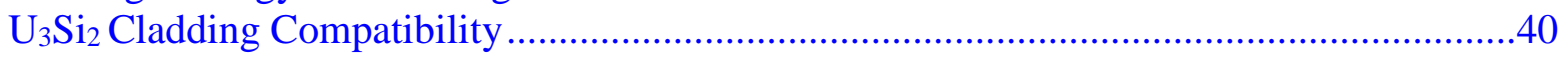

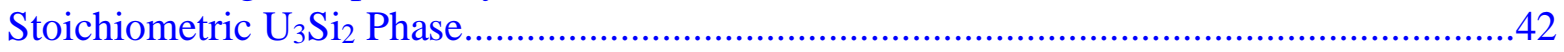

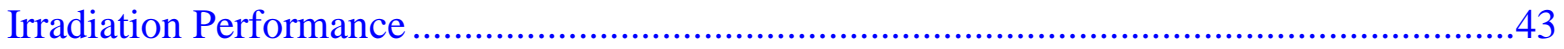




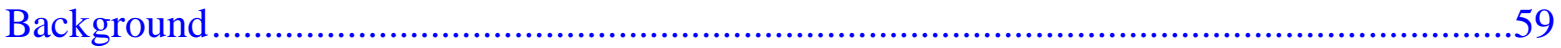

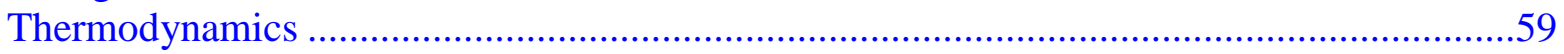

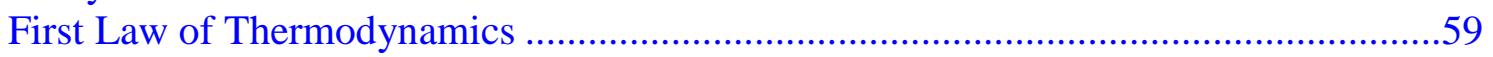

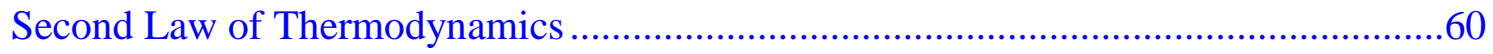

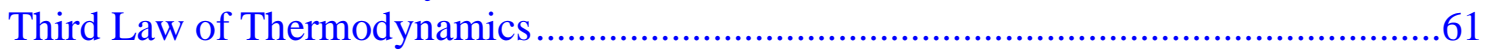

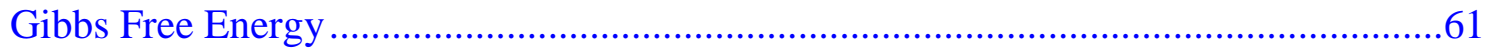

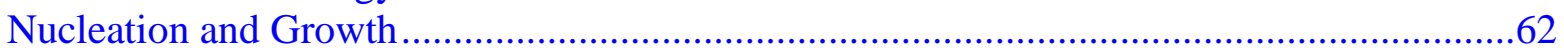

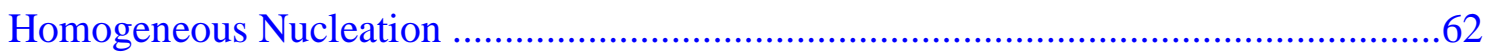

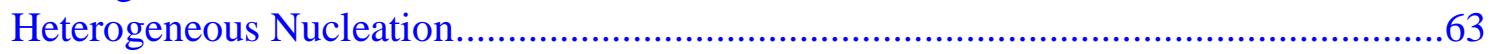

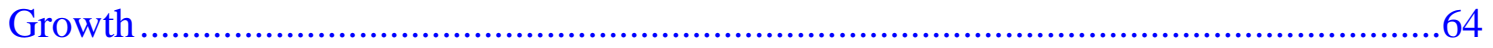

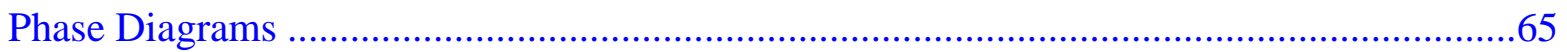

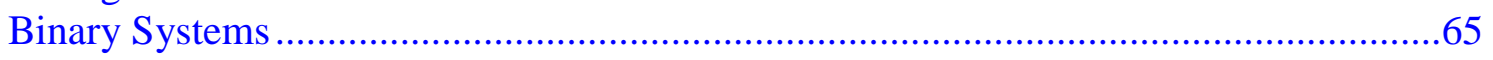

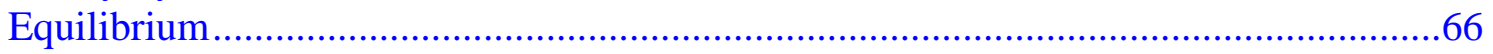

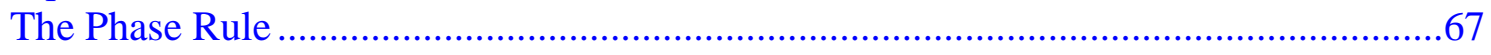

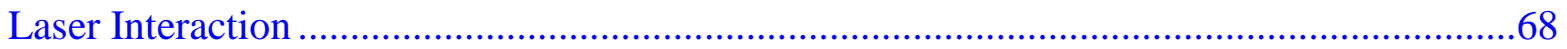

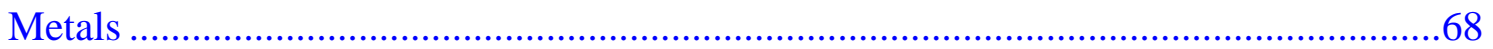

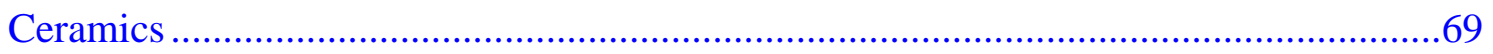

Phase Diagram Analyses - Uranium and Surrogate Materials...........................................70

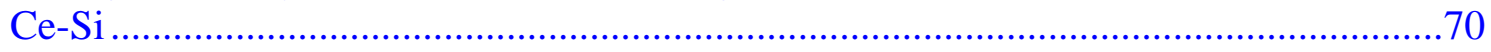

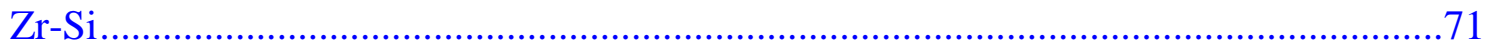

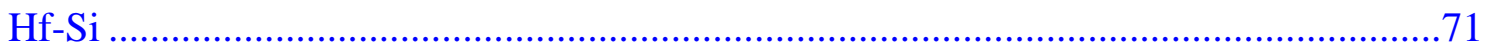

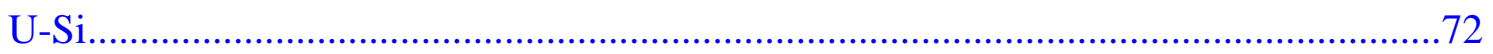

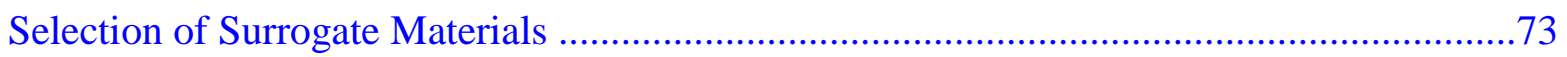

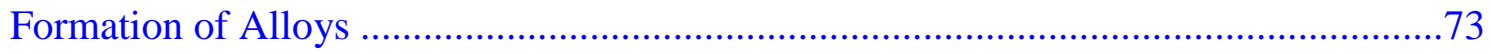

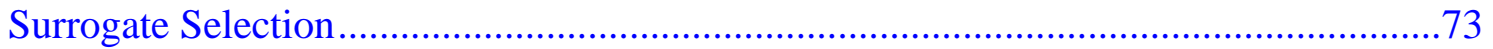

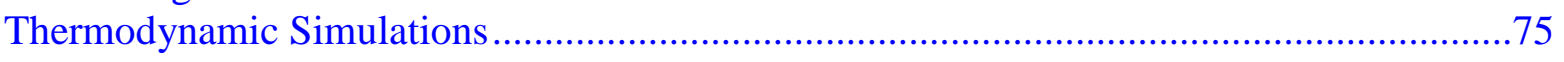

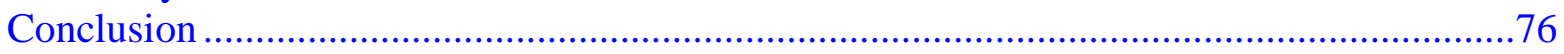

5 EFFECTS OF LASER INTEARCTION ON U $\mathrm{Si}_{2}$ SURROGATES -

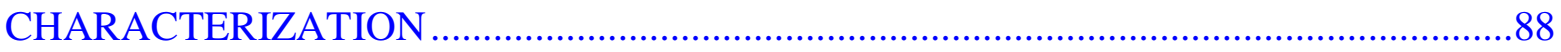

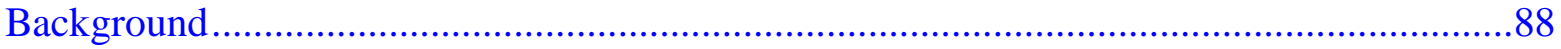

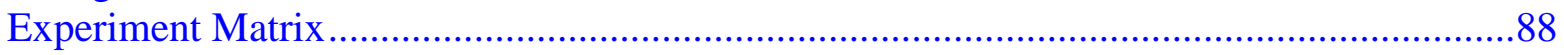

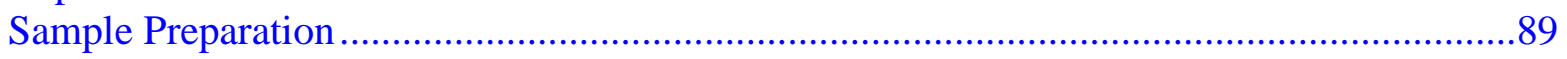

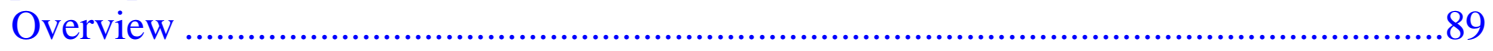

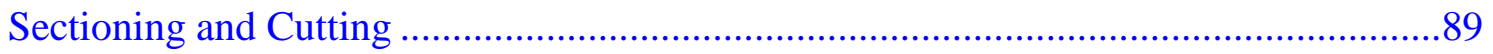

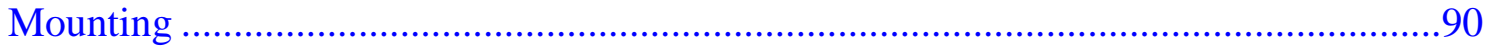

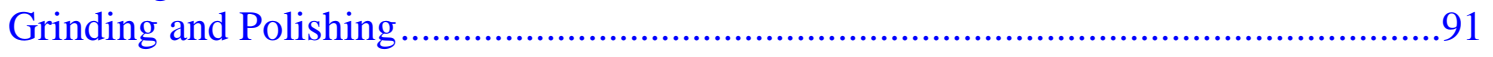

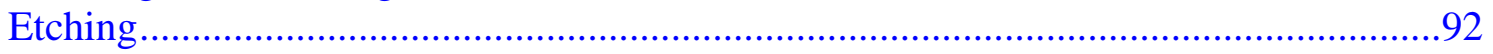

Preliminary Laser Synthesis of Surrogate Materials ..................................................92

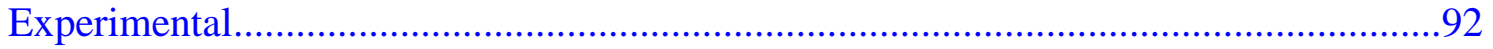

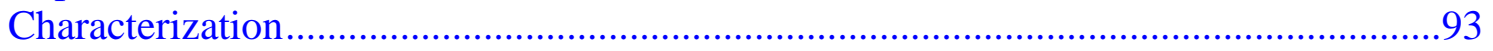

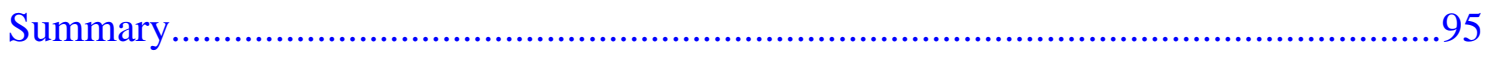

Characterization of Downselected Surrogates ............................................................95 
$\mathrm{Ce}-\mathrm{Si}$

$\mathrm{Zr}-\mathrm{Si}$

$\mathrm{Hf}-\mathrm{Si}$

100

Conclusion

6 A PREDICTION OF THE FORMATION OF $\mathrm{U}_{3} \mathrm{Si}_{2}$ BASED ON SURROGATE CHARACTERIZATION RESULTS.

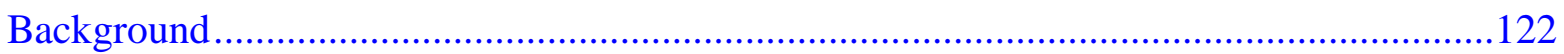

Characterization of $\mathrm{U}_{3} \mathrm{Si}_{2}$ Powder Metallurgical Route ..................................................122

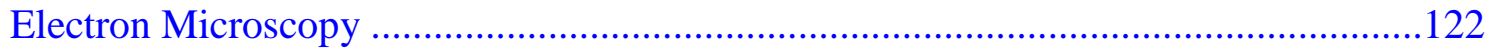

Scanning electron microscopy and energy dispersive spectroscopy .......................122

Transmission Electron Microscopy ………………………………………….....124

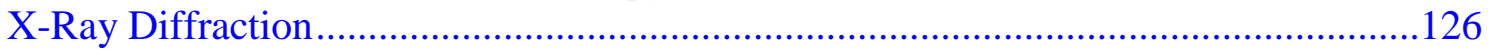

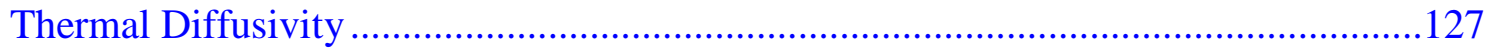

Effect of High Si-Content on $\mathrm{U}_{3} \mathrm{Si}_{2}$ Fuel Microstructure ...................................................128

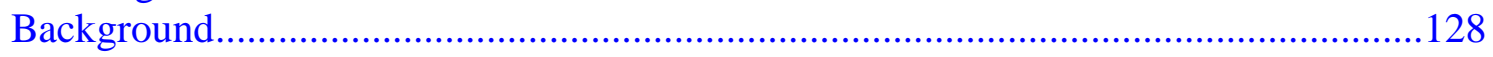

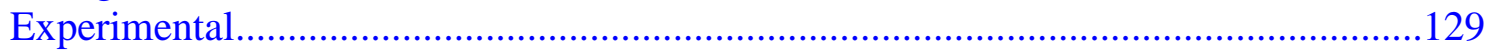

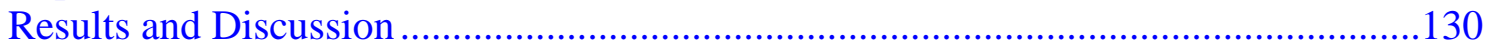

TEM

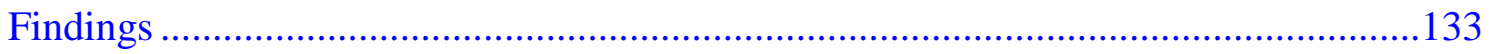

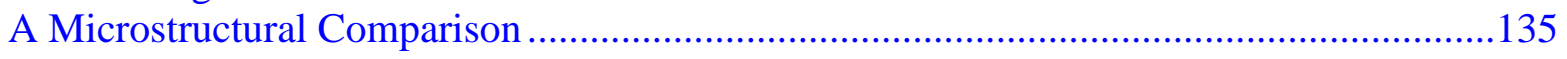

Prediction of $\mathrm{U}_{3} \mathrm{Si}_{2}$ Microstructure During a Laser Synthesis .............................................137

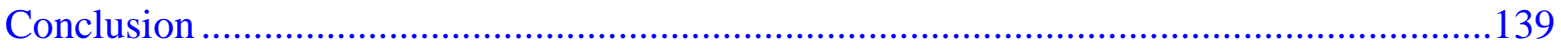

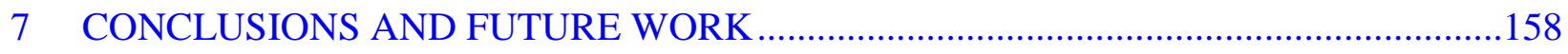

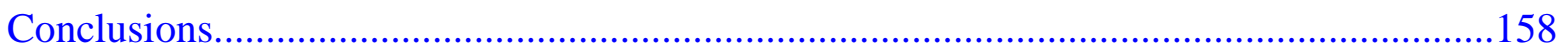

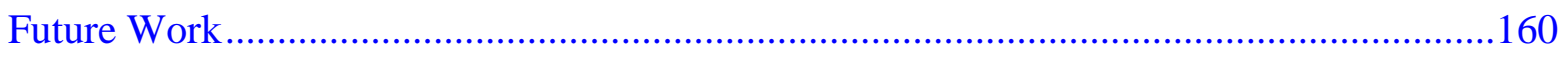

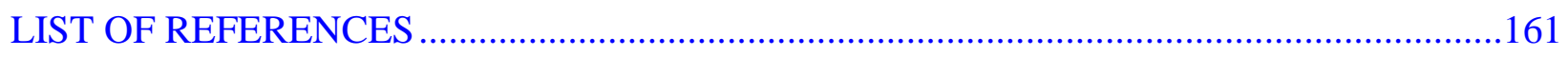

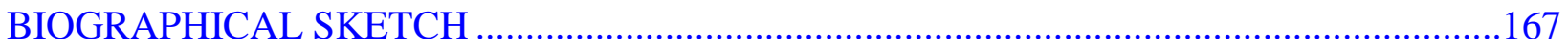




\section{LIST OF TABLES}

$\underline{\text { Table }}$

page

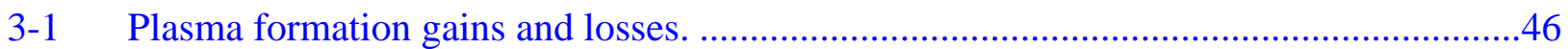

3-2 Density of different accident-tolerant fuel candidates. .............................................46

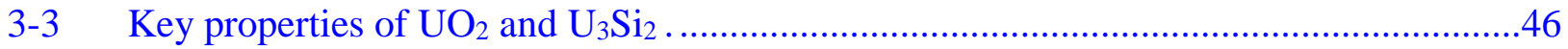

3-4 Crystallographic and thermodynamic data for the U-Si system. ..............................47

3-5 Chemical analysis conducted on comminuted $\mathrm{U}_{3} \mathrm{Si}_{2}$ buttons.................................47

4-1 Property list of thermodynamic and mechanical properties of selected compounds........78

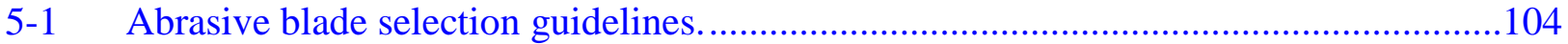

5-2 Diamond wafer blade selection guidelines ....................................................... 104

5-3 Compression mounting application properties. .................................................... 105

5-4 Compression mounting resin physical properties. ..................................................105

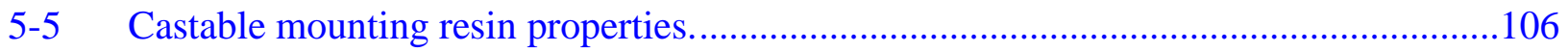

5-6 Particle size vs. common grit sizes for abrasive papers..........................................106

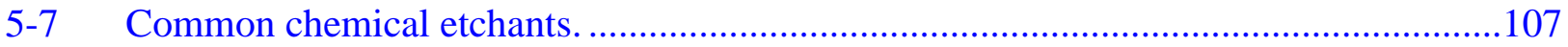

5-8 Thermo-physical properties of elements at room temperature. ...............................108

5-9 EDS quantification analysis on preliminary laser synthesis stage employing

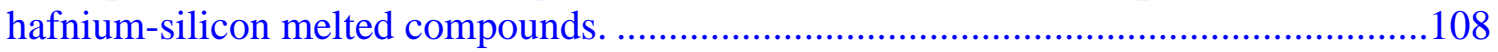

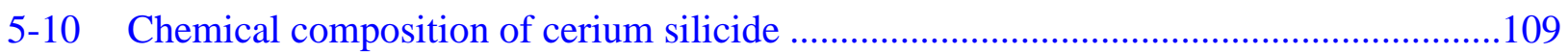

5-11 EDS quantification analyses on zirconium-silicon melted compounds.......................109

5-12 EDS quantification analyses on hafnium-silicon melted compounds..........................110

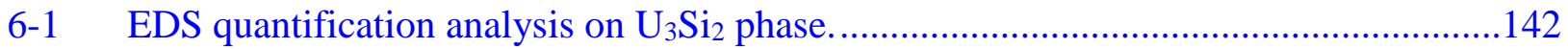

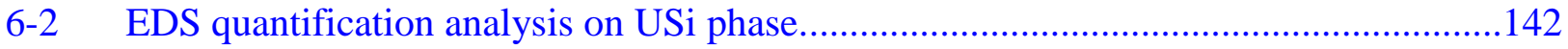

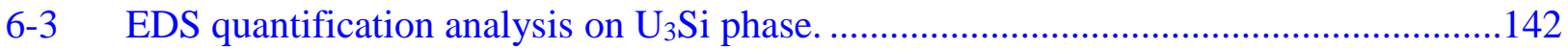

6-4 EDS quantification analysis on TEM generated on $\mathrm{U}_{3} \mathrm{Si}_{2}$ samples ..........................143 
6-5 Key manufacturing parameters for $\mathrm{U}_{3} \mathrm{Si}_{2}$ pellets used in this study.

6-6 Phase quantification results from digital image analysis...............................................143 


\section{LIST OF FIGURES}

Figure $\quad$ page

1-1 Potential compounds of the fuel cycle that will be utilized to produce $\mathrm{U}_{3} \mathrm{Si}_{2} \ldots \ldots \ldots \ldots \ldots . . .23$

1-2 Optimization scheme of the novel additive manufacturing process to produce $\mathrm{U}_{3} \mathrm{Si}_{2} \ldots . . .23$

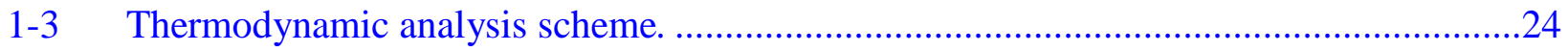

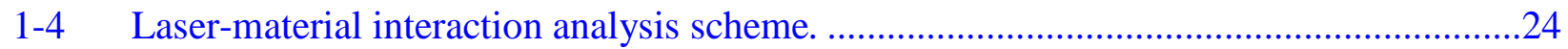

1-5 Characterization outline comparison of surrogate microstructure ...............................25

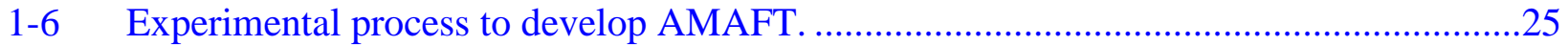

2-1 Characterization equipment employed in this research...........................................30

3-1 Microstructural SEM images from $\mathrm{Ce}_{3} \mathrm{Si}_{2}$ synthesis.. ............................................48

3-2 Typical spark plasma sintering experiment conducted with as-milled $\mathrm{Ce}_{3} \mathrm{Si}_{2}$ powder. ....48

3-3 SPS sintered $\mathrm{Ce}_{3} \mathrm{Si}_{2}$ displaying a few small, rounded pores at grain boundaries. ............49

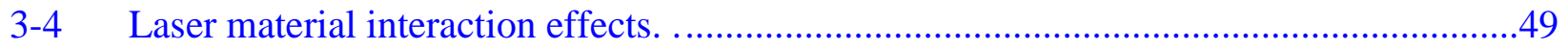

3-5 Second harmonic generation process of incident beam of frequency.............................50

3-6 The laser energy damage generated in a polycrystalline silicon target.. .........................50

3-7 $\mathrm{U}_{3} \mathrm{Si}_{2}$ fuel fabrication process on a laboratory scale. .............................................51

3-8 Electron backscatter image of $\mathrm{U}_{3} \mathrm{Si}_{2}$ inclusions in arc melted uranium silicide..............51

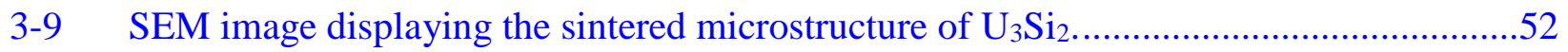

3-10 A schematic view of the centrifugal atomizer apparatus ..........................................52

3-11 Void content of extruded fuel cores with comminuted and atomized $\mathrm{U}_{3} \mathrm{Si}_{2}$ particles......53

3-12 Representative micrographs from the specified milling times. ..................................53

3-13 Representative XRD $2 \theta$ scans for $\mathrm{U}_{3} \mathrm{Si}_{2}$ milled in clean vessels with CSZ media for

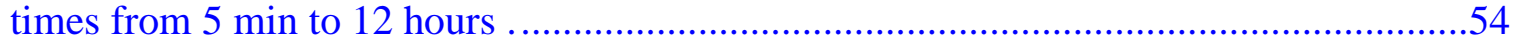

3-14 Thermal conductivity of fuel and cladding materials. ............................................54

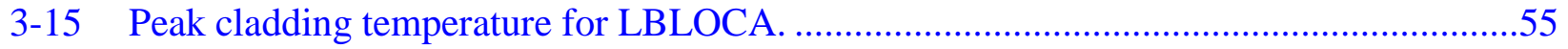




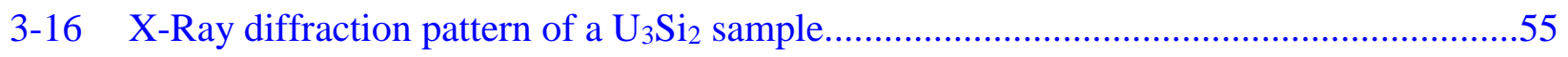

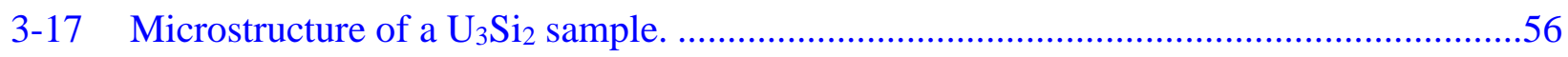

3-18 Fuel particle swelling as a function of fission density....................................................56

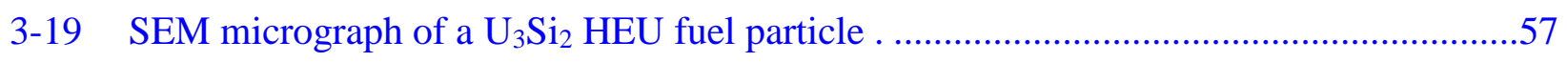

3-20 Nuetron irradiated uranium-silicide samples................................................................57

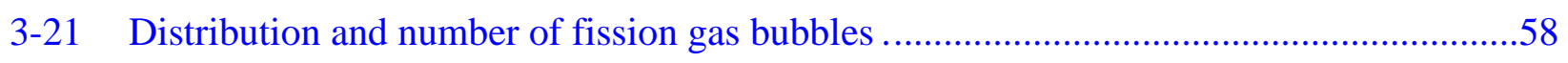

4-1 Diagram displaying the nucleation of a spherical solid particle in a liquid........................80

4-2 Plot of free energy versus embryo/nucleus radius, displaying the critical free energy

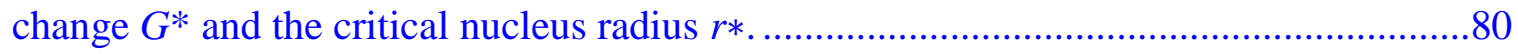

4-3 Heterogeneous nucleation of a solid from a liquid. .......................................................

4-4 Plot representing curves for homogeneous and heterogeneous nucleation. The critical energies and critical radii are displayed.........................................................................

4-5 Plot displaying nucleation rate $(\dot{\mathrm{N}})$, growth rate $(\dot{\mathrm{G}})$ and total transformation versus

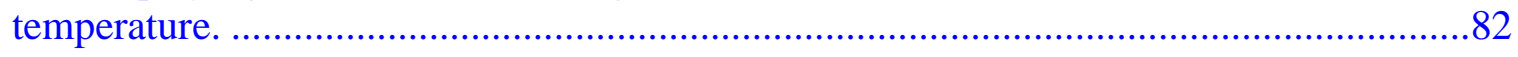

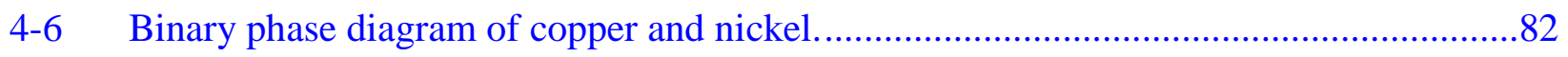

4-7 Block diagram of material removal process by means of laser energy interaction. ...........83

4-8 Ratios of the absorption of rough and smooth surfaces for normally incident light shown as a function of slope roughness for selected metals.............................................83

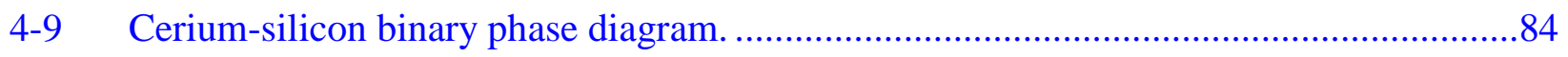

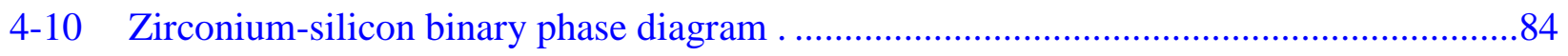

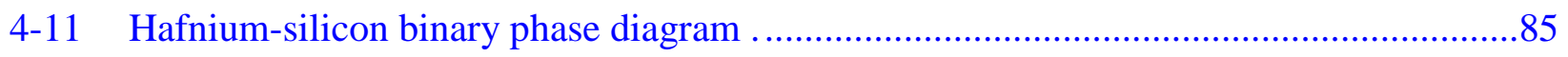

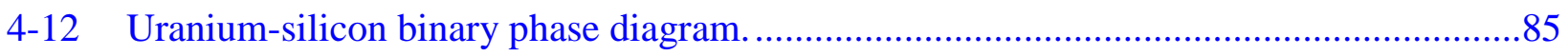

4-13 ThermoCalc simulation results on Gibbs free energy of $\mathrm{Zr}_{3} \mathrm{Si}_{2}$ with respect to temperature at $100,000 \mathrm{~Pa}, 0.1 \mathrm{~Pa}$, and $0.0001 \mathrm{~Pa}$..

4-14 ThermoCalc simulation results on Gibbs free energy of $\mathrm{Hf}_{3} \mathrm{Si}_{2}$ with respect to

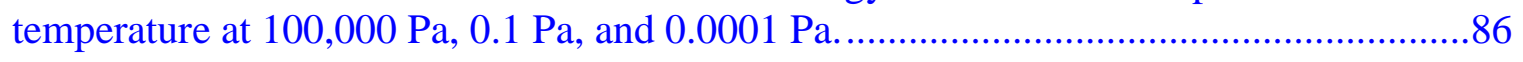

4-15 ThermoCalc simulation results on Gibbs free energy of $\mathrm{Hf}_{3} \mathrm{Si}_{2}$ with respect to temperature at $100,000 \mathrm{~Pa}, 0.1 \mathrm{~Pa}$, and $0.0001 \mathrm{~Pa}$. 
5-1 Optical microscopy images of preliminary experiments of the AMAFT process.

5-2 SEM micrographs of preliminary experiments of the AMAFT process........................111

5-3 XRD pattern collected on a hafnium silicide compound. ...........................................112

5-4 SEM micrograph displaying the morphology of a hafnium silicide compound from which EDS analyses were collected on different locations. ......................................112

5-5 SEM micrographs displaying the microstructure of laser synthesized Ce-Si. ..............113

5-6 SEM micrograph displaying a spot on the cerium-silicon compound .........................114

5-7 XRD pattern collected on cerium-silicon compound.............................................114

5-8 XRD pattern collected on cerium-silicon powder. ...............................................115

5-9 SEM images of zirconium silicide fabricated by laser synthesis..............................116

5-10 SEM micrographs of laser synthesized Zr-Si. ................................................117

5-11 XRD pattern collected on Zr-Si Sample SZ-S1 ..............................................117

5-12 XRD pattern collected on Zr-Si Sample SZ-S2 ..................................................118

5-13 SEM micrographs of hafnium silicide fabricated by laser synthesis..........................119

5-14 SEM micrograph displaying locations where XRD readings were collected on hafnium-silicide Sample HS-S1 ....................................................................... 120

5-15 XRD analysis on laser synthesis of cerium-silicon compound.................................120

5-16 XRD analysis on laser synthesis of cerium-silicon compound.................................121

6-1 SEM images displaying the microstructure of $\mathrm{U}_{3} \mathrm{Si}_{2}$ fabricated by means of a powder metallurgical process.

6-2 Electron backscatter micrographs displaying areas with high concentration of $\mathrm{U}_{3} \mathrm{Si}_{2}$ confirmed by EDS

6-4 Electron backscatter micrographs displaying areas with high concentration of $\mathrm{U}_{3} \mathrm{Si}$ confirmed by EDS.

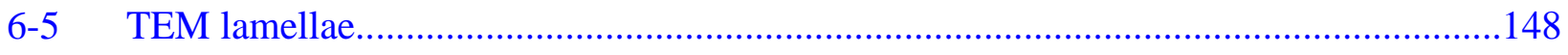

6-6 TEM micrographs where chemical analyses were performed on $\mathrm{U}_{3} \mathrm{Si}_{2}$ samples..... 149

6-7 SEM micrographs of $\mathrm{U}_{3} \mathrm{Si}_{2}$ samples where $\mathrm{XRD}$ analyses where performed...............150 
6-8 XRD analysis of $\mathrm{U}_{3} \mathrm{Si}_{2}$ Sample A2.

6-9 XRD analysis of $\mathrm{U}_{3} \mathrm{Si}_{2}$ Sample D4.

6-10 XRD analysis of $\mathrm{U}_{3} \mathrm{Si}_{2}$ Sample C.

6-11 Thermal diffusivity of $\mathrm{U}_{3} \mathrm{Si}_{2}$ samples as a function of temperature

6-12 Back scatter SEM images of $\mathrm{U}_{3} \mathrm{Si}_{2}$ samples.

6-13 XRD analysis of Samples A2 and C

6-14 Images displaying $\mathrm{SAD}$ results on $\mathrm{U}_{3} \mathrm{Si}_{2}$ samples.

6-15 Images displaying $\mathrm{SAD}$ results on $\mathrm{U}_{3} \mathrm{Si}_{2}$ samples.

6-16 SEM images comparing the morphology of $\mathrm{U}_{3} \mathrm{Si}_{2}$ and $\mathrm{Zr}_{3} \mathrm{Si}_{2}$ compounds .156

6-17 SEM micrographs comparing the morphology of $\mathrm{Hf}_{3} \mathrm{Si}_{2}$ and $\mathrm{U}_{3} \mathrm{Si}_{2}$ samples. 157 


\section{LIST OF ABBREVIATIONS}

\begin{tabular}{ll} 
AMAFT & Additive Manufacturing as an Alternate Fabrication Technique \\
BDRF & Bi-directional Reflection Function \\
BSU & Boise State University \\
CAES & Center for Advanced Energy Studies \\
EBSD & Electron Backscatter Diffraction \\
EDS & Energy Dispersive Spectroscopy \\
FIB & Focused Ion Beam \\
HEBM & High Energy Ball Milling \\
HEU & High Enriched Uranium \\
INL & Idaho National Laboratory \\
LBLOCA & Large Break Loss of Coolant Accident \\
MSR & Mechano-chemically-Induced Self-Propagating Reaction \\
PWR & Pressurized Water Reactor \\
SEM & Scanning Electron Microscopy \\
SiC & Silicon Carbide \\
SPS & Spark Plasma Sintering \\
TEM & Transmission Electron Microscopy \\
TD & Theoretical Density \\
UF & University of Florida \\
UO & Uranium Dioxide \\
HSi $_{3}$ & Uranium Sesquisilicide \\
XRD & X-Ray Diffraction \\
\hline
\end{tabular}




\section{Abstract of Dissertation Presented to the Graduate School of the University of Florida in Partial Fulfillment of the Requirements for the Degree of Doctor of Philosophy \\ CHARACTERIZATION OF U $\mathrm{Si}_{2}$ SURROGATES TO PREDICT U $\mathrm{S}_{3} \mathrm{Si}_{2}$ ADDITIVE MANUFACTURED MICROSTRUCTURES}

\section{By}

Jhonathan Rosales

May 2018

Chair: Isabella van Rooyen

Major: Nuclear Engineering Sciences

The purpose of this research is to perform characterization exclusively on $\mathrm{U}_{3} \mathrm{Si}_{2}$ surrogate materials to predict the process envelope for future experiments with uranium compounds. The surrogate material is synthesized employing a laser energy source. This characterization work provides new property data to aid the concept development stages of fabricating $\mathrm{U}_{3} \mathrm{Si}_{2}$ by means of a novel direct additive manufacturing methodology (AMAFT process) invented at the Idaho National Laboratory. This process not only seeks to potentially reduce production costs, but also to shorten the current silicide fuel fabrication process. $\mathrm{U}_{3} \mathrm{Si}_{2}$ has been down selected as a candidate for accident-tolerant fuel for commercial light-water reactors due to its improved thermal conductivity, higher atomic density, and fuel cycle economics when compared with $\mathrm{UO}_{2}$. The research efforts on this work are performed at INL in partnership with Westinghouse Electric $®$.

The initial stage of the surrogate research includes a thermodynamic study along with physical and mechanical property analyses to assist in the down selection of surrogate materials for initial experimentation. Initial characterization analyzed the microstructural effects to determine effectiveness of laser interaction on the surrogate materials followed by detailed 
characterization via scanning electron microscopy (SEM), transmission electron microscopy (TEM), and X-ray diffraction (XRD). Complementary comparative studies were performed on the surrogate end products, analyzing similarities in microstructural features present on $\mathrm{U}_{3} \mathrm{Si}_{2}$ fuel fabricated by means of alternate methods. Novel microstructural data on surrogate silicide fuel fabricated by means of the AMAFT process (laser synthesis) was crucial to confirm the formation of surrogate silicide fuels $\left(\mathrm{Hf}_{3} \mathrm{Si}_{2}\right.$ and $\left.\mathrm{Zr}_{3} \mathrm{Si}_{2}\right)$. From the selected surrogates, $\mathrm{Zr}_{3} \mathrm{Si}_{2}$ was the most relevant for this work. Besides its successful trajectory as a uranium surrogate for research purposes, $\mathrm{Zr}_{3} \mathrm{Si}_{2}$ can also be incorporated in different applications within the nuclear industry (ie. Gen IV reactor reflector). These unique characterization results from the surrogate compounds are available for future laser based experimental work and will provide a data base for future uranium surrogate choices. The results of this research assisted in the prediction of the end product morphology when working with uranium compounds and highly supported the development of the AMAFT process. Upon completion, the AMAFT process envisages to shorten the fabrication of $\mathrm{U}_{3} \mathrm{Si}_{2}$ fuel and ideally provide clean and reliable energy to benefit human kind. 


\section{CHAPTER 1 \\ INTRODUCTION}

\section{Motivation and Significance}

The purpose of this research is to perform characterization exclusively on $\mathrm{U}_{3} \mathrm{Si}_{2}$ surrogate materials to predict the process envelope for future work with uranium compounds. The characterization results will provide novel property data to aid the concept development stages of fabricating $\mathrm{U}_{3} \mathrm{Si}_{2}$ by means of a new additive manufacturing methodology. The characterization data is crucial to decide the readiness of experimentation and development for interim and final experiment design. $\mathrm{U}_{3} \mathrm{Si}_{2}$ fuel possess enhanced thermal properties, higher atomic density, and improved irradiation stability when compared to $\mathrm{UO}_{2}$. Due to the aforementioned advantages, $\mathrm{U}_{3} \mathrm{Si}_{2}$ was down selected as an accident tolerant fuel candidate.

Uranium silicide obtained by conventional arc or induction melting normally poses the challenge of achieving a pure $\mathrm{U}_{3} \mathrm{Si}_{2}$ phase. Studies have shown that, during the melting process, the formations of different phases alter the fuel's composition. The experimentally measured compositions of $\mathrm{U}_{3} \mathrm{Si}$, USi, and $\mathrm{U}_{3} \mathrm{Si}_{2}$ — the three most U-rich phases in the U-Si system —are present in the compound. Most fabrication methods face the difficulty of achieving an exact stoichiometry of the sample in which only the pure $\mathrm{U}_{3} \mathrm{Si}_{2}$ phase is present [1]. In addition, the current fabrication methods involve a long, laborious preparation process.

The Additive Manufacturing as an Alternate Fabrication Technique (AMAFT) process was invented by INL to manufacture nuclear fuel (scheme in Figure 1-1). This method will employ a laser energy source to produce $\mathrm{U}_{3} \mathrm{Si}_{2}$ directly from $\mathrm{Si}$ and other compounds (e.g $\mathrm{UF}_{4}$, $\mathrm{UO}_{2}$ ) from the fuel cycle, without the need to reduce uranium to its metallic state. Figure 1-2 illustrates the stages of the optimized additive manufacturing process compared to the conventional methodology to fabricate nuclear fuel. The direct synthesis from $\mathrm{UF}_{4}$ to $\mathrm{U}_{3} \mathrm{Si}_{2}$ could 
provide significant savings in both production time and manufacturing costs. In future stages of the project, the AMAFT process envisages to employ a feed of metallic uranium along with elemental silicon, and generate the alloying process to produce $\mathrm{U}_{3} \mathrm{Si}_{2}$ by means of the direct additive manufacturing process, reducing the steps when compared to a conventional powder metallurgical fabrication process. Apart from secondary phases of the U-Si binary system and potential enhanced surface roughness, no other issues are currently expected. However, if additional development on the end product needs to be performed, it will be implemented as the time comes.

The AMAFT process holds a proprietary invention disclosure and a provisional patent filed in March 2017. The AMAFT process development is funded by the U.S. Department of Energy in collaboration with Westinghouse Electric $®$ under a Cooperative Research and Development Agreement (CRADA).

The current dissertation focuses on the synthesis of $\mathrm{U}_{3} \mathrm{Si}_{2}$ surrogates to provide a general understanding of chemical reactions and product formation prior to experiments intending to form uranium silicide $\left(\mathrm{U}_{3} \mathrm{Si}_{2}\right)$. The impact of the characterization results is derived from the novelty of the process, where novel data on the laser synthesis of selected surrogate materials was acquired. The microstructural data acquired also assisted in predicting microstructural properties for future experiments with uranium-bearing compounds.

\section{Research Objectives}

The proposed research envisages to deliver novel microstructural data on the laser synthesis of surrogate silicide fuel. Hence, the following Objectives will provide the different research approaches employed to acquire novel results to develop the AMAFT technique. 


\section{Objective 1 - A Thermodynamic Analysis - Understanding Surrogate Fuel Formation}

Direct work with radioactive materials generally requires consideration of additional safety features. Hence, non-radioactive materials with properties comparable to those of uranium were advantageous to perform research experiments. From a safety standpoint, the use of surrogates provides a reduced risk of radiation exposure; from an economic perspective, it supports cost reduction for extensive experimental work. A better understanding of the kinetics in the formation of $\mathrm{U}_{3} \mathrm{Si}_{2}$ was developed by using the selected surrogates. One of the key operational challenges for surrogate experimental work is to establish safe work practices and demonstrate the experimental procedure prior to experimentation with uranium compounds.

The down selection of the surrogate materials was based on the crystallographic structure of the compounds, all preserving a tetragonal crystal structure $\left(\mathrm{U}_{3} \mathrm{Si}_{2}, \mathrm{Ce}_{3} \mathrm{Si}_{2}, \mathrm{Zr}_{3} \mathrm{Si}_{2}\right.$, and $\left.\mathrm{Hf}_{3} \mathrm{Si}_{2}\right)$ along with the similarities encountered on their respective phase diagrams. A survey of thermodynamic and mechanical properties of different compounds was conducted to examine the different characteristics and the suitability of the materials to undergo a laser synthesis. Some of the relevant properties evaluated were the crystallographic structure to ensure similar behavior of the surrogate compound to that of $\mathrm{U}_{3} \mathrm{Si}_{2}$. Also, the melting points of the down selected compounds were evaluated to adjust the laser parameters for optimum synthesis. Additionally, phase diagrams of the different surrogate materials were analyzed to establish a thorough understanding of the specific stoichiometry and temperatures required to form the desired compound (See Chapter 4).

Thermodynamic simulations with the ThermoCalc software package were performed to assist with experiment planning. With an extensive thermodynamic database, ThermoCalc can predict the formation of precipitate phases along with reactions between a material and its containment. ThermoCalc is a software package developed for computational materials 
engineering. It is widely used in applied research in applications for designing new alloys and optimizing processing conditions. Figure 1-3 displays the surrogate thermodynamic analysis scheme.

\section{Objective 2 - Effects of Laser Interaction on $\mathrm{U}_{3} \mathrm{Si}_{2}$ Surrogates - Characterization}

Characterization was performed on the surrogate samples synthesized with a laser beam source to evaluate the morphology and phases present (See Chapter 5). Understanding the interaction between the surrogate material and the laser source is crucial to develop suitable surrogates and implement the additive manufacturing process. The surrogate samples prepared by AMAFT were evaluated with different characterization techniques including electron microscopy (specifically SEM and EDS) and XRD.

Analyses on the laser-material interaction were performed through characterization on the end product to improve the material synthesis. Electron microscopy (specifically SEM and EDS) allowed the evaluation of the morphology and the defects produced by the laser ablation. XRD allowed the research team to determine the different secondary phases present on the compound. The characterization results provided novel data on $\mathrm{U}_{3} \mathrm{Si}_{2}$ surrogates undergoing a laser synthesis. The collected data was crucial to incorporate modifications in order to explore and ultimately develop the fabrication process. Figure 1-4 displays the work scope for evaluating the samples' suitability after the laser-material interaction.

\section{Objective 3 - A Prediction of the Formation of $\mathrm{U}_{3} \mathrm{Si}_{2}$ based on Surrogate Characterization Results}

Different characterization techniques including SEM, TEM, XRD, EDS, and SAD were performed on $\mathrm{U}_{3} \mathrm{Si}_{2}$ fuel samples manufactured through a powder metallurgical approach (conventional sintering). A microstructural comparison of the additive manufactured surrogates and the $\mathrm{U}_{3} \mathrm{Si}_{2}$ fuel samples was generated (See Chapter 6). The comparison results provided 
novel data that assisted in the prediction of $\mathrm{U}_{3} \mathrm{Si}_{2}$ microstructure undergoing laser synthesis.

Figure 1-5 shows the envisaged characterization outline leading to microstructural comparisons. These characterization results have increased the understanding of the formation of surrogate silicide fuel prior to utilizing uranium-bearing compounds as part of development of this novel additive manufacturing methodology.

\section{INL Long Term Goal: Development of the AMAFT Process}

INL has envisaged to develop the proof of concept of fabricating nuclear fuel through an integrated additive manufacturing methodology known as the AMAFT process. During Phase A, uranium surrogates will be undergo a laser synthesis and characterization results will assist to understand the interaction between surrogate material and the laser source. The data acquired will be crucial to develop suitable surrogates and incorporate modifications to develop the process. Phase A is composed of the following sub-phases:

- $\quad$ Phase 0: Verify the laser intensity and test the experimental set-up.

- $\quad$ Phase 1: Laser synthesis and characterization of surrogates by means of chemical reaction: $\mathrm{M}=$ metal; $3 \mathrm{M}+2 \mathrm{Si} \rightarrow \mathrm{M}_{3} \mathrm{Si}_{2}$.

- $\quad$ Phase 2: Direct formation of surrogate fuel $\mathrm{M}_{3} \mathrm{Si}_{2}$ from $\mathrm{UF}_{4}$ by means of a laser synthesis.

The current doctoral dissertation is solely focused on Phase 0 and Phase 1 within Phase

A. Phase B will include the laser synthesis of silicide fuel employing uranium compounds and Phase $\mathrm{C}$ will incorporate laser modifications into an integrated process to fabricate nuclear fuel in a commercial scale. The AMAFT experimental scheme is displayed on Figure 1-6.

This dissertation will provide novel characterization results on $\mathrm{U}_{3} \mathrm{Si}_{2}$ surrogates to assist in the prediction of microstructural properties for future experiments with uranium. The current research work intends to prove the concept of forming surrogate silicide fuel $\left(\mathrm{Zr}_{3} \mathrm{Si}_{2}\right.$ and $\left.\mathrm{Hf}_{3} \mathrm{Si}_{2}\right)$ employing a laser energy source. This work will support the development of the AMAFT process to fabricate accident tolerant fuel. Upon completion, the AMAFT process envisages to 
shorten the fabrication of $\mathrm{U}_{3} \mathrm{Si}_{2}$ fuel, which possess enhanced thermal properties, higher atomic density, and improved irradiation stability when compared to $\mathrm{UO}_{2}$. Ultimately, this accident tolerant fuel candidate will provide clean and reliable energy to benefit human kind. 


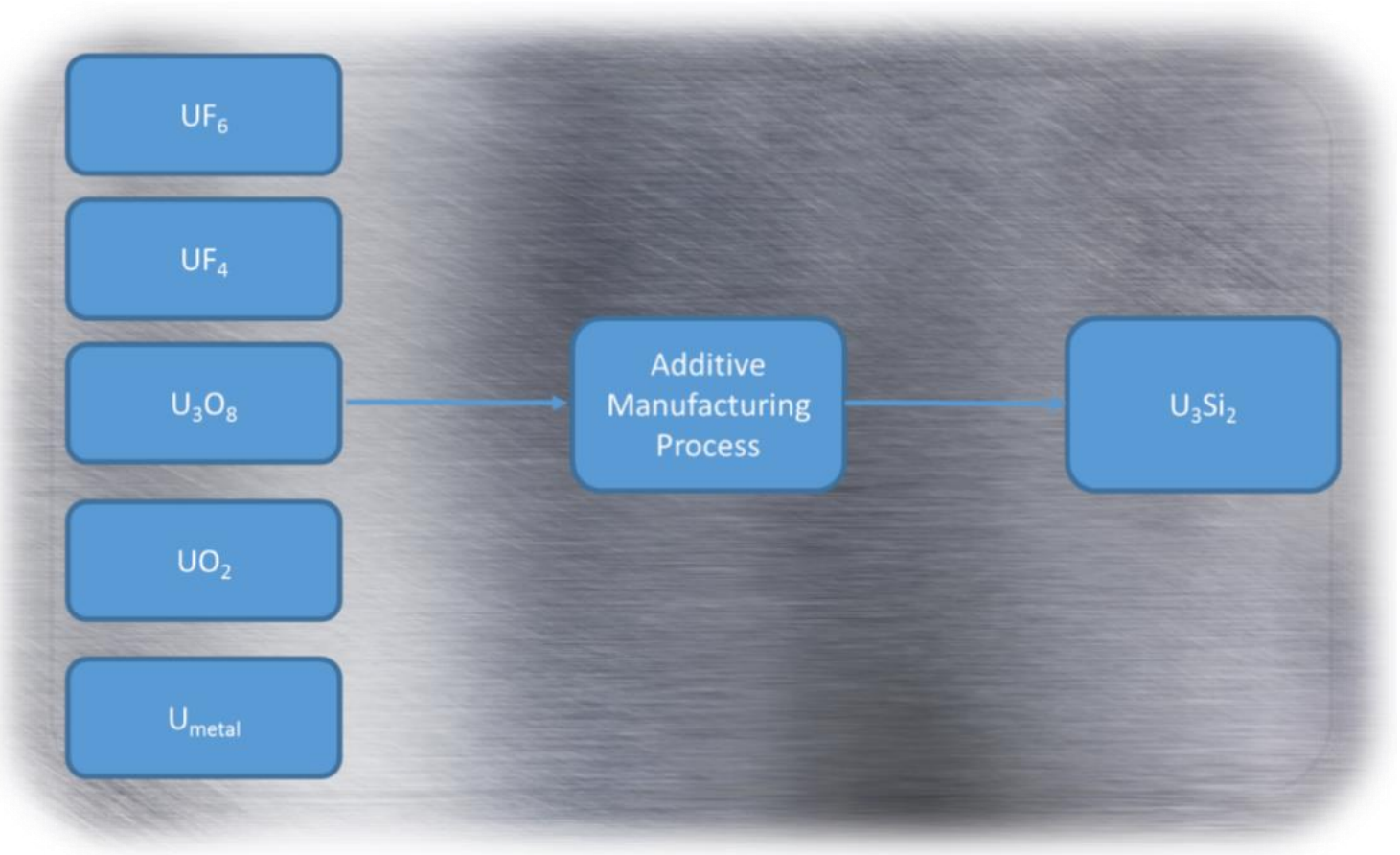

Figure 1-1. Potential compounds of the fuel cycle that will be utilized to produce $\mathrm{U}_{3} \mathrm{Si}_{2}$.

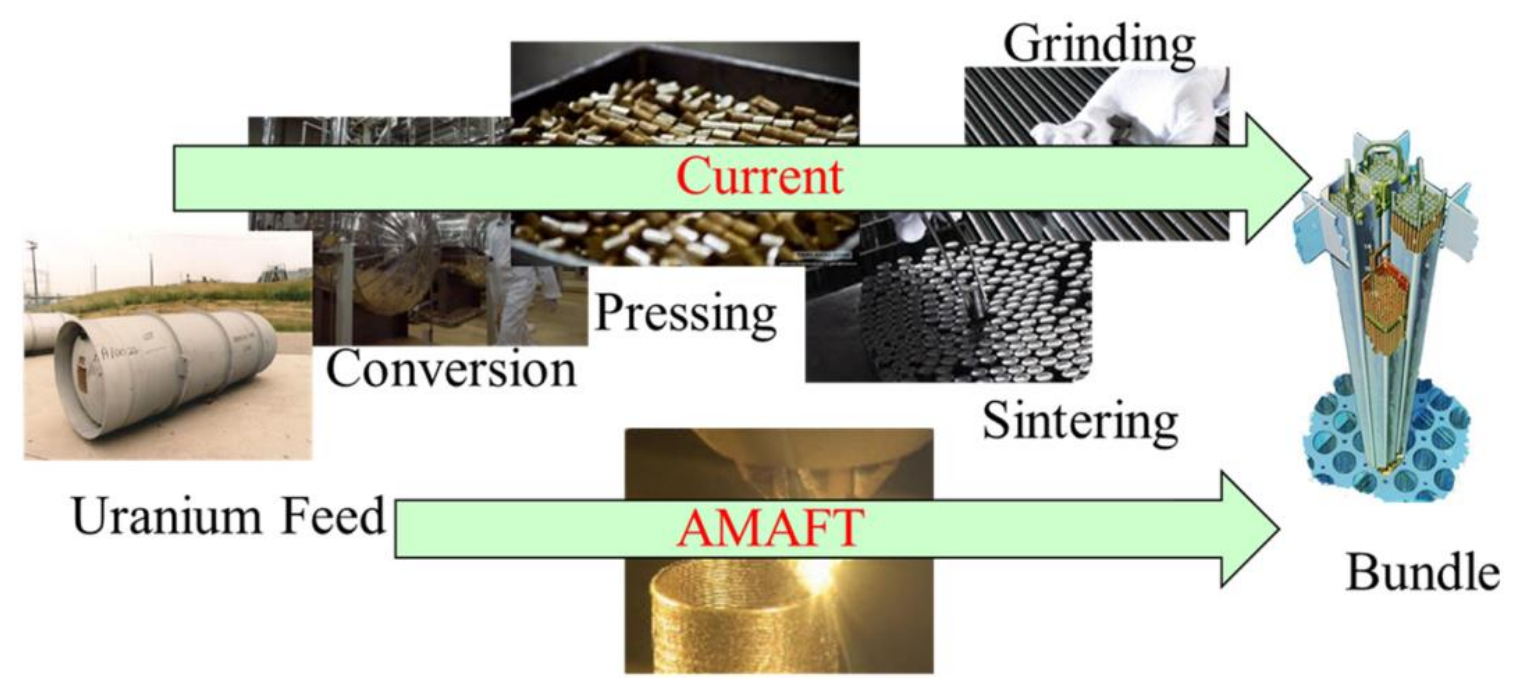

Figure1-2. Optimization scheme of the novel additive manufacturing process to produce $\mathrm{U}_{3} \mathrm{Si}_{2}$. 


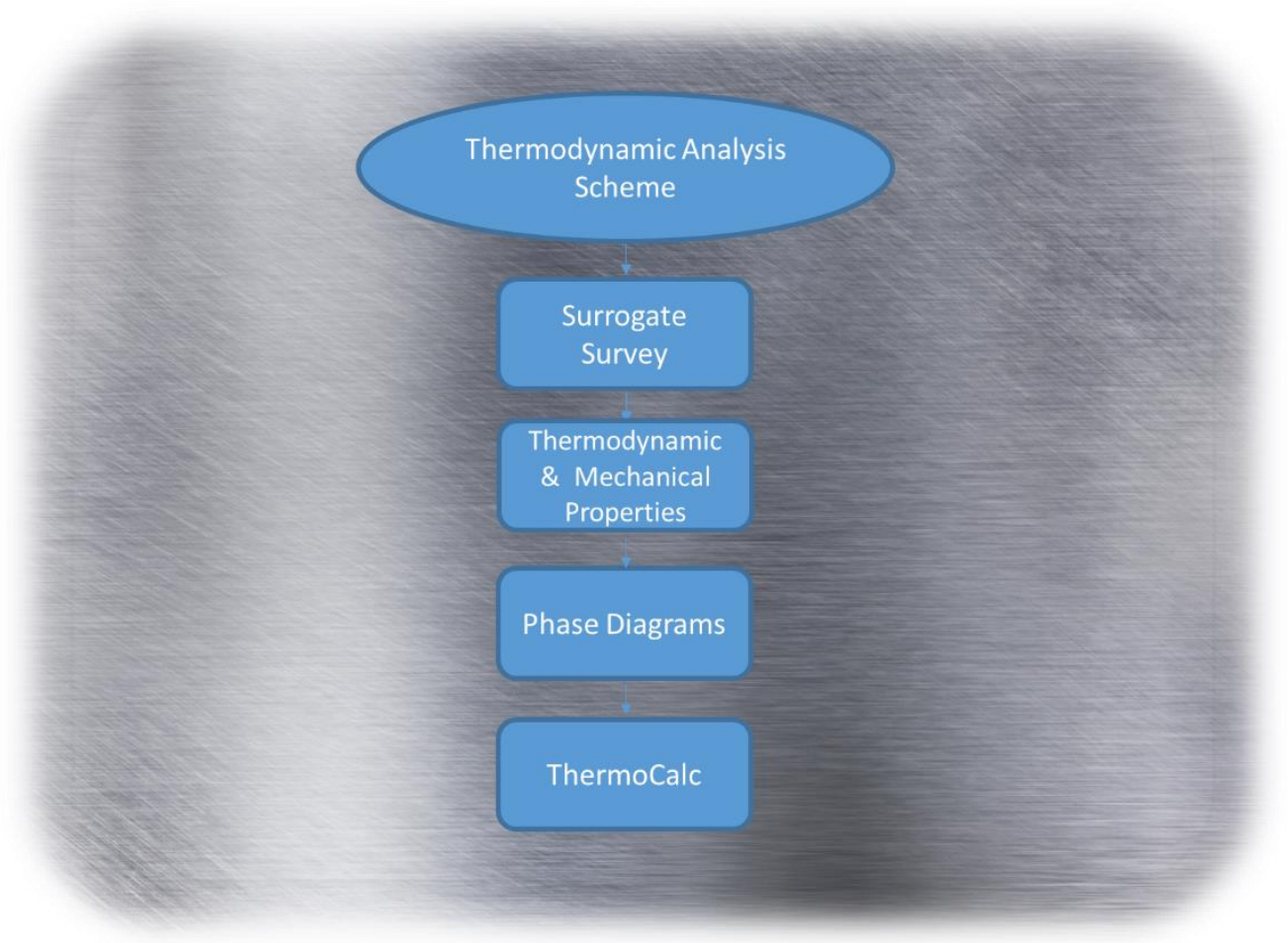

Figure 1-3. Thermodynamic analysis scheme.

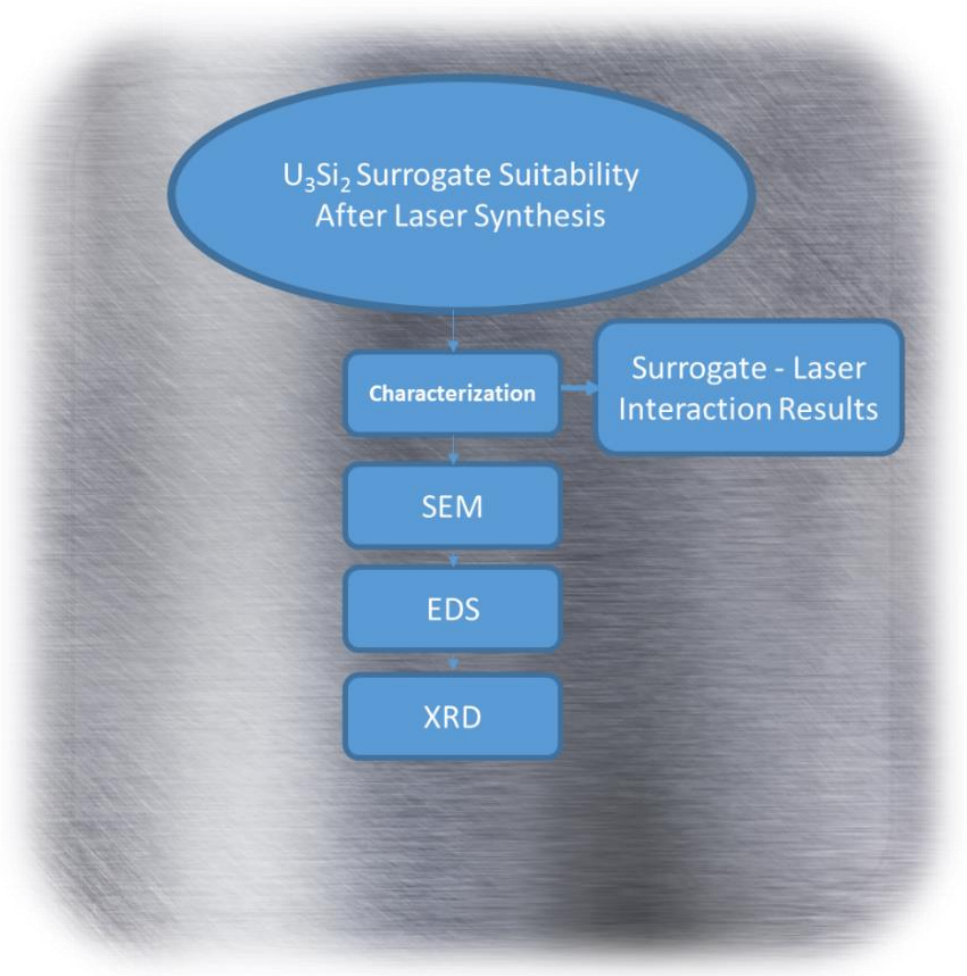

Figure 1-4. Laser-material interaction analysis scheme. 


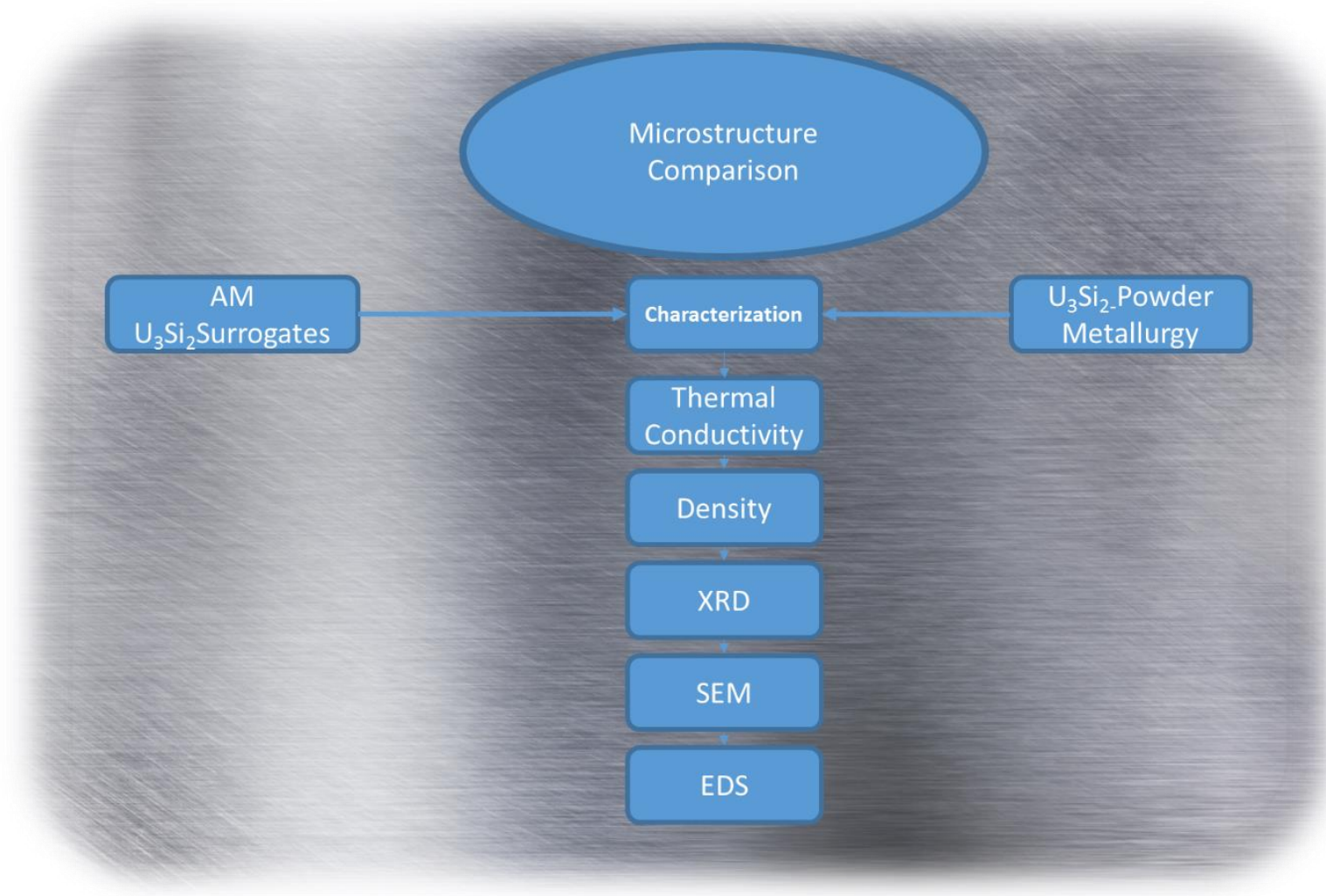

Figure 1-5. Characterization outline comparison of surrogate microstructure.

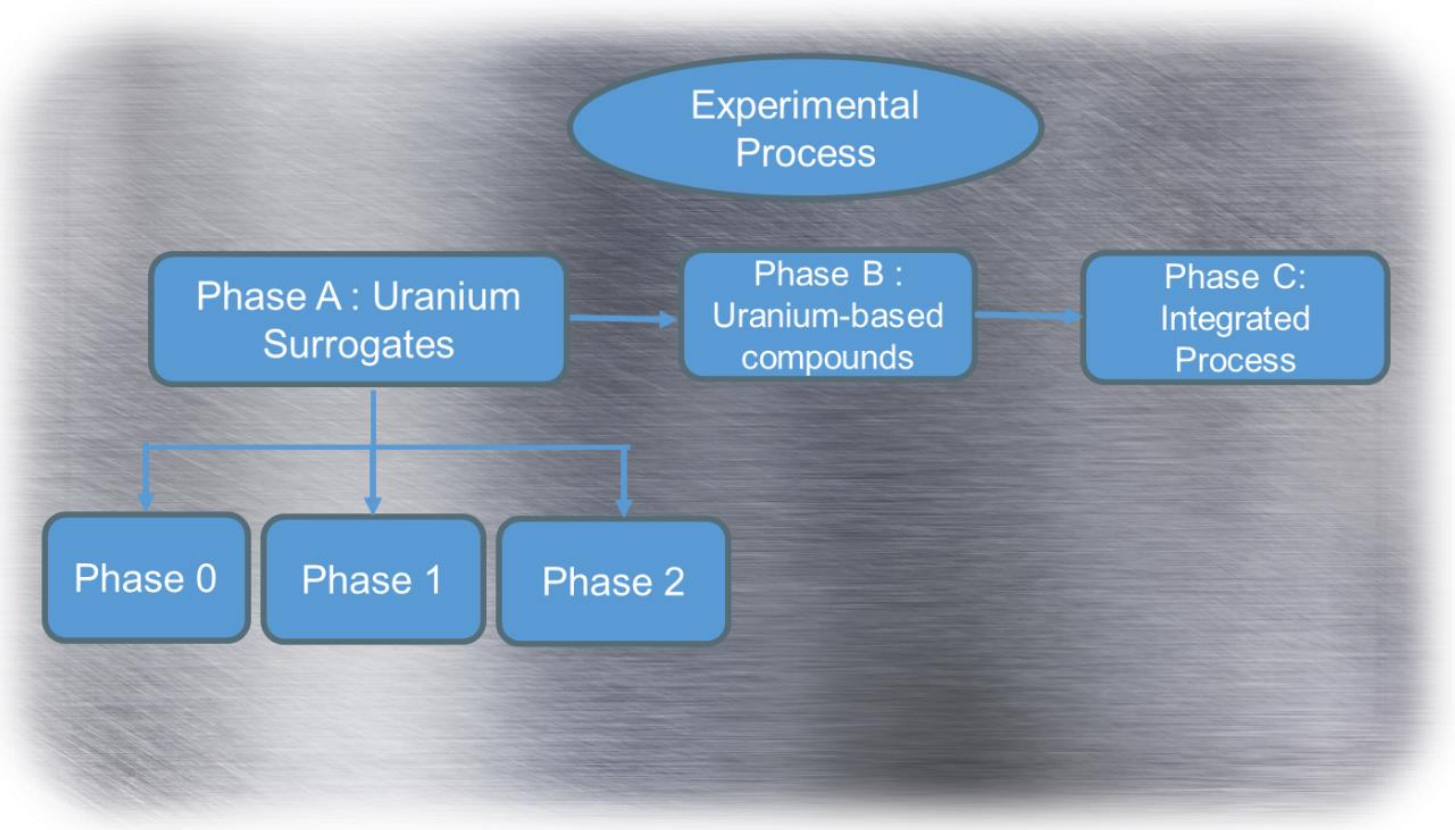

Figure 1-6. Experimental process to develop AMAFT. 


\section{CHAPTER 2 \\ METHODOLOGY}

This section describes the different characterization techniques and the specific equipment employed during the evaluation of the fabricated surrogate and $\mathrm{U}_{3} \mathrm{Si}_{2}$ fuel samples. All the characterization for this research work was performed at the Center for Advanced Energy Studies (CAES) located on the INL campus.

\section{Electron Microscopy}

\section{Scanning Electron Microscopy}

A scanning electron microscope employs a focused beam of electrons to produce a wide variety of signals at the surface of the evaluated target material. The signals originated from the electron-target interactions reveal data about the sample, including morphology, chemical composition, crystalline structure, and grain orientation [2].

The electron energy is dissipated in signals produced by the electron interactions when they are decelerated at the surface of the target sample. The signal includes secondary electrons, back scattered electrons, diffracted backscattered electrons, photons, visible light, and heat [3]. The secondary electrons and back-scattered electrons are employed for imaging samples. The secondary electrons can show morphology and topography of the target, and back-scattered electrons are useful for analyzing contrast in composition in multiphase samples. SEM analysis is considered a non-destructive technique, allowing the researcher to repeatedly evaluate samples without incurring any volume loss or alteration [3]. For this research work, a JEOL scanning electron microscope (model JSM-6610-LV) coupled with energy dispersive X-ray spectroscopy (EDS) was employed for imaging and chemical analyses. The apparatus is displayed in Figure 2-1(a). 


\section{Transmission Electron Microscopy}

A transmission electron microscope operates with a high energy beam of electrons traveling across very thin samples that are on the order of microns. The interactions among the incoming electrons and the target sample's atoms are analyzed to generate an image to observe microstructural features and defects. In addition, chemical analyses can also be performed as the majority of TEM's are equipped with an energy dispersive spectroscopy (EDS) system.

A TEM functions under a principle similar to that of light microscopes. The TEM employs the wavelength of the incoming electrons which is shorter than that of visible light. Thus, the resolution acquired under TEM imaging is very high compared to that of light microscopes, allowing the user to observe very fine details from the microstructure and sometimes small individual atoms [4].

A beam of electrons is focused into a smaller, thinner, and coherent beam by means of a condenser lens. The refined beam then strikes the target.Only a fraction of the beam is transmitted, depending on the electron transparency and thickness of the material. This transmitted beam fraction is projected onto a phosphorous screen. Objective apertures are employed to enhance contrast by blocking out high-angle diffracted electrons. The image then passes through a column with projector lenses. Finally, the image strikes a phosphor screen and light is generated, creating a high-resolution image [4].

For this research work a FEI Tecnai F30 microscope was employed and was operated at $300 \mathrm{Kv}$. Various STEM images were obtained using a camera focal length of $80 \mathrm{~mm}$. Multiple chemical analyses on TEM samples were carried out using the EDAX ${ }^{\mathrm{TM}}$ EDS system. Gatan Digital micrograph and TIA ${ }^{\mathrm{TM}}$ (TEM imaging and analysis) software were used for postprocessing the TEM data. Figure 2-1 (b) displays the TEM apparatus employed for this research project. 


\section{Focused Ion Beam}

Similar to an SEM, a focused ion beam (FIB) employs ions rather than electrons. The FIB can directly modify the target sample's surface by means of a sputtering process. Such a milling process can be controlled with nanometer-level precision. By controlling the energy and intensity of the ion beam the user can perform precise nano-machining and also removal of material. Also, ion-beam-assisted chemical vapor deposition can be employed to deposit material with a high precision, similar to FIB milling. A FIB can also be coupled with SEM on a dual system. In this case, the electron and ion beam intersect near the same sample surface, allowing immediate high-resolution SEM imaging of the FIB milled surface. The coupled technique was employed in this research work to produce FIB lamellae from uranium compounds for TEM analyses [5]. The FIB model employed for this research work was a FEI Quanta 3D FEG equipped with EDS, EBSD, and omniprobe. Figure 2-1 (c) displays the FIB apparatus employed in this research project.

\section{X-Ray Diffraction}

X-ray diffraction (XRD) spectroscopy analyzes both the wave and particle properties of $\mathrm{X}$-rays to acquire information about the structure of the evaluated crystalline material. The main use of this technique is to identify and characterize compounds based on their specific diffraction pattern. When an incident beam of X-rays interacts with a target material, X-rays scatter from the material's atoms, creating a unique diffraction pattern. The scattered X-rays undergo destructive and constructive interference upon impact. The direction of the scattered X-rays strictly depends on the size and shape of the unit cell of the target material. The intensity of the diffracted waves is affected by the atomic arrangement of the crystal structure. The diffraction pattern will be unique for every atom, allowing to identification the composition of the target material. 
XRD has been widely used to study the specific composition of the microstructure in materials of interest. For this research work, a Rigaku Smartlab X-ray Diffractometer was employed to evaluate the crystallographic properties of the produced samples. Figure 2-1 (d) displays the Rigaku Smartlab X-ray Diffractometer employed for this work. 

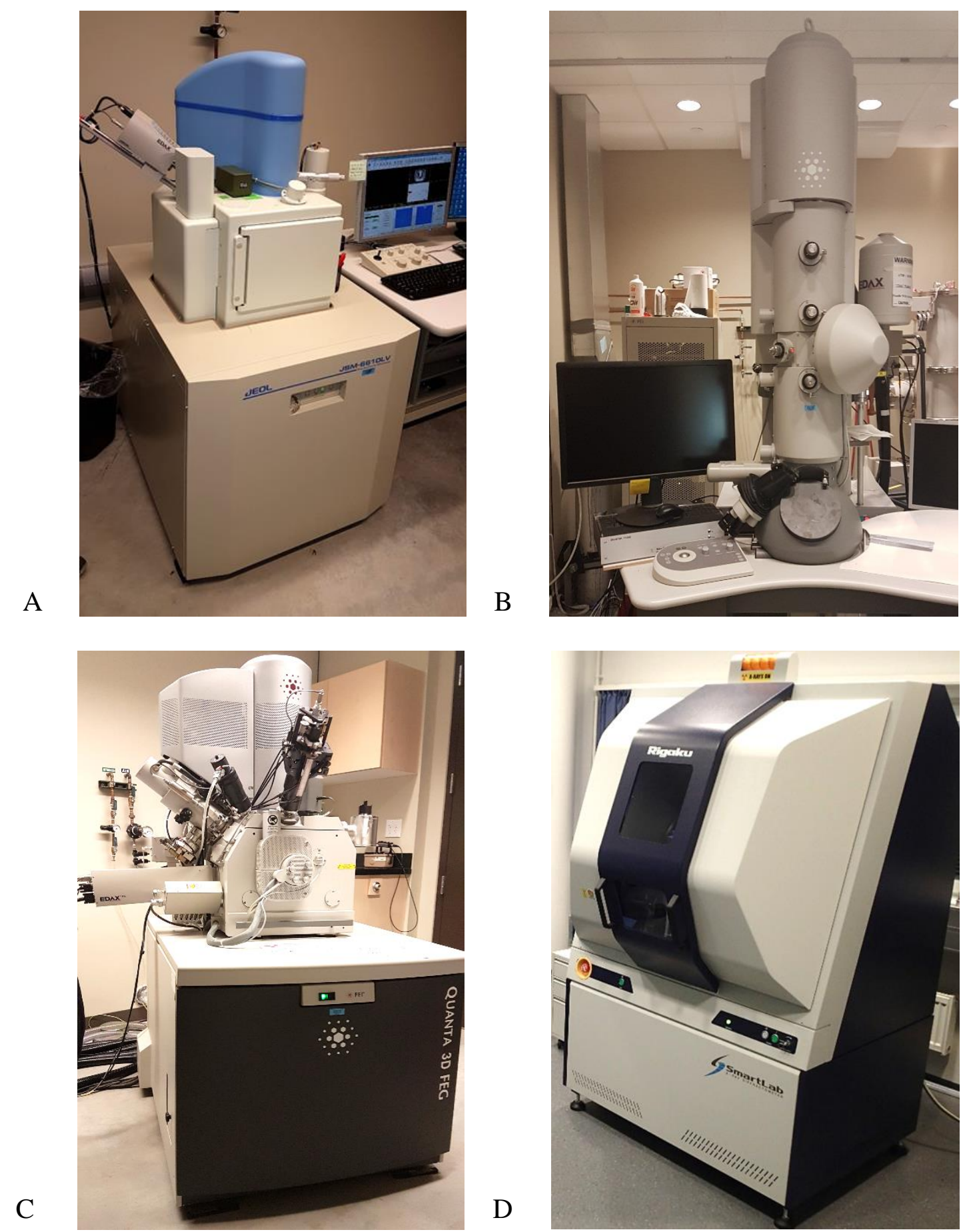

Figure 2-1. Characterization equipment employed in this research. The equipment was operated at CAES at the INL campus. A) A JEOL scanning electron microscope model JSM6610-LV, equipped with energy dispersive X-ray spectroscopy. B) A FEI Tecnai F30 transmission electron microscope. C) A FIB model FEI Quanta 3D FEG equipped with EDS, EBSD, and omniprobe. D) A Rigaku Smartlab X-ray diffractometer. 


\section{CHAPTER 3 \\ LITERATURE SURVEY \\ $\mathrm{U}_{3} \mathrm{Si}_{2}$ Surrogates}

Uranium surrogate materials have been widely employed for different purposes, including creating a radiation-free work environment and reducing economic costs. A nonradioactive surrogate material with comparable properties to those of uranium is cerium. Cerium can form compounds with complex magnetic behavior [6]. $\mathrm{CeO}_{2}$ displays a cubic fluorite structure from room temperature up to its melting point of $2,400^{\circ} \mathrm{C}$ and is iso-structural with both $\mathrm{UO}_{2}$ and $\mathrm{PuO}_{2}$. In addition, $\mathrm{CeO}_{2}$ has a low solubility and high radiation stability, exhibiting a behavior under an irradiation environment similar to that of $\mathrm{UO}_{2}$ and $\mathrm{PuO}_{2}$ [7]. Due to these similarities, $\mathrm{CeO}_{2}$ has been employed as surrogate in studies of accident-tolerant fuels. $\mathrm{Ce}_{3} \mathrm{Si}_{2}$ is isostructural with $\mathrm{U}_{3} \mathrm{Si}_{2}$ and has been previously used as a $\mathrm{U}_{3} \mathrm{Si}_{2}$ surrogate in studies of processing techniques, oxidation, corrosion, and transport properties [6]. In addition, the $\mathrm{Ce}-\mathrm{Si}$ and U-Si phase diagrams have numerous similarities as depicted on Figure 4-9 and Figure 4-12, respectively.

Studies show that a wide range of cerium silicides, including $\mathrm{Ce}_{3} \mathrm{Si}_{2}$, were produced by high-energy ball milling (HEBM), which is a self-enclosed processing technique that can limit oxygen contamination along with silicon and cerium loses. The synthesis of silicides by means of HEBM was first reported nearly 30 years ago [6]. Alanko et al. showed the feasibility of producing $\mathrm{Ce}_{3} \mathrm{Si}_{2}$ using a mechano-chemically-induced self-propagating reaction (MSR). Silicon and cerium powders were deposited inside a zirconia milling vessel and were milled at $500 \mathrm{rpm}$ for 6 hours [6]. EDS analyses confirmed minimum contamination of less than $1 \% \mathrm{Zr}$ and $\mathrm{Fe}$ from the milling media and vessel. Figure 3-1 shows the SEM micrographs before and after MSR. 
Additional densification procedures followed the MSR synthesis. Spark plasma sintering (SPS) was performed on the milled powders. The samples were loaded into graphite dies under a nitrogen atmosphere with oxygen levels below 200 ppm. The dies had a 21-mm bore and 15mm-thick wall. They were lined with graphite foil. The sintering occurred at a temperature of $1,000^{\circ} \mathrm{C}$ with hold times of 5 minutes. An axial pressure of $5 \mathrm{KN}$ was held throughout the temperature ramp until cooldown [6]. Figure 3-2 shows the sintering temperature, pressure, and Z-axis displacement as a function of time. Figure 3-3 displays an SEM picture of a fracture surface demonstrating the high density achieved ( $95 \%$ TD) and a fine grain structure of the sintered material [6].

The feasibility of employing zirconium as a uranium surrogate has been analyzed. Zirconium has comparable chemical and crystallographic properties to those of uranium. There is not enough evidence of research work on $\mathrm{Zr}_{3} \mathrm{Si}_{2}$ for fuel manufacturing; however, studies have shown that $\mathrm{ZrO}_{2}$ has been successfully employed as a surrogate for uranium fuel in TRISOcoated fuel particles. Surrogate TRISO fuel was produced for irradiation testing at the High Flux Isotope Reactor (HFIR) at Oakridge National Laboratory [8]. Other studies on new methods for High Temperature Reactor (HTR) fuel waste management also simulated the presence of fuel in TRISO particles by using $\mathrm{ZrO}_{2}$ kernels [9]. The previous research proved the suitability in employing zirconium as a surrogate material for uranium.

\section{Optical Properties of Matter}

Laser irradiation-induced effects on materials involve different aspects, including optical, electromagnetic, thermodynamic, and mechanical changes in material properties, making the laser-matter interaction an interdisciplinary and complex subject [10]. The study of laser-matter interaction is an appropriate method to study the material properties and to understand the intrinsic microstructure of materials. The optical properties include the index of refraction $n$, 
which is the ratio of the speed of light in a vacuum to its speed in the medium as light propagates through the material. Such a ratio shows how light slows down while traveling through a specific material. See Equation 3-1, where $c$ is the speed of light in a vacuum and $v$ is the velocity of light in the medium.

$$
n=\frac{\mathrm{c}}{\mathrm{v}}
$$

For absorbing media, the intensity of the electromagnetic wave propagating into the material tends to exponentially decrease following the Lambert-Beer expression in Equation 3-2, where the absorption coefficient $\alpha$ is the inverse of the absorption length in the material. $I$ is the intensity as a function of absorption length $z, I_{o}$ is the initial intensity of the beam, $k$ is the extinction coefficient and $\lambda$ represents the wavelength [11].

$$
I(z)=I_{o} e^{-\alpha \cdot z} \quad \text { where } \quad \alpha=\frac{4 \pi k}{\lambda}
$$

In the aforementioned expression, radiation absorption is dependent on the wavelength $\lambda$ along with the thickness $z$ and nature of the medium. The absorption coefficient can be described as the reciprocal of the depth of penetration of radiation into the target solid. For normal incidence of light, the reflectivity of a material can be defined as the incident to reflected intensity ratio, and the expression is given in Equation 3-3. In this equation, $n$ and $k$ represent the index of refraction and extinction coefficient, respectively [11].

$$
R=\frac{(n-1)^{2}+k^{2}}{(n+1)^{2}+k^{2}}
$$

\section{Laser Thermal Effects}

Materials exposed to laser irradiation will absorb the incident energy from the laser.

Consequently, the material will experience a raise in temperature generating material expansion and thermal stress. After the stress exceeds a certain value, the material can fracture and experience plastic deformations. The target material can absorb the incident laser energy, of 
which only a fraction will be converted into heat. A fluctuating temperature distribution will be displayed due to the uneven heat diffusion. Expansion and contraction can generate laserinduced thermal stress [10]. Figure 3-4 displays thermal fracture and melting of $\mathrm{SiO}_{2}$. The deposition of laser energy on the target material can result in thermal depolarization in crystals for doubling or tripling the laser's frequency. Second harmonic generation occurs when photons with the same frequency interact with a non-linear material and combine to generate new photons with twice the initial energy and, therefore, twice the frequency of the initial photons. Figure 3-5 displays the process of second harmonic generation [10].

When a material is irradiated with a laser source, the energy will initially be transformed into electric excitation energy; then it transfers to the material lattices through collisions between electrons [10]. When large amounts of energy are deposited in the atom, macroscopic changes occur, such as an increase in temperature, ionization, or gasification.

As the laser energy increases, the target material will absorb significant amounts of energy, and the deposited energy will cause the material to melt when exceeding the material's melting temperature. Melting, along with solidification, can affect the atomic structure of materials and produce transformations between crystalline and amorphous states. Equations 3-4 and 3-5 [13] describe the temperature distribution in bulk materials. In these equations, ierfc is the complementary error function, $I$ is the intensity of the laser beam in $\mathrm{W} / \mathrm{m}^{2}, \alpha$ is the optical absorption coefficient, $R$ is the reflectivity, $D$ is the thermal diffusivity, $k$ is the thermal conductivity, $t$ is time, $t_{p}$ is laser pulse time, and $T$ is the temperature yield at a depth $z$.

$$
\begin{gathered}
T(z, t)=\frac{2 \alpha I(1-R)}{K} \sqrt{D t} \operatorname{ierfc}\left(\frac{z}{2 \sqrt{D t}}\right) \\
\text { When }\left(t<t_{p}\right)
\end{gathered}
$$




$$
T(z, t)=\frac{2 \alpha I(1-R)}{K}\left((\sqrt{D t}) \operatorname{ierfc}\left(\frac{z}{2 \sqrt{D t}}\right)-\left(\sqrt{\left(t-t_{p}\right) D}\right) \operatorname{ierfc}\left(\frac{z}{2 \sqrt{\left(t-t_{p}\right) D}}\right)\right)
$$

Laser heating depends on different laser parameters, including laser wavelength, power density, and pulse duration. The effects can be categorized as thermal or mechanical. Thermal effects involve melting, vaporization, boiling, and phase explosion. Mechanical effects include deformation and resultant stress in the material. Only the amount of energy absorbed by the material will contribute to heating and melting. Figure 3-6 displays laser-material interaction with a polycrystalline silicon.

The plasma absorbs the incident waves and exchanges energy with the lattice. Table 3-1 displays both gains and losses influenced by plasma formation. At high intensities of the laser pulse $\left(>10^{6} \mathrm{~W} / \mathrm{cm}^{2}\right)$, evaporation and plasma effects become relevant. Vapor clouds begin to form with different and inhomogeneous indexes of refraction, producing a distortion on the incident waves. The distance from the material's surface to the vapor plasma is crucial for determining the different effects. When the plasma remains at the material's surface, its emission of blackbody radiation is efficiently absorbed by the material. For higher intensities, ionization of vapor occurs and the breakdown plasma propagates against the incident beam, shielding the material. At extremely high-pulse densities $\left(>10^{9} \mathrm{~W} / \mathrm{cm}^{2}\right)$ the plasma becomes transparent, the wave front interacts with the material, and the ablation pressure initiates a shockwave into the material, affecting both structural and optical properties of the material [11]. 


\section{$\mathrm{U}_{3} \mathrm{Si}_{2}$ as an Accident Tolerant Fuel}

One of the main advantages of $\mathrm{U}_{3} \mathrm{Si}_{2}$ is its high atomic density. A higher uranium density fuel like $\mathrm{U}_{3} \mathrm{Si}_{2}$ will allow the same number of fissile $\mathrm{U}^{235}$ atoms to be accommodated in a smaller volume of material. This property can lead to large savings in both enrichment and fuel manufacturing costs, which are crucial aspects to evaluate in the selection of an accident-tolerant fuel. The silicide fuel with $\mathrm{U}_{3} \mathrm{Si}_{2}$ phase has a density of $12.2 \mathrm{~g} / \mathrm{cm}^{3}$, achieving a $17 \%$ increase in uranium density over $\mathrm{UO}_{2}\left(\rho_{\mathrm{U}}=11.31 \mathrm{~g} / \mathrm{cm}^{3}\right.$ for $\mathrm{U}_{3} \mathrm{Si}_{2}$ compared with $\rho_{\mathrm{U}}=9.66 \mathrm{~g} / \mathrm{cm}^{3}$ for $\left.\mathrm{UO}_{2}\right)$ [14]. Table 3-2 shows the density of various fuel compounds, placing $\mathrm{U}_{3} \mathrm{Si}_{2}$ among the fuels with the highest density.

The thermal properties of the silicide fuel are vastly superior to those of $\mathrm{UO}_{2}$. The comparatively improved thermal conductivity of $\mathrm{U}_{3} \mathrm{Si}_{2}$ can decrease the thermal gradients and generate lower centerline temperatures during fuel performance [14]. Studies have successfully demonstrated that, at room temperature, the thermal conductivity of $\mathrm{UO}_{2}$ is just a fraction compared to that of silicide fuel [15]. Also, from a safety perspective, improvements in thermal conductivity can potentially slow down the rise rate of the core temperature during accident conditions. Additionally, the $\mathrm{U}_{3} \mathrm{Si}_{2}$ irradiation stability of the fuel is promising, with fission gases forming small uniform bubbles with minimal coalescence, limiting fuel swelling, which brings stability during high burnups [16]. Some of the key properties of $\mathrm{U}_{3} \mathrm{Si}_{2}$ are shown on Table 3-3 [14].

\section{Fabrication Methods}

The actual commercial production of $\mathrm{U}_{3} \mathrm{Si}_{2}$ involves a vacuum induction melting followed by annealing. Studies show that a phase pure compound can be produced by powder metallurgy [14][17]. In addition, production of uranium silicide by HEBM in a planetary ball mill proved that extended milling times between 4 and 12 hours slowly allowed the formation of 
$\mathrm{U}_{3} \mathrm{Si}_{2}$ while allowing $\mathrm{USi}_{3}$ to react with the remaining uranium. Such results proved that mechano-chemical synthesis is a feasible method to produce $\mathrm{U}_{3} \mathrm{Si}_{2}$ composites [18]. Another common method is centrifugal atomization, in which the molten metal compounds are fed through a nozzle into a spinning disk. The molten alloy droplets are spread by the disk by centrifugal force. Such a process allows a quick microstructure solidification and a narrow particle distribution [19].

\section{Powder Metallurgy Fabrication}

Samples of high-density uranium silicide have been fabricated by powder metallurgy techniques [14]. This process for producing $\mathrm{U}_{3} \mathrm{Si}_{2}$ fuel has the potential to be industrially scalable. It involves handling uranium powder metal, which is highly pyrophoric and should be kept in an inert gas atmosphere within a glovebox. During the fuel fabrication process (illustrated in Figure 3-7) the uranium powder was produced by hydride/dehydride process and the necessary silicon was added as a powder. Then, the compound was pressed into a compact, which was agglomerated in a furnace at $1,450^{\circ} \mathrm{C}$ and arc melted to produce a homogenous alloy. This process produced a pure phase at $97 \% \mathrm{U}_{3} \mathrm{Si}_{2}$ [14]. After conducting XRD examinations on the samples, concentrations of 0.5 and $3.3 \mathrm{wt} \% \mathrm{U}_{3} \mathrm{Si}$ were revealed in the fabricated uranium silicide and no $\mathrm{UO}_{2}$ phase was identified after completing the arc melting process. This phase purity of $\mathrm{U}_{3} \mathrm{Si}_{2}$ is superior to the conventional uranium silicide produced for research and test reactors [14]. Figure 3-8 shows an SEM image of the of $\mathrm{U}_{3} \mathrm{Si}_{2}$ purity phase, in which the dark grey areas are the $\mathrm{U}_{3} \mathrm{Si}_{2}$, the lighter areas the $\mathrm{U}_{3} \mathrm{Si}$, and the dark sites are porosities. After extracting the uranium silicide from the arc melter, the samples were reduced to a fine powder before being pressed between 124 and $156 \mathrm{MPa}$ and compacted into a green pellet. The sintering

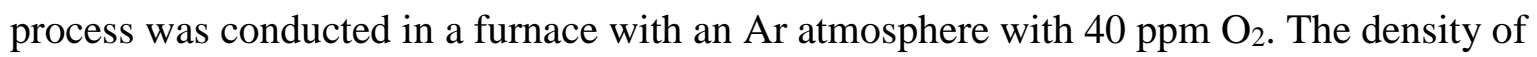
sintered pellets highly relies on the grain size, green density, maximum sintering temperature, 
and time held at the maximum sintering temperature. The sintering times and temperatures employed ranged from 2 to 8 hours and 1,200 to $1,500^{\circ} \mathrm{C}$, respectively. The highest densities measured from the samples of this manufacturing process ranged from 96.1 to $97.8 \%$ theoretical density. Examination of the microstructure via SEM was performed on the sintered samples. The SEM analysis revealed the absence of $\mathrm{U}_{3} \mathrm{Si}$ in the fabricated composites. Figure 3-9 displays the microstructure on a sintered sample of $\mathrm{U}_{3} \mathrm{Si}_{2}$ fuel. In addition, XRD was also performed on the final samples and examination confirmed that the primary phase in the sintered pellets was of $\mathrm{U}_{3} \mathrm{Si}_{2}$ in which no uranium oxide phases were found and a $0.5 \% \mathrm{U}_{3} \mathrm{Si}$ concentration was observed [14]. This process proved the optimization of pressing and sintering parameters to fabricate samples with a high-density and a pure phase.

\section{Centrifugal Atomization}

Other powder preparation methods have been developed to improve the properties of the fuel. The centrifugal atomization process consists of having the molten metal heated to approximately $200^{\circ} \mathrm{C}$ above its melting point, then run through a nozzle leading to a rapidly rotating graphite disk on a vertical axis [20]. Then the liquid alloy drops are spread from the disk by means of centrifugal force and cooled at an argon atmosphere. In addition, the powder size can be controlled by adjusting operation parameters, such as the feeding rate of the melt and revolution speeds of the disk [19]. In one experiment the approximate feeding rate, along with the revolutions of the disk, were estimated to be 8,000 g/min and 32,000 rpm, respectively [19]. Figure 3-10 provides a graphic description of the apparatus employed.

As the fuel particle size decreases, a greater cooling rate is achieved, leading to finer $\mathrm{U}_{3} \mathrm{Si}_{2}$ precipitates. This occurs due to homogeneous nucleation and massive solidification. Such observation suggested that a rapid cooling rate increases with a finer powder due to the increase in surface area, allowing reduced solidification times. $\mathrm{U}_{3} \mathrm{Si}_{2}$ particles generally hold a structure 
with fine grains below $5 \mu \mathrm{m}$ in size [19]. Grains are finely dispersed and silicon-rich precipitates can be found at grain boundaries. The fine structure is attributed to the high cooling rate before solidification. Figure 3-11 displays the microstructure of the atomized uranium silicide powders. From SEM analyses it was concluded that most particles had nearly perfect spherically shapes with smooth surfaces and a few precipitates attached.

Some of the advantages of the atomization process are that powders have a rapidly solidified microstructure, a relatively narrow particle size distribution, and a uniformly spherical shape [19][20]. Studies have shown that fuel samples from atomized powders displayed a smaller volume increase after annealing compared to samples from comminuted powder, irrespective of the temperature gradient and heat treatment. It has been assumed that atomized fuel samples contain lower as-fabricated porosity. This provides more void space for gas release and a smaller restraint for swelling [20]. Figure 3-11 shows a porosity comparison between the atomized and the comminuted processes. An 80-um particle diameter was achieved at a rotating speed of $30,000 \mathrm{rpm}$ with a feeding rate of $2,500 \mathrm{~g} / \mathrm{min}$ [19]. Using this innovative method, the particle size distribution of the atomized powders becomes finer and more uniform, and enhances the structural properties of the fuel. This is arguably a simplified method that can enable mass production, improve yield, and provide a purer product. The process has shown improved thermal and mechanical properties in the fabricated fuels [19][20][21].

\section{High Energy Ball Milling}

HEBM has been employed to fabricate alloys and other nuclear materials, such as uranium nitride and nuclear surrogates. The advantages of this method include the ease of formation of in situ composites and low processing temperatures [18]. The difficulties encountered in this process include the impurity content generated by the milling equipment. A planetary ball mill and a $250-\mathrm{mL}$ steel milling vessel were used for this procedure. The sample 
powders were placed inside a ceria-stabilized zirconia (CSZ) media, which was under an argon atmosphere and inside a glovebox due to the pyrophoric nature of the end product $\mathrm{U}_{3} \mathrm{Si}_{2}$ in powder form. The milling stage was conducted at $500 \mathrm{rpm}$ between 5 minutes and 24 hours. In this process the desired silicide phase $\mathrm{U}_{3} \mathrm{Si}_{2}$ was achieved in less than 12 hours of milling the sample. Figure 3-12 shows the microstructure observed at different times during the milling process. Additionally, the U-Si phase content and relevant parameters of the HEBM process are displayed in Table 3-4 [18].

Studies of the process revealed that $\mathrm{U}$ and $\mathrm{Si}$ rapidly react to form $\mathrm{U}+\mathrm{USi}_{3}$, which consequently produces $\mathrm{U}_{3} \mathrm{Si}_{2}$ after 3.5 to 12 hours of milling time. The milled powder adheres to the milling media, generating loses of approximately $10 \%$ of the total powder load. The phase formation occurs in the following way:

1. 5 minutes: The uranium powder deforms from $100-\mu \mathrm{m}$ spheres into $10-\mu \mathrm{m}$-thick sheets. $\mathrm{U}_{3} \mathrm{Si}$ forms along the contact areas between the highly strained uranium and the silicon.

2. 1 hour: Laminar structure $\mathrm{U}$ and $\mathrm{U}_{3} \mathrm{Si}$ sheets develop in irregularly shaped particles.

3. $\quad 3$ hours: $\mathrm{U}$ and $\mathrm{U}_{3} \mathrm{Si}$ particle shape changes.

4. 8 hours: Reaction to $\mathrm{U}_{3} \mathrm{Si}_{2}$ is almost complete with $10-50 \mu \mathrm{m}$ particles.

5. 12 hours: Fracturing/cold welding increases in pure $\mathrm{U}_{3} \mathrm{Si}_{2}$ [18].

The milling results displayed contamination in the form of $\mathrm{UO}_{2}$. The oxygen contamination can be attributed to oxide films on the starting materials or to the reduction of the milling media by any of the uranium phases developed along the process. Figure 3-13 shows the XRD analysis performed at different stages throughout the 12-hour milling process. Based on the XRD analysis, a fairly pure $\mathrm{U}_{3} \mathrm{Si}_{2}$ phase was achieved using this process. These results suggest that mechanochemical synthesis is a viable technique for production of $\mathrm{U}_{3} \mathrm{Si}_{2}$ fuel.

\section{$\mathrm{U}_{3} \mathrm{Si}_{2}$ Cladding Compatibility}

Accident-tolerant fuels have been proposed to enhance the safety performance of power reactors under different types of transients and severe accident conditions. The main features to 
consider are: a slower oxidation of cladding in steam to reduce hydrogen $\left(\mathrm{H}_{2}\right)$ generation, an improved capability of cladding to maintain the cool-ability of the core, and improved retention of fission products within the fuel. The promising cladding materials compatible with the $\mathrm{U}_{3} \mathrm{Si}_{2}$ system include $\mathrm{SiC}$ and $\mathrm{FeCrAl}$, which can potentially improve the accident-tolerant performance [14]. FeCrAl designs show outstanding high-temperature mechanical strength and oxidation resistance during accident conditions. SiC presents a desirable low neutron absorption cross section and a satisfactory irradiation performance under normal operating conditions. Drawbacks of $\mathrm{SiC}$ are manufacturing challenges and potential fuel-clad mechanical interactions [14]. Figure 3-14 displays the thermal conductivity of various cladding candidates from 300 to $3,300 \mathrm{~K}\left(\sim 27-3,027^{\circ} \mathrm{C}\right) . \mathrm{SiC}$ and $\mathrm{FeCrAl}$ clearly show an improved thermal conductivity in relation to the rest of the cladding candidates evaluated.

A research study included a safety analysis of a pressurized water reactor (PWR) fueled with accident-tolerant fuel under severe accident conditions. The study was performed using the PWR severe accident analysis code MIDAC [22]. A large break loss of coolant accident (LBLOCA), in which a break in the full-power level leads to a large loss of coolant in the reactor vessel, was simulated. As a consequence, the decay energy causes a drastic increase in the cladding temperature as shown in Figure 3-15. During the simulation, the geometric parameters were consistent with those of a conventional $\mathrm{UO}_{2}-\mathrm{Zr}$ PWR core. Also, the accident-tolerant fuel materials lose integrity when either the fuel or cladding temperature exceeds the corresponding melting point and is categorized as a failure criterion.

Other studies highly recommend the use of $\mathrm{SiC}$ as one of the main candidate materials for fuel cladding in lead-cooled fast reactor and very high-temperature reactor designs [22][23]. As a cladding material, $\mathrm{SiC}$ has great advantages over other materials, such as improved high- 
temperature corrosion properties leading to enhanced thermodynamic efficiency. SiC also has a greater high-temperature strength and a low-neutron absorption cross section. In addition, SiC composites have proven to have a stable irradiation behavior and are expected to retain their mechanical integrity at radiation levels above 50 dpa with temperatures close to $1,000^{\circ} \mathrm{C}$ [23].

The main concern about the use of $\mathrm{SiC}$ as an accident-tolerant fuel candidate is its brittle behavior. It can produce clad failure due to pellet-clad mechanical interaction. It is also susceptible to oxidation under specific conditions, and its vulnerability to stress corrosion cracking is still being investigated [23]. Cost and chemical stability with accident-tolerant fuel and coolant are the major concerns facing the development of $\mathrm{SiC}$ as a cladding material [23].

\section{Stoichiometric U3Si2 Phase}

The production of silicide fuels generally includes relative amounts of the various phases or distinguishable crystalline entities present in the U-Si alloy at different Si concentrations [1]. From previous studies, it may be considered essentially impossible to produce an alloy at the exact stoichiometry of the compound and of such atomistic homogeneity that only a pure phase is present. A minor, but finite, amount of the phase to the left or the right (phase diagram) of the compound of interest can be found in the microstructure [1]. Furthermore, to increase the challenge of producing a pure phase, the presence of impurities, which are inevitable, can allow the formation of other phases that may or may not be noticeable under microscopy, depending on the size and the magnification employed. The impurities found can be expected to appear in a solid state, sometimes within the crystalline lattices, in each of the phases present. A study on the chemical analysis performed on $\mathrm{U}_{3} \mathrm{Si}_{2}$ pellets fabricated by powder metallurgy shows the chemical variations within the samples. The results of the analysis for detectable elements are shown in Table 3-5 [16]. XRD was also performed in the study. Figure 3-16 displays the XRD peaks confirming that $\mathrm{U}_{3} \mathrm{Si}_{2}$ is the dominant phase present in the sample. The characteristic peaks 
of different $\mathrm{U}-\mathrm{Si}$ phases in the proximities of $\mathrm{U}_{3} \mathrm{Si}_{2}$ are displayed. The author acknowledges that the peaks shown are very complex, making identification, along with quantitative analysis of secondary U-Si phases, extremely challenging. The characteristic peaks of $\mathrm{UO}_{2}$ are displayed in the graph, as this phase usually occurs upon oxidation of $\mathrm{U}-\mathrm{Si}$ compounds.

Scanning electron microscopy was also performed to further analyze the microstructure of the fabricated samples. A large majority of the sample had a single phase. However, a secondary phase was found by means of electron back scatter detection as displayed in Figure 317. The darker precipitates in the backscatter image, which indicate a higher silicon content, were approximately $5-15 \mu \mathrm{m}$ in length. Energy dispersive spectroscopy proved the existence of a secondary phase composed of $\mathrm{U}$ and $\mathrm{Si}$, which was silicon-rich compared to the surrounding matrix. The phase was assumed to be USi based on accepted phase diagrams [16].

Throughout the U-Si alloys fabrication processes, the element compositions have been designated Si-rich. The reason is to prevent the presence of uranium in solid solution in the alloy and, therefore, in the fuel particles. Such absence will occur if the molten alloy is homogeneous upon solidification and, in the case of alloys, if Si content is from 3.9 to $7.3 \mathrm{wt}$.\%. Since equilibrium, along with a perfect homogeneity, are parameters that can be approached but not accomplished, more than one phase in the fuel alloy should always be expected [1].

\section{Irradiation Performance}

$\mathrm{U}_{3} \mathrm{Si}_{2}$ fuels have demonstrated low levels of swelling occur under irradiation environments, including extremely high burnup. This promising behavior has been attributed to the uniform formation of fission gas bubbles throughout the irradiation process. The fission bubbles tend to remain uniformly distributed along the fuel matrix and do not display signs of coalescence. Additional studies also confirmed that $\mathrm{U}_{3} \mathrm{Si}_{2}$ becomes amorphous during irradiation [24]. The initial rate of swelling in $\mathrm{U}_{3} \mathrm{Si}_{2}$ is relatively low, and then it drastically increases. Such 
transition, identified as "knee point," shows the fission density in which fission gas bubbles reach a size large enough to influence the swelling behavior. Prior to the knee point, a relatively small fraction of fission gas is retained in solution and the rest in nanoscale-sized bubbles that SEM cannot identify [24].

In addition, computer simulations employing cutting-edge models for fission gas behavior have proven that bubble-size distribution is bimodal. Besides the larger and noticeable bubbles formed on dislocations, there exists a population of much smaller bubbles in the matrix [25]. Figure 3-18 shows the swelling behavior as a function of fission density for different fuels. The high-density fuel candidates displayed unacceptable swelling rates at low-and mediumfission densities. The silicide compounds, $\mathrm{U}_{3} \mathrm{Si}_{2}$ and $\mathrm{U}_{3} \mathrm{Si}$, showed swelling rates that were fairly low in relation to the rest of the examined fuels. Based on their swelling behavior, these two candidates were categorized as stable and acceptable accident-tolerant fuels. The main differences among $\mathrm{U}_{3} \mathrm{Si}_{2}$ and $\mathrm{U}_{3} \mathrm{Si}$ are the swelling behavior and the fission gas bubble morphology.

$\mathrm{U}_{3} \mathrm{Si}_{2}$ is a stable compound in which fission gas bubble morphology is uniformly displayed with no evidence of bubble coalescence. The small bubbles formed remained stable to high burnups. In the study, the low-level swelling in $\mathrm{U}_{3} \mathrm{Si}_{2}$ was attributed to a uniform bimodal distribution of fission gas bubbles that did not display any interlinkage [26]. Fission gas bubbles in the visible range form after fission densities of approximately $3.5 \times 10^{17}$ fissions $/ \mathrm{cm}^{3}$ increase the swelling rate. Due to the small bubble formation, the swelling is clearly less pronounced in $\mathrm{U}_{3} \mathrm{Si}_{2}$ than in $\mathrm{U}_{3} \mathrm{Si}$. The end result is the absence of pillowing and break-away swelling, even at full burnup scenarios [25]. Such behavior suggested that an underlying microstructure was responsible for the observed behavior. Figure 3-19 displays the microstructure of an high 
enriched uranium (HEU) sample (93\% $\mathrm{U}^{235}$ enrichment) at a burnup of 63\%, in which the fission gas bubbles conserve a uniform morphology [24].

The swelling behavior in $\mathrm{U}_{3} \mathrm{Si}$ drastically differs from that in $\mathrm{U}_{3} \mathrm{Si}_{2}$. Figure 3-20 displays the fission gas bubble formation for both $\mathrm{U}_{3} \mathrm{Si}$ and $\mathrm{U}_{3} \mathrm{Si}_{2}$. In $\mathrm{U}_{3} \mathrm{Si}_{2}$, the swelling rate is not only higher at early irradiation stages, but it intensifies during the middle stages, resulting in fuel plate pillowing. Such swelling is attributed to the evolution of bubble morphology. Bubbles in $\mathrm{U}_{3} \mathrm{Si}$ do not conserve a uniform formation pattern. Instead, they have a wide variety of shapes and sizes. They also interlink to form larger cavities [26]. It has been proposed that the high bubble growth rate occurs due to the amorphous transformation of $\mathrm{U}_{3} \mathrm{Si}$ during irradiation. Fission in the amorphous compound vastly increases diffusion and reduces the plastic flow strength [26].

Additional studies indicated that if fission gas bubbles in $\mathrm{U}_{3} \mathrm{Si}_{2}$ fuel had grown at a constant rate, the distribution would have remained uniform over all fission densities; however, the distribution was developed in a non-uniform manner, as displayed in Figure 3-21. The total number of bubbles decreased as a function of fission density. Bubbles seemed to form at different rates, suggesting that bubble interlinking had occurred during the process [24]. Fission gas bubble density and distribution are highly influenced by the fission rate and coalescence. The fission rate is a main factor affecting swelling behavior. Nonetheless, the varying chemistry of the fuel is assumed to exert a strong influence on the behavior. As a result, the swelling beyond the knee point is understood to be non-linear [24]. 
Table 3-1. Plasma formation gains and losses [11].

\begin{tabular}{ll}
\hline Energy Gain & Energy Loss \\
\hline Inter-band absorption & Carrier Diffusion \\
Free carrier absorption & Phonon emission \\
& Direct Recombination by photon \\
& emission \\
\hline
\end{tabular}

Table 3-2. Density of different accident-tolerant fuel candidates [17].

\begin{tabular}{lccccccc}
\hline Uranium Compound & $\mathrm{UAI}_{4}$ & $\mathrm{UAI}_{3}$ & $\mathrm{UAI}_{2}$ & $\mathrm{U}_{3} \mathrm{O}_{8}$ & $\mathrm{USi}$ & $\mathrm{U}_{3} \mathrm{Si}_{2}$ & $\mathrm{U}_{3} \mathrm{Si}$ \\
\hline Theoretical Density $\left(\mathrm{g} / \mathrm{cm}^{3}\right)$ & 6.1 & 6.8 & 8.1 & 8.3 & 11.0 & 12.2 & 15.4
\end{tabular}

Table 3-3. Key properties of $\mathrm{UO}_{2}$ and $\mathrm{U}_{3} \mathrm{Si}_{2}$ [14].

\begin{tabular}{lcc}
\hline \multicolumn{1}{c}{ Property } & $\mathrm{UO}_{2}$ & $\mathrm{U}_{3} \mathrm{Si}_{2}$ \\
\hline Theoretical Density $\left(\mathrm{g} / \mathrm{cm}^{3}\right)$ & 10.96 & 12.2 \\
Theoretical Uranium number density & $2.44 \times 10^{22}$ & $2.86 \times 10^{22}$ \\
$\left(\right.$ atom $\left./ \mathrm{cm}^{3}\right)$ & & \\
Thermal Conductivity $\left(\mathrm{W} / \mathrm{m} \mathrm{K} 400-1200^{\circ} \mathrm{C}\right)$ & $6-2.5$ & $13-22.3$ \\
Melting Point $\left({ }^{\circ} \mathrm{C}\right)$ & 2,847 & 1,665 \\
\hline
\end{tabular}


Table 3-4. Crystallographic and thermodynamic data for the U-Si system [18].

\begin{tabular}{|c|c|c|c|c|c|c|c|}
\hline Milling Time & $0 \mathrm{~h}$ & $5 \min$ & $1 \mathrm{~h}$ & $3.5 \mathrm{~h}$ & $8 \mathrm{~h}$ & $12 \mathrm{~h}$ & $24 \mathrm{~h}$ \\
\hline Phase Content & $\mathrm{U}+\mathrm{Si}$ & $\begin{array}{c}\mathrm{U}+\mathrm{Si}+\mathrm{U} \\
\mathrm{Si}_{3}\end{array}$ & $\mathrm{U}+\mathrm{USi}_{3}$ & $\begin{array}{l}\text { U+USi3 } \\
+ \text { Minor }\end{array}$ & $\mathrm{USi}_{3}$ & $\mathrm{U}_{3} \mathrm{Si}_{2}$ & $\mathrm{U}_{3} \mathrm{Si}_{2}$ \\
\hline Free Powder (g) & 5 & 5 & 5 & 4.99 & 3.93 & 3.89 & 3.76 \\
\hline Free Powder (\%) & 100 & 100 & 100 & 98.3 & 70.2 & - & 64.4 \\
\hline Particle Size $(\mu \mathrm{m})$ & 100 & 100 & $50-200$ & 50-200 & $10-300$ & $10-50$ & $10-50$ \\
\hline $\begin{array}{l}\text { Particle } \\
\text { Morphology }\end{array}$ & Spherical & Spherical & Laminate & Platelet & Irregular & Equiaxed & Equiaxed \\
\hline
\end{tabular}

Table 3-5. Chemical analysis conducted on comminuted $\mathrm{U}_{3} \mathrm{Si}_{2}$ buttons. Elements present in the analysis but below the detection limit include: $\mathrm{Al}, \mathrm{Sb}, \mathrm{Ba}, \mathrm{Be}, \mathrm{Cd}, \mathrm{Ca}, \mathrm{Cu}, \mathrm{Pb}, \mathrm{Li}, \mathrm{Mg}$, Mn, Mo, Ni, P, K, Se, Na, Sr, Sn, Ti, Zn, Zr, B, Gd, Nb, Ta, and W [16].

\begin{tabular}{lccccccc}
\hline Elements & $\mathrm{Si}$ & $\mathrm{C}$ & $\mathrm{H}$ & $\mathrm{N}$ & $\mathrm{Fe}$ & $\mathrm{As}$ & $\mathrm{Cr}$ \\
\hline $\begin{array}{l}\text { Contents } \\
\text { (w/o) }\end{array}$ & 7.28 & 0.08 & 0.27 & 0.12 & 0.04 & 0.03 & 0.006 \\
\hline
\end{tabular}


A

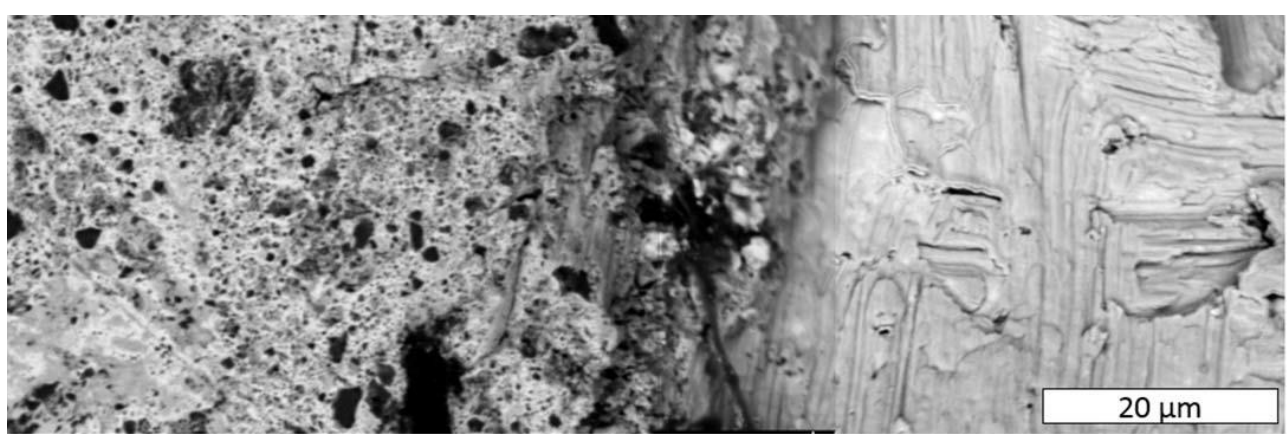

$\mathrm{B}$
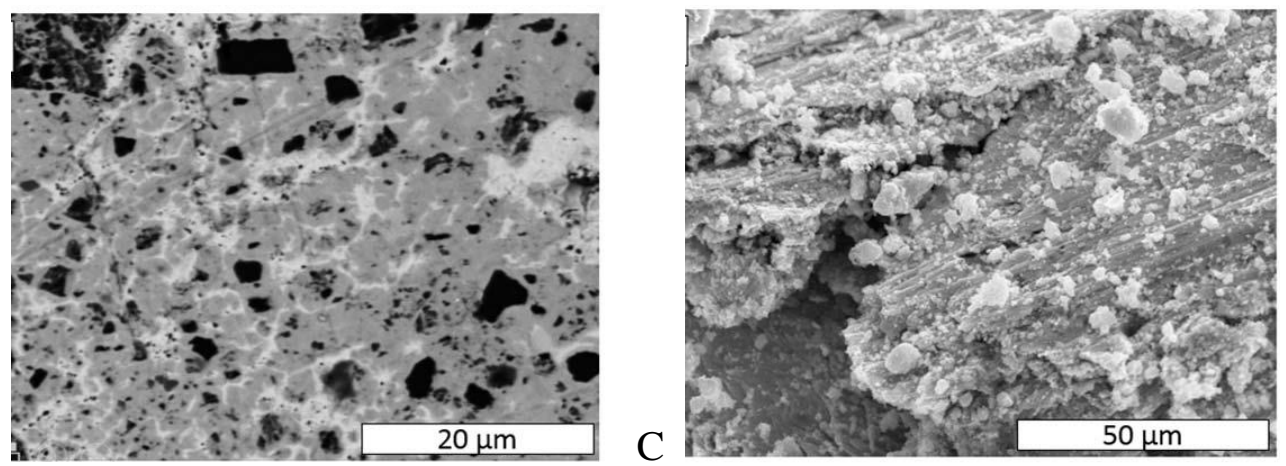

Figure 3-1. Microstructural SEM images from $\mathrm{Ce}_{3} \mathrm{Si}_{2}$ synthesis. A) Sectioned and polished fragment recovered after MSR. On the right side, Ce exterior displays melting during reaction; shifting toward the left, the inhomogeneous interior is displayed. B) Ce, $\mathrm{Ce}_{3} \mathrm{Si}_{2}$, and silicon on a three-phase region. C) SEM image prior to MSR displaying Si particles layered with Ce sheets [6].

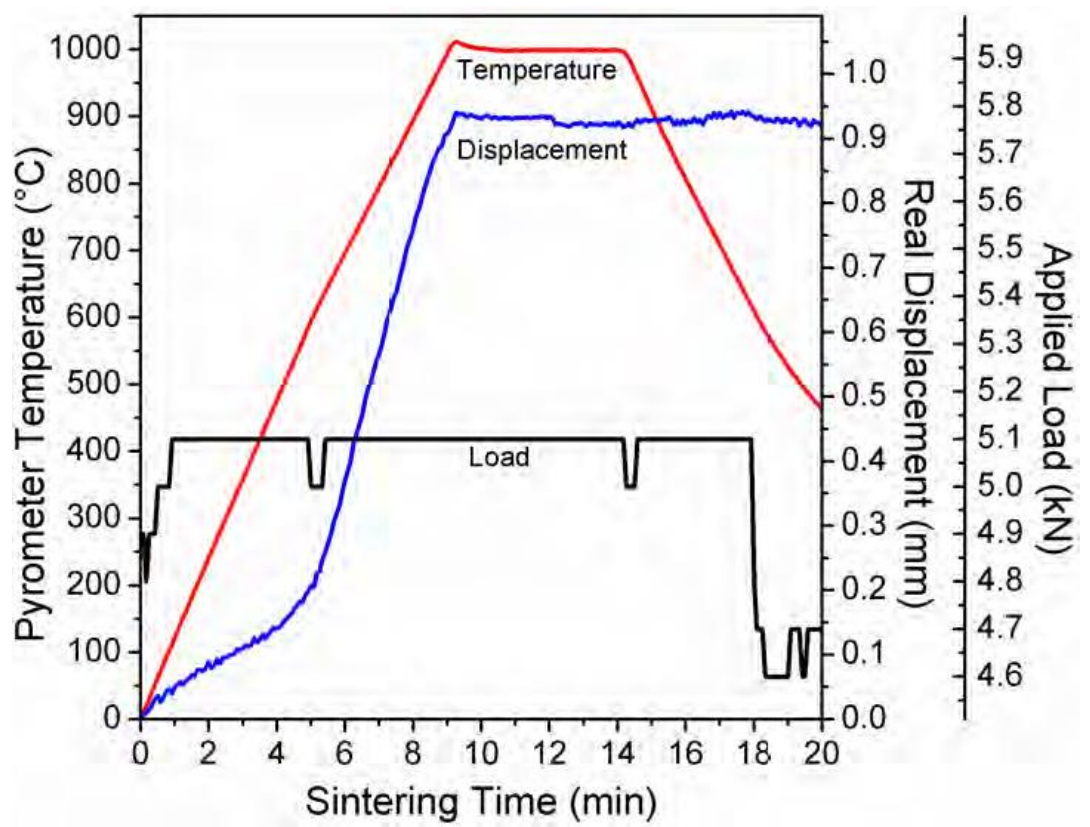

Figure 3-2. Typical spark plasma sintering experiment conducted with as-milled $\mathrm{Ce}_{3} \mathrm{Si}_{2}$ powder [6]. 


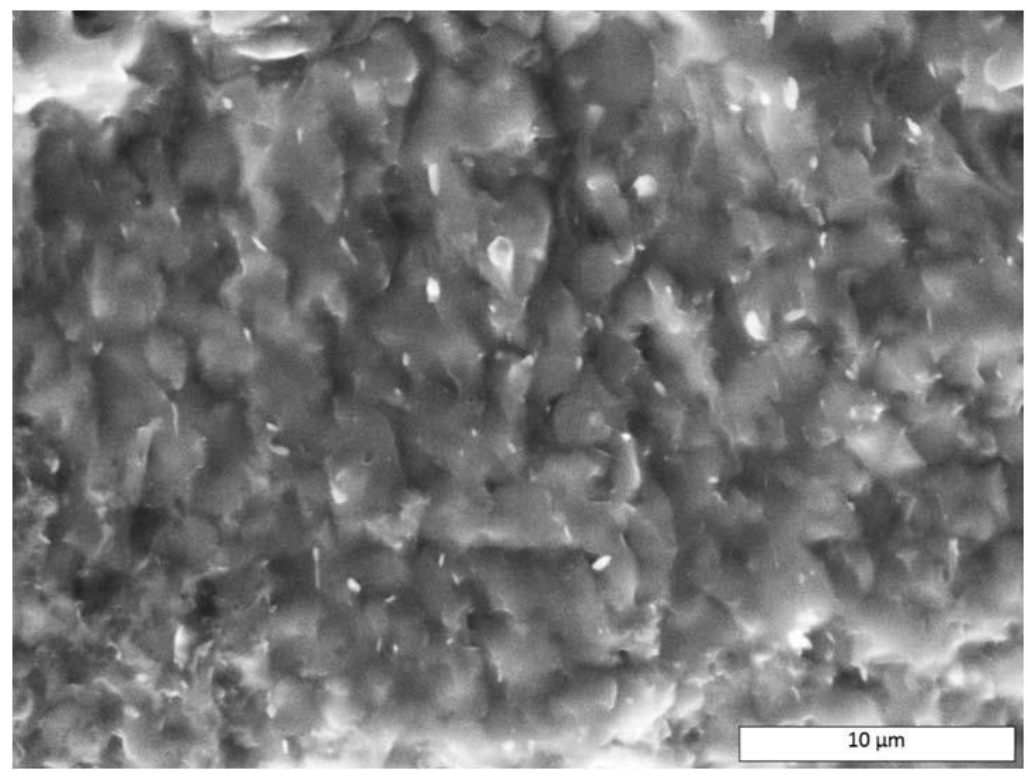

Figure 3-3. SPS sintered $\mathrm{Ce}_{3} \mathrm{Si}_{2}$ displaying a few small, rounded pores at grain boundaries [6].

A
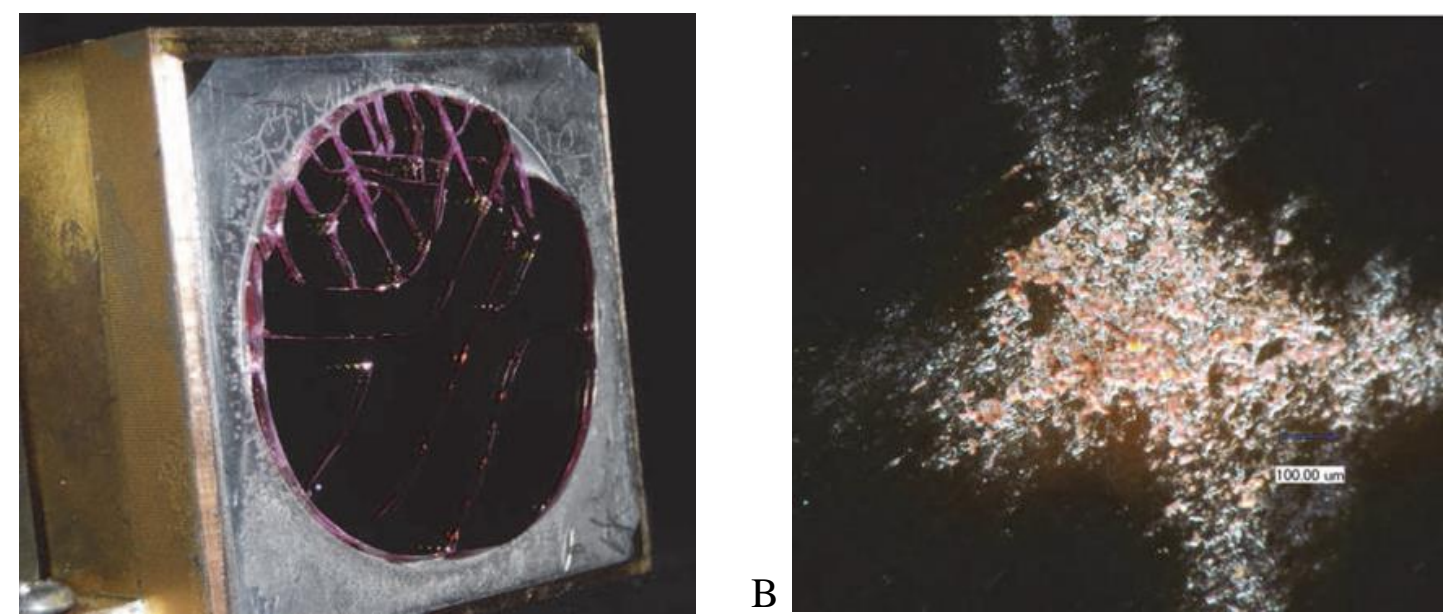

Figure 3-4. Laser material interaction effects. A) Thermal fractures of Nd:YAG (neodymiumdoped yttrium aluminium garnet) and $\mathrm{B}$ ) melting of $\mathrm{SiO}_{2}$ thin film coated on $\mathrm{Nd}$ :YAG in a high-energy laser [10]. 


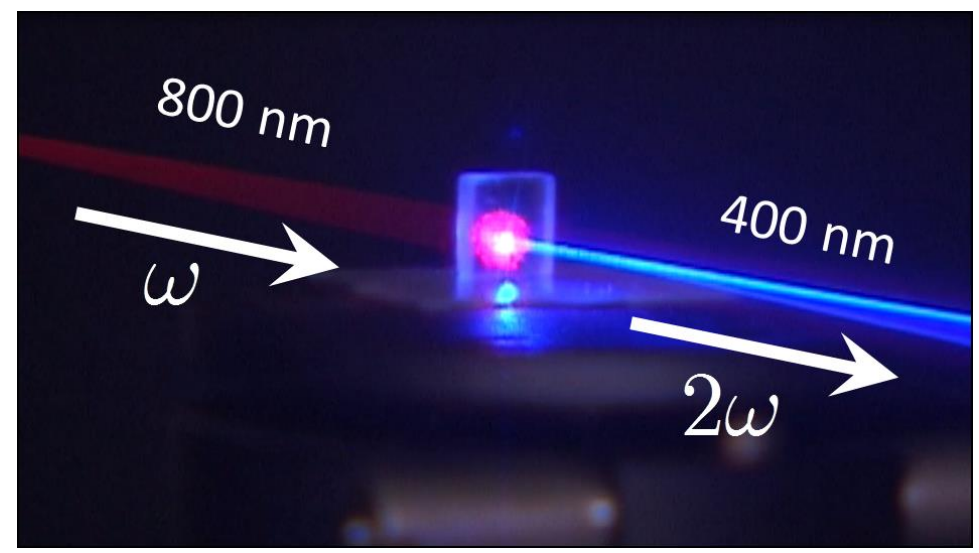

Figure 3-5. Second harmonic generation process of incident beam of frequency $\omega$ [12].
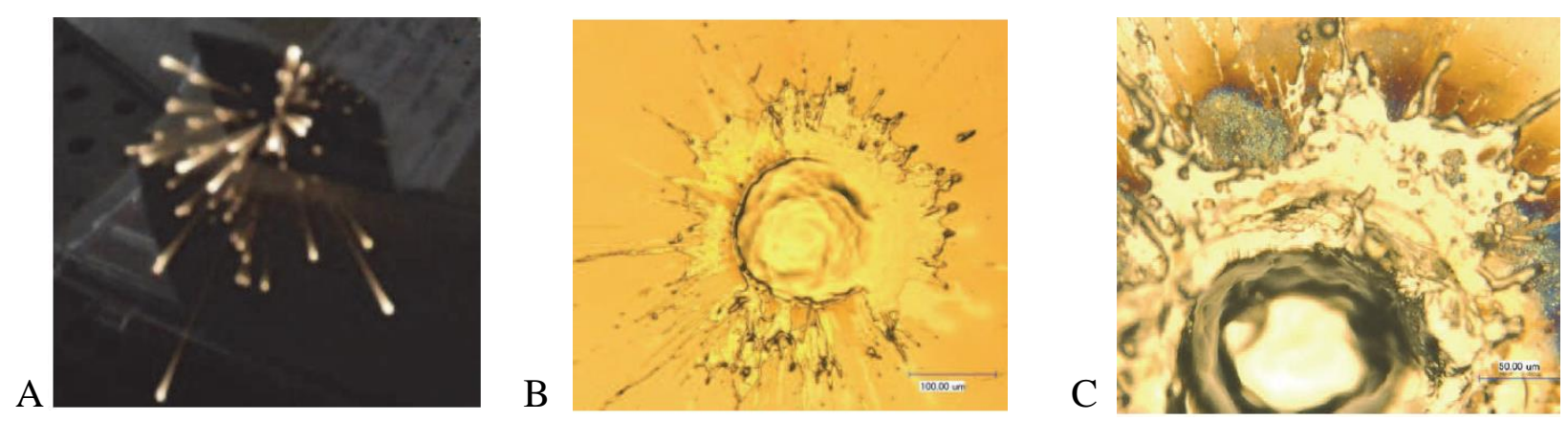

Figure 3-6. The laser energy damage generated in a polycrystalline silicon target. A) Sputtering of laser-induced plasma. B) Damaged area displaying a crater and sputtering in the surroundings. C) Damage morphology generated with a $7 \mathrm{~Hz}$ repetition rate laser energy beam (1 second exposure) [10]. 


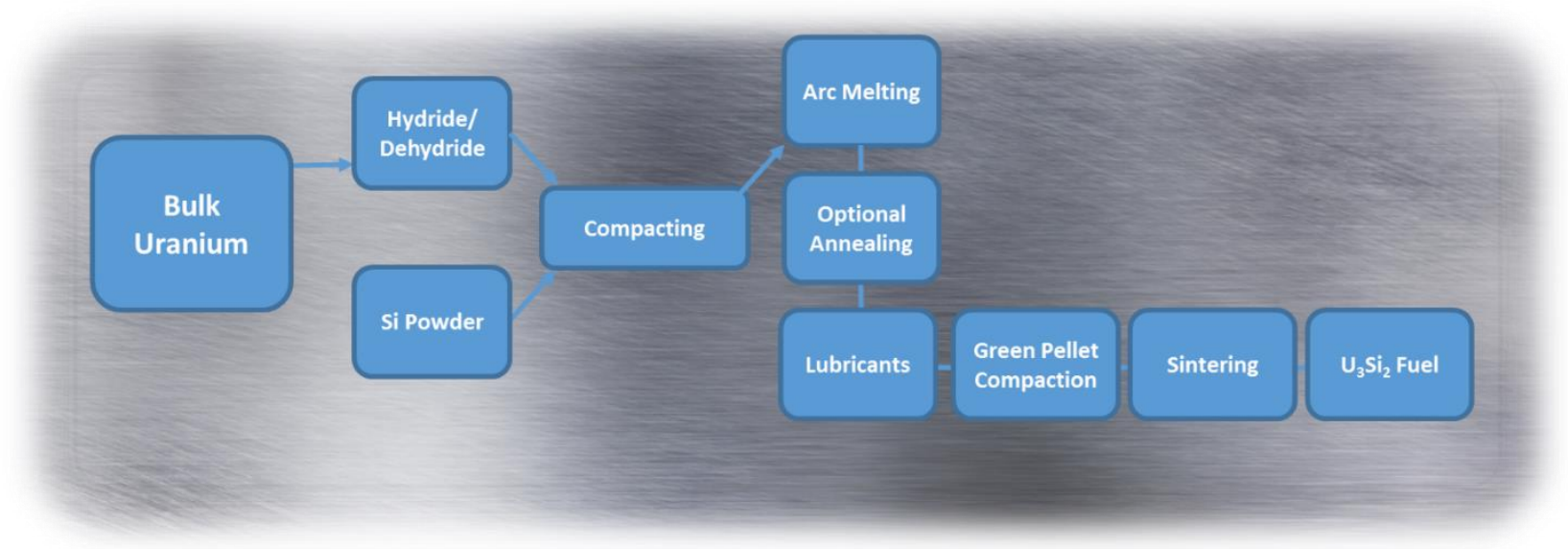

Figure 3-7. $\mathrm{U}_{3} \mathrm{Si}_{2}$ fuel fabrication process on a laboratory scale.

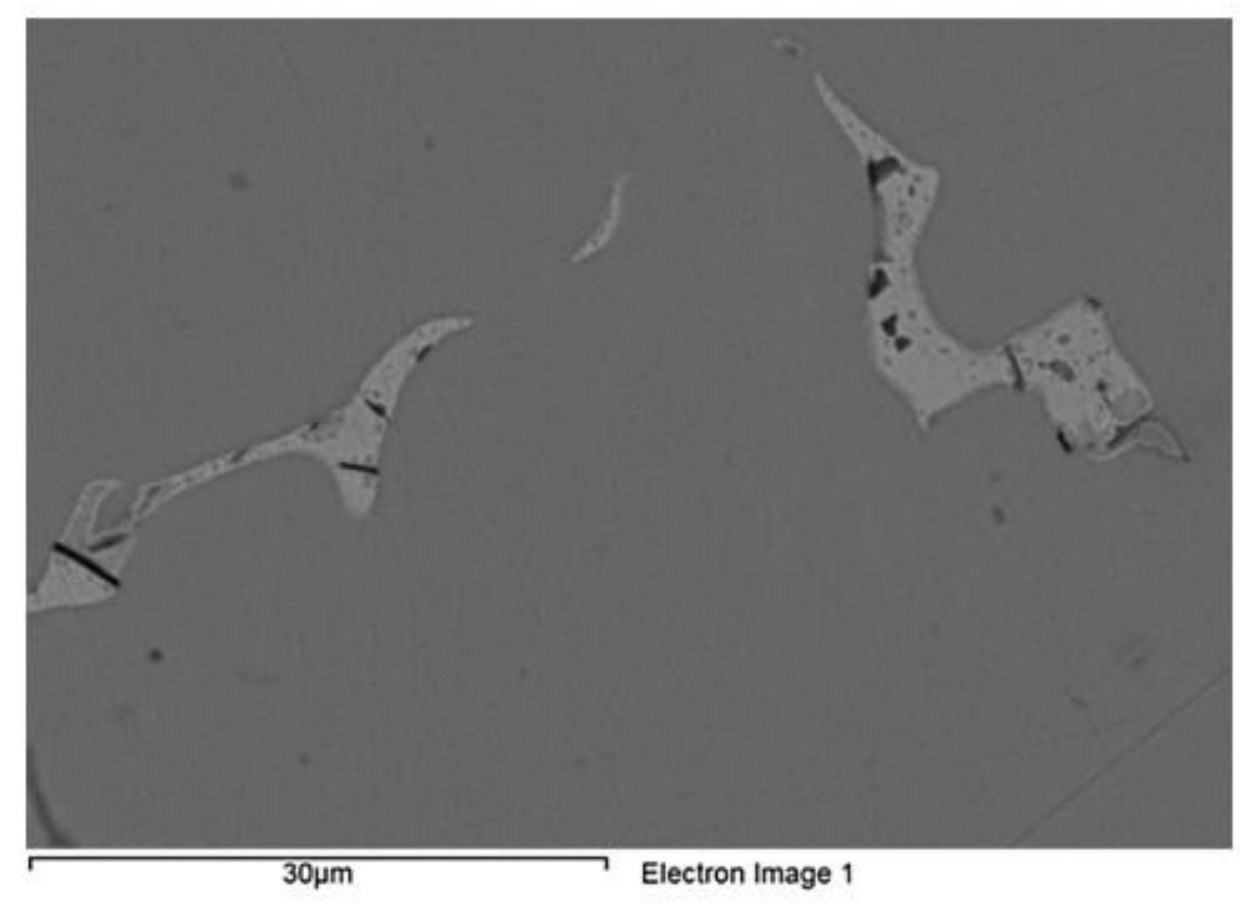

Figure 3-8. Electron backscatter image of $\mathrm{U}_{3} \mathrm{Si}_{2}$ inclusions in arc melted uranium silicide [14]. 


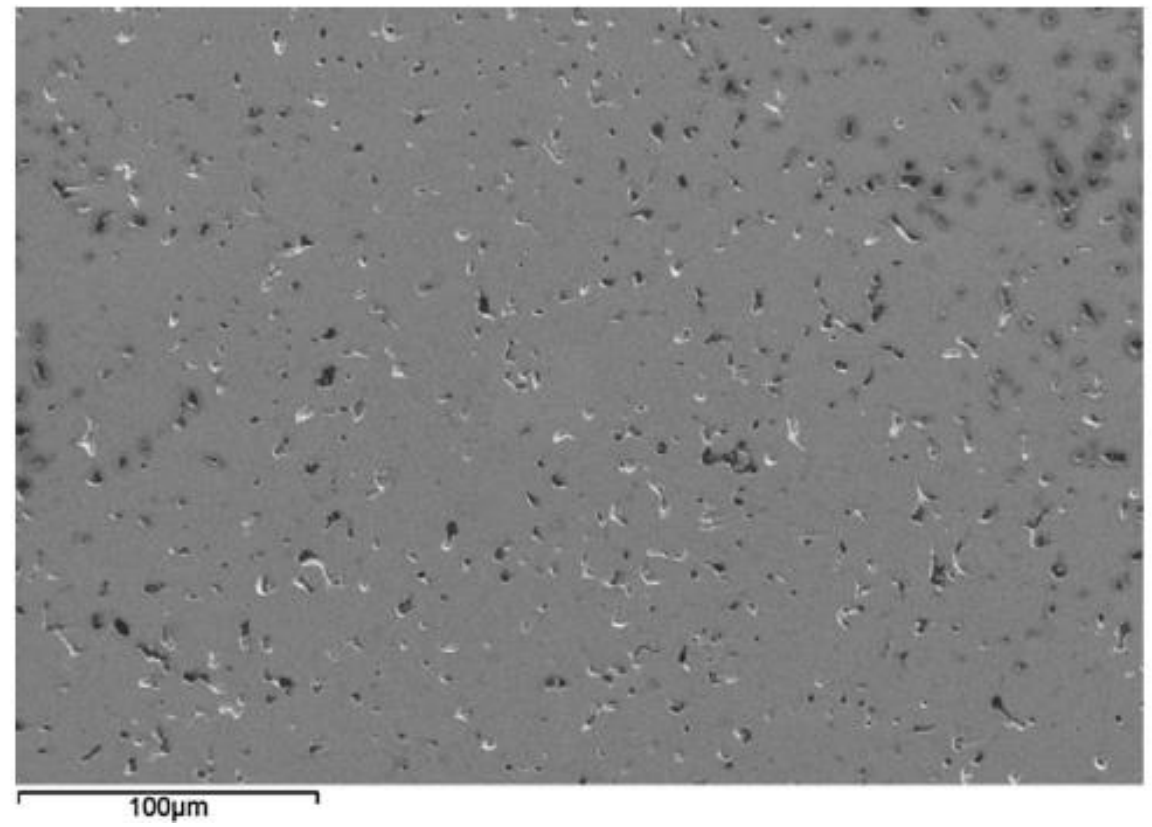

Figure 3-9. SEM image displaying the sintered microstructure of $\mathrm{U}_{3} \mathrm{Si}_{2}$ [14].

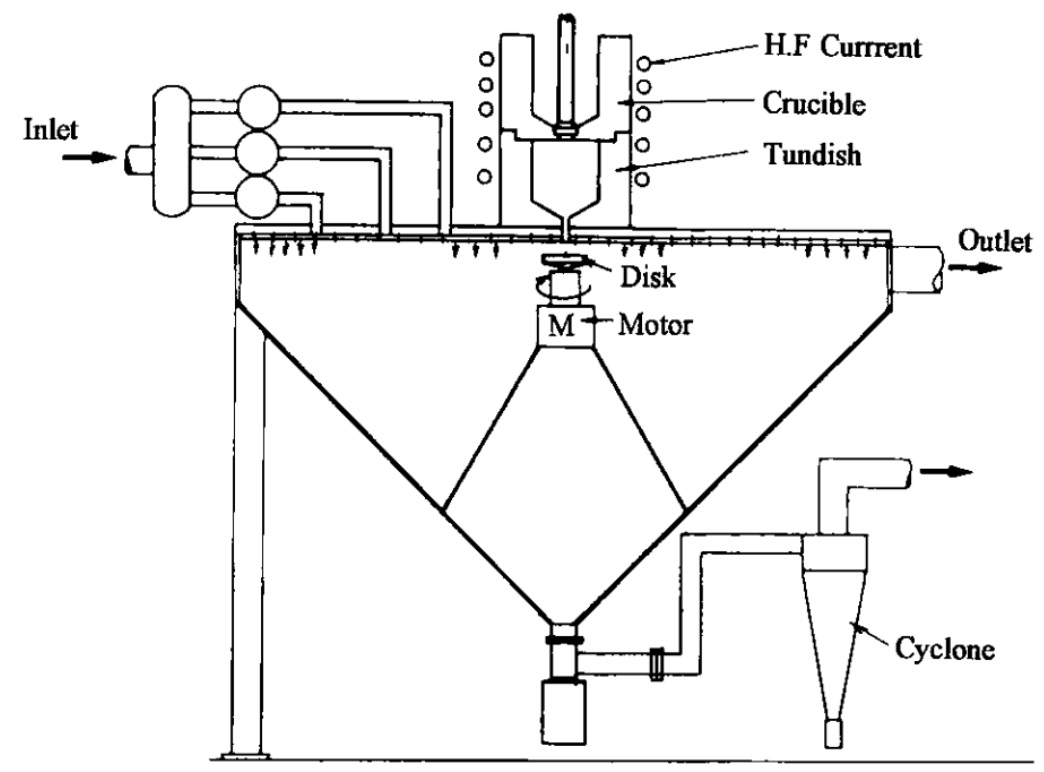

Figure 3-10. A schematic view of the centrifugal atomizer apparatus [19]. 


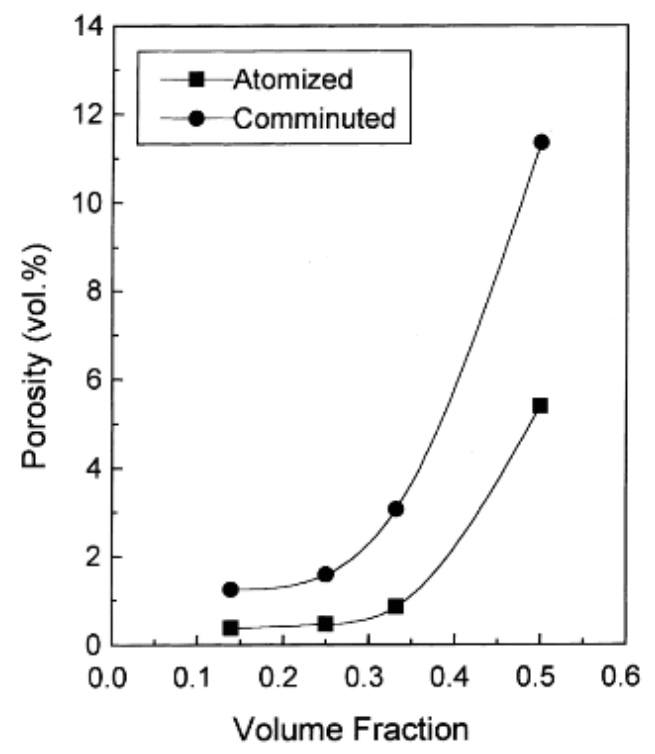

Figure 3-11. Void content of extruded fuel cores with comminuted and atomized $\mathrm{U}_{3} \mathrm{Si}_{2}$ particles [18].
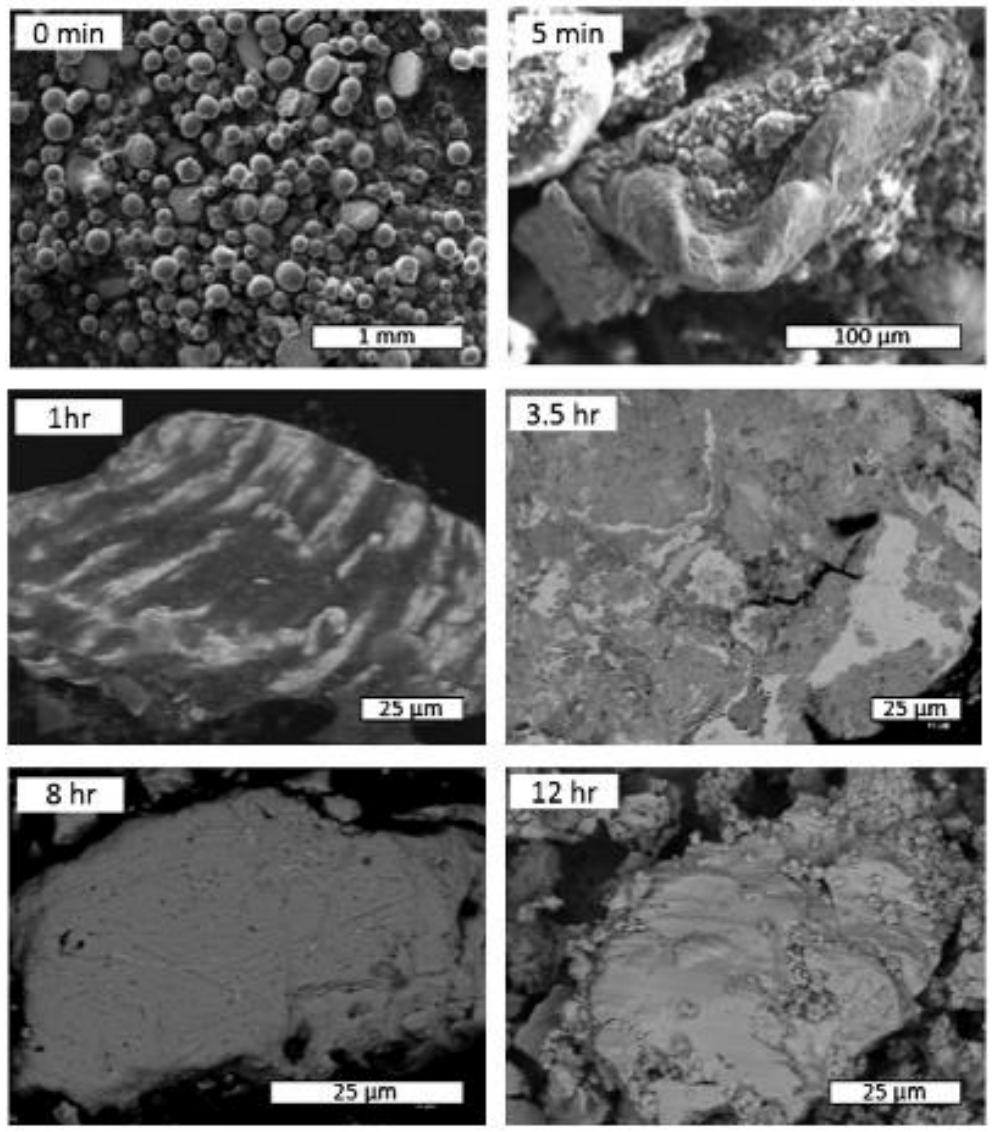

Figure 3-12. Representative micrographs from the specified milling times show microstructure development with increasing milling time. 0 and 5 min images are SEM micrographs, while $1-12 \mathrm{~h}$ images show phase contrast with BSE micrographs [18]. 


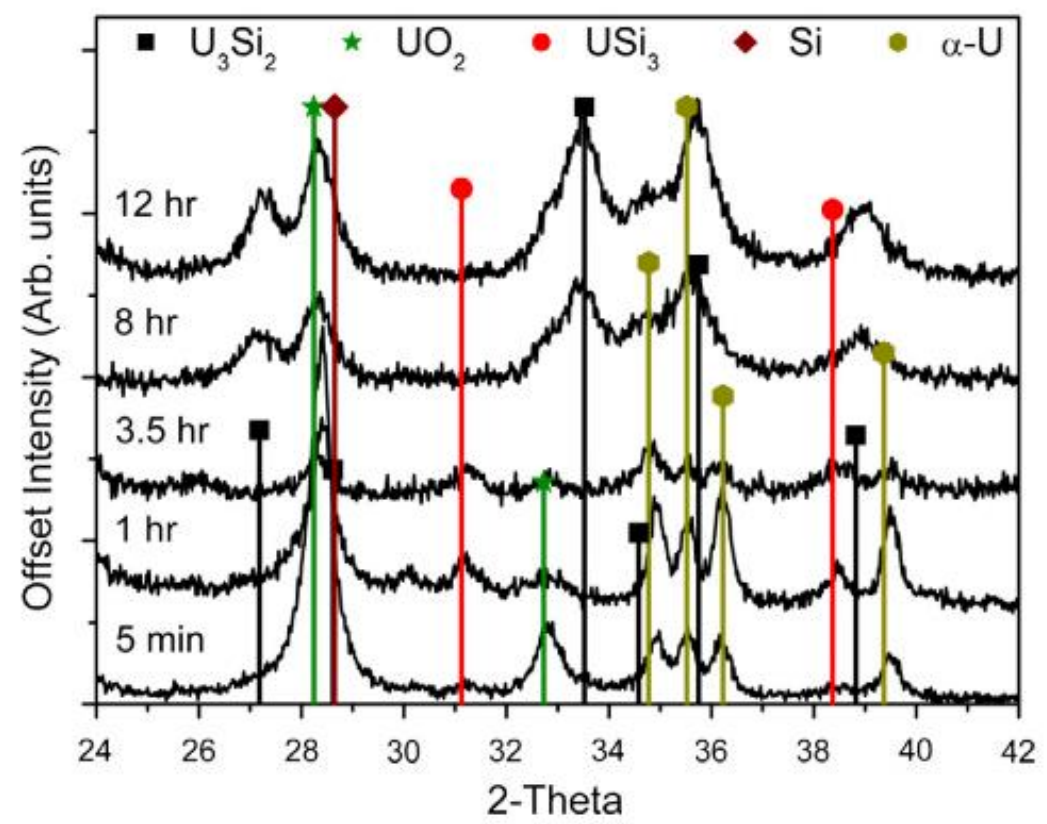

Figure 3-13. Representative XRD $2 \theta$ scans for $\mathrm{U}_{3} \mathrm{Si}_{2}$ milled in clean vessels with CSZ media for times from 5 min to 12 hours [18].

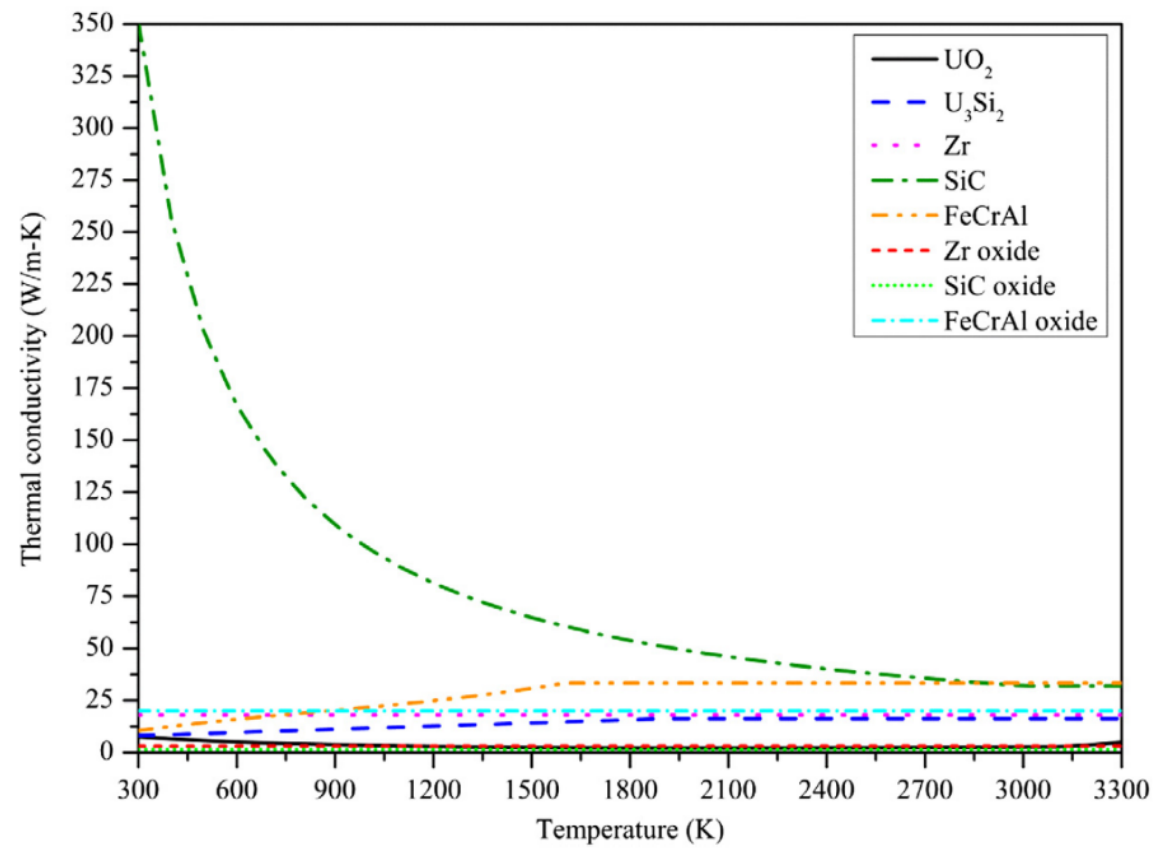

Figure 3-14. Thermal conductivity of fuel and cladding materials [22]. 


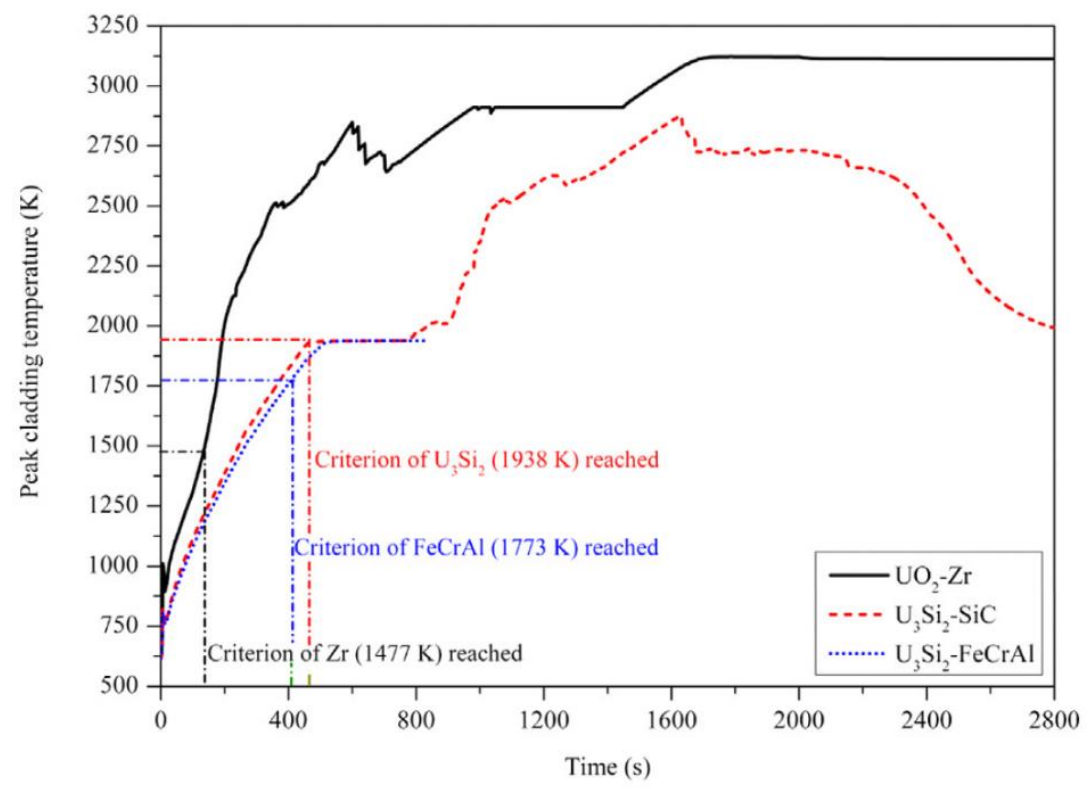

Figure 3-15. Peak cladding temperature for LBLOCA [22].

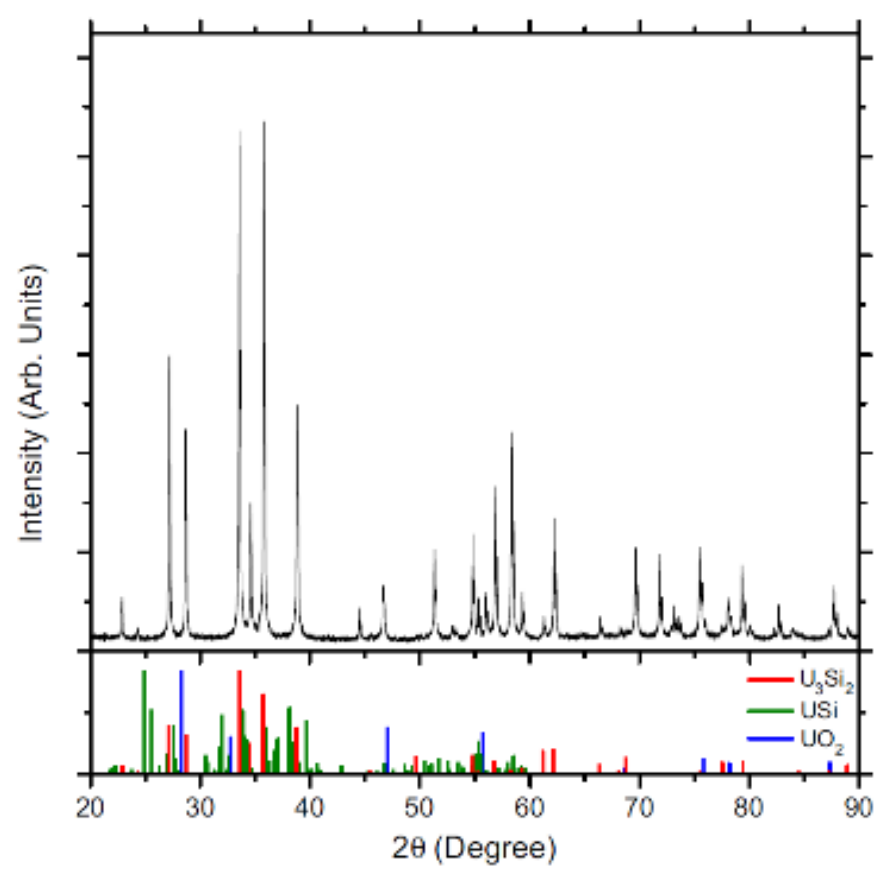

Figure 3-16. X-Ray diffraction pattern of a $\mathrm{U}_{3} \mathrm{Si}_{2}$ sample [16]. 

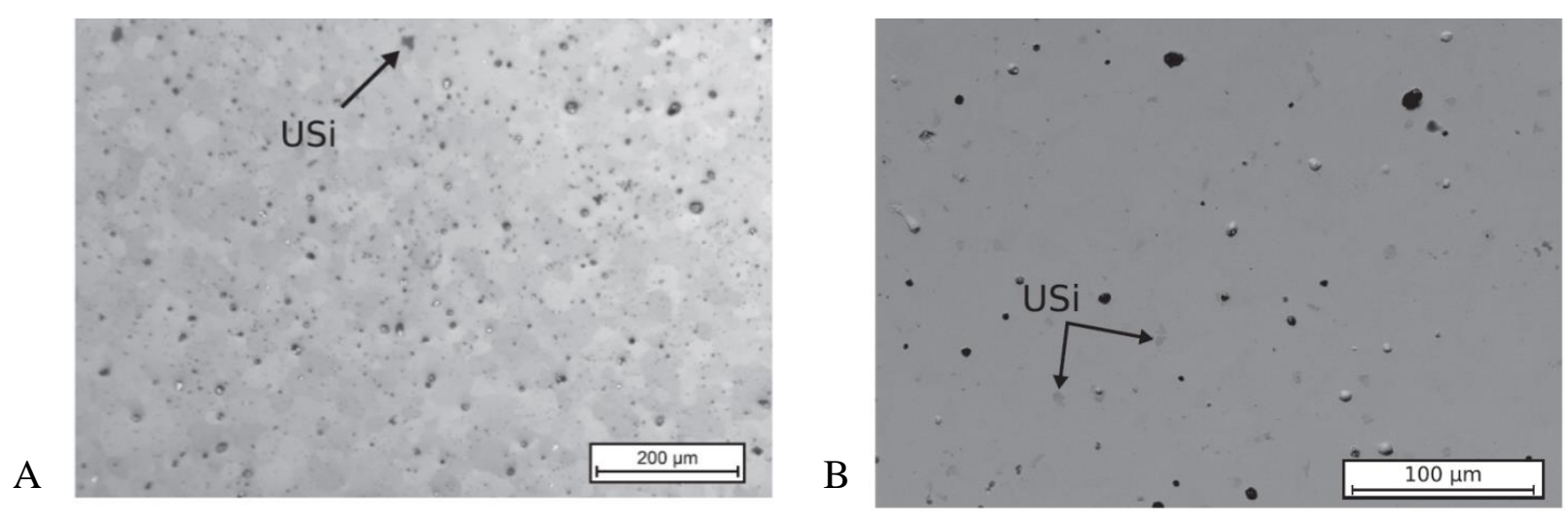

Figure 3-17. Microstructure of a $\mathrm{U}_{3} \mathrm{Si}_{2}$ sample. A) Displays the matrix composed of a $\mathrm{U}_{3} \mathrm{Si}_{2}$ phase along with a secondary phase present (USi). B) The morphology of a $\mathrm{U}_{3} \mathrm{Si}_{2}$ sample was displayed employing backscatter electron imaging [16].

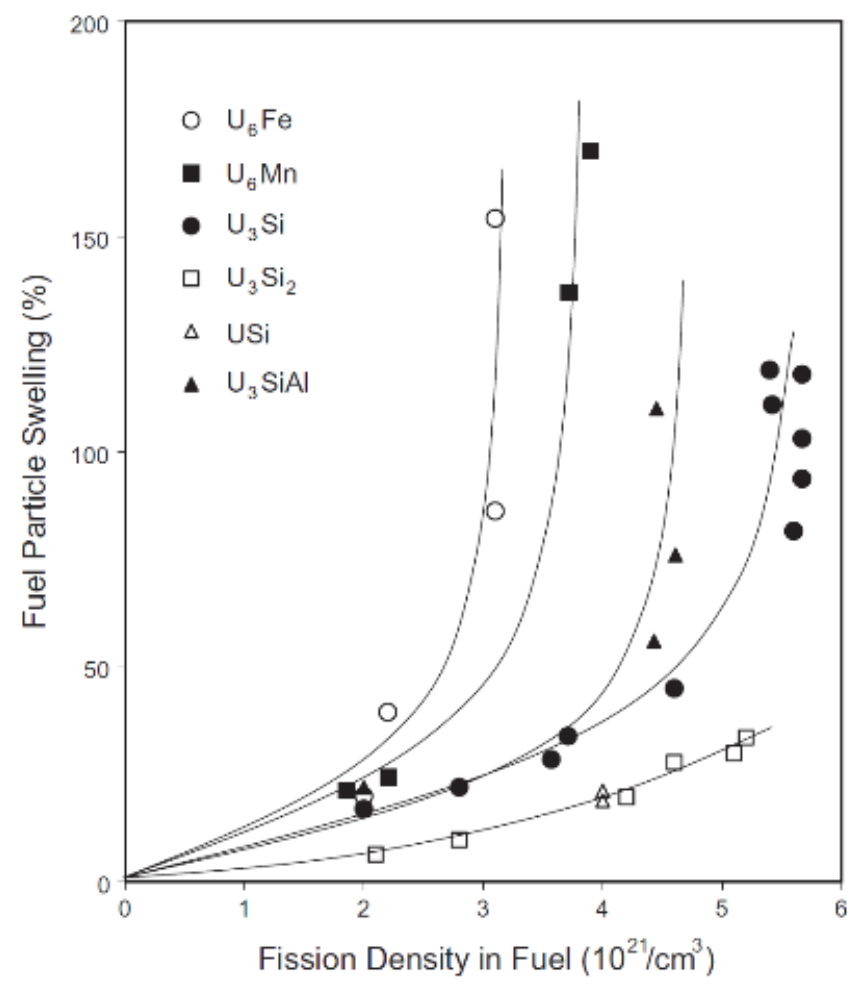

Figure 3-18. Fuel particle swelling as a function of fission density [24]. 


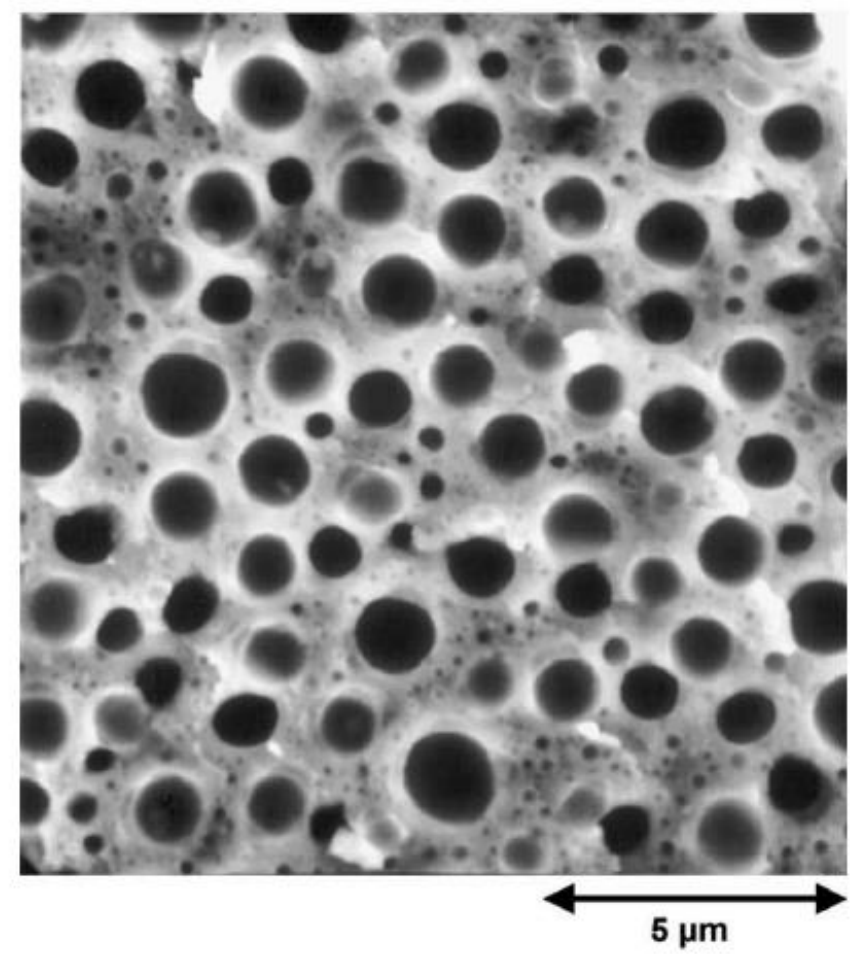

Figure 3-19. SEM micrograph of a $\mathrm{U}_{3} \mathrm{Si}_{2} \mathrm{HEU}$ fuel particle at a fission density of $14.3 \times 10^{21}$ $\mathrm{f} / \mathrm{cm}^{3}[24]$.

A

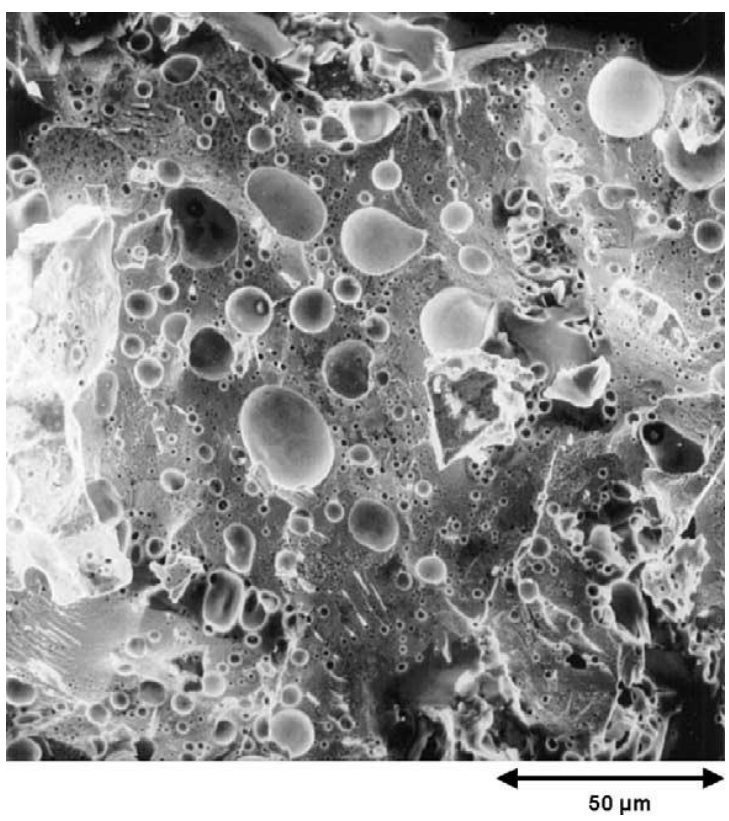

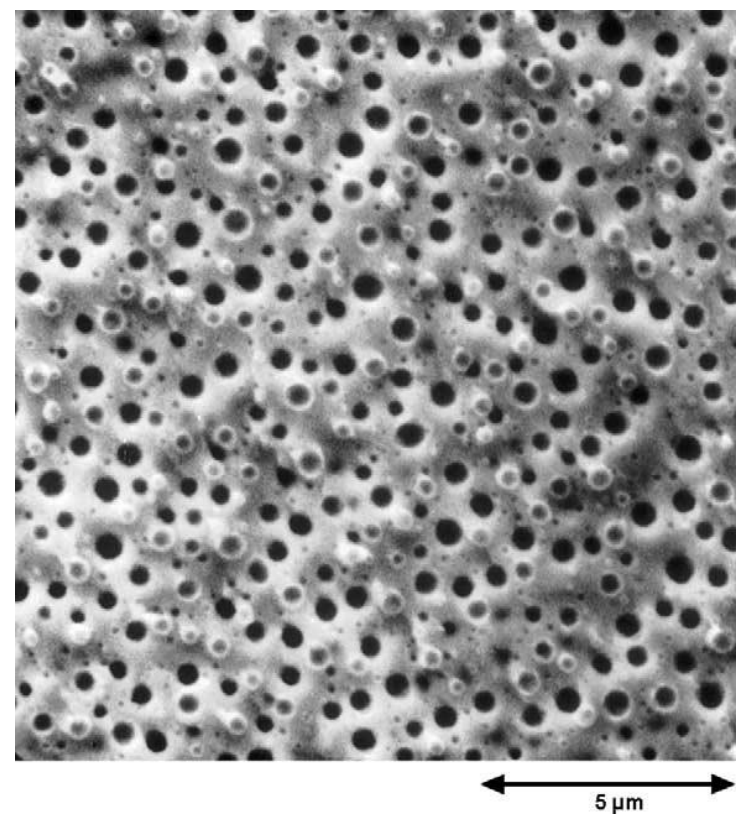

s. A) Fission gas bubble morphology in . B) $\mathrm{U}_{3} \mathrm{Si}_{2}$ sample at $96 \%$ burnup, $5.2 \times$ $\mathrm{U}_{3} \mathrm{Si}$ at $73 \%$ burnup, $4.3 \times 1021$ fissions $/ \mathrm{cm}^{3}$. $10^{21}$ fissions $/ \mathrm{cm}^{3}[24]$. 

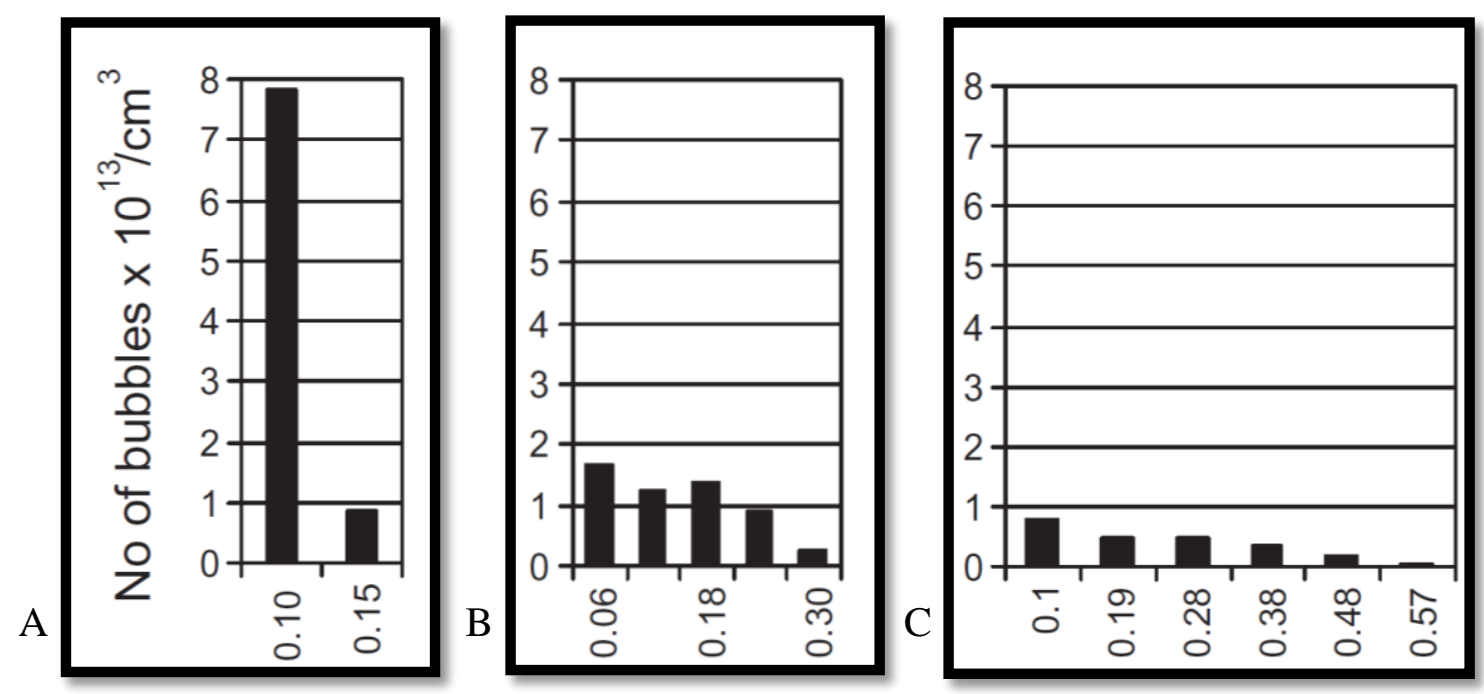

Figure 3-21. Distribution and number of fission gas bubbles in $\mathrm{U}_{3} \mathrm{Si}_{2}$ fuel at fission densities of A) $2.8 \times 10^{21} \mathrm{f} / \mathrm{cm}^{3}$, B) $4.2 \times 10^{21} \mathrm{f} / \mathrm{cm}^{3}$, and C) $4.6 \times 10^{21} \mathrm{f} / \mathrm{cm}^{3}[24]$. 


\section{CHAPTER 4 \\ A THERMODYNAMIC ANALYSIS - UNDERSTANDING SURROGATE FUEL FORMATION}

\section{Background}

A thermodynamic study assisted to understand the kinetics of formation of $\mathrm{U}_{3} \mathrm{Si}_{2}$ and other surrogate silicide fuels. Thermodynamic elemental principles were included in the analysis as they strictly regulate heat transformations in materials. Additionally, a survey of thermodynamic and mechanical properties of potential compounds was conducted to examine the suitability of the materials to undergo a laser synthesis. Some of the relevant properties evaluated were the crystallographic structure to ensure similar behavior of the surrogate material to that of $\mathrm{U}_{3} \mathrm{Si}_{2}$. The enthalpy along with the Gibbs free energy of formation assisted to determine the spontaneity of the reactions to form the desired compounds $\left(\mathrm{Ce}_{3} \mathrm{Si}_{2}, \mathrm{Zr}_{3} \mathrm{Si}_{2}\right.$, and $\mathrm{Hf}_{3} \mathrm{Si}_{2}$ ). In addition, the melting points of the compounds were evaluated to adjust the laser parameters for optimum synthesis. The properties evaluated are displayed on Table 4-1.

The phase diagrams of the different surrogate materials were analyzed to have a thorough understanding of the specific stoichiometry and temperatures required to form the desired compounds $\left(\mathrm{Ce}_{3} \mathrm{Si}_{2}, \mathrm{Zr}_{3} \mathrm{Si}_{2}\right.$, and $\left.\mathrm{Hf}_{3} \mathrm{Si}_{2}\right)$. The aforementioned thermodynamic studies were performed in order to comply with Objective 1 (See Chapter 1, pg. 21).

\section{Thermodynamics}

\section{First Law of Thermodynamics}

The first law of thermodynamics is the principle of the conservation of energy involving the production or absorption of heat [27]. The first law states that energy cannot be created nor destroyed, energy can only be transferred or changed from one form to another. For any process, there is a function of state, called the internal energy, $U$, which depends solely on the initial and final thermodynamic coordinates of the system. Differences in this quantity may be determined 
by calculating or measuring the work performed adiabatically on the system for the process under study [28]. The first law is mathematically expressed in Equation 4-1 where $\Delta U$ is the change in energy corresponding to a given transformation in the system, $Q$ is the energy added to the system in the form of heat (heat transfer), and $W$ is the work performed by the system on its surroundings.

$$
\Delta \mathrm{U}=Q+W
$$

The value of $\Delta \mathrm{U}$ is the change in energy accompanying a transition from state 1 into state 2 and can be expressed as $U_{2}-U_{1}$. The change in state is independent of the manner of the transition from state 1 to state 2 . The decrease of energy in a system subjected to any change is the mechanical equivalent of the external effects resulting from such change [28].

\section{Second Law of Thermodynamics}

The second law of thermodynamics places a constraint on the direction of heat transfer.

The law states the existence of the state variable entropy, $S$. The change in entropy $\Delta S$ is equal to the heat transfer $Q$ divided by the temperature $T$ as displayed in equation 4-2 [29].

$$
\Delta \mathrm{S}=\frac{\Delta \mathrm{Q}}{T}
$$

In a physical process, both the entropy of the system and of the environment remain constant if the process can be reversed. Denoting the initial and final states with "i" and "f", respectively. In the case that the physical process is irreversible, the combined entropy of the system and environment shall increase. Hence, the final entropy will be greater than the initial entropy during an irreversible process [29].

$$
\begin{array}{ll}
\mathrm{S}_{\mathrm{f}}=\mathrm{S}_{\mathrm{i}} & \text { Reversible process } \\
\mathrm{S}_{\mathrm{f}}>\mathrm{S}_{\mathrm{i}} & \text { Irreversible process }
\end{array}
$$


The postulate of Clausius for the second law states that "A transformation whose only final result is to transfer heat from a body at a given temperature to a body at a higher temperature is impossible" [30].

\section{Third Law of Thermodynamics}

The third law is a postulate that was advanced by Walter Nernst, Max Planck, and others. The postulate states that the entropy of all homogeneous materials can be taken as zero at the absolute zero temperature (0 Kelvin). At this temperature the calculation of the absolute values of entropy of pure substances can be performed solely from their heat capacity [30].

\section{Gibbs Free Energy}

The Gibbs free energy is generally denoted by $G$ and combines enthalpy and entropy into a single value. The Gibbs free energy $G$ is equal to the enthalpy of the system $H$, minus the product of the temperature T, and the entropy $S$ of the system, as displayed by Equation 4-3 [31].

$$
\mathrm{G}=\mathrm{H}-\mathrm{TS}
$$

Then taking the differential one arrives at Equation 4-4.

$$
d \mathrm{G}=d \mathrm{H}-\mathrm{S} d \mathrm{~T}-\mathrm{T} d \mathrm{~S}
$$

At a constant temperature the later expression reduces to Equation 4-5.

$$
d \mathrm{G}=d \mathrm{H}-\mathrm{T} d \mathrm{~S}
$$

The stability criteria of a system can be defined in terms of Gibbs free energy as:

- If $\Delta \mathrm{G}=0$ the reaction is in equilibrium.

- If $\Delta \mathrm{G}<0$ the reaction is spontaneous.

- If $\Delta \mathrm{G}>0$ the reaction is non-spontaneous.

The Gibbs free energy delivers a chemical reaction stability criteria based solely on system properties with a constant temperature and pressure [31]. 


\section{Nucleation and Growth}

During phase transformation a new phase with different chemical and physical characteristics to those of the parent phase is formed. Generally, a phase transformation starts with the formation of several particles of the new phase that gradually increase in size upon completion of the transformation. The transformation process has two different stages, nucleation and growth. During nucleation small particles or nuclei of the new phase begin to appear. Then, during the growth period nuclei tend to increase in size resulting in a partial or total disappearance of the parent phase [32].

\section{Homogeneous Nucleation}

In phase transformations a relevant thermodynamic parameter is the change in free energy $\Delta G$, where a transformation will happen spontaneously only if $\Delta \mathrm{G}<0$. Assume that individual nuclei are spherical with a radius $r$ as exemplified in Figure 4-1. Two contributions make the solidification transformation possible. The first contribution is the product of $\Delta \mathrm{G}_{\mathrm{v}}$ and the volume of the spherical nucleus [32]. $\Delta \mathrm{G}_{\mathrm{v}}$ is the volume free energy which is the difference between the solid and liquid phases. The second contribution takes into account the formation of the solid-liquid phase boundary during solidification. This term arises from the surface energy $\gamma$, multiplied by the surface area of the nucleus $\left(4 \pi r^{2}\right)$, both terms are being displayed on the right hand side of Equation 4-3.

$$
\Delta \mathrm{G}=4 / 3 \pi \mathrm{r}^{3} \Delta \mathrm{G}_{\mathrm{v}}+4 \pi \mathrm{r}^{2} \gamma
$$

The total free energy contributions along with the volume and surface terms are being plotted as a function of the nucleus radius $r$ in Figure 4-2. The curve corresponding to the first term $\left(4 / 3 \pi r^{3} \Delta G_{v}\right)$ is negative due to the free energy which allows the term to decrease with the third power of $r$. The plot of the second term which has a positive energy value, tends to increase with $r^{2}$. The plot of $\Delta \mathrm{G}$ which is the sum of the both terms, tends to initially increase, reach a 
maximum and then decrease. A solid particle starts to form when atoms in the liquid group bound together, here its free energy first increases [32]. At this point, the cluster acquires a size equal to the critical radius $r^{*}$, then growth will occur along with a decrease in free energy.

However, if a cluster forms with a radius smaller than $r^{*}$, it will shrink and ultimately disintegrate. Particles with a radius smaller than $r^{*}$ are known as embryo and the particles with radius greater than $r^{*}$ are referred as nucleus. The critical free energy $\Delta \mathrm{G}^{*}$ occurs at the critical radius $\left(r^{*}\right)$ which is at the maximum value of the curve (See Figure 4-2). $\Delta \mathrm{G}^{*}$ represents the activation free energy that is the energy required for the formation of a stable nucleus which can be considered an energy barrier to the nucleation process [32].

\section{Heterogeneous Nucleation}

Supercooling levels for homogenous nucleation reach the hundreds of degrees Celsius. The reason for such phenomena can be attributed to the activation energy for nucleation which is decreased when nuclei form on interfaces, since the surface free energy $(\gamma)$ is reduced. Nucleation tends to occur at surfaces and interfaces rather than other locations [32].

Consider the nucleation on a flat surface, over a solid particle from an existing liquid phase. In this case both liquid and solid phases wet the flat surface, spreading out and completely cover the surface (See Figure 4-3). Three interfacial energies are represented as vectors, existing on the two-phase boundary, the solid surface $\gamma_{\mathrm{SI}}$, solid-liquid $\gamma_{\mathrm{SL}}$, liquid-surface $\gamma_{\mathrm{IL}}$, and the wetting angle located between vectors $\gamma_{\mathrm{SI}}$ and $\gamma_{\mathrm{SL}}$. The relation between $\Delta \mathrm{G}^{*}$ het and $\Delta \mathrm{G}^{*}$ hom is given by Equation 4-4. Equation 4-5 displays the $S(\theta)$ factor, which is a function solely dependent on $\theta$ with values between 0 and 1 , influenced mainly by the shape of the nuclei $[32][33]$.

$$
\begin{gathered}
\Delta \mathrm{G}^{*}{ }_{\text {het }}=\Delta \mathrm{G}^{*}{ }_{\text {hom }} \mathrm{S}(\theta) \\
\mathrm{S}(\theta)=(2+\cos \theta)(1-\cos \theta)^{2} / 4
\end{gathered}
$$


Figure 4-4 displays the plot of $\Delta \mathrm{G}$ with respect to the nucleus' radius. It indicates the magnitude difference between the homogeneous and heterogeneous nucleation. A reduced $\Delta \mathrm{G}^{*}$ het on the critical radius indicates that a smaller energy will be the threshold $\Delta \mathrm{G}^{*}$ het during the nucleation process, allowing heterogeneous nucleation to occur more readily [32].

\section{Growth}

Particle growth in the transformation process starts when an embryo surpasses the critical radius $r^{*}$ and develops into a nucleus. The growth process will stop in areas where particles of the new phase are present, because in such areas the transformation already occurred. Particle growth develops by means of a long-range atomic diffusion, involving numerous steps including diffusion through the parent phase, across a phase boundary, and finally into the nucleus. The growth rate $\dot{G}$ is strictly dependent on the diffusion rate and temperature displayed in Equation 46. Where $Q$ is the activation energy, $C$ a pre-exponential constant factor, $k$ is the Boltzmann constant and $T$ is the temperature in degrees Kelvin [32].

$$
\dot{\mathrm{G}}=\mathrm{C} \exp (-Q / k T)
$$

Figure 4-5 shows a curve of the nucleation rate $\dot{N}$, where the total transformation rate is equal to the product of $\dot{G}$ and $\dot{N}$. The total transformation rate curve represents such product. The current phase growth analysis was developed for solidification but it is also applicable in scenarios solid-solid and solid-gas transformations [32]. The transformation rate and the time needed for the transformation to achieve a partial completion (50\% of reaction completion, $\mathrm{t}_{0.5}$ ) tend to be inversely proportional as displayed on Equation (4-7).

$$
\text { rate }=\left(1 / t_{0.5}\right)
$$

Different phenomena along the transformation process can be justified by the curves displayed in Figure 4-5. The new phase particle size strictly depends on the transformation 
temperature. Transformations that arise at temperatures close to the melting temperature, $T_{m}$, correspond to a low nucleation and a high growth rate. Hence, the subsequent microstructure will include a few but relatively large phase particle or coarse grains. On the other hand, for transformations at lower temperatures, the rates of nucleation tend to be high and with lower growth rates, producing several tiny particles (grains) [32].

\section{Phase Diagrams}

Phase diagrams are clear maps that give the relationships between phases in thermodynamic equilibrium in a system as a function of temperature, pressure, and composition [33]. In metallurgy, phase is generally referred to the different types of atomic bonding and arrangement of elements in a given material of a specific chemical composition. The three phases (solid, liquid, and gas) of a pure metal have the same chemical composition, but physically distinct in the atomic arrangement. The atomic bonding in solids develops in a repetitive arrangement (crystal structure). Generally, solid metals have their atoms arranged in a specific crystallographic structure. Although, some metals tend to vary their crystallographic structure as a function of temperature [34]. Alloying two or more elements usually affects the existence of phase changes. The stoichiometry of the mixture of two or more elements can induce the formation of different crystalline phases or chemical compounds. Phase diagrams provide a graphical representation of the phase changes occurring in a specific material and are also employed for development of new alloys for specific applications [34].

\section{Binary Systems}

Binary systems include the phases of formation between two elements at different alloy composition at multiple temperatures. Alloys containing metals of different crystalline structures will have complex phase diagrams. Figure 4-6 displays the phase diagram of nickel and copper, which is an isomorphous phase diagram due to the elements' miscibility onto one another in the 
solid state [34]. The diagram displays the phases present in all alloys that can be formed with nickel and copper metals at temperatures from 500 to $1,500^{\circ} \mathrm{C}$. The composition of the alloy is plotted on the horizontally, where the atomic percentage and weight percentage can be found at the top and bottom axes, respectively. The temperature is read vertically, where a centigrade and a Fahrenheit scale can be located on the left and right axes respectively. The upper and the lower curves run from the melting point of nickel $\left(1,452{ }^{\circ} \mathrm{C}\right)$ to the melting point of copper $\left(1,083{ }^{\circ} \mathrm{C}\right)$ [34]. The upper curve, namely the liquidus, displays for each alloy composition the specific temperature in which the alloy begins to solidify (freeze) upon cooling or in other words, when melting is completed upon heating. The solidus curve specifies the temperatures when melting starts upon heating or when solidification is completed upon cooling. The section above the liquidus curve is denoted by $L$ and every alloy will be at a liquid state at that region. Under the solidus curve all alloys will be in the solid phase. This region is labeled with $\alpha$ designated to the solid solution. At temperature gradients between the curves, both solid and liquid phase are present, thus it is labeled as $\alpha+L$ [34].

\section{Equilibrium}

The phase diagrams provide the phase relationships when equilibrium has been achieved. A state of equilibrium can be represented as heterogeneous equilibrium due to the coexistence of different states of matter. For two or more phases to achieve mutual equilibrium, it is essential that each phase is internally in a homogeneous state, thus each phase should be in the lowest free energy state possible. Hence, the chemical composition should be the same within the phase and the crystalline phases should be free of internal stresses [33]. Equilibrium can be understood as a state of a system where the phase characteristics remain constant over indefinite time periods. At equilibrium the free energy is a minimum [35]. 
During a phase transformation, an alloy stabilizes at an equilibrium state specified by the phase diagram in terms of phase, temperature and composition. Additionally, phase transformations necessitate a finite timeframe to achieve completion, where the speed in which it is accomplished is crucial for the microstructure development. When phase transformations are generated by changes in temperature, equilibrium can be conserved if cooling or heating are achieved at extremely slow rates [32].

\section{The Phase Rule}

The Gibbs phase rule states the maximum number of phases, $P$, which can coexist in a chemical system, plus the number of degrees of freedom, $F$, is equal to the sum of the components, $C$, of the system plus 2, as displayed by Equation 4-8 [34].

$$
\mathrm{P}+\mathrm{F}=\mathrm{C}+2
$$

The phases represented by $P$ are the homogeneous parts of a system (liquid, solid, and gas) that can be changed by external parameters including pressure and temperature. The degrees of freedom, $F$, are external conditions that shall be specified to ultimately define the equilibrium state of a system. The controllable conditions include temperature, pressure, and composition. The components, $C$, are the smallest number of substances of independent variable composition. For the scope of this research work the components will be evaluated solely for binary alloys, thus, the $C$ parameter will be equal to 2 . Moreover, in the case that the compound such as salt and water $\left(\mathrm{H}_{2} \mathrm{O}+\mathrm{NaCl}\right)$ the number of components should be two. Except if the mixture is exposed to temperature and pressure conditions where one or both compounds will decompose. Then, it will be necessary to include all four components (water, oxygen, sodium, and chlorine) into the $C$ variable [34]. 


\section{Laser Interaction}

A target material can be melted and vaporized by means of interactions with the radiation of a laser energy beam. Such interactions are highly dependent on the laser pulse duration, power density, and the peak spiking power [36]. Understanding the material laser interaction can be difficult since there exists instantaneous interactions of various complex physical processes. Figure 4-7 displays a block diagram reprensnting the microscopic power balance at an instant in time along the interaction of an incident laser beam with a metal surface [36]. It can be observed that only a a fraction of the incident laser energy will be absorbed by the material due to several losses along the process including reflection and scattering. The scattering encompasses heat loss due to material being evaporated and immediately ejected from the system along with a loss in reflection due to optical properties. The absorbed incident laser energy will be converted to thermal power. Hence, the temperature in a local point can be drastically raised and begin instant vaporization. Simultaneously, thermal diffusion starts as the temperature increases in the focused point. Ultimately, removal from the target material can develop as a consequence of vaporization, crater formation due to vapor expansion, and the formation of a molten layer of the target material [36].

\section{Metals}

The understanding of the laser absorption process is crucial in order to implement laser processing of metals. The heat absorption from the laser beam is strictly dependent on different properties from both, the laser source and the target metal. At short penetration depths in the order of tens of nanometers is common in target metals for infrared and visible light. Absorption tends to be a surface phenomenon and strictly depends on the surface properties and finishing of the target metal. The surface roughness, texture, along with the presence of an oxide layer tend to influence the laser interaction [37]. 
When light strikes a target surface the incoming laser energy will be reflected, transmitted or absorbed by the material. The bi-directional reflection function which is the radiative property of interest is expressed in terms of incident power and scattered power. The bidirectional reflection function (BDRF) is expressed as $\pi$ times the ratio of the reflected radiant power per unit solid angle per unit projected area to the incident radiant power. Where $\Phi$ is the radiant power and $\Omega$ is the solid angle where the subscripts $o$ and $s$ specify incidence or scattering, respectively [38]. Equation 4-9 displays the BDRF function.

$$
\rho^{\prime \prime}\left(\theta_{\mathrm{O}}, \theta_{\mathrm{S}}\right)=\frac{\frac{\pi}{\cos \theta_{S}}\left(\frac{d \theta_{S}}{d \Omega_{\mathrm{S}}}\right)}{\frac{d \theta_{\mathrm{O}}}{d \Omega_{\mathrm{O}}}}
$$

Studies have shown that an increase in roughness $\sigma / \tau$ increases the absorption of the incoming laser energy. In the aforementioned ratio, $\sigma$ is the root-mean square roughness height and $\tau$ the surface correlation length [37]. Figure 4-8 displays the absorption for normal incident light shown as a function of roughness for different metals. The trend displays that roughening a surface of a high reflectivity metal can increase its absorption capability in Nd:YAG laser light by several hundred percent [37]. In normal incident laser light, the absorption of the target metal tends to increase with the surface roughness after a certain threshold has been surpassed. This is due to the onset of numerous scattering events within the target surface.

\section{Ceramics}

As in the case of metals, a suitable sintering parameter shall be implemented due to the complex nature of laser material interaction with ceramic targets. An effective preparation heavily depends on a fully or partially melting of solid powder granules [39]. Generally, the molten compound wets the solid powder granules and generates a viscous flow to increase the formation of a molten pool. When the laser beam strikes the powder granules, it experiences 
various scatters before it is completely absorbed by the target. The resulting heat formation on the target is usually inhomogeneous due to the unevenness in the laser energy distribution.

Hence, the temperature distribution along the process will tend to be uneven during the interaction time, and as a result, the cooling will also be inhomogeneous. Ceramic compounds tend to have high melting points when compared to metals. Consequently, it can be challenging to melt and tend to require high energy inputs [39].

Laser synthesis is a pressure-less process where the elimination of porosities is achieved by capillary forces. Hence, the compaction of the sample powder is crucial to obtain a high product density. Additionally, the surface smoothness of the sintered compounds can be enhanced when submicron size powders are being employed [39]. Additional parameters including the powder granule shape, size, and homogeneity can influence the laser synthesized product. Therefore, it is advised to consider the aforementioned factors while analyzing the laser material interaction.

In addition, due to the extreme heating and cooling rates generated during the laser synthesis, the formation of a single phase or a collection of phases can occur at any stage of the process. Phase transitions can be influenced and unwanted phases can be preserved in the product compound at the end of the process [39]. A successful laser synthesis of ceramic compounds will highly depend on a better understanding of the laser material interaction. Thus, it is crucial to incorporate an enhanced control of structural heterogeneities and thermal stresses in order to prevent defects to successfully produce ceramic compounds.

\section{Phase Diagram Analyses - Uranium and Surrogate Materials}

\section{Ce-Si}

The cerium-silicon binary phase diagram is characterized by the presence of six intermediate phases including $\mathrm{Ce}_{5} \mathrm{Si}_{3}, \mathrm{Ce}_{3} \mathrm{Si}_{2}, \mathrm{Ce}_{5} \mathrm{Si}_{4}, \mathrm{CeSi}, \mathrm{Ce}_{3} \mathrm{Si}_{5}$ and $\mathrm{CeSi}_{2}$ [40]. According to 
the $\mathrm{Ce}-\mathrm{Si}$ binary phase diagram, $\mathrm{Ce}_{3} \mathrm{Si}_{2}$, is formed when the stoichiometric proportions of cerium (At. 60\%) and silicon (At. 40\%) interact at $1,390{ }^{\circ} \mathrm{C}$ where congruent melting leads to the reaction $\mathrm{L} \leftrightarrow \mathrm{Ce}_{3} \mathrm{Si}_{2}$. A eutectic point exists at $1,270^{\circ} \mathrm{C}$ and $38.5 \%$ at. silicon, leading to the reaction $\mathrm{L} \leftrightarrow \mathrm{Ce}_{5} \mathrm{Si}_{3}+\mathrm{Ce}_{3} \mathrm{Si}_{2}$ that can induce the formation of the secondary phase $\mathrm{Ce}_{5} \mathrm{Si}_{3}$ in addition to $\mathrm{Ce}_{3} \mathrm{Si}_{2}$. While employing a laser source, the melting temperature difference between cerium $\left(798^{\circ} \mathrm{C}\right)$ and silicon $\left(1,414^{\circ} \mathrm{C}\right)$ can led to the formation of a hypo-stoichiometric cerium compound. The formation can be attributed to the uneven laser heat distribution along with the lower melting point of cerium which can generate immediate vaporization, reducing the amount of cerium present in the reaction, thus, forming a silicon rich compound.

\section{Zr-Si}

The phases in a state of equilibrium in the zirconium-silicon system include: $\mathrm{ZrSi}_{2}, \beta$ $\mathrm{ZrSi}, \alpha-\mathrm{ZrSi}, \beta-\mathrm{Zr}_{5} \mathrm{Si}_{4}, \alpha-\mathrm{Zr}_{5} \mathrm{Si}_{4}, \mathrm{Zr}_{3} \mathrm{Si}_{2}, \mathrm{Zr}_{5} \mathrm{Si}_{3} ; \mathrm{Zr}_{2} \mathrm{Si}, \mathrm{Zr}_{3} \mathrm{Si}, \beta-\mathrm{Zr}$ and $\alpha-\mathrm{Zr}$. The surrogate material selected for this research work is $\mathrm{Zr}_{3} \mathrm{Si}_{2}$ which can be formed when stoichiometric ratios of zirconium (At. 60\%) and silicon (At. 40\%) interact at a temperature of approximately 2,215 ${ }^{\circ} \mathrm{C}$ as advised on the Zr-Si binary phase diagram displayed on Figure 4-10 [41].

Based on the $\mathrm{Zr}-\mathrm{Si}$ binary phase diagram, $\mathrm{Zr}_{3} \mathrm{Si}_{2}$ is formed by a peritectic reaction, where a solid phase and a liquid phase will combine and form a new solid phase at specific temperature and composition. An advantage of zirconium and silicon is that both have relatively close melting points $\left(\mathrm{Zr}: 1,855^{\circ} \mathrm{C}-\mathrm{Si}: 1,414{ }^{\circ} \mathrm{C}\right)$ when compared to cerium and silicon, minimizing the risk of material vaporization during the laser material interaction.

\section{Hf-Si}

The phases found in the hafnium-silicon binary phase diagram include $\beta$-Hf, $\alpha-\mathrm{Hf}, \mathrm{Hf}_{2} \mathrm{Si}$, $\mathrm{Hf}_{3} \mathrm{Si}_{2}, \mathrm{Hf}_{5} \mathrm{Si}_{4}, \mathrm{HfSi}, \mathrm{HfSi}_{2}$, and $\mathrm{Si}$. The compound of interest, $\mathrm{Hf}_{3} \mathrm{Si}_{2}$, forms when stoichiometric quantities of hafnium (At. 60\%) and silicon (At. 40\%) undergo a congruent reaction at 
approximate temperatures of $2,480{ }^{\circ} \mathrm{C}$ as shown in the $\mathrm{Hf}-\mathrm{Si}$ phase diagram displayed on Figure 4-11 [42].

According to the Hf-Si binary phase diagram, the $\mathrm{Hf}_{3} \mathrm{Si}_{2}$ phase displays congruent melting, where a binary compound melts at a certain concentration into a liquid of its own composition. The melting temperature difference between hafnium and silicon is fairly large (Hf: $2,226.85^{\circ} \mathrm{C}-\mathrm{Si}: 1,414^{\circ} \mathrm{C}$ ). Thus, during the laser synthesis silicon can melt faster than hafnium and even vaporize, leading to the peritectic reaction $\mathrm{L}+\mathrm{Hf}_{3} \mathrm{Si}_{2} \leftrightarrow \mathrm{Hf}_{2} \mathrm{Si}$ along with the eutectic reaction $\mathrm{L} \leftrightarrow \beta-\mathrm{Hf}+\mathrm{Hf}_{2} \mathrm{Si}$ that may aid in the formation of a hafnium rich phase, $\mathrm{Hf}_{2} \mathrm{Si}$.

\section{U-Si}

The binary phases encountered on the uranium-silicon phase diagram were: $\mathrm{USi}_{3}, \mathrm{USi}_{1.88}$, $\mathrm{USi}_{2}, \mathrm{U}_{3} \mathrm{Si}_{5}, \mathrm{U}_{3} \mathrm{Si}_{2}, \mathrm{U}_{3} \mathrm{Si}, \alpha-\mathrm{U}, \beta-\mathrm{U}, \gamma-\mathrm{U}$. According to the phase diagram in Figure 4-12, the formation of $\mathrm{U}_{3} \mathrm{Si}_{2}$ occurs when stoichiometric quantities of uranium (At. 60\%) and silicon (At. $40 \%$ ) are combined at a temperature of approximate $1,665^{\circ} \mathrm{C}$ [17]. The formation of $\mathrm{U}_{3} \mathrm{Si}_{2}$ occurs by means of congruent melting, allowing coexistence of the compound in both a solid and a liquid phase. The melting temperature difference for uranium $\left(1,135^{\circ} \mathrm{C}\right)$ and silicon $\left(1,414{ }^{\circ} \mathrm{C}\right)$ is fairly small when compared to the previous surrogates, and can generate a homogeneous melting during the laser synthesis allowing the formation of the desired compound.

Additionally, a eutectic point exists at $1,540^{\circ} \mathrm{C}$ over the $\mathrm{U}_{3} \mathrm{Si}_{2}$ region. The microstructure of the resulting solid at the eutectic point may consist of alternating layers of the adjacent phases $\mathrm{U}_{3} \mathrm{Si}$ and $\mathrm{U}_{3} \mathrm{Si}_{2}$ that form simultaneously during the transformation. The formation of such pattern can be attributed to the redistribution of atoms accomplished by atomic diffusion, the resulting microstructure is also known as lamellar structure [33]. 


\section{Selection of Surrogate Materials}

\section{Formation of Alloys}

The formation of alloys generally depend on certain parameters including the manner in which the alloying materials were mixed with each other, the proportion of the different alloying material and the temperature at which the alloys are cooled. When an alloy is on its liquid state, the atomic distribution along the liquid is random. When solidification occurs, a number of different possibilities for the atomic arrangements can occur [35]. Crystals formed with elements having a distinct crystal lattice which is different from the crystal lattice of the component elements are referred to intermediate phases. Intermediate phases usually have a bond that is dependent upon their components and generally determines the properties of the crystal. The atomic arrangement in the lattices can be disordered, partially ordered or entirely ordered.

Additionally, intermediate phases are identified as crystals whose composition can differ in a wide or narrow range in concentration [35]. The changing composition can be justified by the presence of interstitial atoms in the lattice or deficiency (absence) of atoms in the crystal lattice. A constant cooling temperature of crystallization can be achieved if the cooling is preserved in equilibrium with the surroundings. The quicker the cooling, the lower the temperature where the crystal formation initiates. Thus, it is recommended to employ slow cooling rates to allow time for the arrangement of the atoms to be completed [35].

\section{Surrogate Selection}

This research work has initially considered cerium as the main surrogate material for uranium fuel. However, different properties of alternate surrogate materials have been evaluated in order to incorporate them into experimental design of the additive manufacturing process. In addition to cerium, other surrogate materials employed for nuclear fuel surrogates were evaluated including zirconium and hafnium. Zirconium has comparable chemical and 
crystallographic properties to those of uranium. In the form of $\mathrm{Zr}_{3} \mathrm{Si}_{2}$, the compound is isostructural with $\mathrm{U}_{3} \mathrm{Si}_{2}$ displaying a tetragonal crystal structure. Additionally, the $\mathrm{Zr}$-Si binary phase diagram has many similarities with the U-Si phase diagram, including numerous intermediate phases and multiple eutectic points.

Prior research studies show the feasibility of employing $\mathrm{Zr}_{3} \mathrm{Si}_{2}$ as a neutron reflector in the core of future gas cooled nuclear reactors [8] and $\mathrm{ZrO}_{2}$ has been employed as a surrogate material for uranium fuel in TRISO coated fuel [45]. Hafnium which has similar characteristics to those of zirconium [46] including crystal structure, enthalpy of formation, heat capacity, and thermal expansion coefficient was also evaluated to be incorporated in the experimental matrix (Table 4-1). In previous work, hafnium has been successfully employed as a uranium surrogate to simulate fuel debris from the Fukishima Daiichi nuclear event to develop a dust collection process [46][47]. Due to the aforementioned similarities with zirconium, including the resemblance with the $\mathrm{U}-\mathrm{Si}$ binary phase diagram, and the isostructural nature of $\mathrm{Hf}_{3} \mathrm{Si}_{2}$ with $\mathrm{U}_{3} \mathrm{Si}_{2}$, hafnium was down selected as a surrogate material for the experimental procedures for the additive manufacturing process.

Cerium is a non-radioactive element with similar crystallographic properties to those of uranium. In research studies, $\mathrm{CeO}_{2}$ has been successfully employed as a surrogate material for accident tolerant fuel, displaying high radiation stability and an irradiation performance similar to that of $\mathrm{UO}_{2}$ and $\mathrm{PuO}_{2} \cdot \mathrm{Ce}_{3} \mathrm{Si}_{2}$ is isostructural with $\mathrm{U}_{3} \mathrm{Si}_{2}$ displaying a tetragonal crystal structure and has already employed as a $\mathrm{U}_{3} \mathrm{Si}_{2}$ surrogate for oxidation and corrosion tests [6]. Additionally, $\mathrm{Ce}_{3} \mathrm{Si}_{2}$ melts congruently with $\mathrm{U}_{3} \mathrm{Si}_{2}$ and both binary phase diagrams (U-Si and CeSi) display various similarities including multiple eutectic points and numerous intermediate phases. Due to the aforementioned properties and a similarities between $\mathrm{Ce}_{3} \mathrm{Si}_{2}$ and $\mathrm{U}_{3} \mathrm{Si}_{2}$, 
cerium has been down selected to be included in the experimental matrix to undergo a laser synthesis [17].

In order to form the desired compound $\left(\mathrm{Ce}_{3} \mathrm{Si}_{2}, \mathrm{Zr}_{3} \mathrm{Si}_{2}, \mathrm{Hf}_{3} \mathrm{Si}_{2}\right)$ the stoichiometric ratio of 60 At. $\%$ metal and 40 At.\% silicon should be present during the laser material interaction. Nonetheless, due to an inhomogeneous heat distribution, some unreacted silicon or metal will allow the formation of metal rich and/or silicon rich phases, generally the neighboring phases (left and right of the desired phase) on the binary phase diagram. Due to the inherited thermodynamic properties, each surrogate compound $\left(\mathrm{Ce}_{3} \mathrm{Si}_{2}, \mathrm{Zr}_{3} \mathrm{Si}_{2}, \mathrm{Hf}_{3} \mathrm{Si}_{2}\right)$ displays a specific stoichiometric interval that may allow its formation. Based on other works, the reported intervals are 60-62.5 At\% cerium for $\mathrm{Ce}_{3} \mathrm{Si}_{2}$ [40], 55-60 At.\% zirconium for $\mathrm{Zr}_{3} \mathrm{Si}_{2}$ [41], and 60-66.5 At.\% hafnium for $\mathrm{Hf}_{3} \mathrm{Si}_{2}$ [42].

\section{Thermodynamic Simulations}

Thermodynamic simulations were performed with the software package ThermoCalc®. ThermoCalc $®$ is a software package developed for computational materials engineering. It is widely used in applied research in applications for designing new alloys, optimizing processing conditions, failure analysis, modeling, and simulating the evolution of microstructure.

Simulations were performed on the formation reaction of both silicide fuel and silicide surrogate fuel. The reaction evaluated was $3 \mathrm{M}+2 \mathrm{Si} \rightarrow \mathrm{M}_{3} \mathrm{Si}_{2}$ where $\mathrm{M}$ stands for the different metals evaluated. The materials included on the simulation were uranium along with zirconium and hafnium as surrogate materials (cerium was omitted due to database limitations). The $\Delta \mathrm{G}$ of the reaction was evaluated at different pressures $(100,000 \mathrm{~Pa}, 01 . \mathrm{Pa}$, and $0.0001 \mathrm{~Pa})$ in order to evaluate the pressure effect of a high vacuum at different temperature gradients after the lasermaterial interaction, as that is when nucleation and growth of the desired phase begin to occur. 
The simulation results on the $\Delta \mathrm{G}$ as a function of temperature for $\mathrm{Zr}_{3} \mathrm{Si}_{2}, \mathrm{Hf}_{3} \mathrm{Si}_{2}$, and $\mathrm{U}_{3} \mathrm{Si}_{2}$ are displayed on Figure 4-13, 4-14, and 4-15, respectively. From the simulation results, it can be observed that $\Delta \mathrm{G}$ is conservative among the different pressures evaluated $(100,000 \mathrm{~Pa}$, 01. Pa, and 0.0001 Pa). The pressure effect provided a negligible effect on phase changes and overall compound formation. The temperatures from interest are beyond $1,000^{\circ} \mathrm{C}$ where the melting of the elements and the formation of the desired compounds occurs. At temperature gradients between 1,000 and $2,200{ }^{\circ} \mathrm{C}$ the $\Delta \mathrm{G}$ remained less than zero, indicating that a spontaneous reaction may occur if the pressure and temperature remain constant. The Gibbs free energy delivers a chemical reaction stability criteria based solely on system properties with a constant temperature and pressure [32]. The simulation results from ThermoCalc $®$ proved that the effect of pressure generated by a high vacuum during the experimental stage will not affect the formation of the desired compounds in this phase of experimentation.

\section{Conclusion}

The down selection of the surrogate materials was based on the crystallographic structure of the compounds, all preserving a tetragonal crystal structure $\left(\mathrm{U}_{3} \mathrm{Si}_{2}, \mathrm{Ce}_{3} \mathrm{Si}_{2}, \mathrm{Zr}_{3} \mathrm{Si}_{2}\right.$, and $\left.\mathrm{Hf}_{3} \mathrm{Si}_{2}\right)$ along with the similarities encountered on their respective phase diagrams. The relevant similarities were multiple eutectic points along with different phases of formation of the binary compounds. Objective 1 aimed to analyze the binary phase diagrams of selected surrogate materials, such analyses were crucial, as they allowed to determine the exact proportions of the sample powders along with the threshold temperatures in order to form surrogate compounds with the desired stoichiometry. Additionally, the binary phase diagrams allowed to predict secondary phases that can potentially appear due to an uneven heat distribution from the laser sintering source. A high power laser output is recommended during the laser synthesis due to a high melting point in ceramic compounds. The powder packing density (which is a function of 
the powder distribution) of the sample powders is crucial to obtain high product densities.

Additionally, the surface smoothness of the sintered compounds can be enhanced when submicron size powders are being employed. However, this should be considered in conjunction with the reactivity nature of the powder and handling aspects for safety reasons. A successful laser synthesis of ceramic compounds will highly depend on a better understanding of the laser material interaction, which is evaluated in the next chapter (Chapter 5). 
Table 4-1. Property list of thermodynamic and mechanical properties of selected compounds.

\begin{tabular}{|c|c|c|c|c|c|c|c|c|c|}
\hline & $\mathrm{U}$ & $\mathrm{UF}_{4}$ & $\mathrm{U}_{3} \mathrm{Si}_{2}$ & $\mathrm{Ce}$ & $\mathrm{CeF}_{4}$ & $\mathrm{Ce}_{3} \mathrm{Si}_{2}$ & Hf & $\mathrm{HfF}_{4}$ & $\mathrm{Hf}_{3} \mathrm{Si}_{2}$ \\
\hline Atomic Number & 92 & - & - & 58 & - & - & 72 & - & - \\
\hline Atomic Mass (amu) & 238.02 & 314 & 770.24 & 140.11 & 215.89 & 476.5 & 178.49 & 254.48 & 591.64 \\
\hline Melting Point $\left({ }^{\circ} \mathrm{C}\right)$ & $1,132.20$ & 960 & 1,665 & 798 & 650 & 1,335 & $2,226.85$ & 1,045 & 2,480 \\
\hline Boiling Point $\left({ }^{\circ} \mathrm{C}\right)$ & $4,131 \mathrm{C}$ & $1,417 \mathrm{C}$ & - & 3,443 & - & - & $4,602.85$ & 970 & - \\
\hline $\begin{array}{l}\text { Heat of Fusion } \\
(\mathrm{KJ} / \mathrm{mol})\end{array}$ & 9.14 & 42.7 & - & 5.46 & - & - & 27.2 & - & - \\
\hline Density $\left(\mathrm{g} / \mathrm{cm}^{3}\right)$ & 19.1 & 6.7 & 12.2 & 6.77 & 4.77 & 5.96 & 13.31 & 7.1 & 10.95 \\
\hline Crystal Structure & Orthorombic & $\begin{array}{l}\text { Monoclinic } \\
\mathrm{mS} 60\end{array}$ & Tetragonal & $\begin{array}{l}\text { Double } \\
\text { Hexagonal } \\
\text { Closed pack } \\
\text { (Beta Ce) }\end{array}$ & Monoclinic & Tetragonal & $\begin{array}{l}\text { Hexagonal } \\
\text { Closed } \\
\text { Packed }\end{array}$ & $\begin{array}{l}\text { Monoclinic } \\
\text { ms60 }\end{array}$ & Tetragonal \\
\hline Space Group & $\mathrm{Cmcm}$ & $\mathrm{C} 2 / \mathrm{c}$ & $\mathrm{P} 4 / \mathrm{mbm}$ & $\mathrm{P} 63 / \mathrm{mmc}$ & $\mathrm{C} 2 / \mathrm{c}$ & $\mathrm{P} 4 / \mathrm{mbm}$ & $\mathrm{P} 63 / \mathrm{mmc}$ & $\mathrm{C} 2 / \mathrm{c}$ & $\mathrm{P} 4 / \mathrm{mbm}$ \\
\hline Pearson Symbol & oC4 & mS60 & $\mathrm{tP} 10$ & $\mathrm{mS} 60$ & $\mathrm{mS} 60$ & $\mathrm{tP} 10$ & $\mathrm{hP} 2$ & $\mathrm{mS} 60$ & $\mathrm{tP} 10$ \\
\hline $\begin{array}{l}\text { Entropy @25 ㄷ } \\
(\mathrm{J} / \mathrm{mol} \mathrm{K})\end{array}$ & 50.2 & 151.67 & - & 72 & - & - & 43.56 & 113 & - \\
\hline $\begin{array}{l}\text { Enthalpy of Formation } \\
\text { @ } 25{ }^{\circ} \mathrm{C}(\mathrm{KJ} / \mathrm{mol}) \\
\text { Gibbs Free Energy of }\end{array}$ & 0 & $-1,920$ & -33.86 & 0 & $-1,899$ & -60.9 & 0 & $-1,930.5$ & -400 \\
\hline $\begin{array}{l}\text { Formation @ } 25{ }^{\circ} \mathrm{C} \\
(\mathrm{KJ} / \mathrm{mol})\end{array}$ & 0 & $-1,829$ & $-180,121$ & 0 & - & -11.1 & 0 & $-1,830.5$ & - \\
\hline $\begin{array}{l}\text { Heat Capacity } \\
(\mathrm{J} / \mathrm{mol} \mathrm{K})\end{array}$ & 27.665 & 116 & 150 & 26.94 & - & - & 25.64 & 88 & - \\
\hline $\begin{array}{l}\text { Thermal Expansion } \\
\text { Coefficient }\left(\times 10^{-6} \mathrm{~K}^{-1}\right)\end{array}$ & 13.9 (RT) & - & 16.1 & $6.6(\mathrm{RT})$ & - & - & $5.9(\mathrm{RT})$ & - & - \\
\hline $\begin{array}{l}\text { Thermal Conductivity } \\
\text { (RT) (W/m K) }\end{array}$ & 27.5 & 1.96 & 15 & 11.3 & - & - & 23 & - & - \\
\hline $\begin{array}{l}\text { Specific Heat } \\
(\mathrm{KJ} / \mathrm{Kg} \mathrm{K})\end{array}$ & 0.12 & - & 0.187 & 0.19 & - & - & 0.14 & - & - \\
\hline
\end{tabular}

[14][16][44][48][49][50][51][52][53][54][55] 
Table 4-1. Continued.

\begin{tabular}{|c|c|c|c|c|c|c|}
\hline & $\mathrm{Zr}$ & $\mathrm{ZrF}_{4}$ & $\mathrm{Zr}_{3} \mathrm{Si}_{2}$ & $\mathrm{Si}$ & $\mathrm{La}$ & $\mathrm{Al}$ \\
\hline Atomic Number & 40 & - & - & 14 & 57 & 13 \\
\hline Atomic Mass (amu) & 91.22 & 167 & 329.84 & 28.08 & 138.9 & 26.98 \\
\hline Melting Point $\left({ }^{\circ} \mathrm{C}\right)$ & 1,855 & 910 & 2,215 & 1,414 & 920 & 660.32 \\
\hline Boiling Point $\left({ }^{\circ} \mathrm{C}\right)$ & 4,377 & - & - & 3,265 & 3,464 & 2,470 \\
\hline $\begin{array}{l}\text { Heat of Fusion } \\
(\mathrm{KJ} / \mathrm{mol})\end{array}$ & 14 & -64.22 & - & 50.21 & 6.2 & 10.71 \\
\hline Density $\left(\mathrm{g} / \mathrm{cm}^{3}\right)$ & 6.52 & 4.43 & 5.88 & 2.329 & 6.162 & 2.7 \\
\hline Crystal Structure & $\begin{array}{l}\text { Hexagonal } \\
\text { Closed } \\
\text { Packed }\end{array}$ & Monoclinic & Tetragonal & $\begin{array}{l}\text { Face centered } \\
\text { diamond cubic }\end{array}$ & $\begin{array}{l}\text { Double } \\
\text { Hexagonal } \\
\text { Close-Pack }\end{array}$ & $\begin{array}{l}\text { Face } \\
\text { centered } \\
\text { cubic }\end{array}$ \\
\hline Space Group & $\mathrm{P} 63 / \mathrm{mmc}$ & $\mathrm{C} 2 / \mathrm{c}$ & $\mathrm{P} 4 / \mathrm{mbm}$ & $\mathrm{Fd}-3 \mathrm{~m}$ & $\mathrm{P} 63 / \mathrm{mmc}$ & Fm-3m \\
\hline Pearson Symbol & $\mathrm{hP} 2$ & $\mathrm{mS} 60$ & $\mathrm{tP} 10$ & cI16 & $\mathrm{hP} 4$ & $\mathrm{cF} 4$ \\
\hline $\begin{array}{l}\text { Entropy @ } 25{ }^{\circ} \mathrm{C} \\
(\mathrm{J} / \mathrm{mol} \mathrm{K})\end{array}$ & 38.99 & 105 & - & 18.81 & 569 & 28.35 \\
\hline $\begin{array}{l}\text { Enthalpy of Formation } \\
\text { @ } 25{ }^{\circ} \mathrm{C}(\mathrm{KJ} / \mathrm{mol}) \\
\text { Gibbs Free Energy of }\end{array}$ & 0 & $-1,911.3$ & 384.56 & 0 & 0 & 0 \\
\hline $\begin{array}{l}\text { Formation @ } 25{ }^{\circ} \mathrm{C} \\
(\mathrm{KJ} / \mathrm{mol})\end{array}$ & 0 & $-1,810$ & & 0 & 0 & 0 \\
\hline $\begin{array}{l}\text { Heat Capacity } \\
(\mathrm{J} / \mathrm{mol} \mathrm{K})\end{array}$ & 25.37 & 102.8 & 118.74 & 19.789 & 27.11 & 24.20 \\
\hline $\begin{array}{l}\text { Thermal Expansion } \\
\text { Coefficient }\left(\times 10^{-6} \mathrm{~K}^{-1}\right)\end{array}$ & 5.7(RT) & - & 8.11 & $2.6(\mathrm{RT})$ & $12.1(\mathrm{RT})$ & $23.1(\mathrm{RT})$ \\
\hline $\begin{array}{l}\text { Thermal Conductivity } \\
\text { (RT) }(\mathrm{W} / \mathrm{m} \mathrm{K})\end{array}$ & 22.6 & - & - & 149 & 13.4 & 237 \\
\hline $\begin{array}{l}\text { Specific Heat } \\
(\mathrm{KJ} / \mathrm{Kg} \mathrm{K})\end{array}$ & 0.27 & - & - & 0.71 & 0.19 & 0.9 \\
\hline
\end{tabular}

[56][57][58][59][60][61][62][63][64][65][66] 


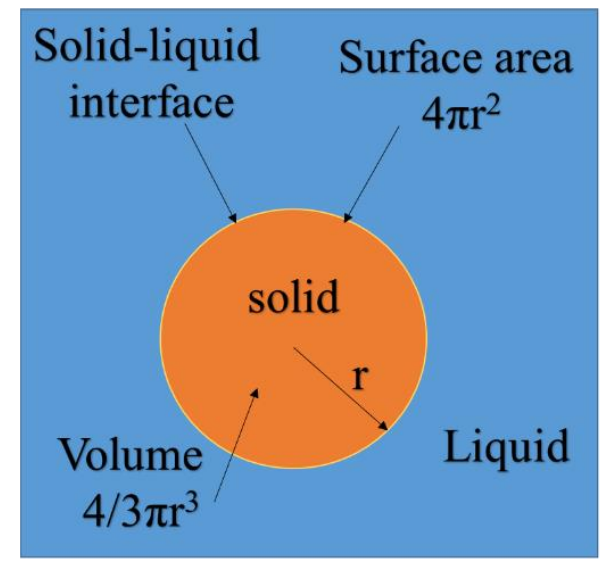

Figure 4-1. Diagram displaying the nucleation of a spherical solid particle in a liquid.

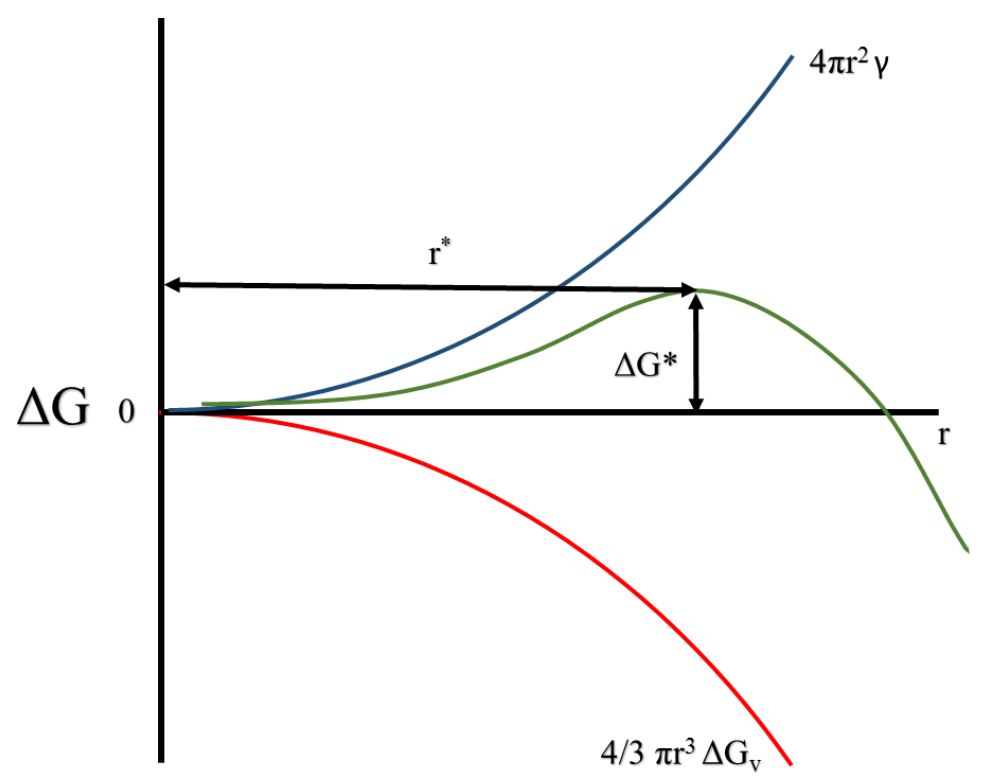

Figure 4-2. Plot of free energy versus embryo/nucleus radius, displaying the critical free energy change $G^{*}$ and the critical nucleus radius $r *$. 


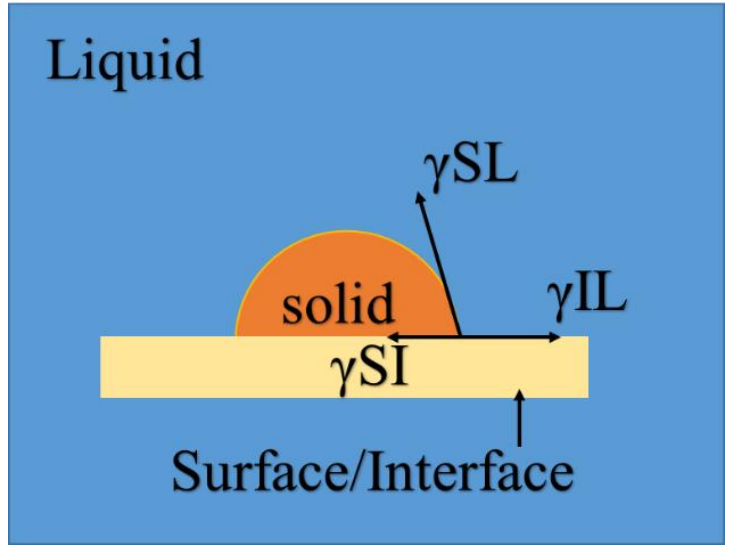

Figure 4-3. Heterogeneous nucleation of a solid from a liquid.

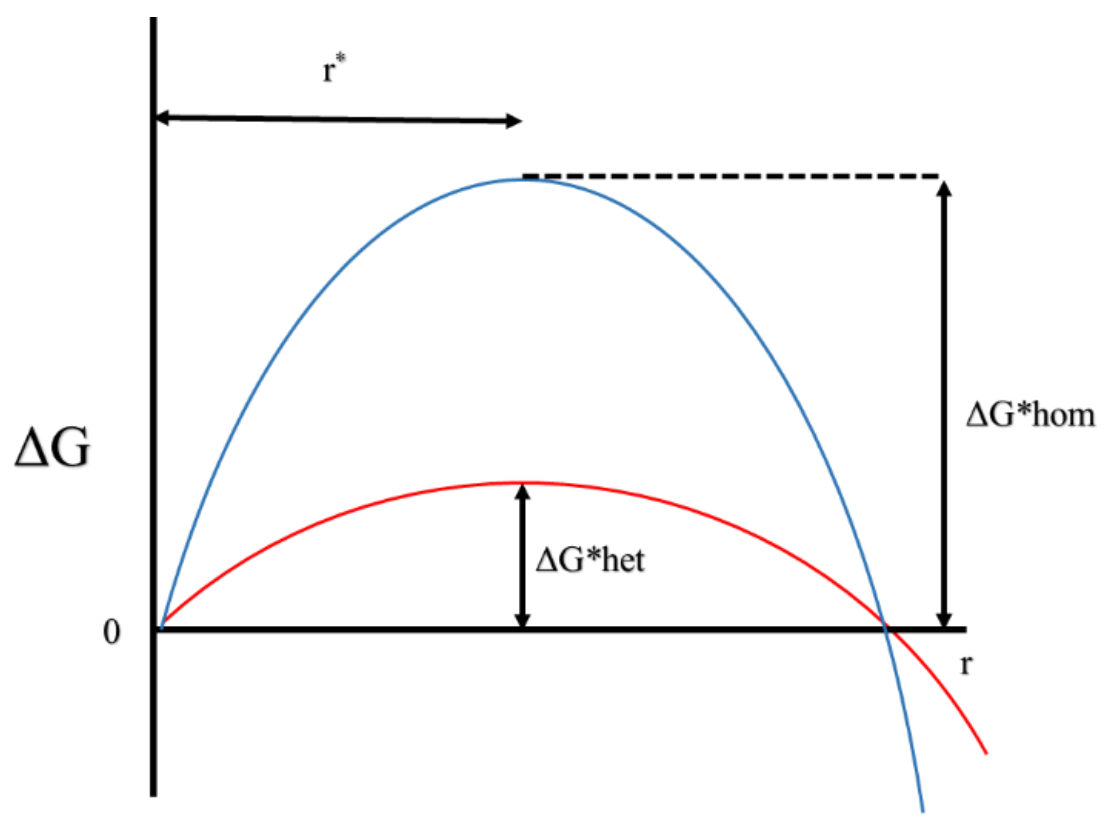

Figure 4-4. Plot representing curves for homogeneous and heterogeneous nucleation. The critical energies and critical radii are displayed. 


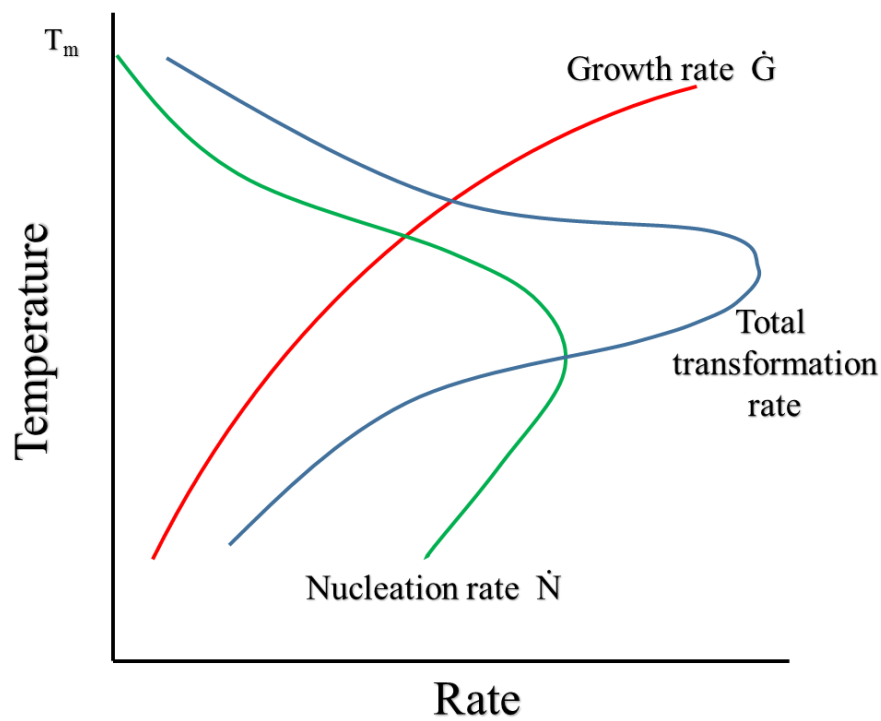

Figure 4-5. Plot displaying nucleation rate $(\dot{\mathrm{N}})$, growth rate $(\dot{\mathrm{G}})$ and total transformation versus temperature.

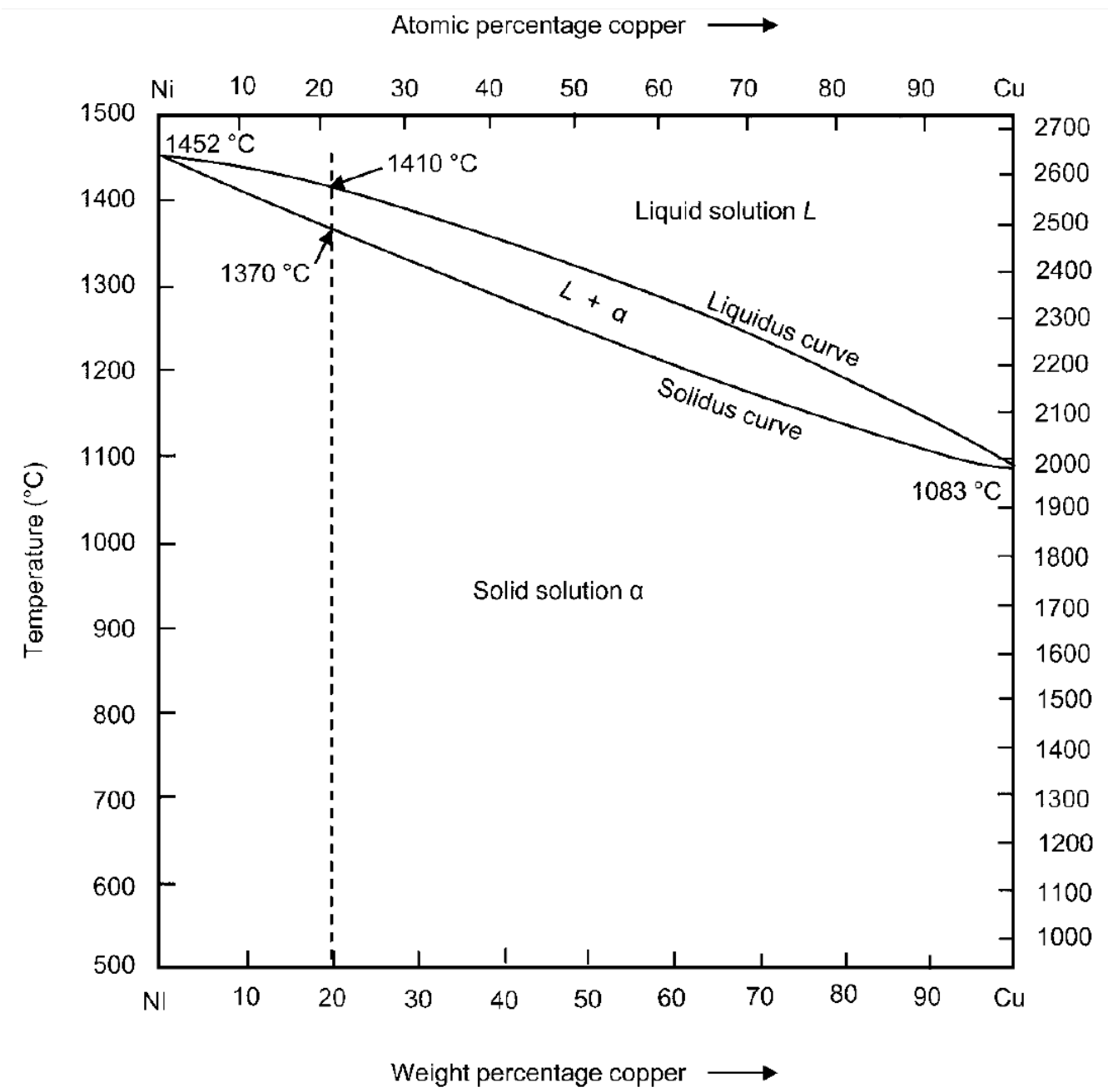

Figure 4-6. Binary phase diagram of copper and nickel [34]. 


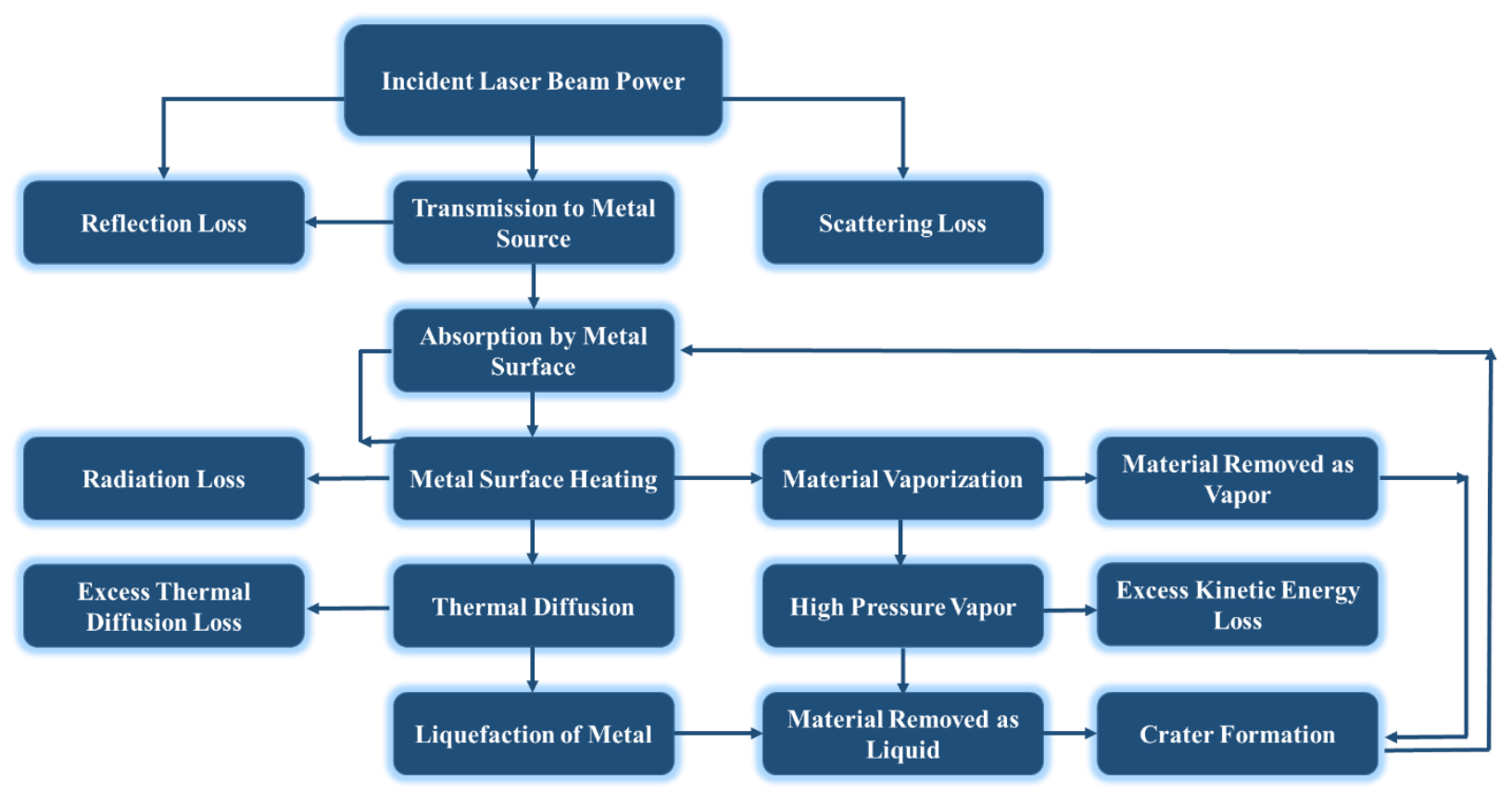

Figure 4-7. Block diagram of material removal process by means of laser energy interaction.

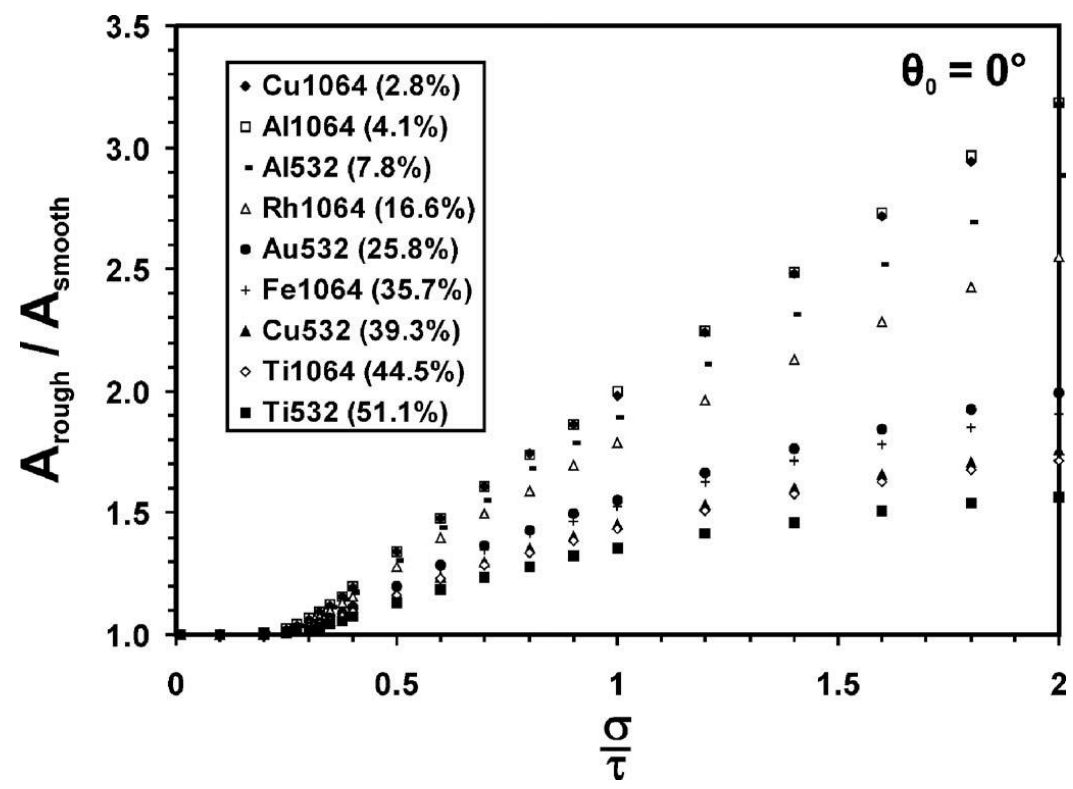

Figure 4-8. Ratios of the absorption of rough and smooth surfaces for normally incident light shown as a function of slope roughness for selected metals [37]. 


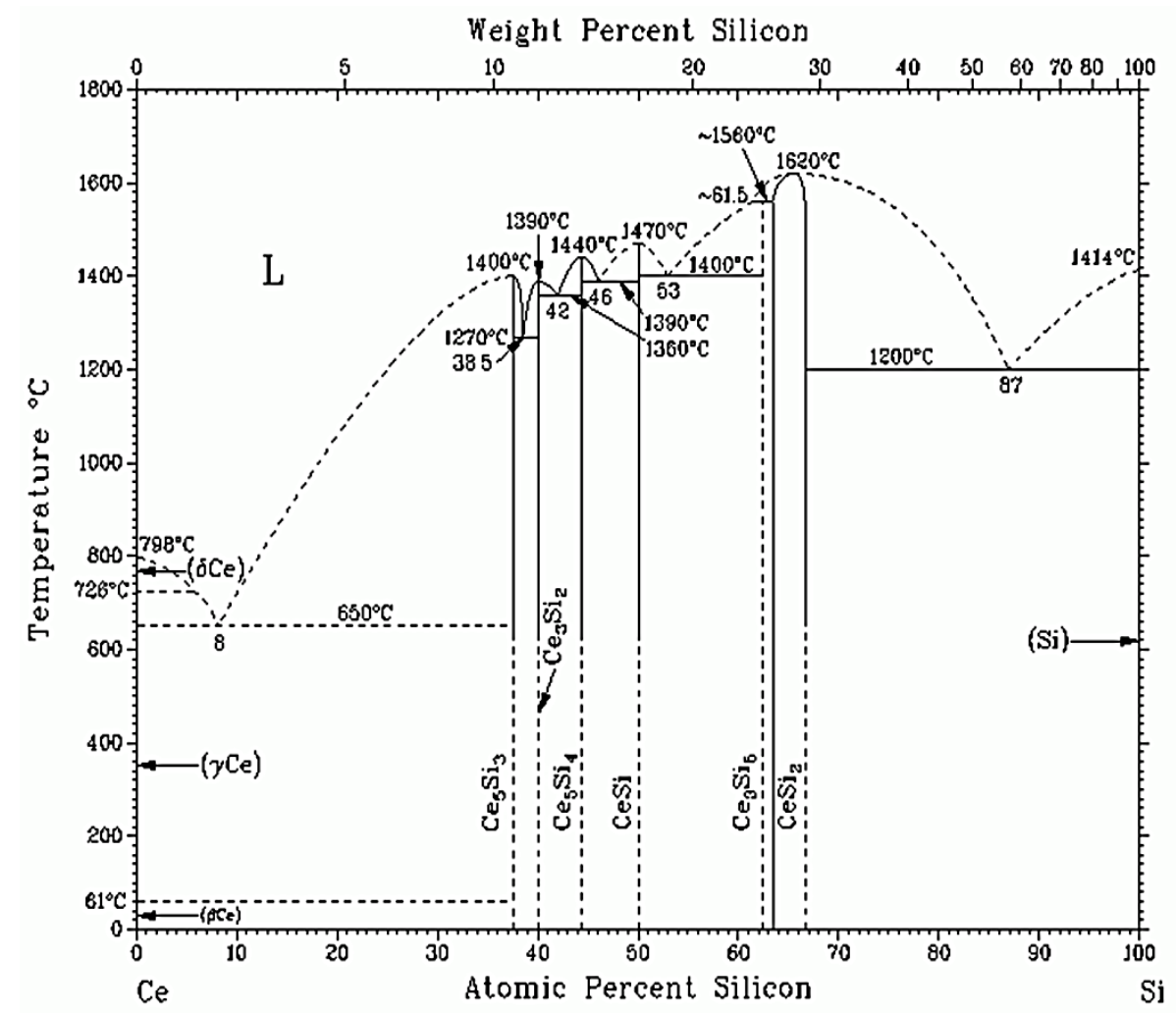

Figure 4-9. Cerium-silicon binary phase diagram [40].

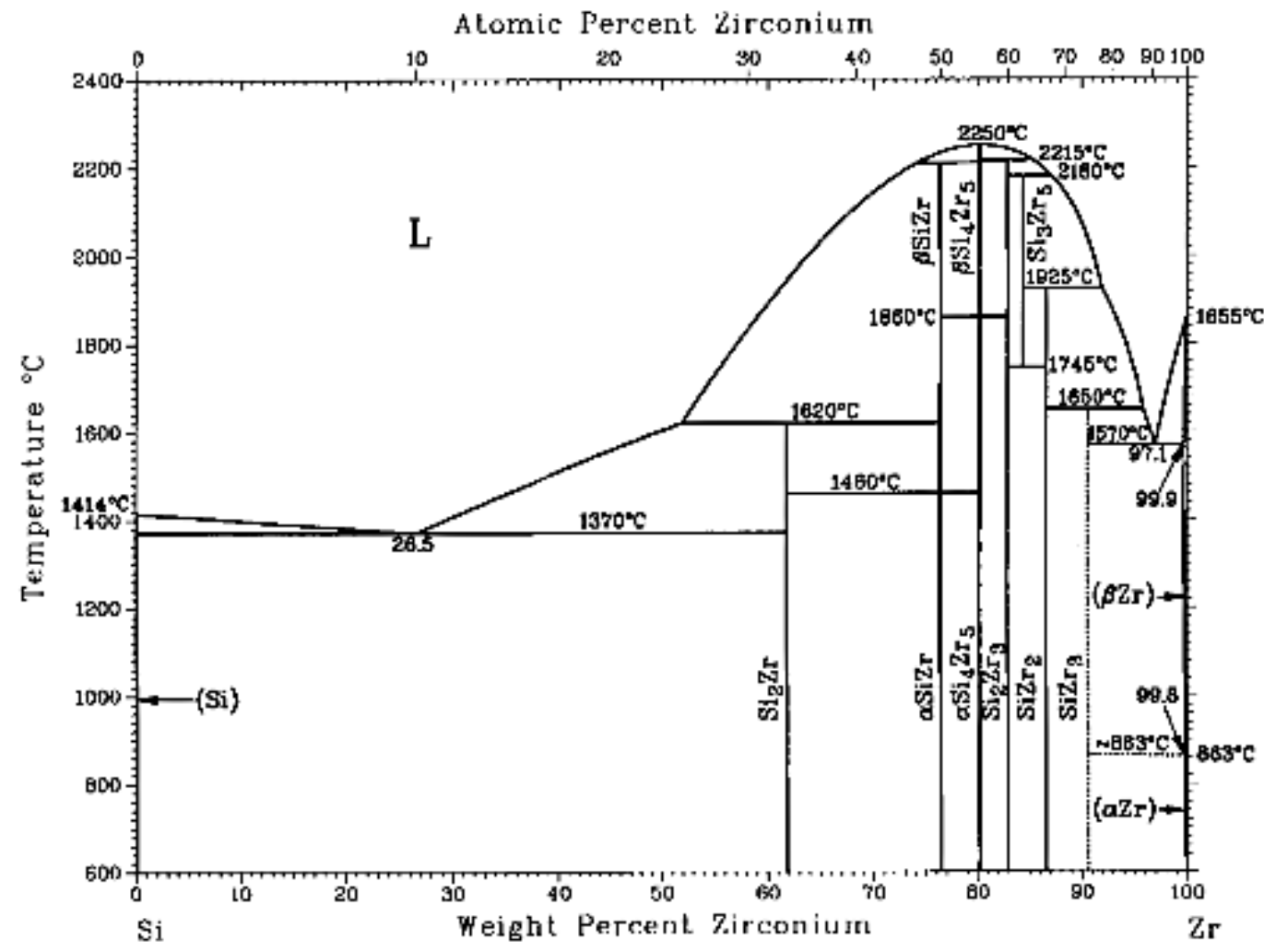

Figure 4-10. Zirconium-silicon binary phase diagram [41]. 


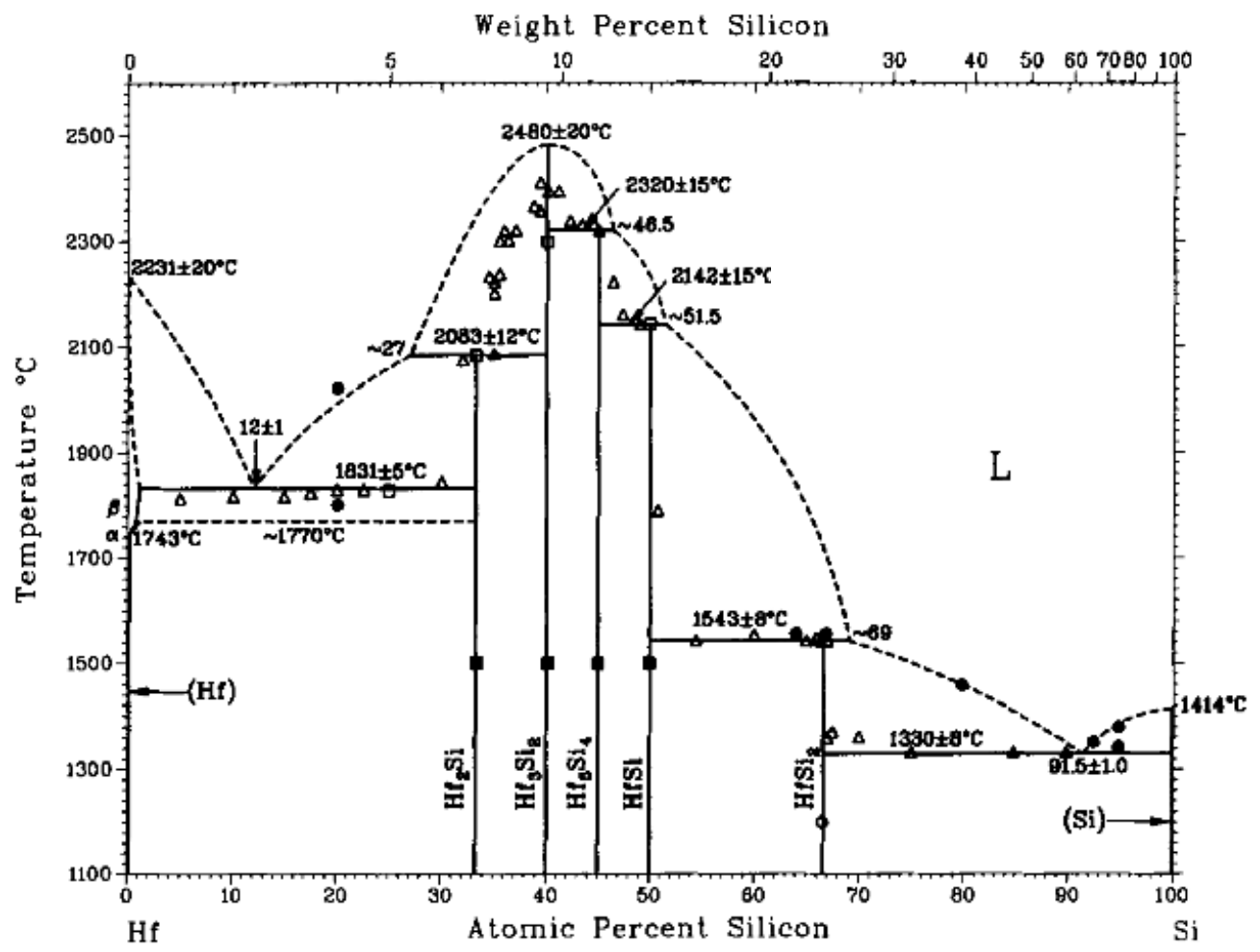

Figure 4-11. Hafnium-silicon binary phase diagram [42].

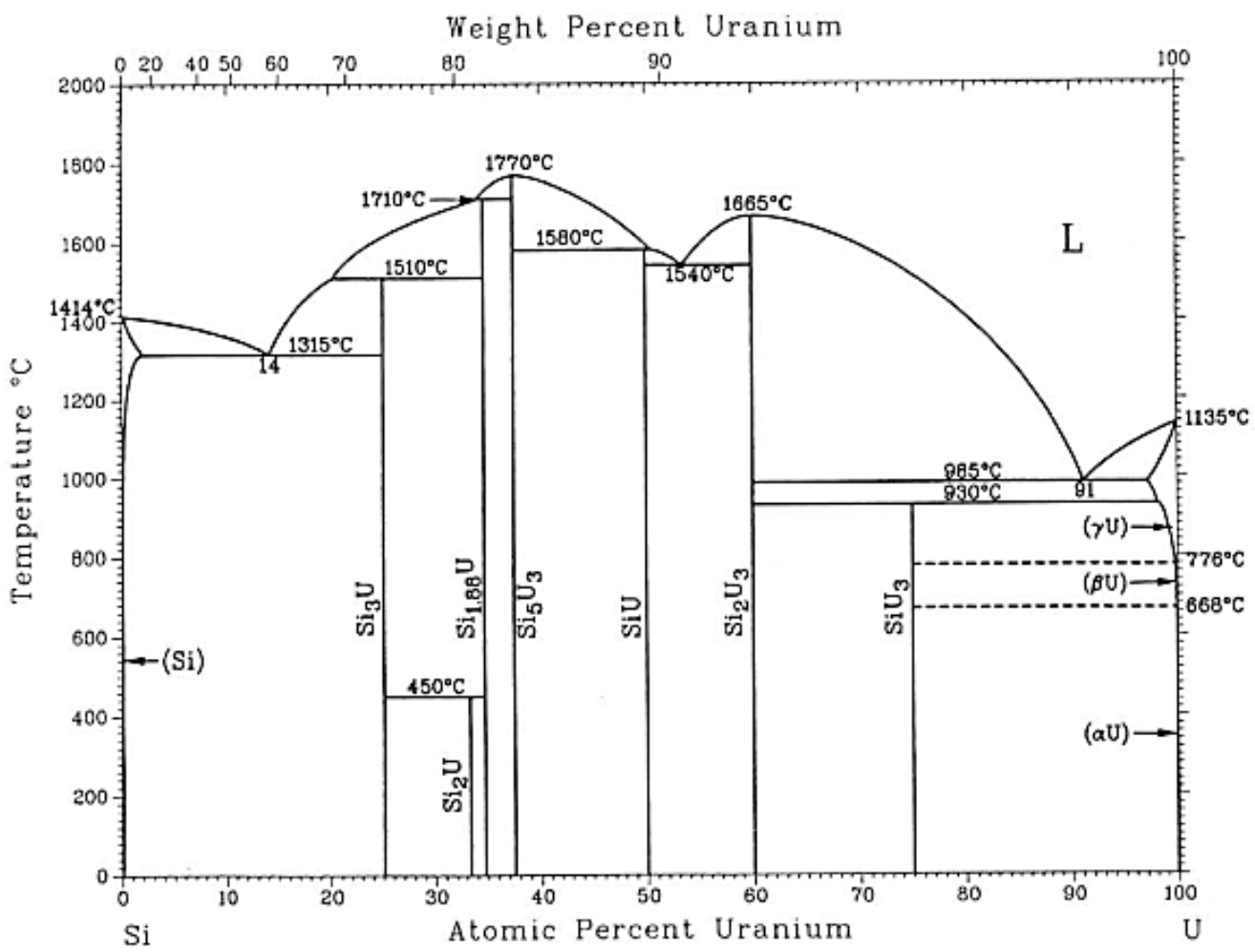

Figure 4-12. Uranium-silicon binary phase diagram [44]. 


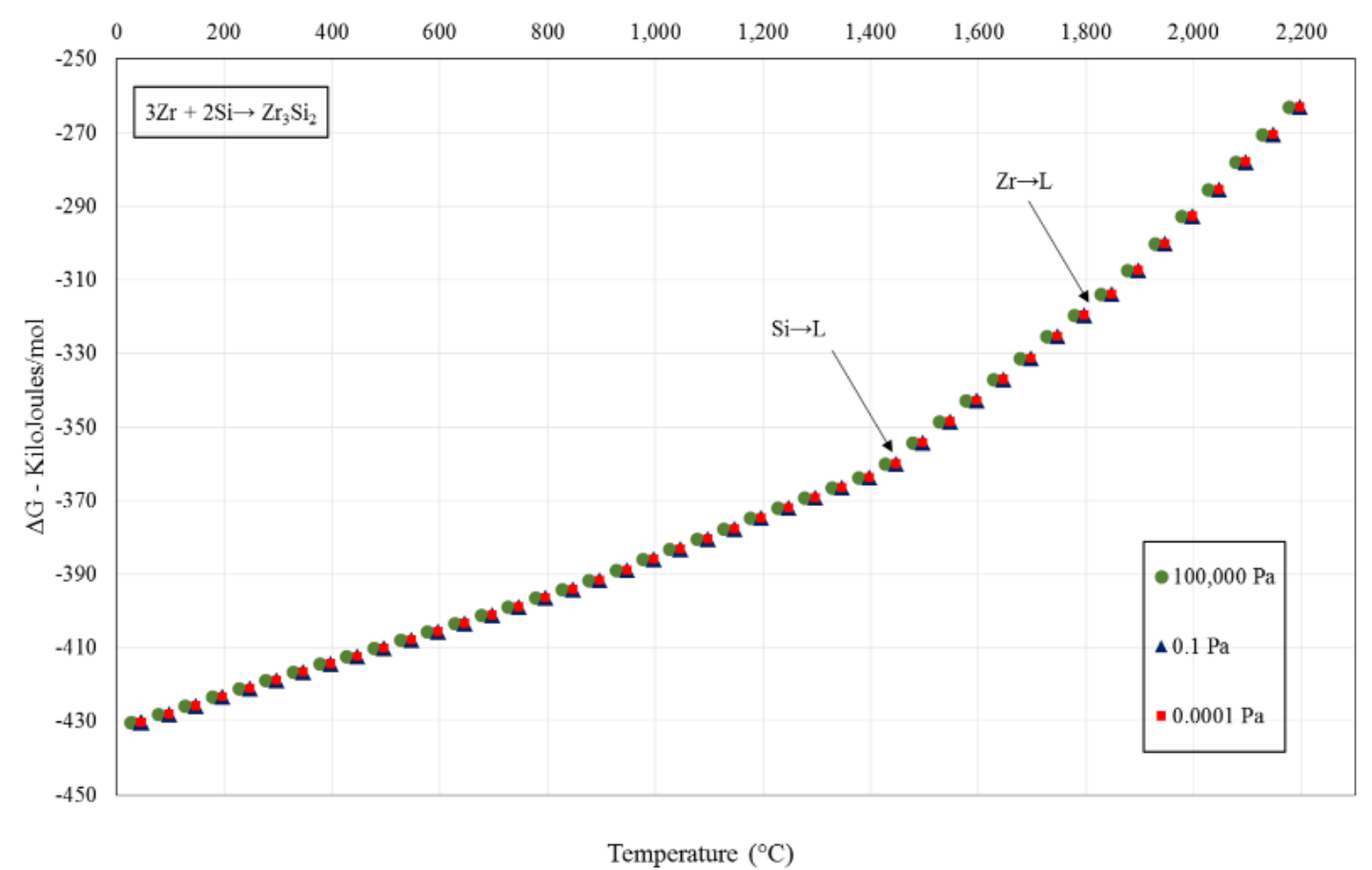

Figure 4-13. ThermoCalc simulation results on Gibbs free energy of $\mathrm{Zr}_{3} \mathrm{Si}_{2}$ with respect to temperature at $100,000 \mathrm{~Pa}, 0.1 \mathrm{~Pa}$, and $0.0001 \mathrm{~Pa}$.

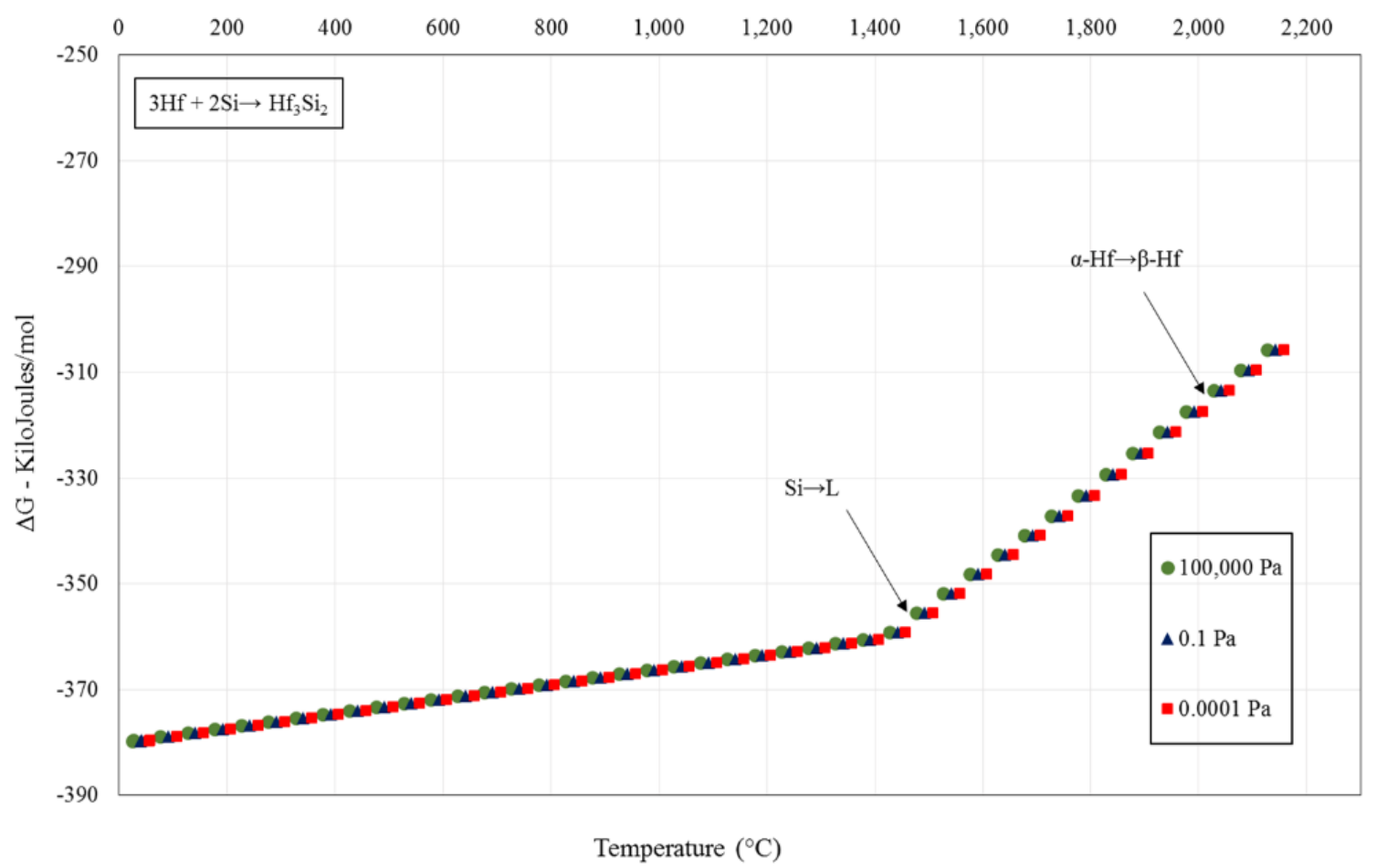

Figure 4-14. ThermoCalc simulation results on Gibbs free energy of $\mathrm{Hf}_{3} \mathrm{Si}_{2}$ with respect to temperature at 100,000 $\mathrm{Pa}, 0.1 \mathrm{~Pa}$, and 0.0001 $\mathrm{Pa}$. 


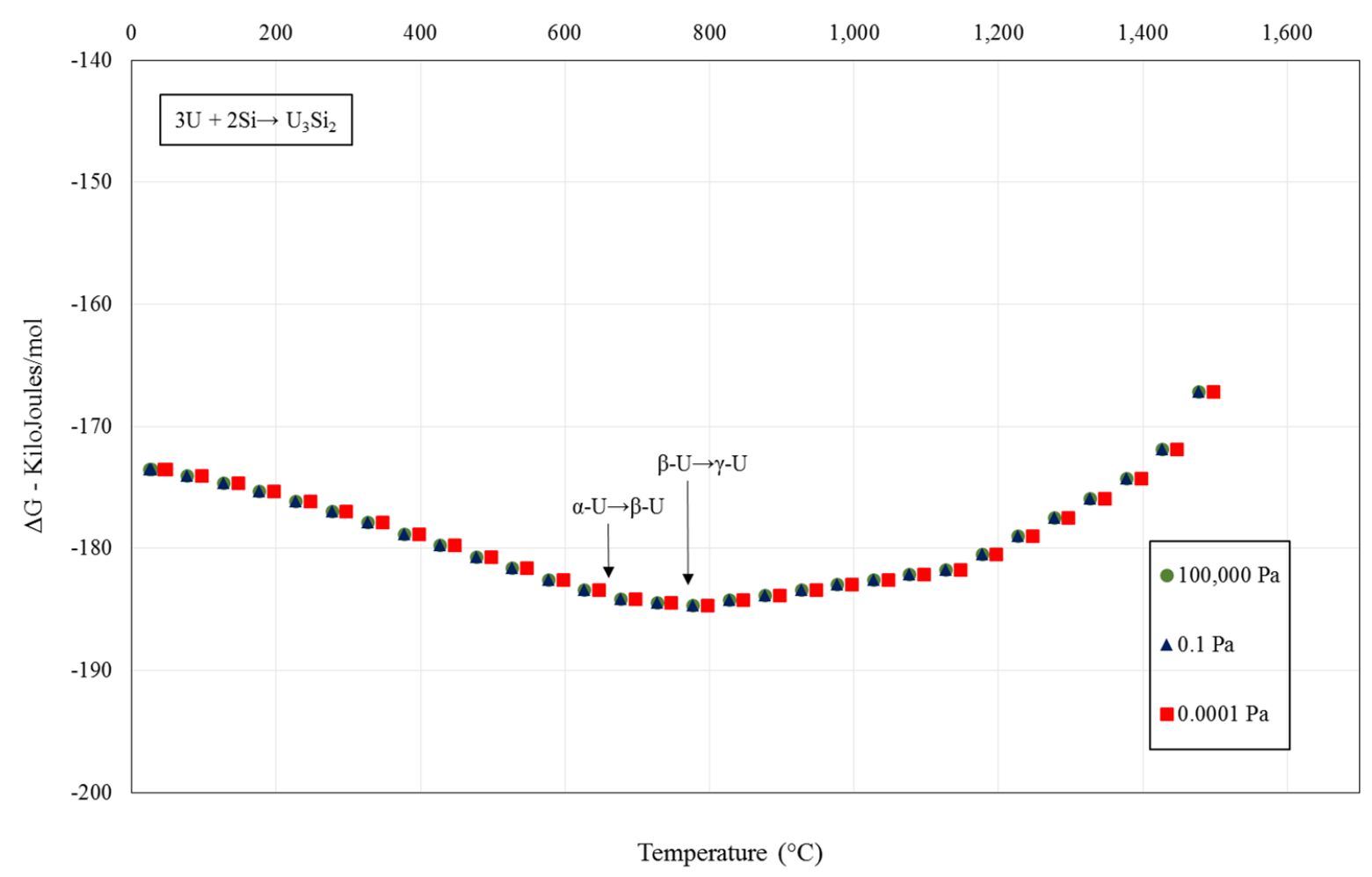

Figure 4-15. ThermoCalc simulation results on Gibbs free energy of $\mathrm{Hf}_{3} \mathrm{Si}_{2}$ with respect to temperature at 100,000 $\mathrm{Pa}, 0.1 \mathrm{~Pa}$, and 0.0001 $\mathrm{Pa}$. 


\section{CHAPTER 5}

EFFECTS OF LASER INTEARCTION ON U $\mathrm{Si}_{2}$ SURROGATES - CHARACTERIZATION

\section{Background}

The surrogate samples were synthesized using a laser beam source. Thus, understanding the interaction between the surrogate material and the laser source is crucial to develop suitable surrogates and implement the additive manufacturing process, as proposed in Objective 2. The surrogate samples prepared using the additive manufacturing process were characterized by means of electron microscopy, EDS, and XRD in order to verify the sample integrity and the different phases present. The laser-matter interaction is an interdisciplinary and complex process in which the surrogate powders melt and thermodynamically interact to form an end product. The characterization results provided novel data on $\mathrm{U}_{3} \mathrm{Si}_{2}$ surrogates undergoing a laser synthesis during this phase of the additive manufacturing process. The microstructural data acquired was crucial to incorporate modifications in order to explore and ultimately develop the additive fabrication process of nuclear fuel.

\section{Experiment Matrix}

After analyzing the thermodynamics of formation and crystallographic properties of different elements, cerium, zirconium, and hafnium were down selected to undergo a laser synthesis to produce uranium fuel surrogates. The elements were acquired in powder form and mixed in the proper stoichiometry to form the desired fuel surrogate compound. The powder mixtures were placed in tungsten crucibles to undergo the laser synthesis. To melt the surrogate fuel compounds, a laser energy source was employed. An industrial laser welder (LaserStar 990 Series) was used for the procedure, as an early desktop phase. The laser beam was operated under an argon atmosphere at different power outputs, which depend on the surrogate fuel to be melted. A preliminary phase, "phase zero," sought to test the experimental setup and to 
demonstrate the feasibility of the laser in melting hafnium, the surrogate with the highest melting point, which is $2,226.85{ }^{\circ} \mathrm{C}$ [67] . Phase I involved the experimental procedure to synthesize cerium-silicide, zirconium-silicide, and hafnium-silicide. Initially, when performing the laser melting, excessive sputtering of the sample powders occurred. To prevent loose powder from further sputtering outside of the testing crucible, the compound mass was reduced and also pressed to form a compact. The melting procedure consisted of striking a laser beam into the powder sample and shifting it to the surrounding areas while maintaining a molten pool of the compound. This approach achieved a fully molten compound on each experiment allowing the formation of different surrogate phases that were later characterized.

\section{Sample Preparation}

\section{Overview}

The sample preparation activities consist of different procedures such as handling, cutting, mounting, polishing, etching, and coating samples to properly prepare them for SEM analysis. These activities generally alter the specimens physically and chemically in order to render their microstructural features for further analysis. The main hazards associated with the sample preparation process may be the chemical hazards primarily arising from the use of acids employed for etching purposes. To perform sample preparation, adequate personal protective equipment was employed, and all equipment was operated in accordance with the manufacturer's instructions.

\section{Sectioning and Cutting}

The first step is to select a sample area to properly characterize the microstructure along with other features of interest in the selected material. It is recommended that the grain size measurements be performed on transverse sections and the general microstructure analyses on longitudinal sections in order to evaluate a larger portion of the sample. Sectioning and reducing the sample should be performed carefully to prevent alterations or potential damage to the 
structure of the material. An abrasive saw is generally recommended for metals and metal matrix composites, but the type of saw will ultimately depend on the material to be sectioned. A diamond saw is commonly used for ceramics, electronics, biomaterials, and minerals. Optimum cutting involves the proper selection of abrasive type, bonding, and size, as well as a proper cutting speed, load, and coolant. Table 5-1 displays the common abrasive blades used for metallographic sectioning. Table 5-2 shows diamond parameters for diamond saw cutting. Independent of the blade, cutting speed, or technique employed, a small amount of deformation will occur in the sample's surface. Nonetheless, it is highly encouraged to mitigate such deformations to acquire optimum microstructural data [68].

\section{Mounting}

The purpose of mounting a sample is to allow protection of the specimen's edge and to keep the integrity of the specific surface features of the material. It also fills voids in porous materials and improves handling for the next set of preparation procedures. The majority of metallographic mounting is performed by encapsulating the sample into a compression mounting compound (specifically, thermosets, including phenolics, epoxies, diallyl phthalates or thermoplastics-acrylics), casting into ambient castable mounting resins (including acrylic resins, epoxy resins, and polyester resins), and gluing with a thermoplastic glues. For compression mounting, phenolics are the most common, due to their low cost. Diallyl phthalates and epoxy resins are suitable when edge retention and harder mounts are required. Acrylic compression mounts are employed due to their clarity. Table 5-3 and Table 5-4 list the specific applications and the physical properties of the conventional compression mounting resins, respectively. Castable mounting resins are commonly used for electronic and ceramic materials. They can also be suitable to mount brittle and porous materials. Such compounds are generally composed of two solutions, one resin and one hardener. The average curing timeframes range from minutes to 
hours, faster curing resins produce a higher exothermic temperature that causes the mounting material to shrink away from the edge during curing [68]. Table 5-5 displays the basic properties of castable mounting resins.

\section{Grinding and Polishing}

Grinding, followed by polishing is generally performed to reduce the damage created by cutting and sectioning the sample. Planar grinding is completed by reducing the abrasive grit particle size sequentially in order to obtain a surface finish suitable for polishing. This step should be performed carefully to avoid generating additional damage other than produced during cutting. Grinding and polishing pressure is strictly linked to the axial force applied to the sample, along with the area of the specimen. For larger, hard samples, higher grinding pressures can increase stock removal rates but also tends to increase the surface and subsurface damage [68].

The disk rotation allows a variable velocity distribution. For high stock removal, a slower head speed relative to a higher base speed generates the most aggressive grinding operation. The main drawback is that the abrasive paper may not wear out uniformly, resulting in non-uniform removal across the sample's surface. Another drawback is that a higher velocity may produce substantial damage, especially in brittle areas. Placing the same velocity on head and base allows the retention of inclusions and brittle phases as well as for attaining a very uniform finish across the sample. The most common abrasive used in metal and polymer grinding is $\mathrm{SiC}$. $\mathrm{SiC}$ is an effective abrasive due to its hardness, and maintain a sharp cutting edge as it wears down during cutting. SiC abrasives are generally listed by their grit size. Table 5-6 displays the most common grit size with their respective particle size. For metallic specimens sequential grinding with $\mathrm{SiC}$ abrasive paper is the most efficient grinding process. Finer papers are also available for continued abrasive paper grinding (800 and 1,200 grit). In addition to the correct sequence and abrasive size selection, grinding parameters such as direction, load, and speed can affect the 
specimen flatness and depth of damage [68]. For ceramic composites, a rough grinding can be performed with a $15-30$ micron diamond on a metal mesh cloth in order to achieve optimum stock removal and to minimize surface and subsurface damage. Final polishing will remove only surface damage. It should not be employed to remove any damage originated from cutting or grinding. For ceramics, it is recommended to use low napped polishing pads using $1 \mu \mathrm{m}$ polycrystalline diamond alternating with colloidal silica [68].

\section{Etching}

The purpose of etching is to optically enhance microstructural features, such as grain size and phase features. Selective etching alters the aforementioned features based on composition, stress, or crystal structure. The conventional method for selective etching is chemical etching. However, different techniques such as molten salt, electrolytic, thermal and plasma etching have also found specialized applications. Table 5-7 displays some of the most common chemical etchants along with the specific application of each. Chemical etching selectively affects targeted microstructural features. It usually consists of a mixture of acids or bases, including oxidizing and reducing agents [68].

\section{Preliminary Laser Synthesis of Surrogate Materials}

\section{Experimental}

To verify the laser intensity, analyze the laser material-interaction, and test the experimental setup, a 316-stainless steel powder sample has been exposed to a laser energy source, showing that the laser powder and holding times were sufficient to melt the material. A 316-stainless steel powder sample ( $4 \mathrm{~g})$ was placed in an alumina crucible. An industrial laser welder (LaserStar 990 Series) was used for the additive manufactured surrogates, as an early desktop phase. The laser beam was operated with at power outputs ranging from 50-100 $\mathrm{W}$. The laser system includes an adjustable head, offering the flexibility of varying the source-to-sample 
distance during the welding procedure (Z-axis variation). The laser beam had a pulse width of approximately 2 milliseconds with an approximate beam diameter of $2 \mathrm{~mm}$ upon contact with the sample powder. The laser wavelength employed on this experimental procedure was of $1064 \mathrm{~nm}$. Molten material sputtering was observed immediately upon laser impact, and a solid compound was obtained upon solidification. After proving the laser's suitability for melting 316 stainless steel, a uranium-surrogate alloy synthesis phase followed. For this phase, hafnium $(2 \mathrm{~g})$ and silicon (2 g) in powder form were employed to attempt to form a hafnium silicide (HfSi 2 ) compound. During the process, sputtering was again observed, and the fabricated alloy solidified in different segments generated during sputtering. (See Figure 5-1) The properties of the target elements included in the experiments are listed in Table 5-8.

\section{Characterization}

The manufactured samples were sectioned and then mounted in an electrical conductive resin. The samples were polished and chemically etched (using a hydrofluoric acid medium) in order to enhance the microstructural features for scanning electron microscope (SEM) examination. The samples' morphologies, microstructures, and chemical analyses were examined using a JEOL scanning electron microscope (model JSM-6610-LV) coupled with energy dispersive x-ray spectroscopy (EDS). X-ray diffraction spectroscopy (XRD) was performed using a Rigaku Smartlab x-ray diffractometer. Figure 5-1 (a) displays the optical image of the as-synthesized hafnium silicide. Partially melted features and various cavities can be observed. Various partially melted pieces were observed in the crucible, this was expected because of sputtering that occurred due to the intermittent laser power applied. The synthesized Hf-Si piece in Figure 5-1 (a) was mounted after transversal sectioning and further prepared for SEM examination. Figure 5-1 (b) reveals the microstructure of a partially melted area on the left and a fully melted area on the right of the specimen. Figure 5-2 (a) displays the microstructure of 
a Hf-Si sample showing porosity and sintered or melted structures. During the laser synthesis, a portion of silicon did not react to form Hf-Si compounds. In the center of the sample, a large void can be observed, which was common on all shattered sample pieces fabricated in this early work. The large voids may possibly occur due to phase explosion during the laser-material interaction. The central voids, along with the plasma formation, are in agreement with Han et al. [69]. Phase explosion is a thermal effect observed during laser irradiation that accompanies the formation of a superheated liquid due to laser energy deposition. The generation and growth of nucleation in the super-heated material occurs next in the process, which concludes with the explosion of nucleation, generating a void or crater. The scanning electron microscopy images revealed partial sintering on both hafnium silicide and stainless steel samples. Figure 5-2 (b) displays the morphology of a 316 stainless steel sample, upon which some areas were not fully sintered; this sample shows signs of sputtering. Due to the high-power density of the laser beam, the target materials experienced an uneven heat distribution, causing sputtering and, sometimes, immediate vaporization of the material.

At high laser irradiation $\left(>10^{9} \mathrm{~W} / \mathrm{cm}^{2}\right)$ the vapor or ambient gas generated can become ionized. At this point, it is properly described as "plasma." As the temperature increases, collisions between thermal electrons and vaporized species produce a degree of ionization specified by the Saha equation [70]. The ionized gas strongly absorbs the laser radiation and expands within the laser beam path, shielding the target material from the incident laser beam [71].

The XRD analysis revealed the dissolution of hafnium and silicon, leading to the formation of $\mathrm{HfSi}_{2}$ as displayed by the peaks in Figure 5-3 (Card: ICDD - PDF-2 00-038-1373). Not all the available silicon powder reacted with hafnium; the remainder was left in its pure 
elemental form as confirmed by the peaks in Figure 5-3 and the EDS analysis (Card: ICDD PDF-2 00-005-0565). The carbon peaks identified by x-ray diffraction and semi-quantitative EDS analysis (Card: ICDD - PDF-2 00-041-1487) are an artifact, as they can be attributed to the conductive resin holder seen in Figure 5-2 (a). Table 5-9 displays the chemical composition of a hafnium silicide sample. Figure 5-4 displays the microstructure of the sample upon which the chemical analysis shown in Table 5-9 was performed. The presence of $\mathrm{Sn}$ is attributed to the conductive resin holder, some of which was incorporated onto the sample.

\section{Summary}

The melting of stainless steel and hafnium silicide by means of a laser energy beam proved to be feasible, and the experimental setup can be used for further desktop experimentation. Laser synthesis was validated as a suitable methodology to alloy $\mathrm{Hf}$ and Si to form hafnium silicide ( $\left.\mathrm{HfSi}_{2}\right)$. Nonetheless, additional development needs to be implemented in order to fully sinter the material into a homogenous mixture and to validate this method for different stoichiometries. Further work may include a decrease in the laser power output and an increase in the welding time to prevent sputtering and void formation. Additional XRD analyses on the remaining sputtered specimens will be performed and may enhance our understanding of the composition of the fabricated samples.

\section{Characterization of Downselected Surrogates}

\section{Ce-Si}

A non-radioactive surrogate material with comparable properties to those of uranium is cerium. $\mathrm{Ce}_{3} \mathrm{Si}_{2}$ is isostructural with $\mathrm{U}_{3} \mathrm{Si}_{2}$ and has been previously used as a $\mathrm{U}_{3} \mathrm{Si}_{2}$ surrogate in studies of processing techniques, oxidation, corrosion, and transport properties [6]. The laser synthesis of cerium powders was performed on a benchtop setup on the early stages of development of an additive manufacturing process. The melting points of cerium and silicon are 
$798^{\circ} \mathrm{C}[40]$ and $1,414^{\circ} \mathrm{C}[40]$ respectively, thus, the laser power output employed yielded enough heat to overcome $798^{\circ} \mathrm{C}$ and produce a molten pool of the compound. After synthesizing the sample, it was cooled down at room temperature under an argon atmosphere.

The samples were extracted and prepared for SEM examination. Figure 5-5 (a) displays the microstructure of a partly sintered sample. Due to the experimental conditions (most probably a too-high laser intensity), some areas were exposed to different heating gradients, providing a non-homogenous compound mixture. Figure 5-5 (b) displays voids on the sample's morphology. The void formation could be attributed to the uneven heat distribution during the laser synthesis. Figure 5-5 (c) portrays a back-scatter electron micrograph of the sample upon which EDS analyses were performed. Since EDS is a semi-quantitative method, all phases evaluated with EDS were analyzed and then confirmed with XRD. The mass contrast allowed to identification of different phases of the Ce-Si system. Based on the quantification analyses from EDS, Spots 1 and 2 suggested the presence of a CeSi phase. Spots 3 and 4 revealed the presence of high concentrations of silicon in its elemental state, which suggests that silicon remained unreacted in those locations. Figure 5-5 (d) displays a back-scatter electron micrograph depicting the morphology of the melted sample. The EDS quantification analyses revealed the presence of a $\mathrm{CeSi}_{2}$ phase on Spots 5 and 6. Additional spots suggested the presence of $\mathrm{CeSi}$ and elemental silicon on Spots 8 and 9, respectively. The quantification results from the chemical analyses performed are displayed in Table 5-10.

XRD analyses were executed on the cerium-silicon synthesized sample. Figure 5-6 displays the exact location where the diffraction pattern was collected. The XRD pattern is displayed in Figure 5-7. Additional XRD analyses were performed on powder that was generated from sputtering along the laser synthesis. Figure 5-8 showcases the XRD pattern collected from 
the synthesized powder. The XRD analyses confirmed the presence of the CeSi (DB card : 00018-0320), $\mathrm{CeSi}_{2}$ (DB card : 00-006-0485), $\mathrm{Ce}_{5} \mathrm{Si}_{3}$ (DB card : 00-062-0631) pertaining to the Ce-Si system. Additionally, silicon (DB card : 00-005-0565) was present in its elemental state, indicating that it remained unreacted. No findings of $\mathrm{Ce}_{3} \mathrm{Si}_{2}$ were reported under this experimental procedure. However, the phases $\mathrm{CeSi}, \mathrm{CeSi}_{2}, \mathrm{Ce}_{5} \mathrm{Si}_{3}$ and silicon found are in close agreement with other work seeking to produce cerium silicide [6]. The formation of different phases of the Ce-Si system proved the feasibility of the laser synthesis as an effective power source. The results presented on Ce-Si will enhance our understanding and will be employed to make assumptions and comparisons while working with uranium-bearing compounds in future research.

\section{Zr-Si}

Zirconium was down selected as a surrogate material due to its chemical and crystallographic properties, which are comparable to those of uranium. Research studies have proven the feasabiltiy of employing $\mathrm{ZrO}_{2}$ as surrogate material for uranium fuel in TRISOcoated particles [8][72]. Zirconium and silicon powders were mixed in the proper stoichiometric proportions to undergo a laser synthesis process and ultimately produce $\mathrm{Zr}_{3} \mathrm{Si}_{2}$. In the early stages of developing an additive manufacturing process, this experimentation was performed on a small scale solely to provide the proof of concept in forming the desired surrogate compound $\mathrm{Zr}_{3} \mathrm{Si}_{2}$. For the experimental procedure, a tungsten crucible was filled with the sample powders. As the laser interacted with the sample, excessive sputtering was observed. Hence, the mass of the sample powders was reduced to minimize sputtering and allow the formation of a melted pool of the compound. Upon contact with the sample powder the beam was spread in order to dissipate heat over a wider area. Like in the previous experiment with cerium, the objective was to form a molten pool of the compound, then allow it to gradually cool down and solidify under 
an argon atmosphere. The samples were sectioned and prepared for SEM. Figure 5-9 displays the SEM micrographs of the laser synthesis of Zr-Si. Figure 5-9 (a) portrays the microstructure of a partially melted sample upon which different spots were evaluated by EDS. Table 5-9 displays the quantification results from the EDS analyses on Zr-Si. In Spots 1, 2, 3, and 4, the chemical analyses performed suggest the presence of a $\mathrm{Zr}_{3} \mathrm{Si}_{2}$ phase. All chemical analyses in this section were analyzed and then confirmed by XRD. It is assumed that a high laser intensity generated an uneven heat distribution throughout the synthesis process, allowing the formation of a nonhomogenous morphology in the fabricated samples. Figure 5-9 (b) displays the presence of large voids in the morphology of sample ZS-S1. EDS analysis was performed on Spots 5, 6, 7, and 8, and the results indicate the presence of a $\mathrm{Zr}_{3} \mathrm{Si}_{2}$ phase. Back-scatter electron microscopy was employed to evaluate the different phases of the $\mathrm{Zr}$-Si system that were present on the synthesized samples. Figure 5-9 (c) displays the grain structure of the $\mathrm{Zr}_{3} \mathrm{Si}_{2}$ phase. Individual grains on Spots 9, 10, and 11 were evaluated by EDS, which confirmed the presence of $\mathrm{Zr}_{3} \mathrm{Si}_{2}$ in the compound. The grain boundaries displayed a lighter tonality in the micrographs; such mass contrast indicated a different composition from the grains evaluated. The EDS analysis confirmed the presence of high concentrations of zirconium on the grain boundaries, suggesting that it either remained unreacted or formed a zirconium-rich silicide compound. The formation of silicon and zirconium rich phases can be attributed to the inhomogeneous heat distribution from the laser source, which can instantly vaporize any element, generating hyper and hypostoichiometric phases. Figure 5-9 (d) displays a back-scatter electron micrograph showing both grains and grain boundaries present on silicide Sample SZ-S2. The EDS analysis suggests that the composition of the grain boundaries displayed on Spots 12, 13, and 14 contain a high 
concentration of zirconium. Spots 15 and 16 confirmed the presence of $\mathrm{Zr}_{3} \mathrm{Si}_{2}$ on the evaluated grains.

XRD analyses were performed on the Zr-Si surrogate samples. Figure 5-10 (a) displays the exact location where the XRD pattern was collected in Sample ZS-S1. Figure 5-10 (b) shows a partially sintered location where the XRD pattern was collected in Sample ZS-S2. The XRD patterns on samples ZS-S1 and ZS-S2 are displayed in Figures 5-11 and 5-12, respectively. The XRD results confirmed the presence of $\mathrm{ZrSi}$ (DB card: 01-072-2031), $\mathrm{ZrSi}_{2}$ (DB card: 01-0708990), $\mathrm{Zr}_{2} \mathrm{Si}$ (DB card: 00-025-0757), and $\mathrm{Zr}_{3} \mathrm{Si}_{2}$ (DB card: 00-014-0368) from to the $\mathrm{Zr}-\mathrm{Si}$ system. Moreover, silicon (DB card: 00-027-1402) was present in its elemental state, indicating that it remained unreacted during the laser synthesis process. The XRD pattern for $\mathrm{Zr}_{3} \mathrm{Si}_{2}$ is consistent with other research works fabricating the compound via a hot isostatic pressing methodology [44]. With the exception of $\mathrm{Zr}_{5} \mathrm{Si}_{3}$, no other phases were reported. $\mathrm{The} \mathrm{Zr}_{3} \mathrm{Si}_{2}$ fabricated under a laser synthesis was not a pure phase. Some portions are composed by $\mathrm{Zr}_{3} \mathrm{Si}_{2}$ phase but different secondary phases from the $\mathrm{Zr}$-Si system were identified by means of XRD and chemical analyses.

Nonetheless, previous research works that sought to develop $\mathrm{U}_{3} \mathrm{Si}_{2}$ proved that is essentially impossible to fabricate an alloy at the exact stoichiometry of the compound and of such atomistic homogeneity that only a pure phase is present [1]. Thus, this novel laser synthesis of surrogate nuclear fuel proved the feasibility of forming a zirconium silicide $\left(\mathrm{Zr}_{3} \mathrm{Si}_{2}\right)$ compound and with further optimization of the laser parameters, homogeneity of the $\mathrm{Zr}_{3} \mathrm{Si}_{2}$ phase can be found. The acquired results from this surrogate experimental procedure set the foundation for the development of experiments to form $\mathrm{U}_{3} \mathrm{Si}_{2}$ fuel by means of a laser synthesis. 


\section{Hf-Si}

Hafnium was down-selected as a potential surrogate material for this research work due to its crystallographic and thermal properties, which are comparable to those of uranium [45]. Previous research work have successfully employed hafnium as a uranium surrogate to simulate uranium fuel debris for clean-up projects and high-level waste disposal [45][46]. Hafnium and silicon powders were placed in a tungsten crucible to undergo laser synthesis. From the downselected surrogates, hafnium had the highest melting point, which is $2,226.85^{\circ} \mathrm{C}$ [67]. Thus, like in the previous experiments with surrogates, the laser power output was configured to surpass the highest melting point of both elements and allow the formation of a molten pool of the compound. Following the laser synthesis, the molten compound was allowed to cool at room temperature always conserving an argon atmosphere.

The fabricated samples were prepared for SEM examination and later characterized. Figure 5-13 (a) displays the morphology of the hafnium silicide sample HS-S1. It can be observed that, in some areas, the compound was partially melted. It is assumed that the heat dissipation was not uniform throughout the sample, allowing the formation of voids and other defects leading to a brittle structure. Figure 5-13 (b) displays partial melting of the compound. The middle area shows several cavities where the heat dissipation was uneven and did not allow the formation of a fully molten compound. Figure 5-13 (c) displays the locations where EDS analysis was performed on individual sections of the compound, which confirmed the presence of the $\mathrm{Hf}_{3} \mathrm{Si}_{2}$ phase. The chemical analysis quantification results suggest the presence of $\mathrm{Hf}_{3} \mathrm{Si}_{2}$ on Spots 1, 2, and 3. Table 5-12 displays the quantification results from the EDS analyses performed on hafnium silicide. Figure 5-13 (d) displays the chemical analyses performed on additional sections of Sample HS-S1. Spots 4 and 5 display a higher concentration of hafnium, 
suggesting the presence of the $\mathrm{Hf}_{3} \mathrm{Si}_{2}$ phase. The quantification analysis on Spots 6, 7, and 8 suggest the presence of a HfSi phase, which later was confirmed by XRD.

The Hf-Si surrogate HS-S1 was evaluated with XRD. Figure 5-14 displays an SEM micrograph with exact locations from which the XRD patterns were collected. The collected XRD patterns on Sample HS-S1 are being displayed on Figures 5-15 and 5-16. The XRD analyses confirmed the presence of the surrogate phase $\mathrm{Hf}_{3} \mathrm{Si}_{2}$ (DB card: 01-070-2839) along with secondary phases from the hafnium-silicon system, including $\mathrm{Hf}_{5} \mathrm{Si}_{3}$ (DB card: 03-0653618) and HfSi (DB card: 01-070-2838), in addition to carbon (DB card: 00-023-0064) which was attributed to the sample holder. The $\mathrm{Hf}_{3} \mathrm{Si}_{2}$ surrogate compound fabricated under the laser synthesis was not a pure phase. However, it proved the feasibility of acquiring the desired stoichiometry, $\mathrm{Hf}_{3} \mathrm{Si}_{2}$, to continue incorporating modifications to the AMAFT process. Based on the XRD results along with chemical analyses, only phases in equilibrium were formed along the manufacturing process for all surrogates. The absence of non-equilibrium phases is attributed to the fast cooling occurring during the AMAFT process, allowing only the most stable phases to fully form. Further study on the phases formed during the process may be explored employing slow cooling rates under thermogravimetric analysis (TGA).

\section{Conclusion}

A preliminary set of experiments was carried to test the experimental setup and demonstrated the feasibility of the laser intensity in melting metals and forming a hafnium silicide in the form of HfSi. The laser synthesis on cerium allowed the formation of different phases from the $\mathrm{Ce}-\mathrm{Si}$ system including $\mathrm{CeSi}, \mathrm{CeSi}_{2}$, and $\mathrm{Ce}_{5} \mathrm{Si}_{3}$. This was confirmed through EDS and XRD analyses. Although $\mathrm{Ce}_{3} \mathrm{Si}_{2}$ was not identified, the formation of cerium silicide phases proved the feasibility of laser synthesis as an effective power source to alloy and form cerium silicide compounds. This novel laser methodology proved to be a successful approach 
to achieve the desired stoichiometry of synthesized $\mathrm{Zr}_{3} \mathrm{Si}_{2}$. The quantification results from EDS suggested the presence of the $\mathrm{ZrSi}, \mathrm{ZrSi}_{2}, \mathrm{Zr}_{2} \mathrm{Si}$, and $\mathrm{Zr}_{3} \mathrm{Si}_{2}$ phases from the $\mathrm{Zr}-\mathrm{Si}$ system; this was also confirmed by XRD. In addition, hafnium silicide was effectively fabricated by means of laser synthesis, producing a fuel surrogate compound, $\mathrm{Hf}_{3} \mathrm{Si}_{2}$. The quantification results from EDS suggested the presence of the $\mathrm{Hf}_{5} \mathrm{Si}_{3}, \mathrm{HfSi}$, and $\mathrm{Hf}_{3} \mathrm{Si}_{2}$ phases from the Hf-Si system. This was also confirmed by the XRD analyses.

The surrogate samples were not in a pure phase, based on the XRD and EDS analyses, secondary phases were present from each independent system (Ce-Si, $\mathrm{Zr}-\mathrm{Si}$, and Hf-Si). Although previous studies showed difficulties in fabricating alloys with an exact stoichiometry and only a pure phase present [1], further modifications in the laser parameters and experimental setup will be incorporated in order to minimize the presence of secondary phases.

As a deliverable for Objective 2, all of the surrogate samples were synthesized using a laser beam and then characterized by means of SEM, EDS, and XRD. The characterization results on the microstructure allowed to determine the suitability of the samples after the laser synthesis. They confirmed the sample's integrity along with the formation of the desired compounds $\left(\mathrm{Zr}_{3} \mathrm{Si}_{2}\right.$ and $\left.\mathrm{Hf}_{3} \mathrm{Si}_{2}\right)$. In addition, the characterization results were crucial to improve the material synthesis and to incorporate modifications to ultimately develop the AMAFT process.

Among the selected surrogates, $\mathrm{Zr}_{3} \mathrm{Si}_{2}$ was the most relevant to analyze due to its welldefined morphological features. As displayed in Figures 5-9 (c) and 5-9 (d) several $\mathrm{Zr}_{3} \mathrm{Si}_{2}$ grains compose the matrix of the compound. The laser heating parameters were suitable to induce nucleation along with grain growth, allowing the formation of the desired phase. Besides validating the concept of laser synthesis on surrogate silicide fuel, the formation of $\mathrm{Zr}_{3} \mathrm{Si}_{2}$ can 
also be incorporated in different industry applications [44]. Additional development will be implemented to fully sinter the compound in a homogenous mixture to validate this methodology. Further experimental equipment modifications will seek to optimize the laser power output and exposure times to ultimately prevent sputtering and eliminate void formation. Nonetheless, the results from the surrogate compounds have provided highly useful microstructural data for designing the experimental set up to produce uranium compounds by means of an additive manufacturing process. 
Table 5-1. Abrasive blade selection guidelines [68].

\begin{tabular}{ccc}
\hline Alloy & Classification & Abrasive/Bond \\
\hline Aluminum, brass, zinc & Soft non-ferrous & $\mathrm{SiC} /$ rolled rubber \\
Heat treated alloys & Hard non-ferrous & Alumina/ rubber resin \\
$<$ Rc 45 Steel & Soft ferrous & Alumina/ rubber resin \\
$>$ Rc 45 Steel & Hard ferrous & Alumina/ rubber resin \\
Super alloys & High Ni-Cr alloys & $\mathrm{SiC} /$ rolled resin \\
\hline
\end{tabular}

Table 5-2. Diamond wafer blade selection guidelines [68].

\begin{tabular}{ccccc}
\hline Material & Characteristic & Speed (rpm) & Load (g) & Blade (grit) \\
\hline Silicon substrate & Soft/brittle & $<300$ & $<100$ & Fine \\
Gallium substrate & Soft/brittle & $<200$ & $<100$ & Fine \\
Boron composites & Very brittle & 500 & 250 & Fine \\
Ceramic composites & Very brittle & 1000 & 500 & Fine \\
Glasses & Brittle & 1000 & 500 & Fine \\
Minerals & Friable/brittle & $>1500$ & $>500$ & Fine \\
Alumina Ceramic & Hard/tough & $>1500$ & $>500$ & Medium \\
Zirconia & Hard/tough & $>3500$ & $>800$ & Medium \\
Silicon nitride & Hard/tough & $>3500$ & $>800$ & Medium \\
Metal matrix & - & $>3500$ & $>500$ & Medium \\
composites & - & & & \\
\hline
\end{tabular}


Table 5-3. Compression mounting application properties [68].

\begin{tabular}{ccccc}
\hline Criteria & Phenolics & Acrylics & Epoxy & $\begin{array}{c}\text { Diallyl } \\
\text { Phthalates }\end{array}$ \\
\hline Cost & Low & Moderate & Moderate & Moderate \\
Ease of use & Excellent & Moderate & Good & Good \\
Color availability & Yes & No & No & No \\
Cycle times & Excellent & Moderate & Good & Good \\
Edge retention & Fair & Good & Excellent & Excellent \\
Clarity & None & Excellent & None & None \\
Hardness & Low & Good & High & High \\
\hline
\end{tabular}

Table 5-4. Compression mounting resin physical properties [68].

\begin{tabular}{|c|c|c|c|c|}
\hline Resin & Phenolics & Acrylics & Epoxy & $\begin{array}{c}\text { Diallyl } \\
\text { Phthalates }\end{array}$ \\
\hline Form & Granular & Powder & Granular & Granular \\
\hline $\begin{array}{l}\text { Specific gravity } \\
\left(\mathrm{g} / \mathrm{cm}^{3}\right)\end{array}$ & 1.4 & 0.95 & $1.75-2.05$ & $1.7-1.9$ \\
\hline Colors & $\begin{array}{l}\text { Black, red, } \\
\text { green }\end{array}$ & Clear & Black & Blue \\
\hline $\begin{array}{c}\text { Shrinkage } \\
\text { (compression - } \\
\text { in/in) }\end{array}$ & 0.006 & - & $0.001-0.003$ & $\begin{array}{l}0.001- \\
0.003\end{array}$ \\
\hline $\begin{array}{l}\text { Coefficient of linear } \\
\text { thermal expansion }\end{array}$ & 50 & - & 28 & 19 \\
\hline Chemical resistance & $\begin{array}{l}\text { Glycol, } \\
\text { petrochemicals, } \\
\text { solvents, some } \\
\text { acids and bases }\end{array}$ & $\begin{array}{l}\text { Alcohol, dilute } \\
\text { acids \& alkalis. }\end{array}$ & $\begin{array}{l}\text { Solvents, } \\
\text { acids, alkalis }\end{array}$ & $\begin{array}{l}\text { Solvents, } \\
\text { acids, } \\
\text { alkalis }\end{array}$ \\
\hline $\begin{array}{l}\text { Molding } \\
\text { temperature }\end{array}$ & $150-165^{\circ} \mathrm{C}$ & - & $143-177^{\circ} \mathrm{C}$ & $\begin{array}{c}160-177 \\
{ }^{\circ} \mathrm{C}\end{array}$ \\
\hline Molding pressure & $21-28 \mathrm{MPa}$ & - & $17-28 \mathrm{MPa}$ & $\begin{array}{c}24-41 \\
\mathrm{MPa}\end{array}$ \\
\hline Hardness & - & Rockwell M63 & Barcol 72 & - \\
\hline $\begin{array}{c}\text { Curing time (1/2" } \\
\text { mount @ temp. and } \\
\text { pressure) }\end{array}$ & $90-120 \mathrm{sec}$ & $2-4 \min$ & $90-120 \mathrm{sec}$ & $90-120 \mathrm{sec}$ \\
\hline
\end{tabular}


Table 5-5. Castable mounting resin properties [68].

\begin{tabular}{cccc}
\hline Criteria & Epoxy & Acrylic & Polycast Resin \\
\hline Type & $\begin{array}{c}\text { Epoxy resin and } \\
\text { hardener }\end{array}$ & Acrylic resin and powder & $\begin{array}{c}\text { Polyester resin } \\
\text { and hardener }\end{array}$ \\
Peak temperature & $28{ }^{\circ} \mathrm{C}$ & $27^{\circ} \mathrm{C}$ & $38{ }^{\circ} \mathrm{C}$ \\
Cure time & $6-8$ hours & $5-8$ minutes & $6-8$ hours \\
\hline
\end{tabular}

Table 5-6. Particle size vs. common grit sizes for abrasive papers [68].

\begin{tabular}{ccc}
\hline European P-Grade & Standard Grit & Median Diameter (microns) \\
\hline P-60 & 60 & 250 \\
P-80 & 80 & 180 \\
P-100 & 100 & 150 \\
P-120 & 120 & 106 \\
P-150 & 150 & 90 \\
P-180 & 180 & 75 \\
P-220 & 220 & 63 \\
P-240 & 240 & 58.5 \\
P-280 & - & 52.2 \\
P-320 & 280 & 46.2 \\
P-360 & 320 & 40.5 \\
P-400 & - & 35 \\
P-500 & 360 & 30.2 \\
P-600 & 400 & 25.75 \\
P-800 & - & 21.8 \\
P-1,000 & 500 & 18.3 \\
P-1,200 & 600 & 15.3 \\
P-2,400 & 800 & 6.5 \\
P-4,000 & 1,200 & 2.5 \\
\hline
\end{tabular}


Table 5-7. Common chemical etchants [68].

\begin{tabular}{|c|c|c|c|}
\hline Etchant & Composition & Application & Conditions \\
\hline & $190 \mathrm{~mL}$ distilled water & & \\
\hline Keller's Etch & $\begin{array}{l}5 \mathrm{~mL} \text { nitric acid } \\
3 \mathrm{~mL} \text { hydrochloric acid } \\
2 \mathrm{~mL} \text { hydrofluoric acid }\end{array}$ & Aluminum alloys & $\begin{array}{l}\text { 10-30 second } \\
\text { immersion }\end{array}$ \\
\hline $\begin{array}{l}\text { Kroll's } \\
\text { Reagent }\end{array}$ & $\begin{array}{l}92 \mathrm{~mL} \text { distilled water } \\
6 \mathrm{~mL} \text { nitric acid } \\
2 \mathrm{~mL} \text { hydrofluoric acid }\end{array}$ & Titanium & 15 seconds \\
\hline Nital & $\begin{array}{l}100 \mathrm{~mL} \text { ethanol } \\
1-10 \mathrm{~mL} \text { nitric acid }\end{array}$ & $\begin{array}{l}\text { Carbon steels, tin, } \\
\text { nickel alloys }\end{array}$ & Seconds to minutes \\
\hline $\begin{array}{l}\text { Kalling's } \\
\text { Reagent }\end{array}$ & $\begin{array}{l}40 \mathrm{~mL} \text { distilled water } \\
2 \mathrm{~g} \text { of copper chloride } \\
\left(\mathrm{CuCl}_{2}\right) \\
40 \mathrm{~mL} \text { hydrochloric acid } \\
40-80 \mathrm{~mL} \text { ethanol }(85 \%)\end{array}$ & $\begin{array}{l}\text { Wrought stainless } \\
\text { steel, Fe-Ni-Cr alloys }\end{array}$ & $\begin{array}{l}\text { Immerse or swab for } \\
\text { few seconds to a } \\
\text { few minutes }\end{array}$ \\
\hline $\begin{array}{l}\text { Lepito's } \\
\text { Reagent }\end{array}$ & $\begin{array}{l}50 \mathrm{~mL} \text { acetic acid } \\
50 \mathrm{~mL} \text { nitric acid }\end{array}$ & $\begin{array}{l}\text { High temperature } \\
\text { steels }\end{array}$ & Swab \\
\hline $\begin{array}{l}\text { Marble's } \\
\text { Reagent }\end{array}$ & $\begin{array}{l}50 \mathrm{~mL} \text { distilled water } \\
50 \mathrm{~mL} \text { hydrochloric acid } \\
10 \mathrm{~g} \text { of copper sulfate }\end{array}$ & $\begin{array}{c}\text { Stainless steels, nickel } \\
\text { alloys }\end{array}$ & $\begin{array}{l}\text { Immerse or swab for } \\
\text { a few seconds }\end{array}$ \\
\hline $\begin{array}{l}\text { Murakami } \\
\text { Reagent }\end{array}$ & $\begin{array}{l}100 \mathrm{~mL} \text { distilled water } \\
10 \mathrm{~g} \mathrm{~K}_{3} \mathrm{Fe}(\mathrm{CN})_{8} \\
10 \mathrm{~g} \mathrm{NaOH} \text { or } \mathrm{KOH}\end{array}$ & $\begin{array}{l}\text { Wrought stainless } \\
\text { steel, tungsten alloys, } \\
\text { silver alloys, } \mathrm{SiC}, \mathrm{B}_{4} \mathrm{C}\end{array}$ & $\begin{array}{l}\text { Immerse or swab for } \\
\text { seconds to minutes }\end{array}$ \\
\hline Picral & $\begin{array}{l}100 \mathrm{~mL} \text { ethanol } \\
\text { 2-4 g Picric acid }\end{array}$ & $\begin{array}{c}\text { Iron and steel, tin } \\
\text { alloys }\end{array}$ & Seconds to minutes \\
\hline $\begin{array}{l}\text { Vilella's } \\
\text { Reagent }\end{array}$ & $\begin{array}{l}45 \mathrm{~mL} \text { glycerol } \\
15 \mathrm{~mL} \text { nitric acid } \\
30 \mathrm{~mL} \text { hydrochloric acid }\end{array}$ & $\begin{array}{l}\text { Stainless steels, carbon } \\
\text { steel, cast iron }\end{array}$ & Seconds to minutes \\
\hline
\end{tabular}


Table 5-8. Thermo-physical properties of elements at room temperature.

\begin{tabular}{cccccccc}
\hline Element & $\begin{array}{c}\text { Melting } \\
\text { Point } \\
\left({ }^{\circ} \mathrm{C}\right)\end{array}$ & $\begin{array}{c}\text { Boiling } \\
\text { Point } \\
\left({ }^{\circ} \mathrm{C}\right)\end{array}$ & $\begin{array}{c}\text { Density } \\
\left(\mathrm{g} / \mathrm{cm}^{3}\right)\end{array}$ & $\begin{array}{c}\text { Conductivi } \\
\text { ty }(\mathrm{W} / \mathrm{m} \\
\mathrm{K})\end{array}$ @STR & $\begin{array}{c}\text { Heat } \\
\text { Capacity } \\
(\mathrm{J} / \mathrm{mol} \mathrm{K})\end{array}$ & $\begin{array}{c}\text { Index of } \\
\text { Refraction }\end{array}$ & $\begin{array}{c}\text { Extinction } \\
\text { Coefficient }\end{array}$ \\
\hline $\mathrm{Hf}$ & $2,226.80$ & 4,602 & 13.31 & 23 & 25.64 & 1.39 & 1.19 \\
$\mathrm{Si}$ & 1,414 & 3,265 & 2.32 & 149 & 19.78 & 1.01 & 2.9 \\
$\mathrm{Fe}$ & 1,538 & 2,862 & 7.87 & 80.4 & 25.1 & 2.873 & 3.35 \\
$\mathrm{Cr}$ & 1,907 & 2,671 & 7.19 & 93.9 & 23.35 & 1.45 & 1.7 \\
\hline
\end{tabular}

Table 5-9. EDS quantification analysis on preliminary laser synthesis stage employing hafniumsilicon melted compounds.

\begin{tabular}{cccc}
\hline & Element & Weight \% & Atomic \% \\
\hline \multirow{3}{*}{ Point 1} & & & \\
& Si & 98.27 & 96.9 \\
& C & 1.31 & 3.03 \\
& Hf & 0.41 & 0.06 \\
& & & \\
Point 2 & Si & 91.35 & 97.94 \\
& Hf & 1.59 & 0.27 \\
& Sn & 7.06 & 1.79 \\
& & & \\
& & & 97.08 \\
& Pi & 86.46 & 1.59 \\
& Sn & 5.98 & 1.34 \\
\hline
\end{tabular}


Table 5-10. Chemical composition of cerium silicide

\begin{tabular}{ccc}
\hline Spot & Cerium (At. \%) & Silicon (At. \%) \\
\hline 1 & 47.07 & 52.93 \\
2 & 46.57 & 53.43 \\
3 & 6.71 & 93.29 \\
4 & 8.32 & 91.68 \\
5 & 36.36 & 63.64 \\
6 & 36.18 & 63.82 \\
7 & 35.86 & 64.14 \\
8 & 49.92 & 50.08 \\
9 & 95.33 & 4.67 \\
\hline
\end{tabular}

Table 5-11. EDS quantification analyses on zirconium-silicon melted compounds.

\begin{tabular}{ccc}
\hline Spot & Zirconium (At. \%) & Silicon (At. \%) \\
\hline 1 & 58.05 & 41.95 \\
2 & 57.04 & 42.96 \\
3 & 54.81 & 45.19 \\
4 & 60.75 & 39.25 \\
5 & 58.09 & 41.91 \\
6 & 59.12 & 40.88 \\
7 & 57.65 & 42.35 \\
8 & 56.53 & 43.47 \\
9 & 59.00 & 41.00 \\
10 & 58.98 & 41.02 \\
11 & 59.09 & 40.91 \\
12 & 84.16 & 15.84 \\
13 & 88.7 & 11.3 \\
14 & 82.72 & 17.28 \\
15 & 57.89 & 42.11 \\
16 & 58.27 & 41.73 \\
\hline
\end{tabular}


Table 5-12. EDS quantification analyses on hafnium-silicon melted compounds.

\begin{tabular}{ccc}
\hline Spot & Hafnium (At. \%) & Silicon (At. \%) \\
\hline 1 & 57.95 & 42.05 \\
2 & 56.13 & 43.87 \\
3 & 61.03 & 38.97 \\
4 & 54.07 & 45.93 \\
5 & 55.55 & 44.55 \\
6 & 44.82 & 55.18 \\
7 & 48.09 & 51.91 \\
8 & 49.94 & 50.06 \\
\hline
\end{tabular}


A

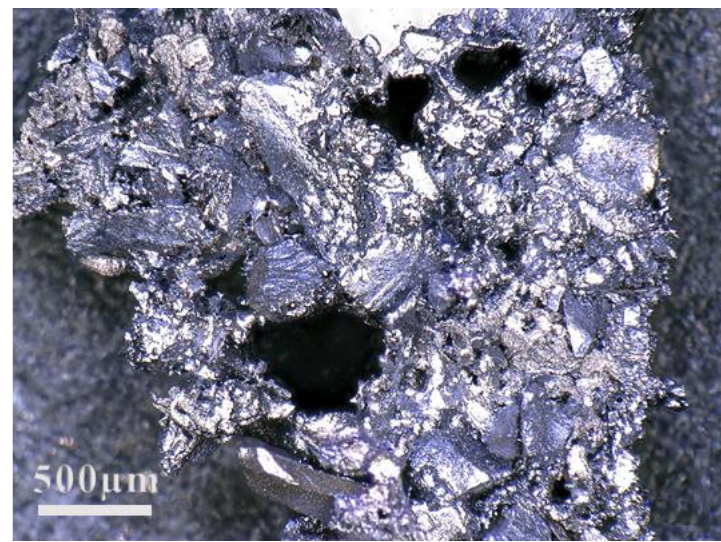

B

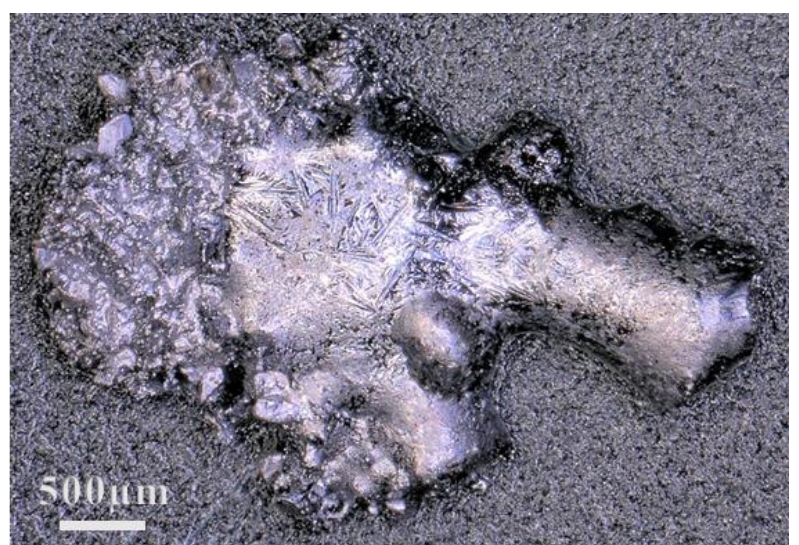

Figure 5-1. Optical microscopy images of preliminary experiments of the AMAFT process. A) Optical microscopy image of hafnium silicide after laser synthesis. B) Optical microscopy image of hafnium silicide revealing partial melting.

A
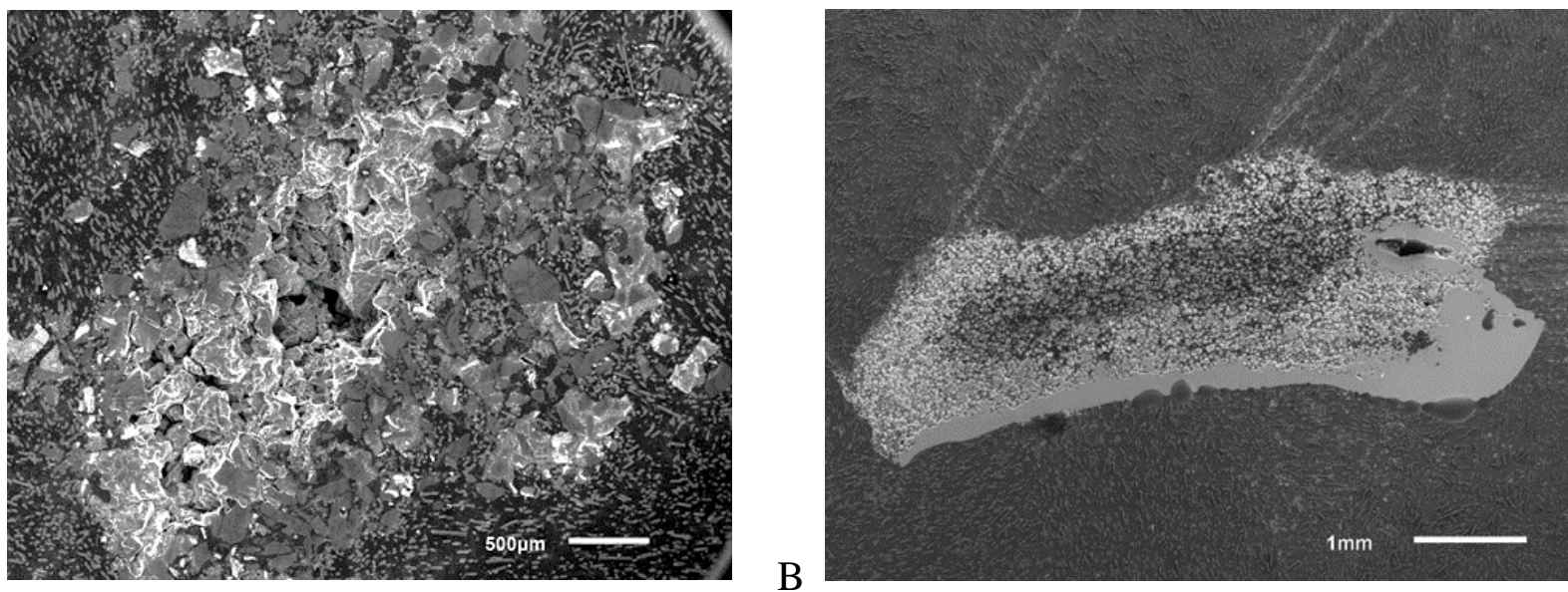

Figure 5-2. SEM micrographs of preliminary experiments of the AMAFT process. A) SEM image of hafnium silicide fabricated by laser synthesis. B) SEM image of a stainless steel sample fabricated by laser synthesis. 


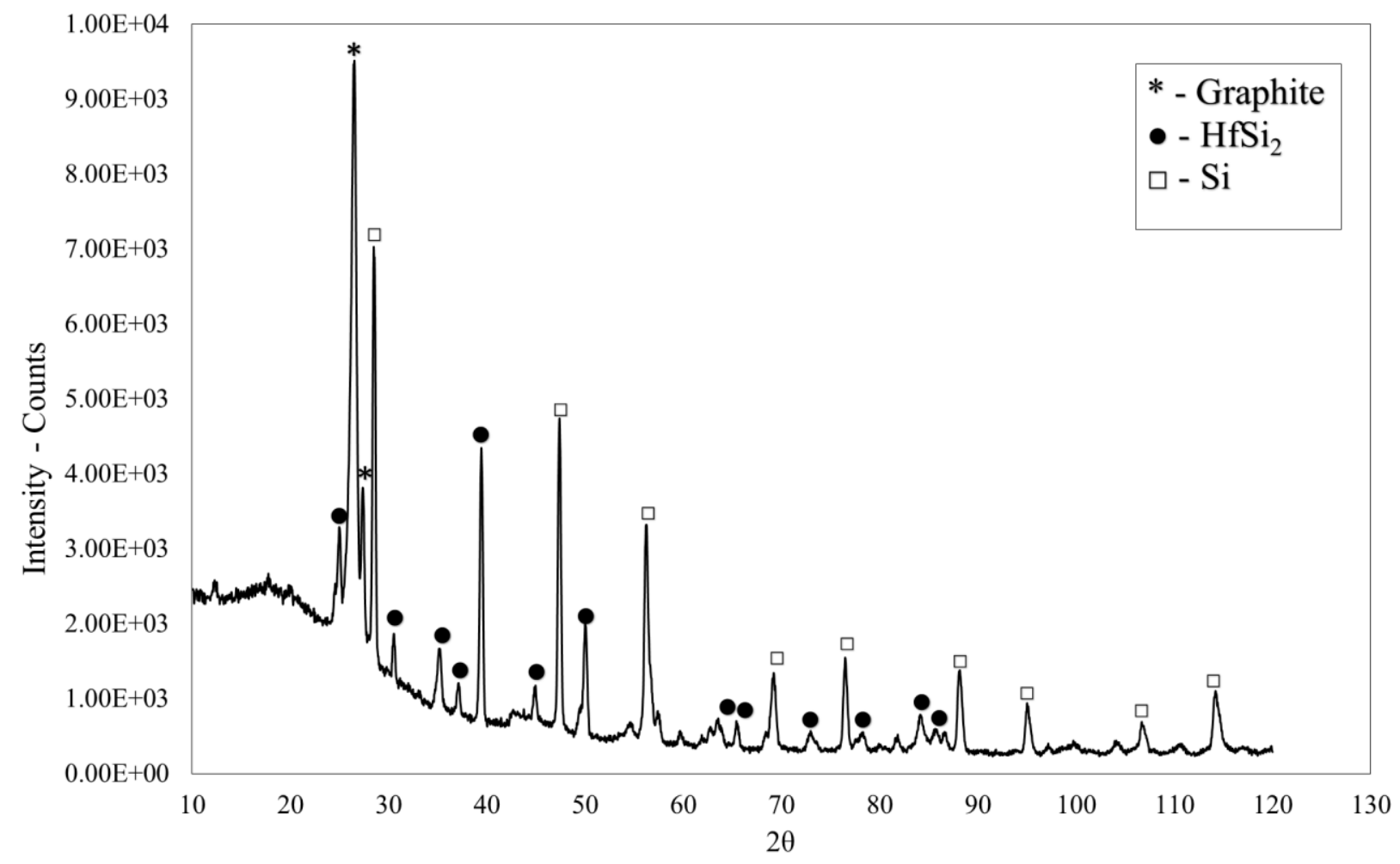

Figure 5-3. XRD pattern collected on a hafnium silicide compound.

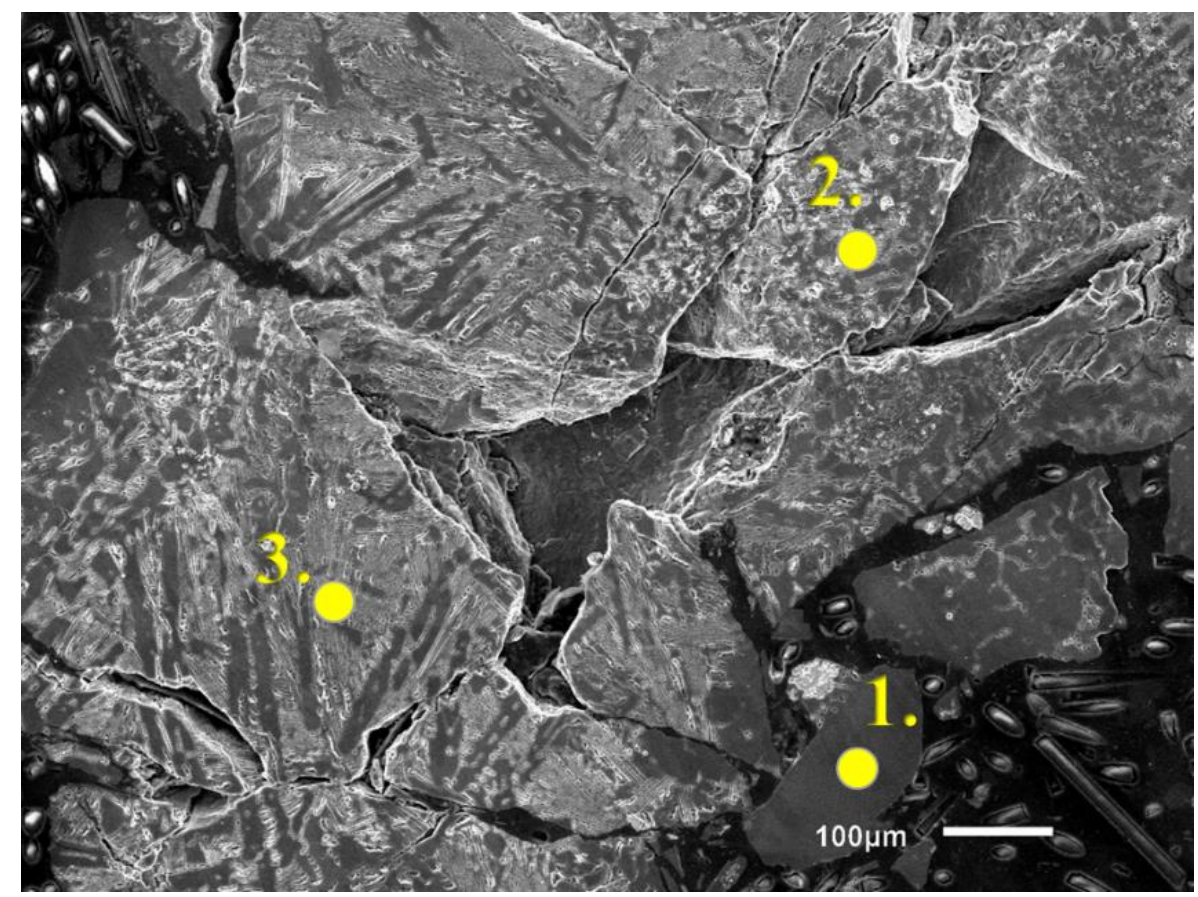

Figure 5-4. SEM micrograph displaying the morphology of a hafnium silicide compound from which EDS analyses were collected on different locations (Table 5-9). 

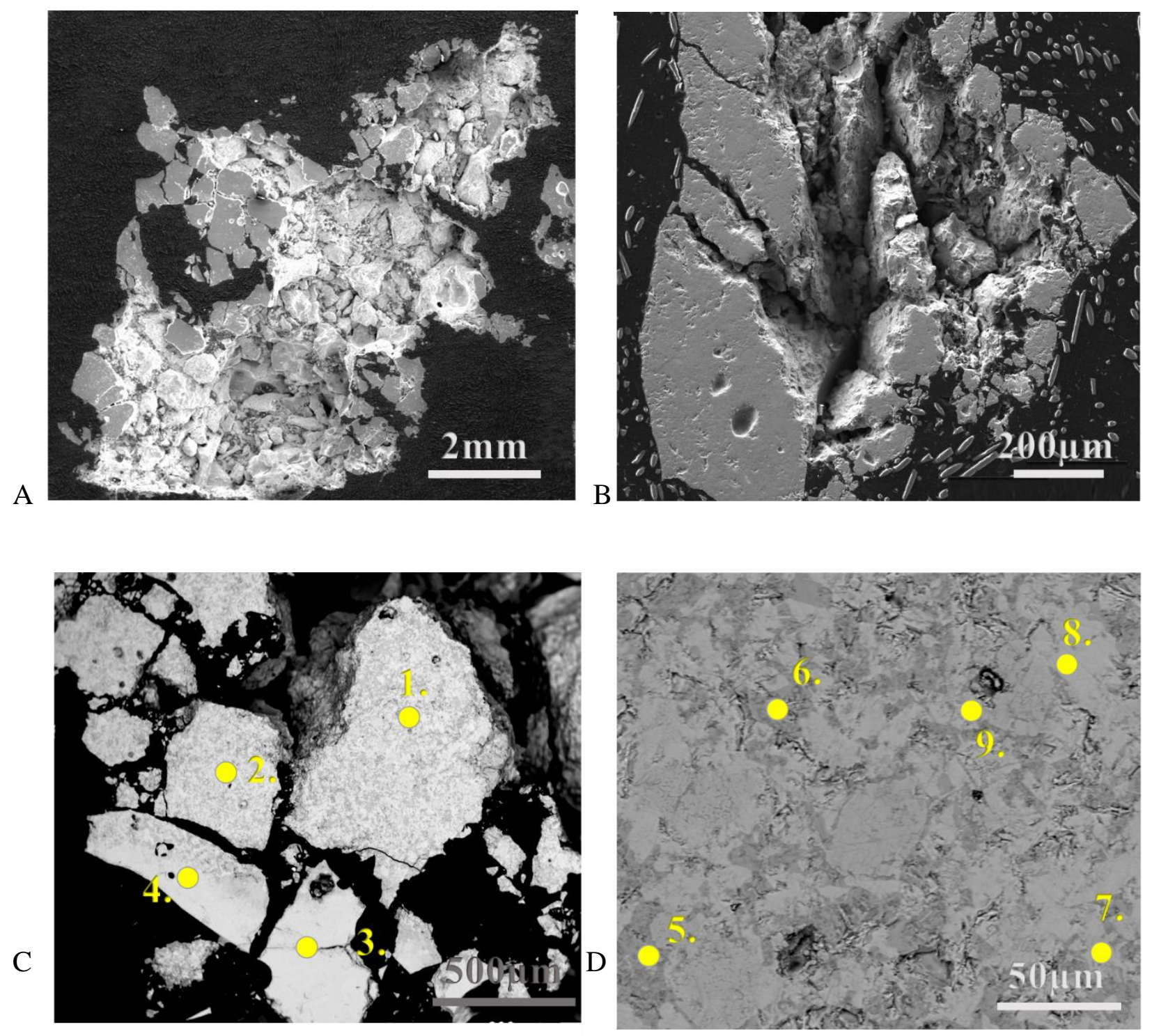

Figure 5-5. SEM micrographs displaying the microstructure of laser synthesized cerium-silicide. A) Partial melting of cerium-silicon powder samples. B) Voids generated during the laser synthesis. C) Back-scatter electron image displays the areas where EDS spots were analyzed. D) Morphology of cerium-silicide compound upon which EDS analyses were performed. 


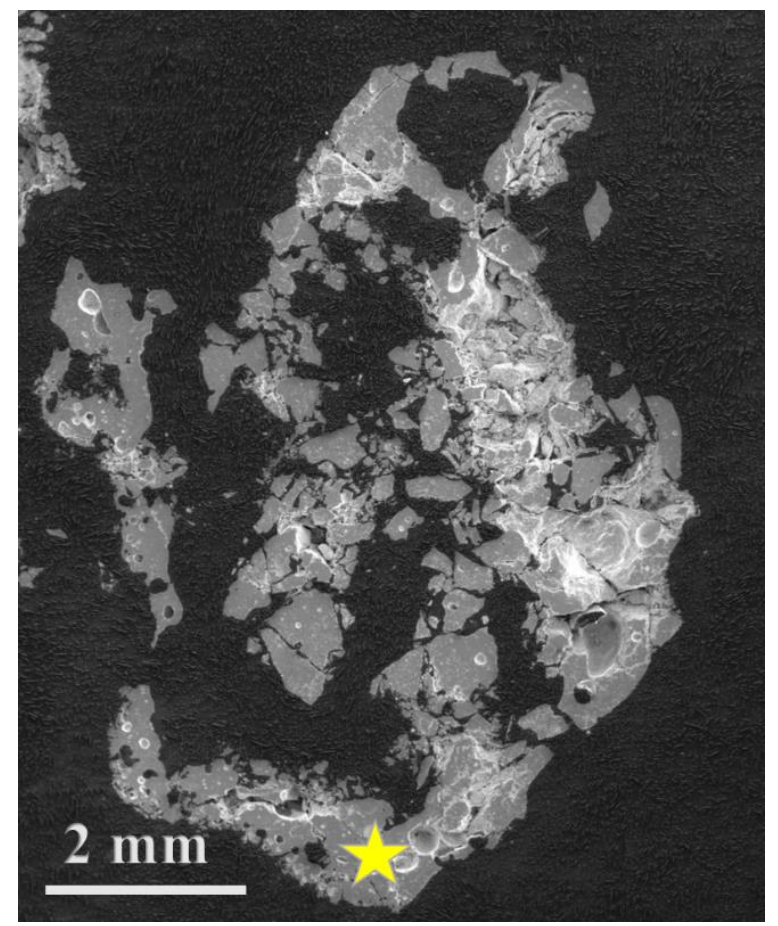

Figure 5-6. SEM micrograph displaying a spot on the cerium-silicon compound where XRD analysis was performed.

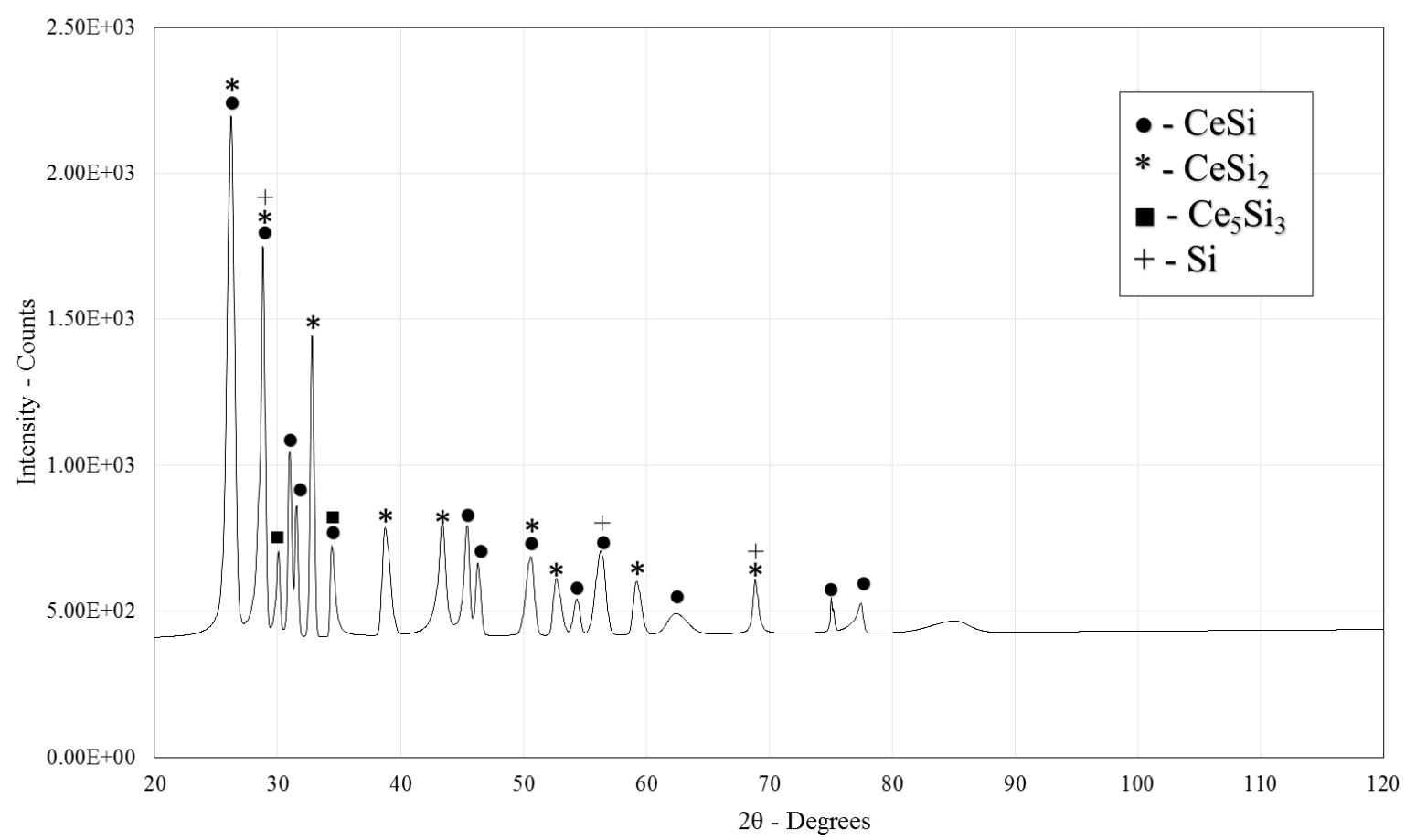

Figure 5-7. XRD pattern collected on cerium-silicon compound. 


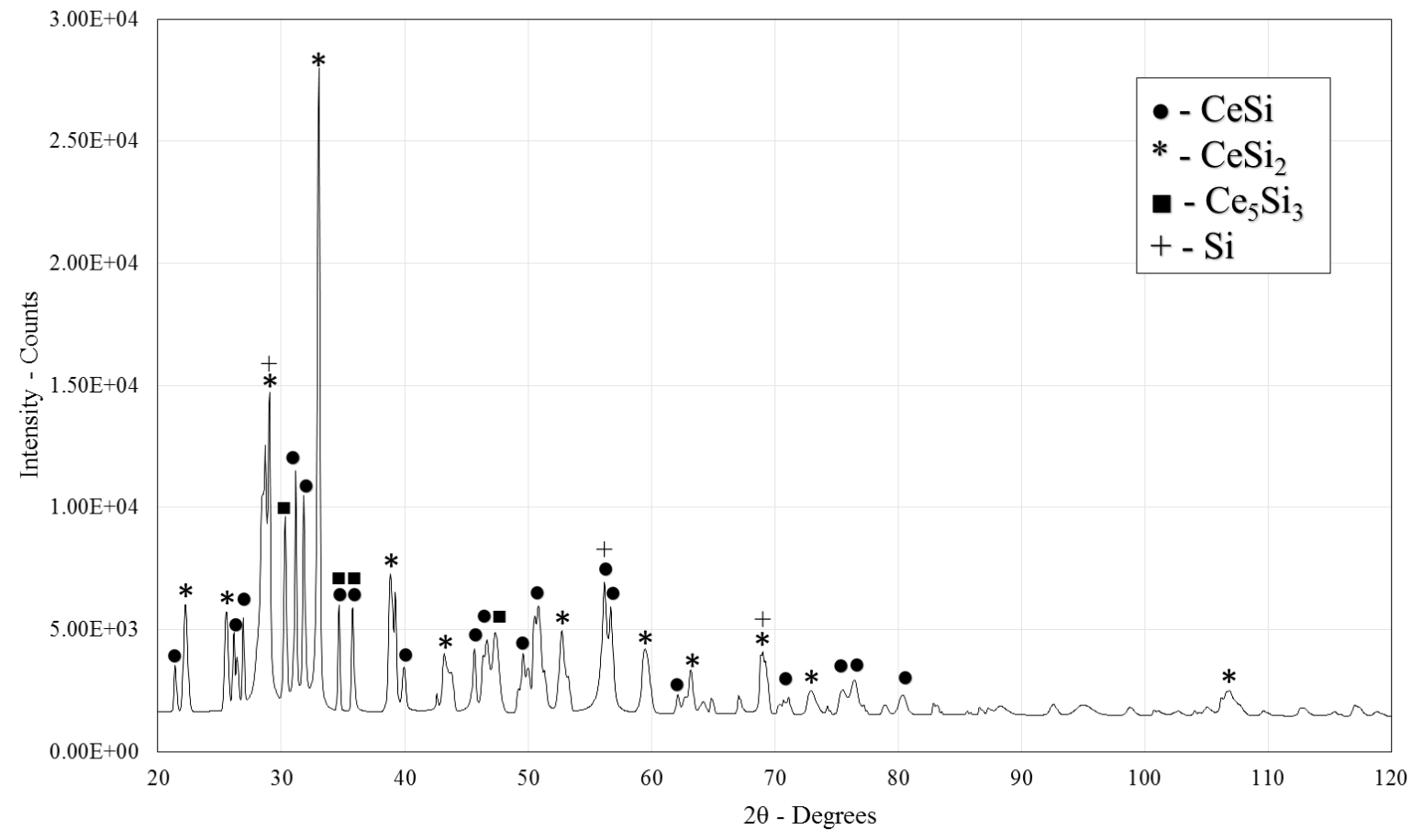

Figure 5-8. XRD pattern collected on cerium-silicon powder. 

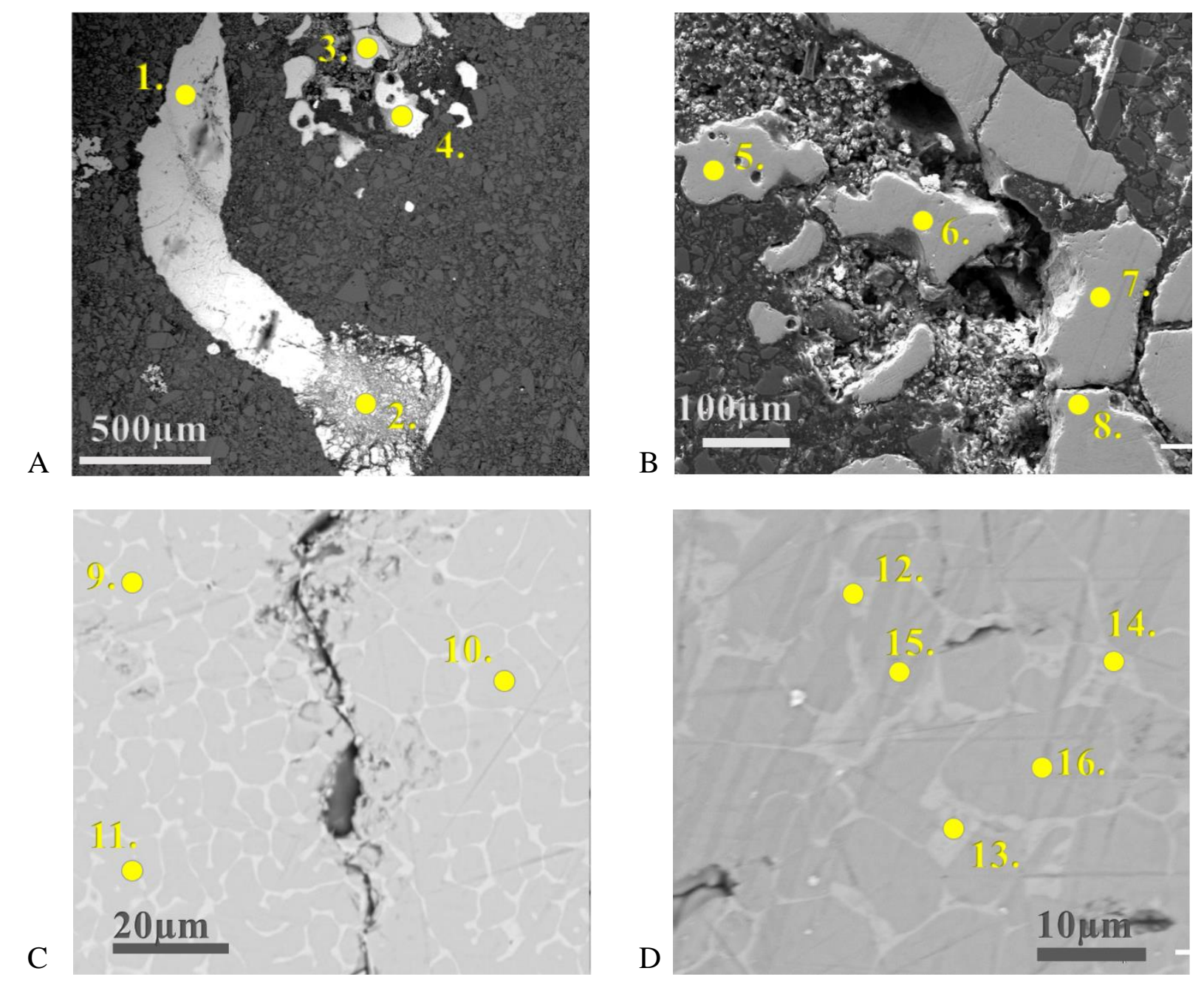

Figure 5-9. SEM images of zirconium silicide fabricated by laser synthesis. Spots indicate where EDS analyses were performed. A) SEM image displaying morphology of zirconium silicide. B) Secondary electron image displaying porosity and partial melting of the zirconium silicide compound. C) Back-scatter electron image display grain structure and formation of $\mathrm{Zr}_{3} \mathrm{Si}_{2}$. D) Back-scatter electron image displaying the grain boundary where high concentrations of $\mathrm{Zr}$ were found. 

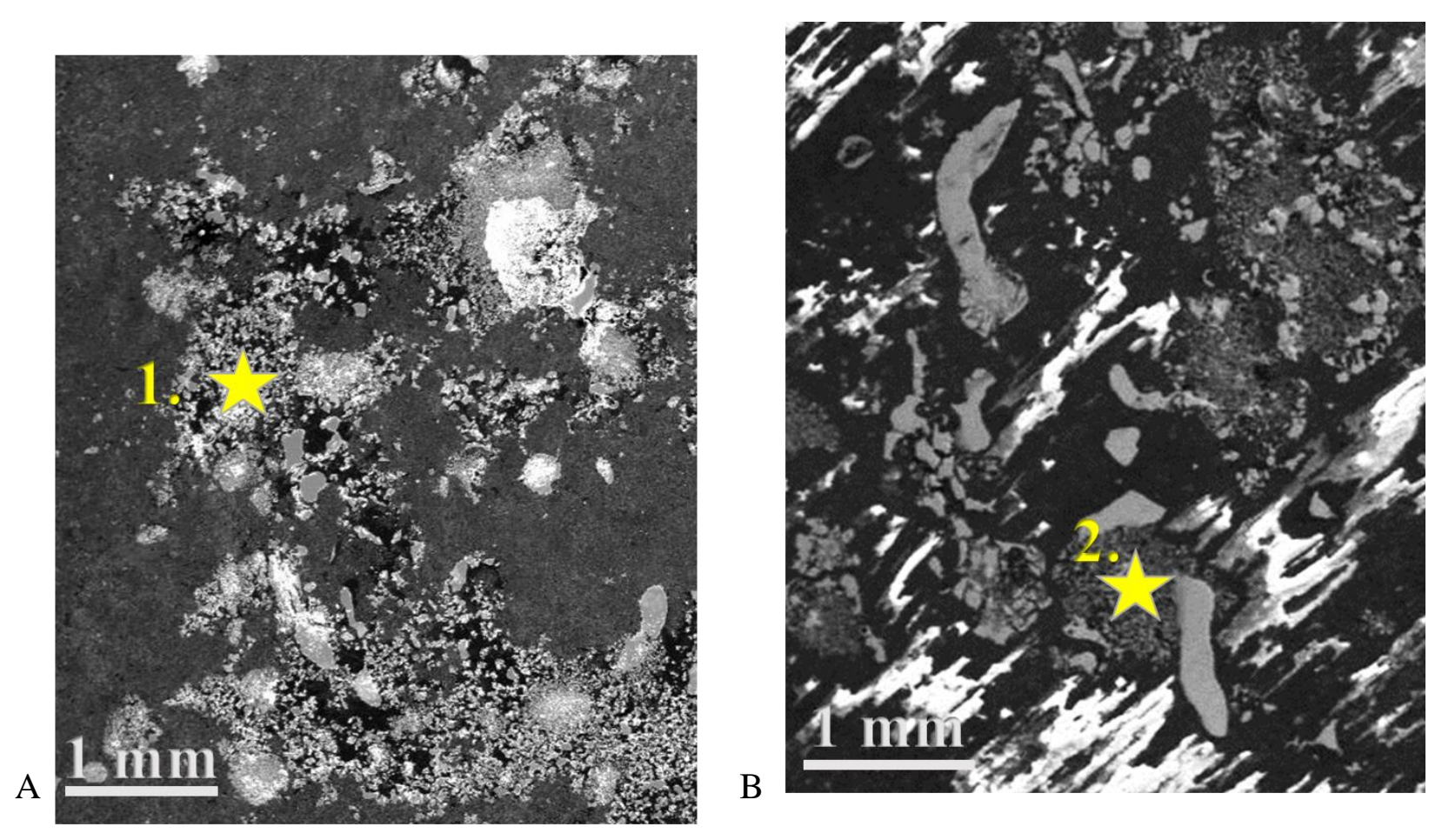

Figure 5-10. SEM micrographs of laser synthesized Zr-Si. A) SEM micrograph displaying where the XRD analysis was collected on Sample SZ-S1. B) SEM micrograph displaying where the XRD analysis was collected on Sample SZ-S2.

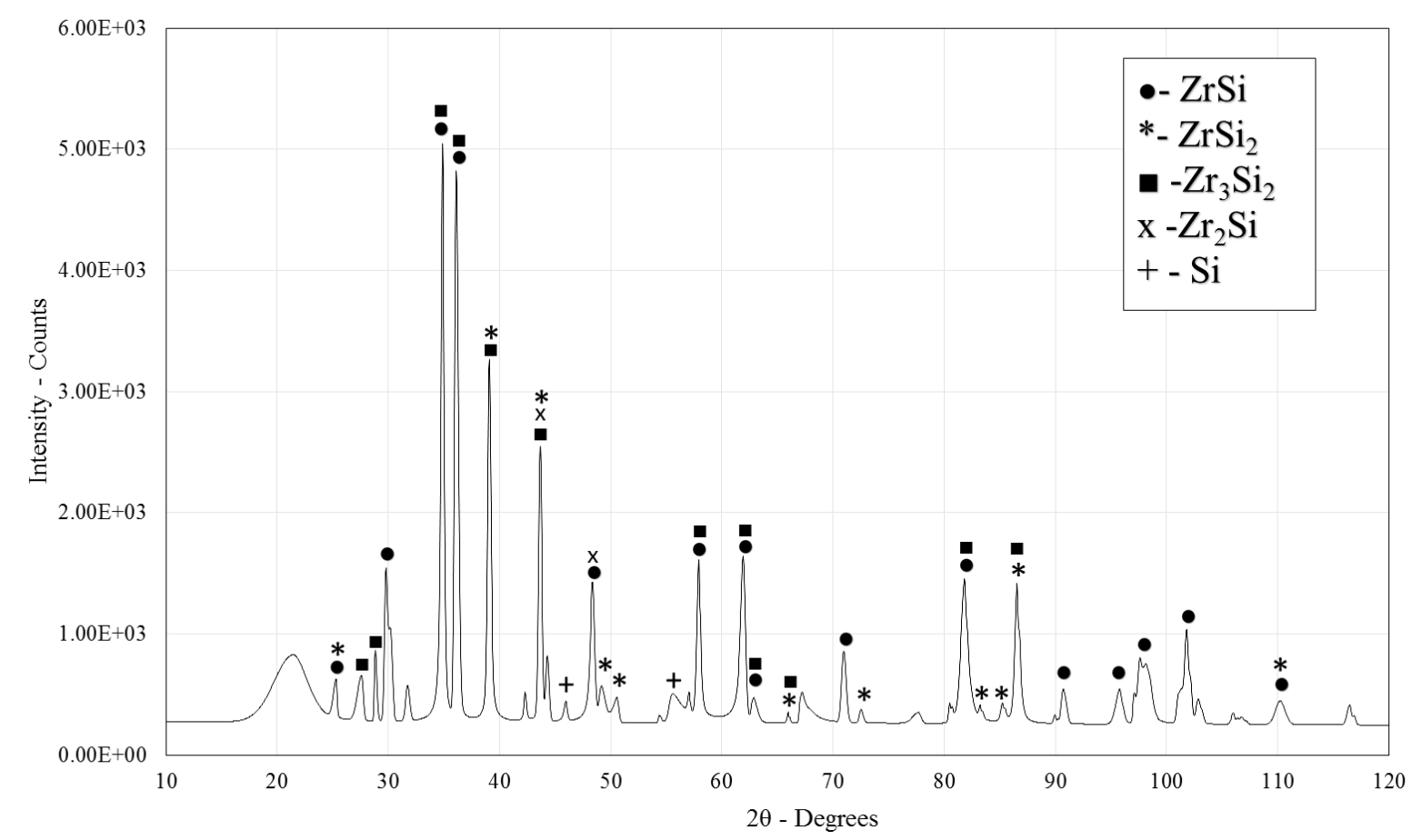

Figure 5-11. XRD pattern collected on Zr-Si Sample SZ-S1. 


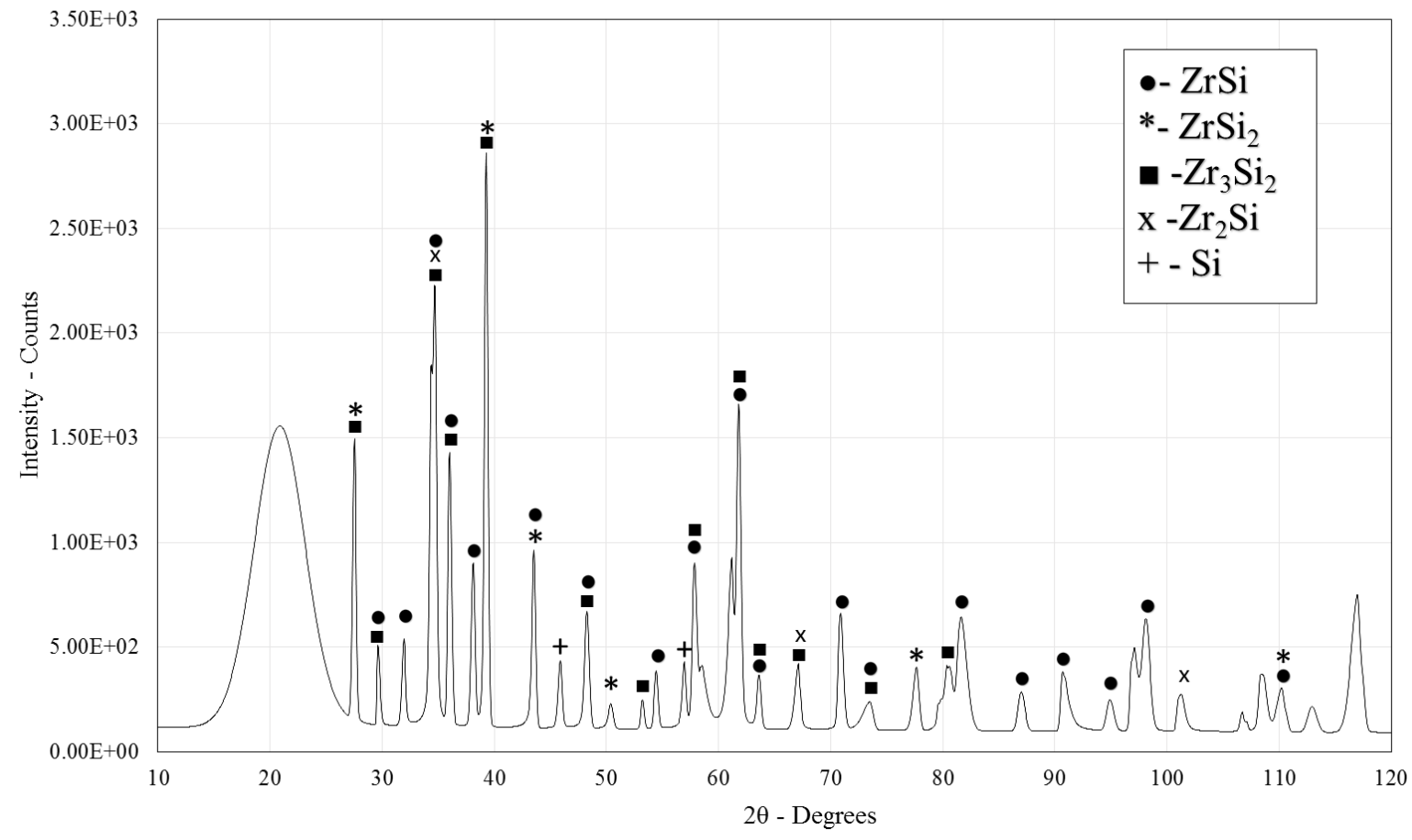

Figure 5-12. XRD pattern collected on Zr-Si Sample SZ-S2. 

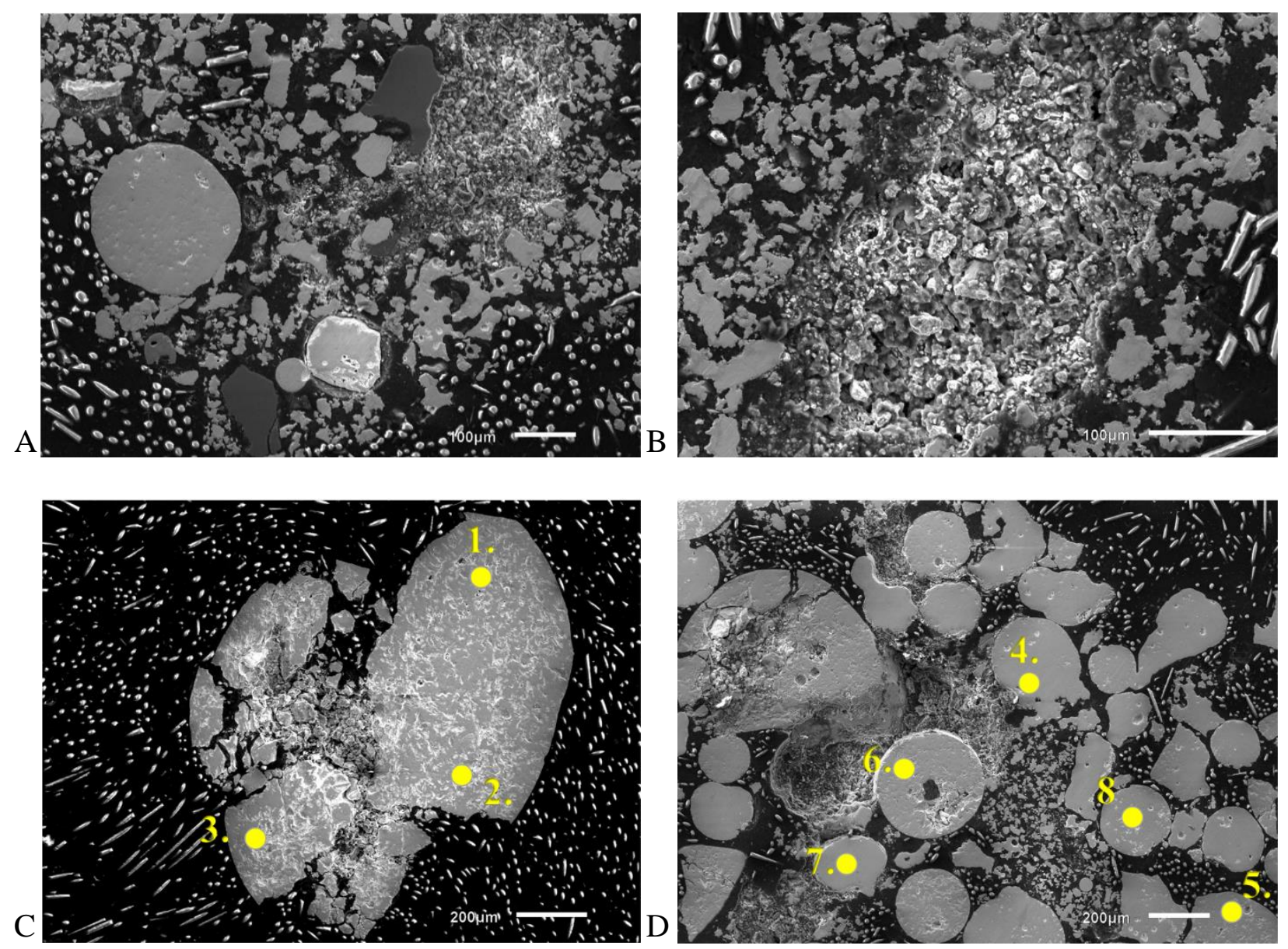

Figure 5-13. SEM micrographs of hafnium silicide fabricated by laser synthesis. A) SEM image displaying the morphology of a hafnium silicide sample. B) SEM micrograph displaying partial melting of hafnium silicide. C) SEM image displaying the areas where EDS spots were analyzed. D) Morphology of a hafnium-silicide compound upon which EDS analyses were performed. 


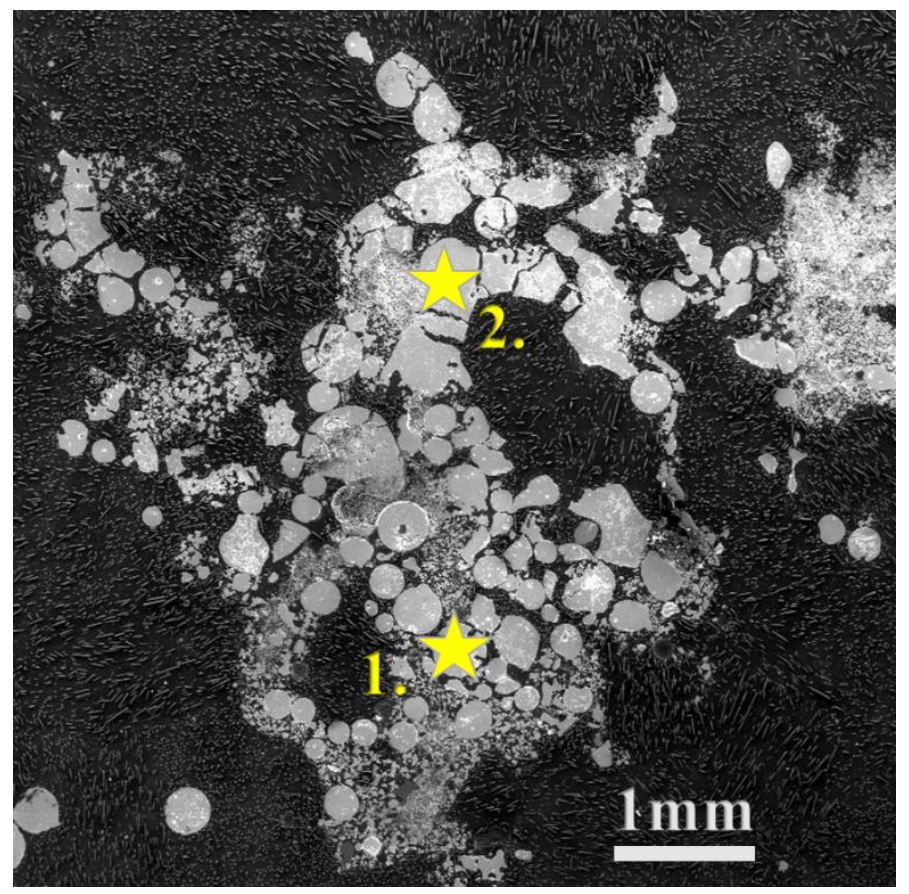

Figure 5-14. SEM micrograph displaying locations where XRD readings were collected on hafnium-silicide Sample HS-S1.

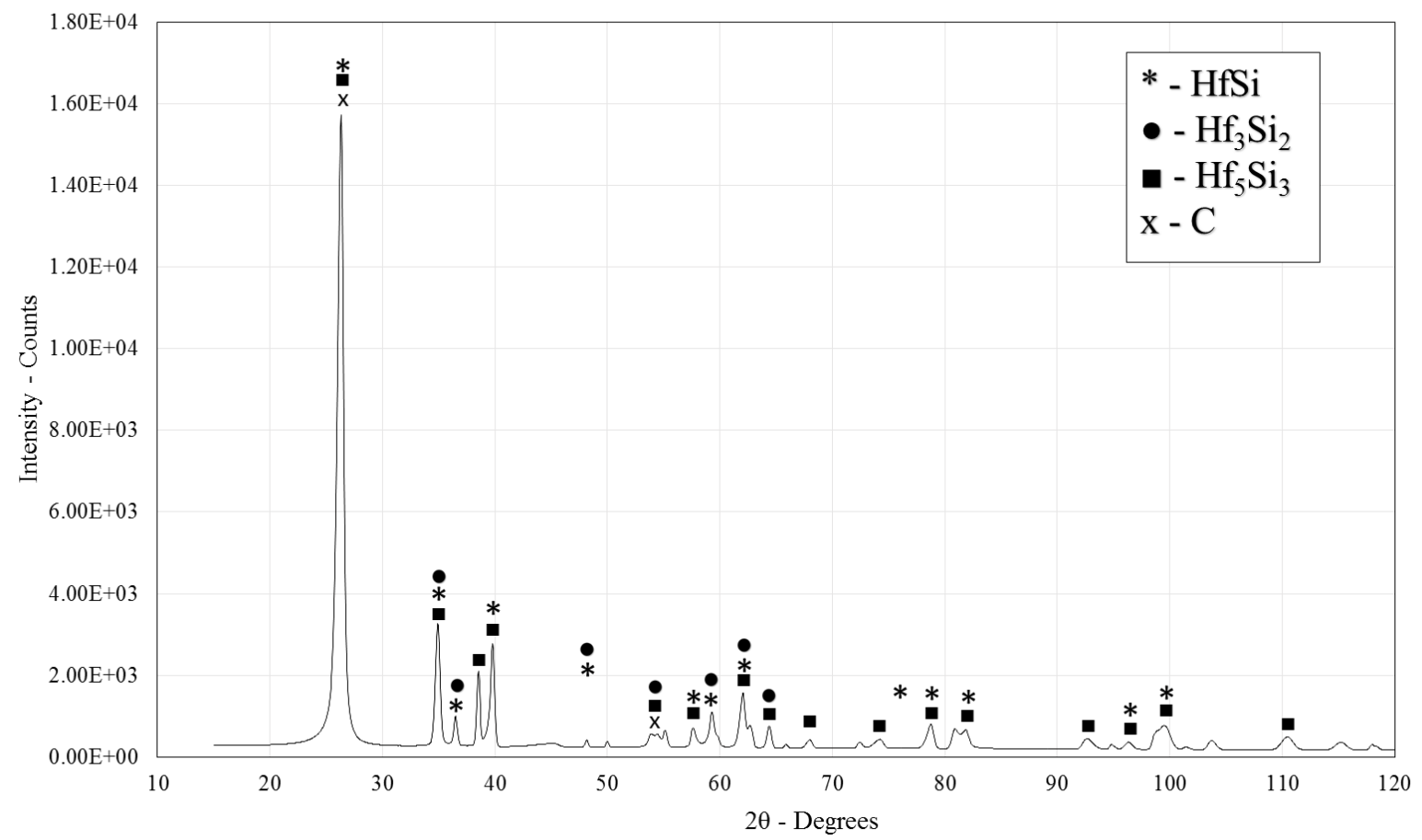

Figure 5-15. XRD analysis on laser synthesis of cerium-silicon compound (Spot 1 on Figure 5$14)$. 


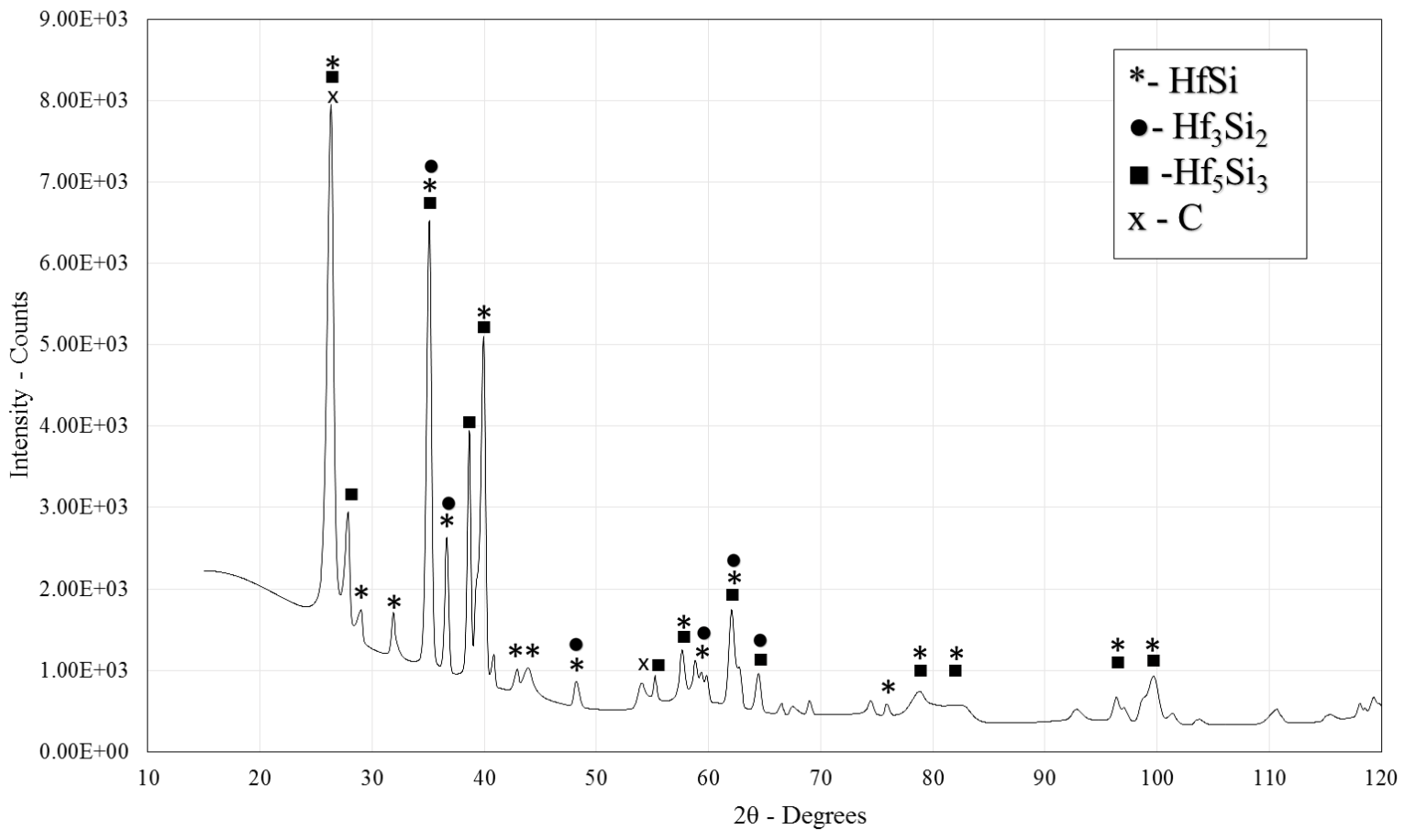

Figure 5-16. XRD analysis on laser synthesis of cerium-silicon compound (Spot 2 on Figure 514). 


\section{CHAPTER 6 \\ A PREDICTION OF THE FORMATION OF U $\mathrm{Si}_{2}$ BASED ON SURROGATE \\ CHARACTERIZATION RESULTS}

\section{Background}

The microstructure of the surrogate samples fabricated through the additive manufacturing process was characterized. Based on characterization data, predictions were formulated regarding microstructural properties of $\mathrm{U}_{3} \mathrm{Si}_{2}$ samples if prepared with similar process parameters. The microstructural analyses improved understanding of the effect of lasermaterial interaction on morphology and integrity. This has created a microstructural baseline for the next phase of uranium-based development work.

Characterization analyses including SEM, TEM, XRD, EDS and SAD were performed on $\mathrm{U}_{3} \mathrm{Si}_{2}$ fuel samples fabricated through a powder metallurgical approach (conventional sintering) developed at INL. A microstructural comparison of the additive manufactured surrogates and the $\mathrm{U}_{3} \mathrm{Si}_{2}$ fuel samples fabricated under the metallurgical approach was generated, as part of the scope Objective 3. The comparison has provided novel data to formulate predictions of the $\mathrm{U}_{3} \mathrm{Si}_{2}$ microstructure undergoing a laser synthesis. The characterization results have increased the understanding of the formation of surrogate silicide fuel prior to utilizing uranium-bearing compounds as part of development of this novel additive manufacturing methodology. The aforementioned studies were performed as a deliverable for Objective 3 (Chapter 1 pg. 20).

\section{Characterization of $\mathrm{U}_{3} \mathrm{Si}_{2}$ Powder Metallurgical Route}

\section{Electron Microscopy}

\section{Scanning electron microscopy and energy dispersive spectroscopy}

Scanning electron microscopy was performed on $\mathrm{U}_{3} \mathrm{Si}_{2}$ samples fabricated by a powder metallurgical approach developed at INL [14]. SEM analyses supported the evaluation of the samples' integrity and morphology. Back scatter electron microscopy enabled identification of 
the different phases present by means of mass contrasting along with chemical analysis.

Porosities were analyzed using SEM and quantified by means of digital image analysis according to the standards specified in ASTM E-1245[73]. Image $\mathrm{J}^{\mathrm{TM}}$ software was employed to evaluate the images and calculate the respective area fraction. Porosities among all samples appeared in a relatively low concentration, averaging $0.91 \% \pm 0.47$ of the evaluated areas. Such results are in agreement with the porosity fraction calculated in another study [14]. Figure 6-1 displays the different voids on the morphology of $\mathrm{U}_{3} \mathrm{Si}_{2}$ samples fabricated by a powder metallurgical approach. High porosity levels tend to hinder sample density, thus minimizing the porosities is crucial for optimum fuel performance.

Chemical analysis was performed on the samples by means of EDS. EDS incorporates a non-destructive technique that allows the identification of the elemental composition of the samples of interest. This technique is a semi-quantitative method and the results in this work were further analyzed and confirmed with X-ray diffraction and SAD analyses. The different phases found through the chemical analyses were in the fuel matrix and consisted of $\mathrm{U}_{3} \mathrm{Si}_{2}$ and USi as a secondary phase. This is in agreement with other studies on the U-Si phases present on $\mathrm{U}_{3} \mathrm{Si}_{2}$ fuel [16][19][74]. Figure 6-2 displays an electron backscatter micrograph displaying the different areas where the chemical analysis confirmed the presence of $\mathrm{U}_{3} \mathrm{Si}_{2}$, which forms the fuel matrix. The results from the EDS analysis are displayed in Table 6-1. The average atomic concentration of uranium and silicon was found to be $61.77 \% \pm 5.79$ and $38.21 \% \pm 5.79$ respectively, which is in accordance to the U-Si phase diagram for the specific composition of $\mathrm{U}_{3} \mathrm{Si}_{2}$ [17]. In addition, the USi phase was revealed by the chemical analysis. Figure 6-3 shows electron back-scatter images where quantification analyses was performed on different areas. The USi phase was identified by EDS and later confirmed by XRD. Due to the weaker mass 
contrast, the USi phase is displayed in a darker gray on the back-scatter micrographs. Table 6-2 displays the quantification of the chemical analysis where the average concentrations of uranium and silicon were $50.15 \% \pm 1.21$ and $49.84 \pm 1.21$, respectively, thus, implying the presence of the USi phase. Findings of $\mathrm{U}_{3} \mathrm{Si}$ were also made by means of EDS and later confirmed by XRD. Figure 6-4 displays electron back-scatter micrographs where chemical analyses revealed the presence of the $\mathrm{U}_{3} \mathrm{Si}$ phase. Due to weak mass contrast, the areas containing higher concentrations of $\mathrm{U}_{3} \mathrm{Si}$ display a very dark tonality when compared with the rest of the fuel. Table 6-3 shows the quantification results on the chemical analyses, where the average concentrations of uranium and silicon were $74.24 \% \pm 1.37$ and $25.76 \% \pm 1.37$, respectively, suggesting the presence of $\mathrm{U}_{3} \mathrm{Si}$ in the compound. Other research works indicate that $\mathrm{U}_{3} \mathrm{Si}$ is not a desirable phase as it is assumed to become amorphous when irradiated, resulting in breakaway and swelling [76]. In addition, the silicide $\left(\mathrm{U}_{3} \mathrm{Si}_{2}\right)$ fuel is a mixture of $\mathrm{U}_{3} \mathrm{Si}_{2}, \mathrm{USi}$, and sporadic amounts of $\mathrm{U}_{3} \mathrm{Si}$. In practice, it is almost impossible to fabricate a perfect homogenous fuel [76].

\section{Transmission Electron Microscopy}

A total of four $\mathrm{U}_{3} \mathrm{Si}_{2}$ samples were prepared by a dual-beam focused ion beam (Quanta 3D FEG) system. A platinum coating was added to the prepared samples to bring protection to the oxide surface during cutting. The lamellae were fabricated at dimensions of: $10 \times 15 \times 0.2 \mu \mathrm{m}$. After cutting, the four lamellae were inserted into copper holders prior to TEM examination. Figure 65 displays the four lamellae fabricated by FIB. Some lamellae display holes in the middle area that were originated during the cutting and refining process. In the bottom part the attachment to the copper holder can be observed. Samples A2 and D4 have a higher concentration of silicon than Samples C1 and C2. Complimentary details on the silicon content can be found on the upcoming section: "Analysis of High Silicon Content on $\mathrm{U}_{3} \mathrm{Si}_{2}$ fuel." To perform the TEM 
analyses a $300 \mathrm{kV}$ Tecnai TF30 scanning electron microscope (STEM) equipped with an EDS system was employed to evaluate the samples. Figure 6-6 displays micrographs of the analyzed $\mathrm{U}_{3} \mathrm{Si}_{2}$ samples. The different locations where the EDS analyses were performed have been numbered on each of the micrographs. Table 6-4 displays the quantification of the chemical analyses performed on each of the samples. Figure 6-6 (a) displays Sample A2, where the EDS analyses suggested the presence of $\mathrm{UO}_{2}$ and USi on Spots 1 and 2 respectively, surrounded by a $\mathrm{U}_{3} \mathrm{Si}_{2}$ matrix as displayed by Spots 3 and 4. Figure 6-6 (b) displays sample D4, where EDS suggests the presence of $\mathrm{UO}_{2}$ on a $\mathrm{U}_{3} \mathrm{Si}_{2}$ matrix displayed on Spots 5 and 6 respectively. Additionally, a large hole originated during the sample refining stage can be observed in the middle of the lamellae. Figure 6-6 (c) displays Sample C1 where the EDS suggest the presence of $\mathrm{UO}_{2}$ on a grain targeted as Spot 7. Figure 6-6 (d) shows a grain on sample C2, where the EDS analysis suggests the presence of $\mathrm{UO}_{2}$. STEM analyses assisted to identify minuscule $\mathrm{UO}_{2}$ and USi grains coexisting in the $\mathrm{U}_{3} \mathrm{Si}_{2}$ lamellae.

The grains found have a higher mass contrast when compared to the $\mathrm{U}_{3} \mathrm{Si}_{2}$ matrix. Additionally, the phases found in the TEM results are consistent with XRD, and SAD analyses mentioned in this dissertation. The presence of oxygen is attributed to surface oxide of the sample powders as well as potential $\mathrm{O}_{2}$ impurities found in the argon filled glovebox where the fabrication took place. Oxidation of metallic uranium aids in the formation of silicon-rich USi phases while employing uranium and silicon powders in a stoichiometric ratio to form $\mathrm{U}_{3} \mathrm{Si}_{2}$. The phases found in the TEM analyses performed in this research project were in close agreement with other literature evaluating silicide $\left(\mathrm{U}_{3} \mathrm{Si}_{2}\right)$ fuel [17][19][75]. Future work will evaluate the interphase of USi and $\mathrm{UO}_{2}$ grains found in the fuel matrix. 


\section{X-Ray Diffraction}

$\mathrm{X}$-ray diffraction (XRD) spectroscopy was performed on the $\mathrm{U}_{3} \mathrm{Si}_{2}$ fuel samples in order to evaluate the different phases present. The XRD analyses was performed after mounting the samples in resin holders for characterization. A Rigaku X-ray diffractometer (XRD) with $\mathrm{Cu} \mathrm{K \alpha}$ radiation was employed to evaluate the samples. The scanning step was set to $0.04^{\circ}$ at an approximate scanning speed of $1 \%$ min. Figure 6-7 illustrates SEM micrographs of the $\mathrm{U}_{3} \mathrm{Si}_{2}$ fuel samples where the diffraction patterns were acquired. For this study, the diffraction patterns were collected at the inner center of each sample. The XRD analyses confirmed that the fuel matrix was predominantly composed of the $\mathrm{U}_{3} \mathrm{Si}_{2}$ phase. The secondary phases confirmed by the XRD analyses included: $\mathrm{USi}, \mathrm{UO}_{2}, \mathrm{U}_{3} \mathrm{Si}$, and metallic uranium. The reference files are from 01-0812241( $\left(\mathrm{U}_{3} \mathrm{Si}_{2}\right), 00-041-0845\left(\mathrm{U}_{3} \mathrm{Si}\right), 00-027-0928$ (USi), 00-041-1422 $\left(\mathrm{UO}_{2}\right)$ and 00-011-0628 (uranium). Some of the secondary phases were in lesser proportions, thus their intensities in the diffraction peaks were superimposed by the $\mathrm{U}_{3} \mathrm{Si}_{2}$ phase which had a higher concentration. Figures 6-8 and 6-9 display the XRD results for Sample A2 and D4, respectively, where USi, $\mathrm{UO}_{2}, \mathrm{U}_{3} \mathrm{Si}$, and metallic uranium were found. Figure 6-10 shows the XRD pattern for Sample C where $\mathrm{UO}_{2}, \mathrm{U}_{3} \mathrm{Si}$, and metallic uranium were present. The XRD results are in close agreement with Harp et al. [14] which the primary phase of the compound was $\mathrm{U}_{3} \mathrm{Si}_{2}$. Additionally, the presence of $\mathrm{USi}, \mathrm{UO}_{2}$, and metallic uranium match the results acquired in other XRD works [14][16] [17][19]. 


\section{Thermal Diffusivity}

The thermal properties of nuclear fuels are essential for understanding and predicting fuel performance during reactor operation. The thermal properties of the fuel should be analyzed in both fresh and irradiated samples, as these properties are crucial for controlling the heat transfer from the fuel into the surrounding coolant. The thermal properties can influence the fuel pin peak operating power, margin to melting, fuel pin design, and overall fuel performance [77].

The thermal properties of $\mathrm{U}_{3} \mathrm{Si}_{2}$ samples were conducted with a temperature range of 25 to $1,000^{\circ} \mathrm{C}$, which mirrors the approximate temperature range during reactor operation. The data was collected by means of a Netzsch Laser Flash Analyzer (LFA427). Figure 6-11 displays the thermal diffusivity of $\mathrm{U}_{3} \mathrm{Si}_{2}$ fuel samples as a function of temperature. The diffusivity measurements are compared with the data acquired by White et al.[16] on a similar material with a higher density. The data collected at INL displays a slightly decreased diffusivity when compared with values of other literature. Such a decrease may be attributed to the lower density of the samples fabricated or the multiple secondary phase impurities found in the fuel.

The thermal transport of $\mathrm{U}_{3} \mathrm{Si}_{2}$ is highly influenced by phonon scattering and electronic contributions and can be severely affected by the presence of defects in the microstructure. The $\mathrm{U}_{3} \mathrm{Si}_{2}$ manufactured under the powder metallurgical approach developed at INL is not a pure phase. The matrix is composed by the $\mathrm{U}_{3} \mathrm{Si}_{2}$ phase but different secondary phases from the U-Si system have been identified by means of XRD, chemical analyses, and SAD. The analyses performed suggest that in addition to U-Si compounds, the oxide phase $\mathrm{UO}_{2}$ is present. The presence of an oxide phase makes a significant impact altering the overall thermal properties of the bulk due to the significantly lower conductivity of the $\mathrm{UO}_{2}$ compound when compared to any of the U-Si phases. The oxide phases have lower and thermal diffusivities than those of $\mathrm{U}_{3} \mathrm{Si}_{2}$. 
Therefore, the presence of oxide phases leads to a decrease in both mass density and thermal conductivity [77].

\section{Effect of High Si-Content on $\mathrm{U}_{3} \mathrm{Si}_{2}$ Fuel Microstructure}

\section{Background}

After the March 2011 events at the Fukushima Daiichi Nuclear Plant in Japan, improving the accident tolerance, safety, and reliability of nuclear fuel has become a topic of research interest [78]. The Fuel Cycle \& Development Program (FCRD) is exploring new accident tolerant fuel (ATF) concepts along with industry partners, national laboratories and research universities. A more highly uranium dense fuel like $\mathrm{U}_{3} \mathrm{Si}_{2}$ will allow the same number of fissile $\mathrm{U}^{235}$ atoms to be accommodated in a smaller volume of material. This property can lead to large savings in both enrichment and fuel manufacturing costs, which are crucial aspects to evaluate in the selection of an accident-tolerant fuel [15]. $\mathrm{U}_{3} \mathrm{Si}_{2}\left(12.2 \mathrm{~g} / \mathrm{cm}^{3}\right)[14]$ is among the fuels with the highest density when compared with $\mathrm{UAl}_{2}\left(8.1 \mathrm{~g} / \mathrm{cm}^{3}\right)[24], \mathrm{U}_{3} \mathrm{O}_{8}\left(8.3 \mathrm{~g} / \mathrm{cm}^{3}\right)[24], \mathrm{UO}_{2}(10.97$ $\left.\mathrm{g} / \mathrm{cm}^{3}\right)[24]$, and USi $\left(11.0 \mathrm{~g} / \mathrm{cm}^{3}\right)[24]$.

The comparatively improved thermal conductivity of $\mathrm{U}_{3} \mathrm{Si}_{2}$ can decrease the thermal gradients and generate lower centerline temperatures during fuel performance. Studies have demonstrated that from $400-1,000^{\circ} \mathrm{C}$, the thermal conductivity of $\mathrm{U}_{3} \mathrm{Si}_{2}$ drastically exceeds that of $\mathrm{UO}_{2}[16]$. Also, from a safety perspective, improvements in thermal conductivity can potentially slow down the rise rate of the core temperature during accident conditions. Additionally, the $\mathrm{U}_{3} \mathrm{Si}_{2}$ irradiation stability of the fuel is promising, with fission gases forming small uniform bubbles with minimal coalescence, which limits fuel swelling and ultimately offers greater stability during high burnups [26]. The constant fission gas bubble spacing along with uniform bubble size in $\mathrm{U}_{3} \mathrm{Si}_{2}$ justifies its excellent swelling behavior. Its stable bubble morphology has been observed up to 80\% swelling in HEU at a burnup of over 60\% [26]. 
The methods to produce $\mathrm{U}_{3} \mathrm{Si}_{2}$ fuel are high-energy ball milling (HEBM)[18], centrifugal atomization [20] and powder metallurgy [14] processes. The scope of this research work is to understand the effect of the silicon content on the microstructure of $\mathrm{U}_{3} \mathrm{Si}_{2}$ fuel fabricated by means of a conventional powder metallurgical process.

\section{Experimental}

Silicide fuel $\left(\mathrm{U}_{3} \mathrm{Si}_{2}\right)$ was fabricated using a powder metallurgical method. The process has proven to produce $\mathrm{U}_{3} \mathrm{Si}_{2}$ with densities above $95 \%$ theoretical density, as well as an optimized high phase purity. As part of the goal to develop an industrially scalable process to produce $\mathrm{U}_{3} \mathrm{Si}_{2}$ pellets, a laboratory-scale development was performed. Due to the highly pyrophoric behavior of the powders and an effort to avoid oxidation, the fabrication process took place in a glovebox with an argon atmosphere with oxygen content below $10 \mathrm{ppm}$. The samples were manufactured following the procedures developed at Idaho National Laboratory [14].

One sample (C) was prepared with uranium and silicon powders in stoichiometric proportions. (92.7 wt\% and $7.3 \mathrm{wt} \%$, respectively). Two additional samples (A2 and D4) were fabricated with higher amounts of silicon (U and Si powders at $92.5 \mathrm{wt} \%$ and $7.5 \mathrm{wt} \%$, respectively) to account for the silicon loss expected during the arc melting stage. Furthermore, studies suggest the inclusion of extra silicon aids in minimizing the formation of metallic uranium and $\mathrm{U}_{3} \mathrm{Si}$, which compromise the most U-rich phases in the U-Si system [1]. Table 6-5 displays some of the manufacturing parameters employed during the production of the $\mathrm{U}_{3} \mathrm{Si}_{2}$ samples.

The production of silicide fuels generally results in relative amounts of the various phases or distinguishable crystalline entities present in the U-Si alloy at different Si concentrations. From previous studies, it may be considered essentially impossible to produce an alloy at the exact stoichiometry of the compound and of such atomistic homogeneity that only a pure phase 
is present [1]. A minor, but finite, amount of the phase to the left or the right on the phase diagram [17] of the compound of interest can be found in the microstructure. Furthermore, to increase the challenge of producing a pure phase, the presence of impurities, which are inevitable, can allow the formation of other phases that may or may not be noticeable under microscopy, depending on the size and the magnification employed. The impurities found can be expected to appear in a solid state, sometimes within the crystalline lattices, in each of the phases present. The aim of this work is to further explore the presence of these different phases and/or impurities using various characterization techniques with a range of resolutions.

\section{Results and Discussion}

The samples' morphologies, microstructures, and chemical analyses were examined using a JEOL scanning electron microscope (model JSM-6610-LV) coupled with energy dispersive X-ray spectroscopy (EDS). X-ray diffraction spectroscopy (XRD) was performed using a Rigaku Smartlab X-ray diffractometer. The transmission electron microscopy (TEM) samples were prepared by a dual-beam Quanta 3D focused ion beam instrument. Scanning transmission electron microscopy (STEM) and conventional TEM analyses were conducted on an FEI Tecnai F30 microscope operated at $300 \mathrm{kV}$. STEM images were obtained using a camera focal length of $80 \mathrm{~mm}$. Chemical analyses on TEM samples were carried out using the $\mathrm{EDAX}^{\mathrm{TM}}$ energy dispersive spectroscopy (EDS) system. Gatan Digital micrograph and TIA ${ }^{\mathrm{TM}}$ (TEM imaging and analysis) software were used for post-processing of TEM data. The simulations of diffraction patterns were carried out using JEMS ${ }^{\mathrm{TM}}$ software.

Scanning electron microscopy was performed to evaluate the microstructural details in all three $\mathrm{U}_{3} \mathrm{Si}_{2}$ samples. Figure 6-12 shows backscattered SEM images of conventional (C) and high silicon (A2 and D4) samples. Mass contrast imaging, along with chemical analysis allowed identification of the $\mathrm{U}_{3} \mathrm{Si}_{2}$ matrix, porosities, and secondary phases in all three $\mathrm{U}_{3} \mathrm{Si}_{2}$ samples. 
The predominant secondary phases found in the samples were USi and $\mathrm{UO}_{2}$. Sample A2 displays an acicular morphology of USi as displayed in Figure 6-12 (a). Samples D4 and C displayed a flakey morphology of USi as observed from SEM images on Figure 6-12 (b) and Figure 6-12 (c) respectively. Higher proportions of porosity were found on Sample D4 as displayed by Figure 6-12 (b) and confirmed later by digital image analysis (Table 6-6).

Digital image analysis was further employed to quantify the phase proportions of all samples (Table 6-6). Employing a SEM backscatter electron detector along with the guidelines specified on the ASTM E-1245 [73], the area fractions of the uranium dioxide and uranium silicon-rich phases were estimated. These results also indicate that higher concentrations of silicon allow the formation of secondary phases of the U-Si system, predominantly USi. Although no significant statistical differences in porosity between samples with high and low silicon content are measured, there is an indication that the porosity levels between the two high silicon samples are different. At this point, no reason for this could be determined from the available fabrication parameters. It was determined that the $\mathrm{U}_{3} \mathrm{Si}_{2}$ matrix proportion was above $80 \%$ for all three samples. Larger proportions of the secondary phase (USi) were found on samples (specifically A2 and D4) with higher silicon content due to silicon interaction with uranium metal during fabrication. Based on the U-Si phase diagram [17] it is suggested that the higher concentrations of silicon react, forming a USi phase during sintering at $1,550{ }^{\circ} \mathrm{C}$. The phase quantification results are in close agreement with the work performed in [14].

The XRD analyses on the $\mathrm{U}_{3} \mathrm{Si}_{2}$ samples confirmed the presence of secondary phases. Three different areas were evaluated on each sample and only the spots closer to the edge of the pellet are shown in Figure 6-13. According to the XRD analyses, the secondary phases found in the samples were $\mathrm{U}_{3} \mathrm{Si}, \mathrm{USi}$, and $\mathrm{UO}_{2}$. This is in agreement with the phases found through 
electron microscopy of the present work and also has been reported elsewhere [17][19]. On the samples with higher silicon content, peaks displayed the presence of silicon in its elemental state, showing incomplete reactions.

The presence of an oxide phase makes a major impact in the fuel by altering its thermal properties. This occurs due to a significant lower thermal conductivity of the $\mathrm{UO}_{2}$ compound when compared to any U-Si phases. It is confirmed that the higher silicon content tends to react with uranium metal and form USi and $\mathrm{U}_{3} \mathrm{Si}$ during sintering, which are the two phases surrounding $\mathrm{U}_{3} \mathrm{Si}_{2}$ in the $\mathrm{U}-\mathrm{Si}$ phase diagram. For $\mathrm{U}_{3} \mathrm{Si}$ the swelling due to fission gas is different from that of $\mathrm{U}_{3} \mathrm{Si}_{2}$. The swelling rate for $\mathrm{U}_{3} \mathrm{Si}$ is drastically higher resulting in fuel pillowing. The origin of such swelling behavior is attributed to its gas bubble morphology which displays interlinkage generating a premature amorphous morphology in the compound. For USi, its lower density suggests that a large majority of the uranium is fissioned at moderately high fission rates [24]. Due to the aforementioned effects, the presence of secondary phases on the $\mathrm{U}_{3} \mathrm{Si}_{2}$ fuel can be detrimental during fuel performance.

\section{TEM}

The structural identification of various phases formed within $\mathrm{U}_{3} \mathrm{Si}_{2}$ matrix has been carried out by TEM selected area diffraction. The STEM image of the conventional Sample C in Figure 6-14 (a) shows a grain possessing weaker mass contrast compared with that of the rest of the FIB lamellae. The SAD corresponding to the matrix is shown in Figure 6-14 (b). The SAD pattern confirms the tetragonal structure of the $\mathrm{U}_{3} \mathrm{Si}_{2}$ phase (Space group $\mathrm{P} 4 / \mathrm{mbm}$ ). The high amount of oxygen in the darker grain compared with other parts of the lamellae, as revealed by EDS, attributes weaker mass contrast to the grain. The structure of this grain was identified as cubic $\mathrm{UO}_{2}$ (Space group Fm $\overline{3} \mathrm{~m}$ ) based on the SAD pattern shown in Figure 6-14 (c). The high- 
resolution (HR)TEM image along the [001] zone axis in Figure 6-14 (d) shows the atomic structure of $\mathrm{UO}_{2}$.

Figure 6-15 (a) shows a STEM image of the high-silicon Sample A2. The morphology of the grain with weaker mass contrast has been shown by a bright field image in the inset. The matrix was confirmed to be tetragonal $\mathrm{U}_{3} \mathrm{Si}_{2}$ phase by the $\mathrm{SAD}$ pattern as shown in Figure 6-15 (b). Although the SAD in Figure 6-15 (c) confirmed the same tetragonal structure of the grain, the EDS results confirm that the weaker mass contrast of the grain is attributed to high silicon content when compared with that of the matrix. Hence, the grain was identified to be USi based upon the chemical and structural analyses. Figure 6-15 (d) shows the HRTEM image of the USi grain along the [211] zone axis. On Sample A2, the high concentration of silicon allowed the formation of USi compound, which is in agreement with the phase diagram [17] and the XRD results. The fuel matrix was confirmed to be $\mathrm{U}_{3} \mathrm{Si}_{2}$ by both XRD and $\mathrm{SAD}$. The phases found in this analysis are in agreement with the TEM results reported in [75]. In the TEM and SAD examinations not all the secondary phases were found, due to the smaller volume of the TEM specimen. Future work will include additional analyses of interfaces of phases found in $\mathrm{U}_{3} \mathrm{Si}_{2}$, to confirm the $\mathrm{U}_{3} \mathrm{Si}$ phases identified by the XRD analysis.

\section{Findings}

Two $\mathrm{U}_{3} \mathrm{Si}_{2}$ samples were prepared using a conventional fabrication process, with a higher silicon content (7.5 wt $\%)$ to account for the element loss along arc melting. A third sample was prepared with a silicon content of $7.3 \mathrm{wt} \%$. The higher concentrations of silicon allowed the formation of secondary phases, including $\mathrm{USi}, \mathrm{U}_{3} \mathrm{Si}, \mathrm{UO}_{2}$, and silicon in its elemental state (since it was left unreacted). The aforementioned phases were confirmed by XRD and electron microscopy. 
It is confirmed that the higher silicon content tends to react with uranium metal and form USi and $\mathrm{U}_{3} \mathrm{Si}$ during sintering, which are the two phases surrounding $\mathrm{U}_{3} \mathrm{Si}_{2}$ in the U-Si phase diagram. Only one of the higher silicon content samples, namely A2 displayed an increased amount of the USi phase as compared with Sample C. The formation of $\mathrm{UO}_{2}$ was attributed to oxygen, most probably present while manufacturing the uranium and silicon powders.

Prevention of oxygen exposure from uranium metal and silicon is highly suggested in order to minimize the formation of the $\mathrm{UO}_{2}$ compound. Research efforts are being made on manufacturing a pure $\mathrm{U}_{3} \mathrm{Si}_{2}$ phase leading to reduced fuel swelling, which brings stability in high burnups during reactor operation. The impact of these findings and the prediction to a laser based melting synthesis, is not directly related as the energy transfer rates are potentially different. However it is an input value to consider in conjunction with the evaporation rate of $\mathrm{Si}$ in the laser chamber and complete AMAFT process. 


\section{A Microstructural Comparison}

After fabricating surrogate silicide fuel by means of a laser synthesis, characterization analyses were performed. The characterization results assisted in understanding the interaction between materials and the laser source along the fabrication process. According to the EDS and XRD analyses performed, the successful formation of silicide surrogate fuel was accomplished with zirconium and hafnium in the experimental process. The microstructure of the laser synthesized $\mathrm{Zr}_{3} \mathrm{Si}_{2}$ is displayed in Figure 6-16 (a) and (b). In Figure 6-16 (a) porosities along with cracks can be observed and their formation is attributed to the rapid cooling generated during the uneven laser energy distribution. This uneven laser distribution is mainly due to the current bench experimental set up and can be prevented during the optimization and scale up process. In Figure 6-16 (b) the formation of $\mathrm{Zr}_{3} \mathrm{Si}_{2}$ can be observed with grain formation displaying a flaky morphology and high concentrations of zirconium present in the grain boundaries. Additionally, different phases were present as confirmed by XRD and EDS analyses (Figure 5-9 (d) and Figure 5-11).

When comparing the microstructure of $\mathrm{Zr}_{3} \mathrm{Si}_{2}$ with that of $\mathrm{U}_{3} \mathrm{Si}_{2}$ fabricated by means of a powder metallurgical process (Figure 6-16 (c)), both display an inhomogeneous morphology with the presence of secondary phases from their respective binary phase diagrams (U-Si and Zr$\mathrm{Si})$. The presence of oxide phases $\left(\mathrm{UO}_{2}\right)$ in the $\mathrm{U}_{3} \mathrm{Si}_{2}$ samples is assumed to occur during the conventional sintering stage under a vacuum. Nonetheless, no oxide formation was present on the zirconium silicide samples due to the presence of an inert argon atmosphere during the laser synthesis and due to the specific design approach of the AMAFT process. The AMAFT process mitigates the presence of oxide phases on the fabricated compounds. The enclosed chamber under an argon atmosphere isolates the system and prevents oxygen and other compounds from taking part in the reaction. Additionally, the alloying of the compounds occurs in only a few 
seconds, generating a shortage of time for oxide phases to form. Laser synthesis proved to be a feasible methodology to fabricate $\mathrm{Hf}_{3} \mathrm{Si}_{2}$. Figure 6-17 (a) displays the morphology of a laser synthesized sample of $\mathrm{Hf}_{3} \mathrm{Si}_{2}$. The upper section presents partial melting of the compound, generating an inhomogeneous surface finish due to the uneven heat distribution from the laser source. As mentioned previously for the $\mathrm{Zr}-\mathrm{Si}$ experimentation, this can be prevented by process optimization and scale up equipment changes. From the selected surrogate materials, hafnium had the highest melting point of $2,226.85^{\circ} \mathrm{C}$ [42]. In order to melt hafnium a high laser power output was employed. The laser energy beam generated an uneven heat distribution, which led to non-uniform cooling rates producing a coarse and inhomogeneous surface. The microstructure of $\mathrm{Hf}_{3} \mathrm{Si}_{2}$ is displayed in Figure 6-17 (b) where grain formation and different phases of the Hf-Si system can be observed. Due to the weaker mass contrast, the darker area is represented by $\mathrm{Hf}_{3} \mathrm{Si}_{2}$ and the lighter by $\mathrm{Hf}_{5} \mathrm{Si}_{3}$, the phases were later confirmed by XRD (Figure 5-15 and 516).

The laser synthesis was performed under an argon atmosphere and both, XRD and EDS analyses did not find any oxide phases present in the evaluated samples. A comparison between the microstructure of $\mathrm{Hf}_{3} \mathrm{Si}_{2}$ that of $\mathrm{U}_{3} \mathrm{Si}_{2}$ fabricated by a powder metallurgical method (Figure 617 (c)), showed that both compounds displayed an inhomogeneous morphology with the presence of secondary phases and the porosity content was in higher proportions on the $\mathrm{U}_{3} \mathrm{Si}_{2}$ compound, attributed to the high axial pressure generated during the powder compaction stage (green pellet pressing). Further work may incorporate the implementation of the laser parameters in order to improve the heat distribution to regulate the temperature gradients during sintering.

The microstructural comparison among $\mathrm{Zr}_{3} \mathrm{Si}_{2}, \mathrm{Hf}_{3} \mathrm{Si}_{2}$ and $\mathrm{U}_{3} \mathrm{Si}_{2}$ has set the basis for future experimentation with uranium compounds. The current research work aimed to 
demonstrate the feasibility of forming surrogate silicide fuel $\left(\mathrm{Zr}_{3} \mathrm{Si}_{2}\right.$ and $\left.\mathrm{Hf}_{3} \mathrm{Si}_{2}\right)$ employing a laser energy source. If performed under the same process parameters, the formation of a $\mathrm{U}_{3} \mathrm{Si}_{2}$ compound from uranium and silicon powders may be achievable. A laser synthesis of $\mathrm{U}_{3} \mathrm{Si}_{2}$ will validate the proof of concept of fabricating accident tolerant fuel by means of an additive manufacturing process.

\section{Prediction of $\mathrm{U}_{3} \mathrm{Si}_{2}$ Microstructure During a Laser Synthesis}

Laser irradiation on materials can be a complex subject involving optical, electromagnetic, thermodynamic, and mechanical changes on the microstructure of the material. The deposition of laser energy into a system may generate a rise in temperature, gasification and ionization [10]. When materials are subject to laser irradiation the incident laser energy will be absorbed, thus, generating an abrupt rise in temperature and causing material expansion along with thermal stresses. When the stress exceeds a certain threshold, the material may fracture or plastically deform. The thermal effects in laser ablation include melting, vaporization, boiling, and phase explosion, while the mechanical response may involve deformation and resultant stress in the microstructure [10].

Predictions can be made on the microstructure of $\mathrm{U}_{3} \mathrm{Si}_{2}$ while evaluating the thermodynamic properties and phase binary diagrams of the selected surrogate materials. The laser synthesis of uranium surrogates was performed and the characterization results were discussed in Chapter 5. Comparisons of the evaluated microstructure, through XRD and chemical analyses of the surrogate materials, along with phase diagrams and thermal properties of surrogates, educated predictions can be made on the formation of the $\mathrm{U}_{3} \mathrm{Si}_{2}$ compound.

The microstructural analysis on the synthesis of zirconium as a surrogate material displayed a fully molten phase, which suggests the occurrence of grain growth during solidification. The chemical analyses on the sample revealed the formation of $\mathrm{Zr}_{3} \mathrm{Si}_{2}$, which was 
later confirmed by XRD patterns. Figure 5-9 (c) (Chapter 5) displays the microstructure of $\mathrm{Zr}_{3} \mathrm{Si}_{2}$ fabricated under a laser synthesis. In addition, the experiments in Chapter 5 proved the formation of the surrogate compound $\mathrm{Hf}_{3} \mathrm{Si}_{2}$ by means of a laser synthesis (Figure 5-13). The experiments producing $\mathrm{Zr}_{3} \mathrm{Si}_{2}$ and $\mathrm{Hf}_{3} \mathrm{Si}_{2}$ not only validated the concept of fabricating surrogate nuclear fuel by means of a laser synthesis but also set the ground base for executing experiments with uranium compounds.

The $\mathrm{Zr}$-Si phase diagram [41] also suggests that the formation of $\mathrm{Zr}_{3} \mathrm{Si}_{2}$ occurs with 5660 atomic $\%$ zirconium, at temperatures around $1,880{ }^{\circ} \mathrm{C}$. While observing the phase diagram of the U-Si system, it is clear that the formation of $\mathrm{U}_{3} \mathrm{Si}_{2}$ occurs from 50 - 60 atomic $\%$ uranium. Which provides a larger margin for the formation of the $\mathrm{U}_{3} \mathrm{Si}_{2}$ compound at an approximate temperature of $1,540^{\circ} \mathrm{C}$ during the laser synthesis. From a thermodynamic standpoint, $\mathrm{U}_{3} \mathrm{Si}_{2}$ has the highest enthalpy of formation (-33.86 KJ/mol [79] ) when compared with $\mathrm{Ce}_{3} \mathrm{Si}_{2}(-60.9$ $\mathrm{KJ} / \mathrm{mol}, \mathrm{Zr}_{3} \mathrm{Si}_{2}(-384.56 \mathrm{KJ} / \mathrm{mol}[6])$ and $\mathrm{Hf}_{3} \mathrm{Si}_{2}(-400 \mathrm{KJ} / \mathrm{mol}$ [42]), suggesting it has the least exothermic reaction of the three surrogates. When comparing $\mathrm{Ce}_{3} \mathrm{Si}_{2}, \mathrm{Zr}_{3} \mathrm{Si}_{2}, \mathrm{Hf}_{3} \mathrm{Si}_{2}$, and $\mathrm{U}_{3} \mathrm{Si}_{2}$, they all conserve the same tetragonal crystal structure. Moreover, the Ce-Si, $\mathrm{Zr}-\mathrm{Si}$, and $\mathrm{Hf}-\mathrm{Si}$ binary phase diagrams share many similarities including multiple intermediate phases and various eutectic points. Also, $\mathrm{U}_{3} \mathrm{Si}_{2}$ has a relatively low Gibbs free energy $(-180,121 \mathrm{KJ} / \mathrm{mol}$ [79]) when compared with that of $\mathrm{Ce}_{3} \mathrm{Si}_{2}\left(-11 .{ }^{\circ} \mathrm{C}[6]\right)$, suggesting that a feasible and spontaneous reaction can occur. Further research efforts are being made to produce a highly pure phase of $\mathrm{U}_{3} \mathrm{Si}_{2}$ at quantification levels of $80 \%+$ present in the compound. While employing a powder metallurgical process, quantification analyses demonstrated that the $\mathrm{U}_{3} \mathrm{Si}_{2}$ phase was above the targeted $80 \%$ phase purity (See Table 6-6) [43]. Additionally the phase proportion was calculated for AMAFT fabricated $\mathrm{Hf}_{3} \mathrm{Si}_{2}$ and $\mathrm{Zr}_{3} \mathrm{Si}_{2}$, with quantification results of $84 \%$ and $86 \%$, 
respectively. Along the experimental work both the powder metallurgical method along with the AMAFT process were capable of fabricating surrogates and fuel compounds with a high purity phase. Therefore, it can be predicted that a potential high purity phase can be achieved while fabricating $\mathrm{U}_{3} \mathrm{Si}_{2}$ under the AMAFT process.

The likelihood of forming $\mathrm{U}_{3} \mathrm{Si}_{2}$ under a laser synthesis is a feasible process. While mixing the elemental powders in the specific stoichiometry and applying the threshold temperature specified in the phase diagram, the reaction for the formation of a silicide compound containing a high purity phase will probably occur. Thus, the formation of a $\mathrm{U}_{3} \mathrm{Si}_{2}$ phase may be feasible and will be confirmed with future experimentation.

\section{Conclusion}

The characterization of $\mathrm{U}_{3} \mathrm{Si}_{2}$ samples fabricated under a powder metallurgical approach confirmed the composition of the $\mathrm{U}_{3} \mathrm{Si}_{2}$ matrix along with the different secondary phases. Back scatter electron microscopy identified the phases present for quantification by means of digital image analysis. The porosity concentration averaged $0.91 \% \pm 0.47$ providing a low proportion which is desirable to preserve high densities. Electron microscopy (SEM and TEM) evaluated the samples' morphology, and the chemical analyses identified secondary phases on the fuel, including: $\mathrm{USi}, \mathrm{U}_{3} \mathrm{Si}$, and $\mathrm{UO}_{2}$. To validate the chemical analyses, the samples were analyzed by means of XRD. The diffraction patterns acquired confirmed the composition of the $\mathrm{U}_{3} \mathrm{Si}_{2}$ matrix, along with the presence of the following secondary phases: $\mathrm{USi}_{\mathrm{U}} \mathrm{UO}_{2}, \mathrm{U}_{3} \mathrm{Si}$, and metallic uranium.

The evaluation of higher $(7.5 \mathrm{wt} \%)$ silicon content on $\mathrm{U}_{3} \mathrm{Si}_{2}$ samples proved to increase the formation of the USi phase in the higher content samples when compared with the conventional (7.3 wt\%) silicon content. Additionally, the formation of $\mathrm{UO}_{2}$ was attributed to the presence of oxygen most probably while manufacturing the uranium and silicon powders. 
As proposed in Objective 3, the comparison between the AMAFT (surrogates) and the powder metallurgical $\left(\mathrm{U}_{3} \mathrm{Si}_{2}\right.$ fuel) samples' microstructure provided crucial data to formulate predictions of future $\mathrm{U}_{3} \mathrm{Si}_{2}$ samples undergoing a laser synthesis. The aforementioned results have increased the understanding in the formation of surrogate fuel prior to employing uranium compounds as part of the development of the AMAFT process.

The successful formation of the surrogate compounds $\mathrm{Zr}_{3} \mathrm{Si}_{2}$ and $\mathrm{Hf}_{3} \mathrm{Si}_{2}$ by means of a laser synthesis, provides the base process for the formation of $\mathrm{U}_{3} \mathrm{Si}_{2}$. The enthalpy of formation of $\mathrm{U}_{3} \mathrm{Si}_{2}$ is the highest among the surrogate compounds, and its negative Gibbs free energy ($84.56 \mathrm{KJ} / \mathrm{mol}$ ) suggests the occurrence of a spontaneous reaction. When combining the stoichiometric proportions of the compound at the threshold temperature, as suggested by the USi binary phase diagram, the formation of $\mathrm{U}_{3} \mathrm{Si}_{2}$ can be a feasible process. Ultimately, future experimentation with uranium compounds beyond the scope of this dissertation will provide the results to prove the feasibility of the additive manufacturing process to fabricate $\mathrm{U}_{3} \mathrm{Si}_{2}$ fuel.

The research work presented in this dissertation aimed to investigate and comply with Objectives 1, 2 and 3 (Chapter 1). For Objective 1, a survey of thermodynamic and mechanical properties of surrogate compounds was completed and allowed to examine the suitability of the selected materials to undergo a laser synthesis. In addition, the analyses performed on binary phase diagrams were crucial to perform experimentation with specific temperatures and stoichiometric ratios to form the desired compounds. Objective 2 included the characterization of surrogate fuel synthesized with a laser beam. The characterization results provided novel data that proved the suitability of the material and aid to incorporate modifications to implement the AMAFT process. Objective 3 emphasized on the characterization of $\mathrm{U}_{3} \mathrm{Si}_{2}$ fuel fabricated with a powder metallurgical approach. The $\mathrm{U}_{3} \mathrm{Si}_{2}$ characterization results were compared with those of 
surrogate compounds produced by AMAFT. The comparison assisted to predict the $\mathrm{U}_{3} \mathrm{Si}_{2}$ microstructure undergoing a laser synthesis. All characterization results have increased the understanding of the formation of surrogate silicide fuel prior to employing uranium compounds. 
Table 6-1. EDS quantification analysis on $\mathrm{U}_{3} \mathrm{Si}_{2}$ phase.

\begin{tabular}{ccc}
\hline Spot & Uranium (At. \%) & Silicon (At. \%) \\
\hline 1 & 64.26 & 35.74 \\
2 & 56.77 & 43.22 \\
3 & 55.07 & 44.92 \\
4 & 64.35 & 35.65 \\
5 & 70.77 & 29.23 \\
6 & 57.13 & 42.87 \\
7 & 58 & 42 \\
8 & 55.08 & 44.92 \\
9 & 66.03 & 33.97 \\
10 & 70.33 & 29.67 \\
\hline
\end{tabular}

Table 6-2. EDS quantification analysis on USi phase.

\begin{tabular}{ccc}
\hline Spot & Uranium (At. \%) & Silicon (At. \%) \\
\hline 1 & 48.69 & 51.31 \\
2 & 48.87 & 51.13 \\
3 & 49.31 & 50.69 \\
4 & 48.49 & 51.51 \\
5 & 50.58 & 49.42 \\
6 & 51.45 & 48.55 \\
7 & 49.12 & 50.88 \\
8 & 51.11 & 48.89 \\
9 & 51.91 & 48.09 \\
10 & 51.44 & 48.56 \\
11 & 50.73 & 49.27 \\
\hline
\end{tabular}

Table 6-3. EDS quantification analysis on $\mathrm{U}_{3} \mathrm{Si}$ phase.

\begin{tabular}{ccc}
\hline Spot & Uranium (At. \%) & Silicon (At. \%) \\
\hline 1 & 73.75 & 26.25 \\
2 & 73.99 & 26.01 \\
3 & 73.43 & 26.57 \\
4 & 73.75 & 26.25 \\
5 & 73.75 & 26.25 \\
6 & 73.99 & 26.01 \\
7 & 77.83 & 22.17 \\
8 & 73.43 & 26.57 \\
\hline
\end{tabular}


Table 6-4. EDS quantification analysis on TEM generated on $\mathrm{U}_{3} \mathrm{Si}_{2}$ samples

\begin{tabular}{ccccc}
\hline Sample & Spot & Uranium (At. \%) & Silicon (At. \%) & Oxygen (At. \%) \\
\hline A2 & 1 & 30.17 & 44.37 & 25.44 \\
A2 & 2 & 48.62 & 51.37 & 0 \\
A2 & 3 & 49.22 & 35.07 & 15.69 \\
A2 & 4 & 65.83 & 33.49 & 0.67 \\
D4 & 5 & 65.68 & 34.31 & 0 \\
D4 & 6 & 40.28 & 1.64 & 58.06 \\
C1 & 7 & 29.76 & 1.38 & 68.85 \\
C2 & 8 & 55.38 & 0 & 44.61 \\
\hline
\end{tabular}

Table 6-5. Key manufacturing parameters for $\mathrm{U}_{3} \mathrm{Si}_{2}$ pellets used in this study.

\begin{tabular}{ccccc}
\hline Sample & Wt\% Silicon & $\begin{array}{c}\text { Peak Sintering } \\
\text { Temperature }\left({ }^{\circ} \mathrm{C}\right)\end{array}$ & Sintering Atmosphere & $\begin{array}{c}\text { Measured } \\
\text { Density }\left(\mathrm{g} / \mathrm{cm}^{3}\right)\end{array}$ \\
\hline A2 & 7.5 & 1,550 & Argon & $11.54+/-0.06^{*}$ \\
D4 & 7.5 & 1,550 & Argon & $\begin{array}{c}\text { Density not } \\
\text { measured. }\end{array}$ \\
C & 7.3 & 1,550 & Vacuum & $11.8 * *$ \\
\hline
\end{tabular}

* Based on batch average.

** Based on batch average reported in (Harp, Lessing, \& Hoggan, 2015).

Table 6-6. Phase quantification results from digital image analysis.

\begin{tabular}{cccccc}
\hline Sample & $\begin{array}{c}\text { Silicon Content } \\
(\mathrm{Wt} \%)\end{array}$ & Porosity (\%) & $\mathrm{USi}(\%)$ & $\mathrm{UO}_{2}(\%)$ & $\begin{array}{c}\mathrm{U}_{3} \mathrm{Si}_{2} \mathrm{Matrix} \\
(\%)\end{array}$ \\
\hline $\mathrm{A}$ & 7.5 & $0.59 \pm 0.28$ & $13.35 \pm 4.39$ & $4.20 \pm 0.88$ & $81.86 \pm 4.89$ \\
$\mathrm{D}$ & 7.5 & $1.17 \pm 0.24$ & $9.85 \pm 1.87$ & $7.98 \pm 1.38$ & $81 \pm 2.55$ \\
$\mathrm{C}$ & 7.3 & $0.80 \pm 0.47$ & $7.65 \pm 2.92$ & $5.57 \pm 0.70$ & $85.98 \pm 2.81$ \\
\hline
\end{tabular}



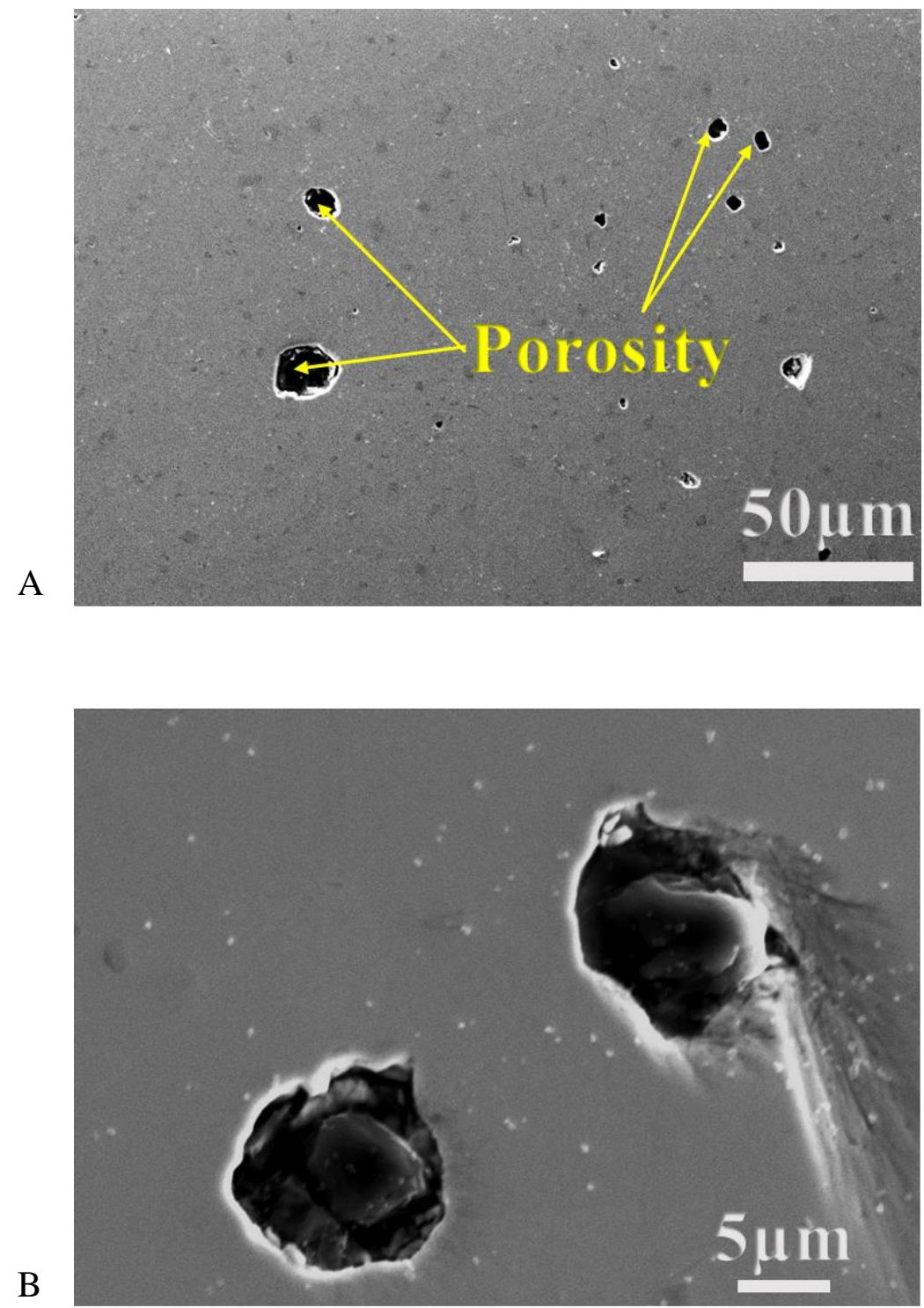

Figure 6-1. SEM images displaying the microstructure of $\mathrm{U}_{3} \mathrm{Si}_{2}$ fabricated by means of a powder metallurgical process. A) SEM micrograph displaying porosity found on $\mathrm{U}_{3} \mathrm{Si}_{2}$ sample. B) SEM micrograph magnified to $2,700 \times$ displaying porosity. 


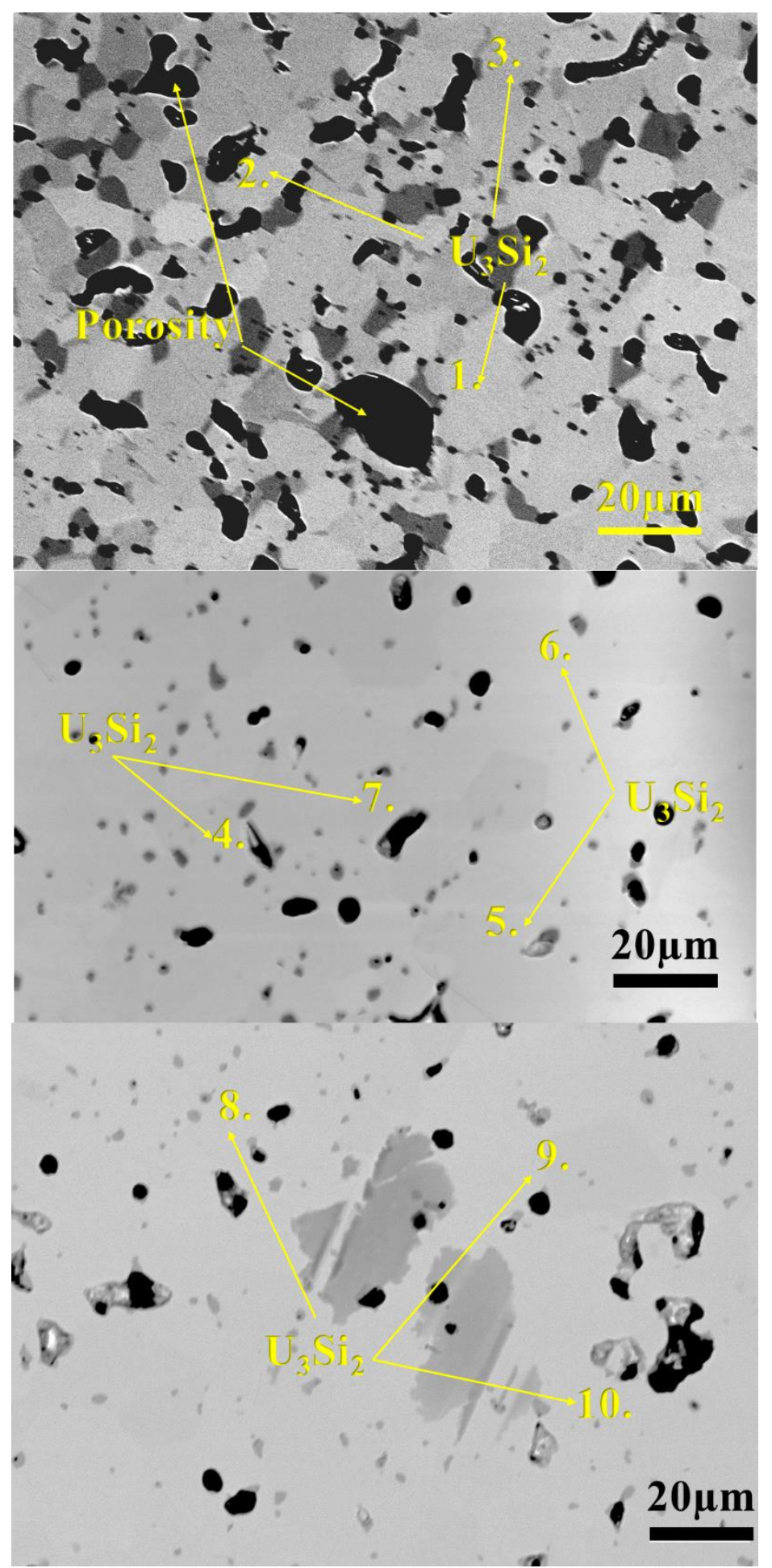

Figure 6-2. Electron backscatter micrographs displaying areas with high concentration of $\mathrm{U}_{3} \mathrm{Si}_{2}$ confirmed by EDS. 


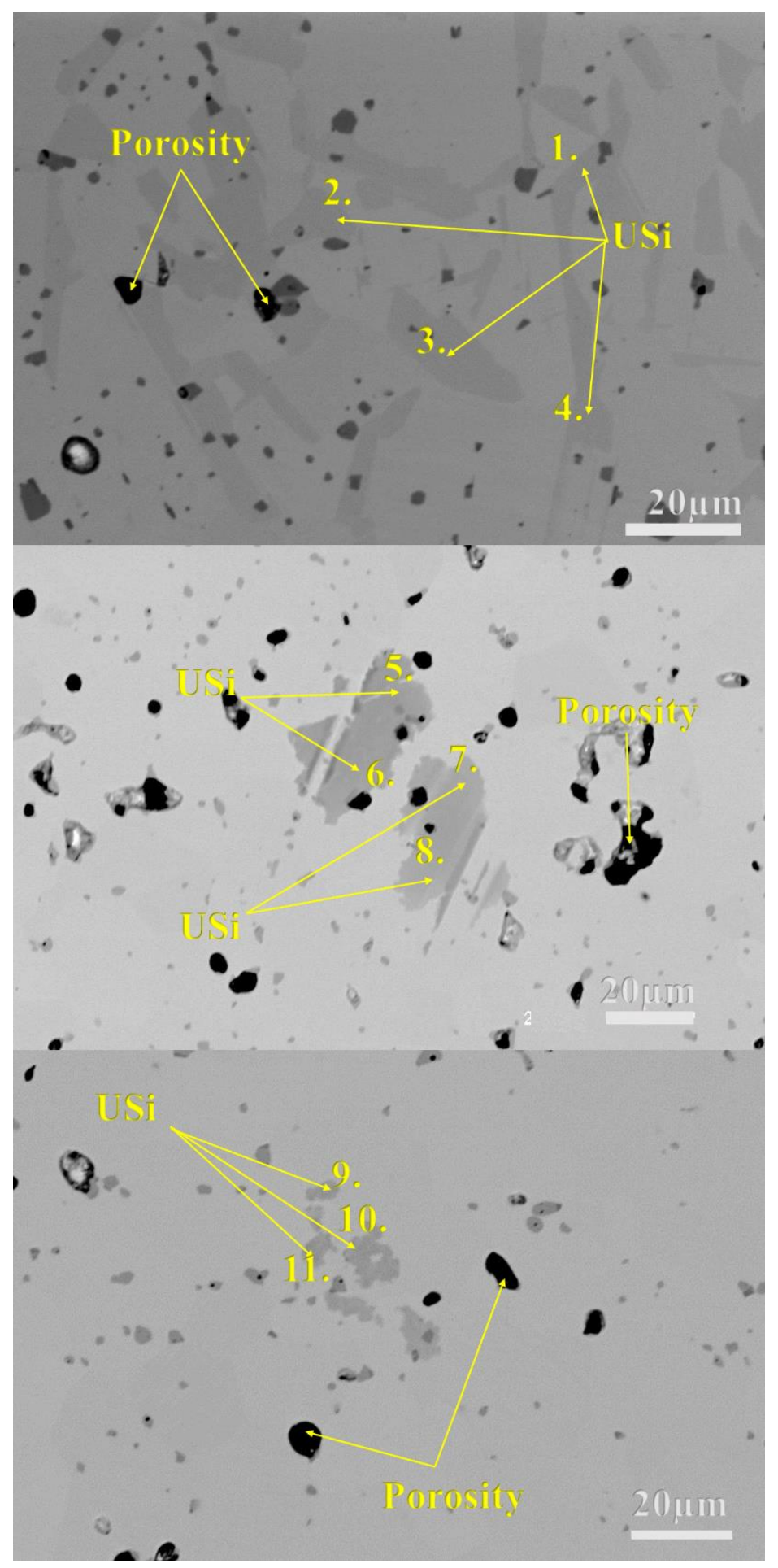

Figure 6-3. Electron backscatter micrographs displaying areas with high concentration of USi confirmed by EDS. 


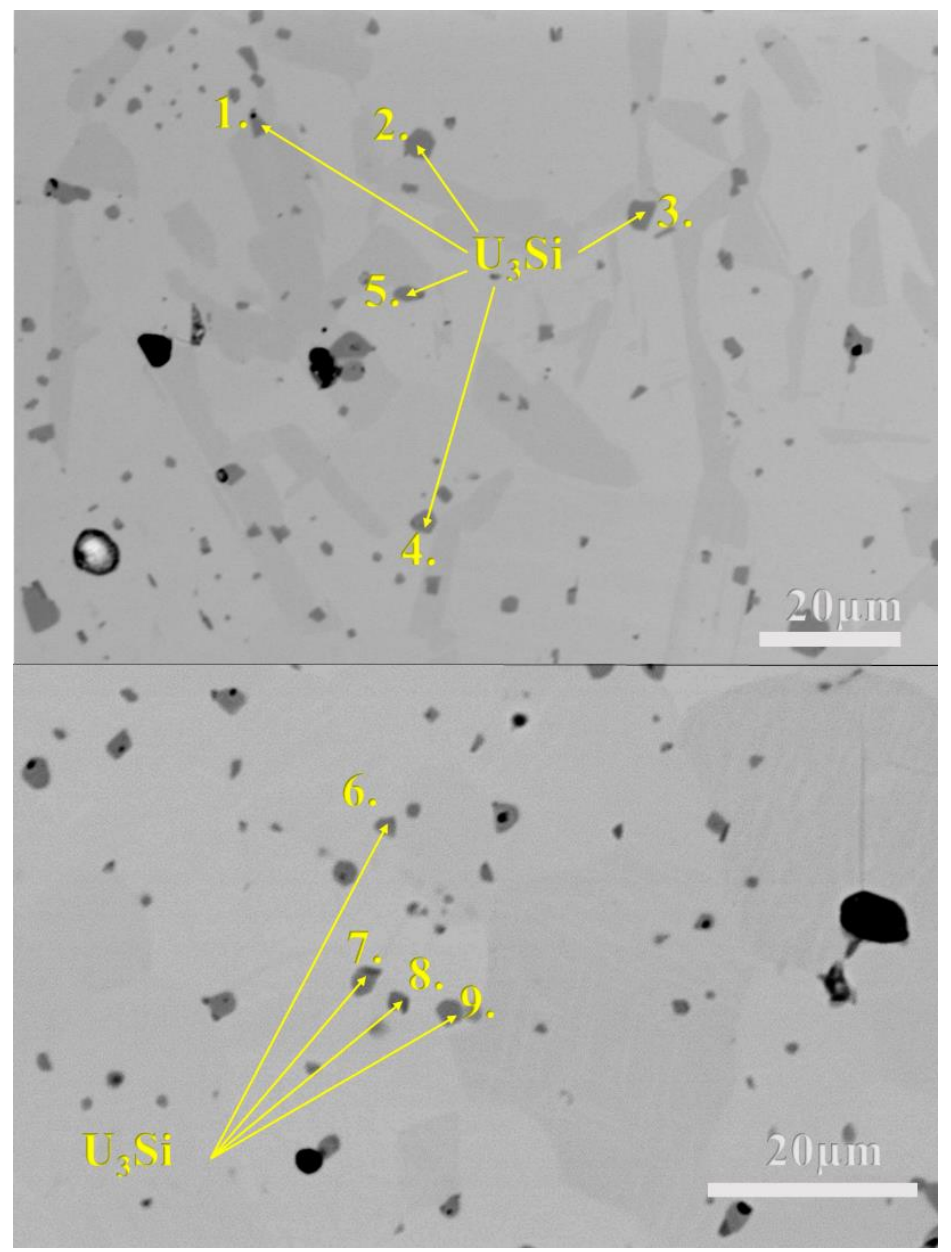

Figure 6-4. Electron backscatter micrographs displaying areas with high concentration of $\mathrm{U}_{3} \mathrm{Si}$ confirmed by EDS. 

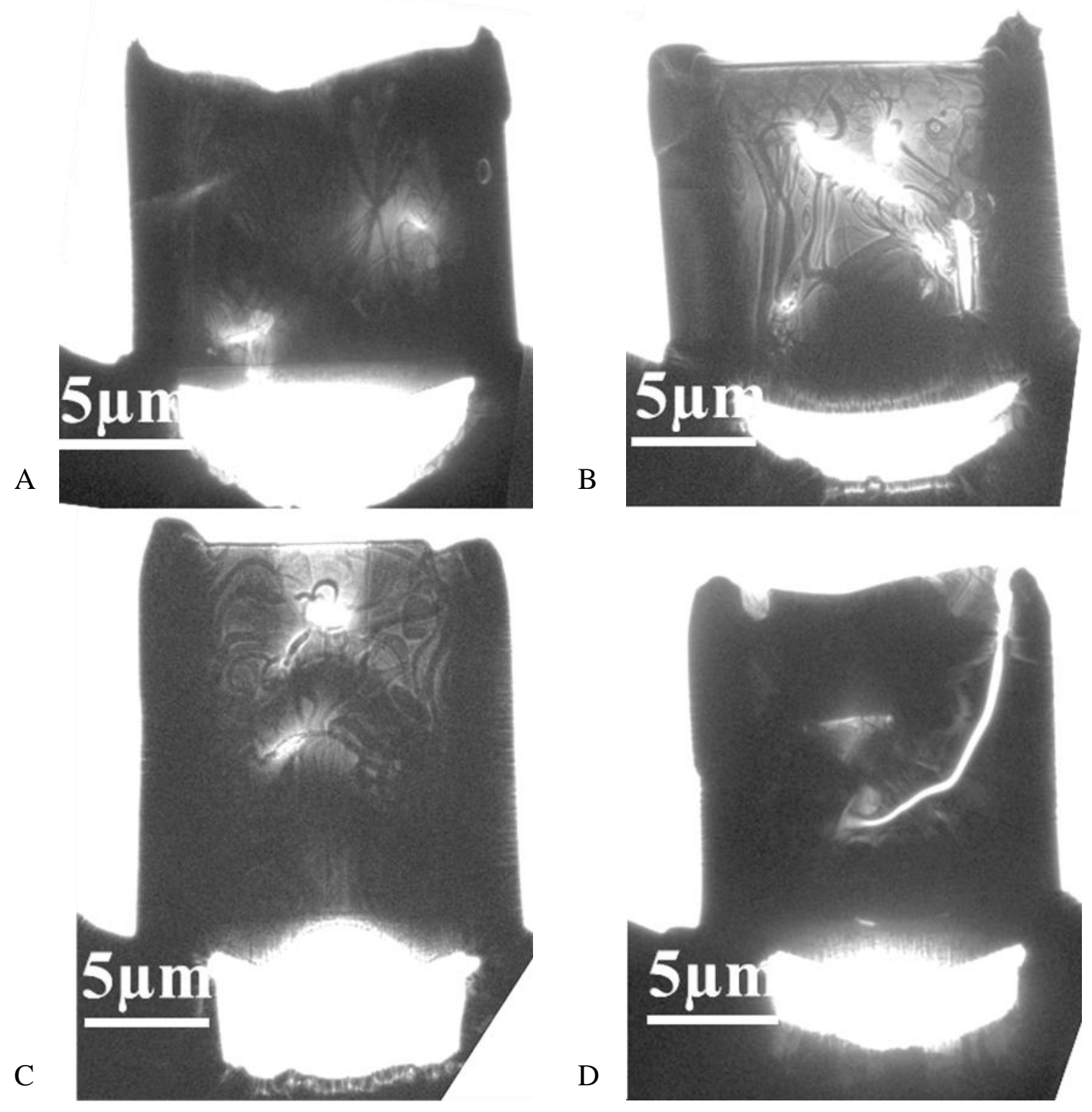

Figure 6-5. TEM lamellae. A) Sample A2. B) Sample D4. C) Sample C1. D) Sample C2. 

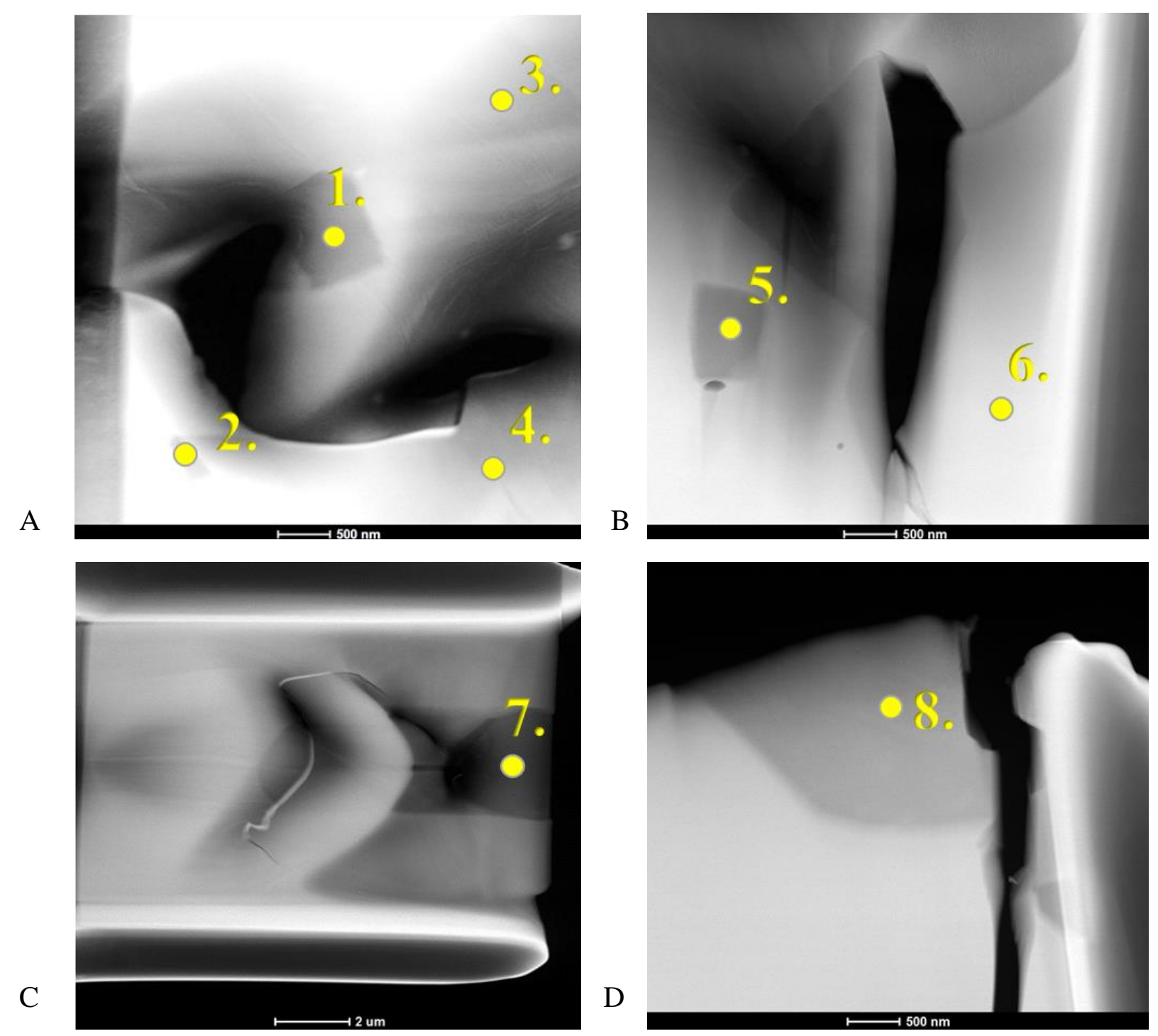

Figure 6-6. TEM micrographs where chemical analyses were performed on $\mathrm{U}_{3} \mathrm{Si}_{2}$ samples. A) Sample A2. B) Sample D4. C) Sample C1. D) Sample C2. 

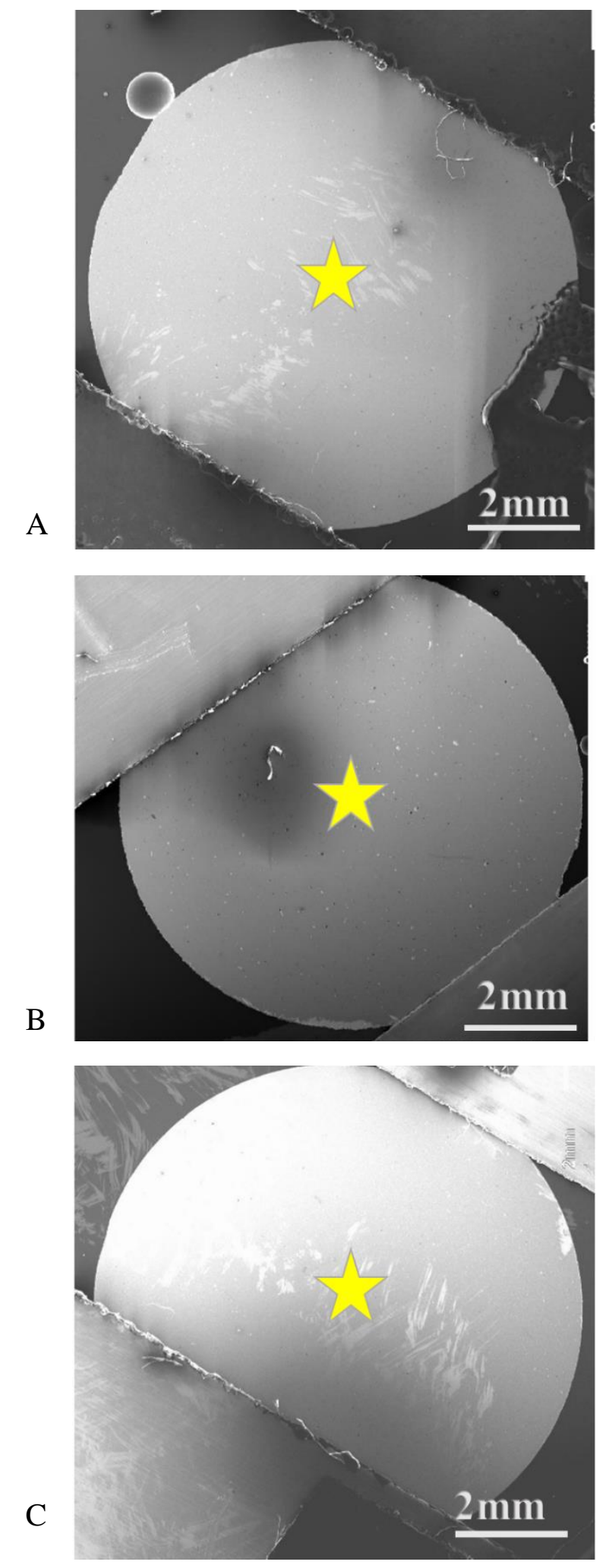

Figure 6-7. SEM micrographs of $\mathrm{U}_{3} \mathrm{Si}_{2}$ samples where XRD analyses where performed. A) Sample A2. B) Sample D4. C) Sample C. 


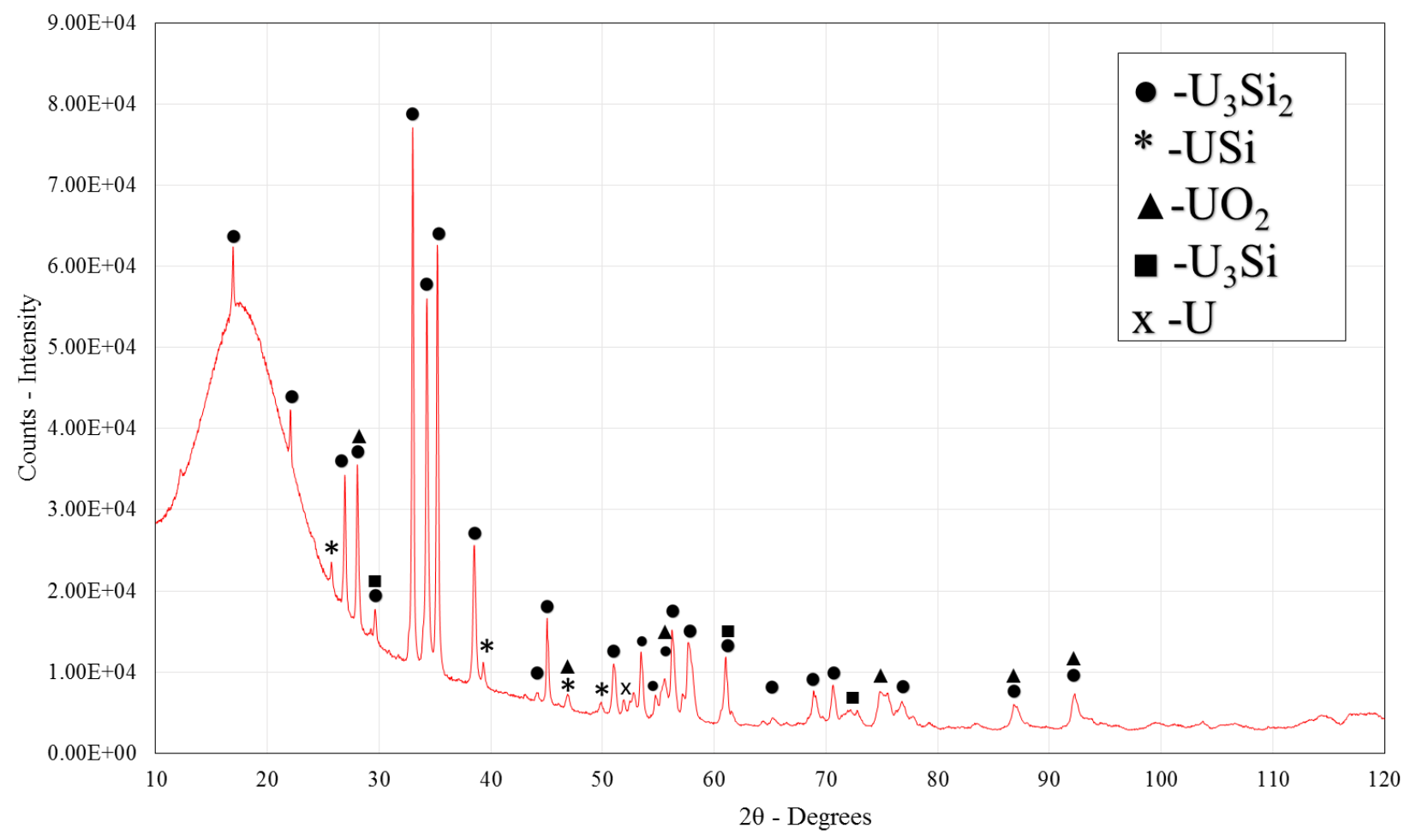

Figure 6-8. XRD analysis of $\mathrm{U}_{3} \mathrm{Si}_{2}$ Sample A2.

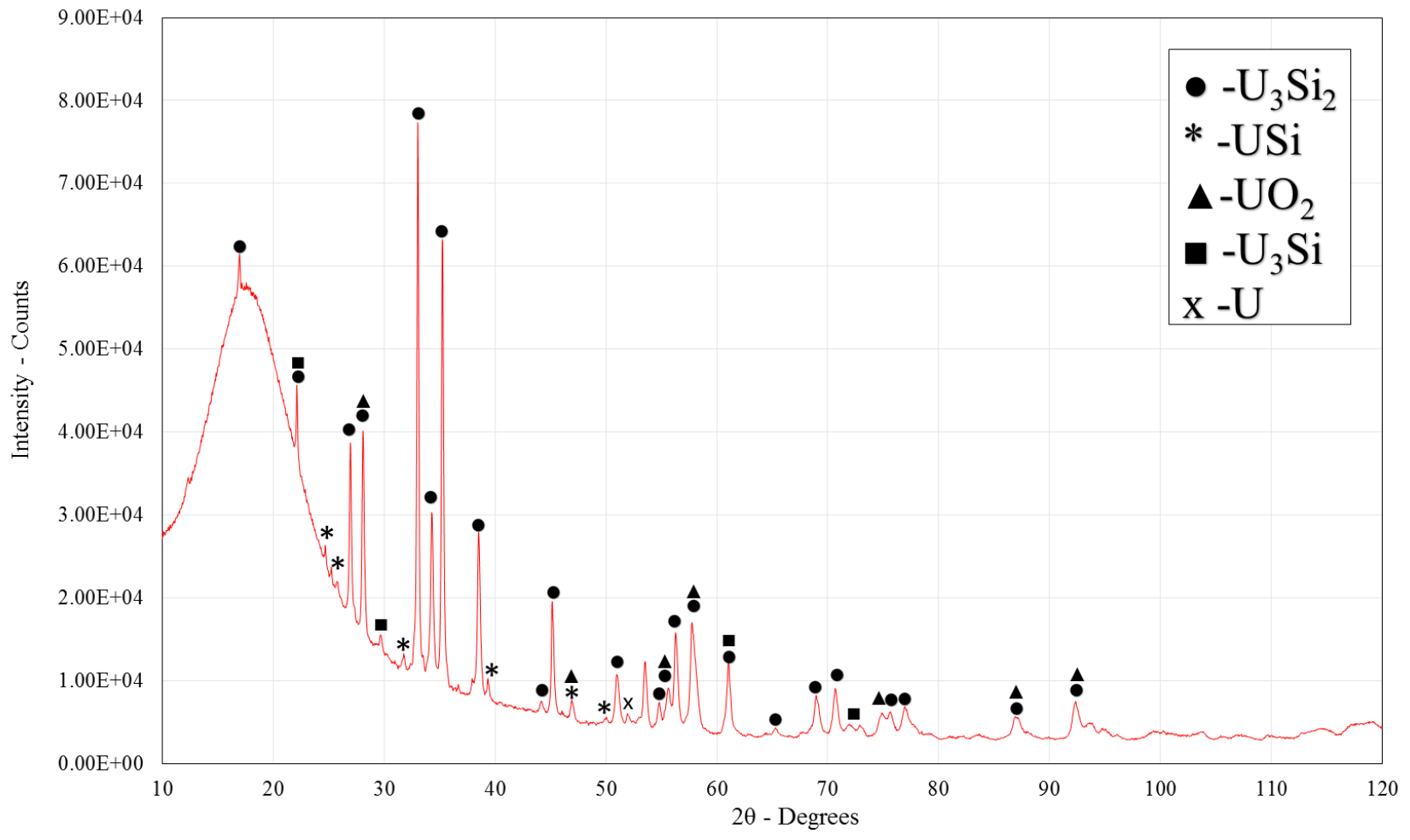

Figure 6-9. XRD analysis of $\mathrm{U}_{3} \mathrm{Si}_{2}$ Sample D4. 


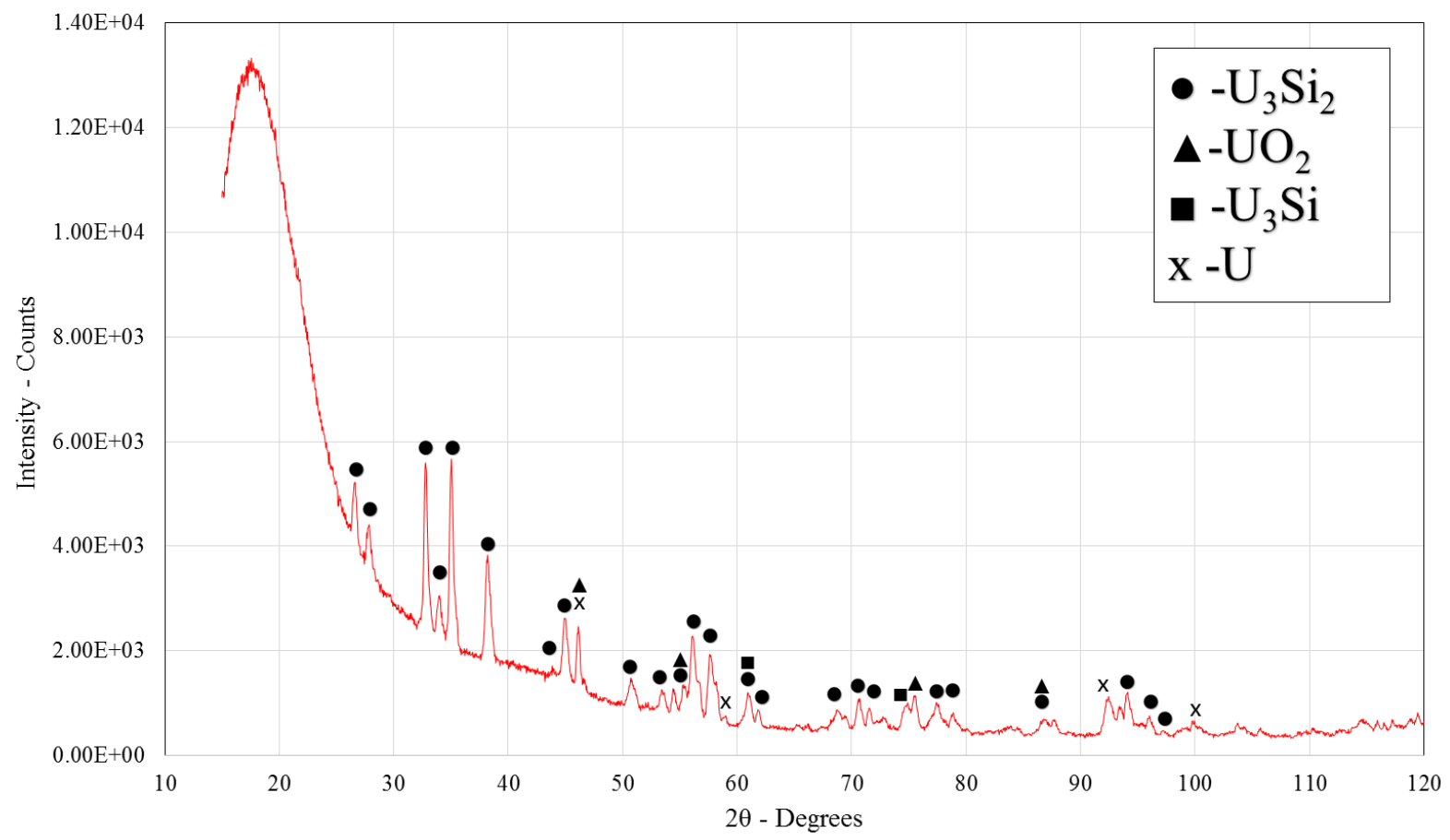

Figure 6-10. XRD analysis of $\mathrm{U}_{3} \mathrm{Si}_{2}$ Sample C.

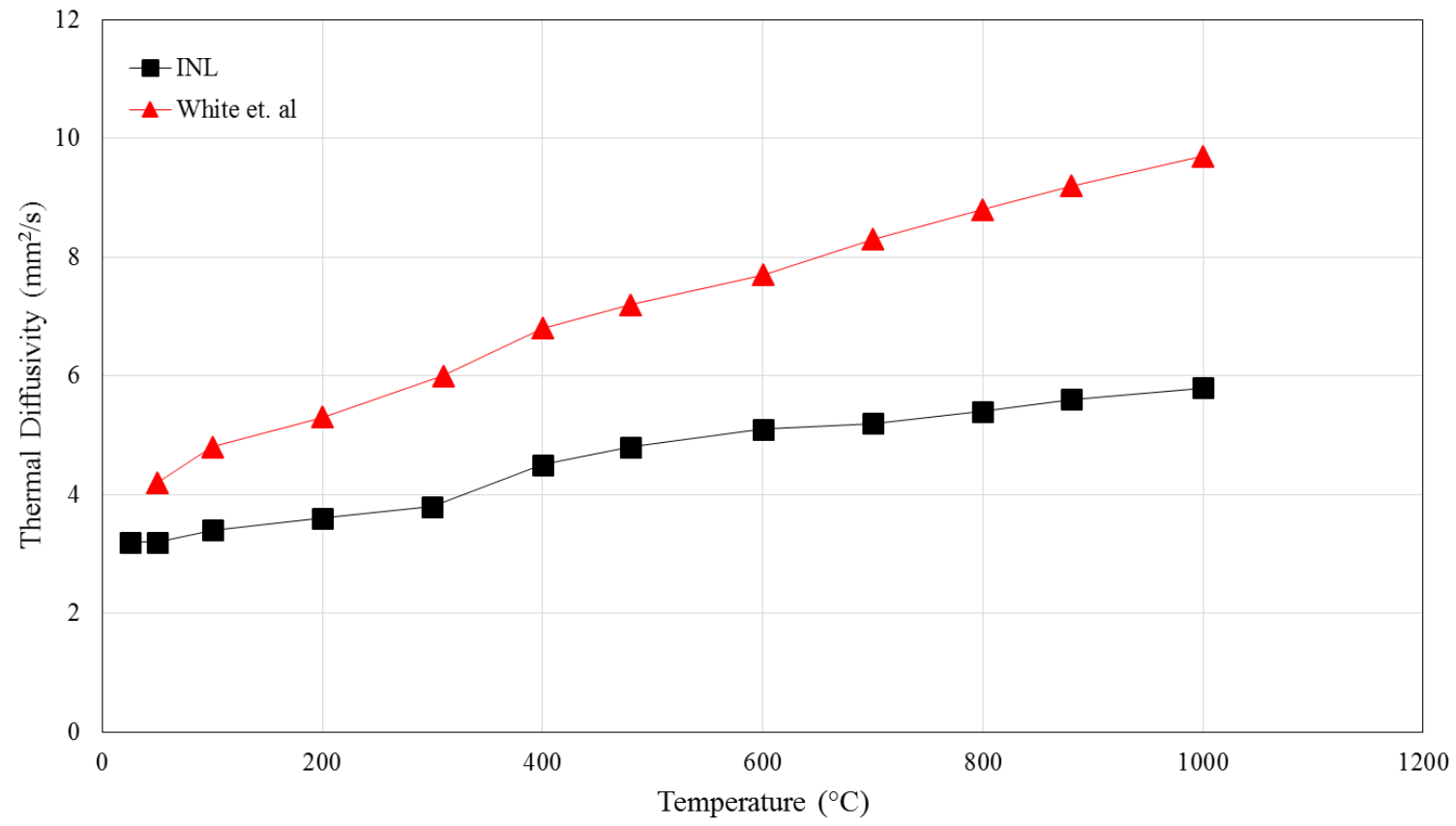

Figure 6-11. Thermal diffusivity of $\mathrm{U}_{3} \mathrm{Si}_{2}$ samples as a function of temperature. The collected measurements are being compared with the work by White [16]. 

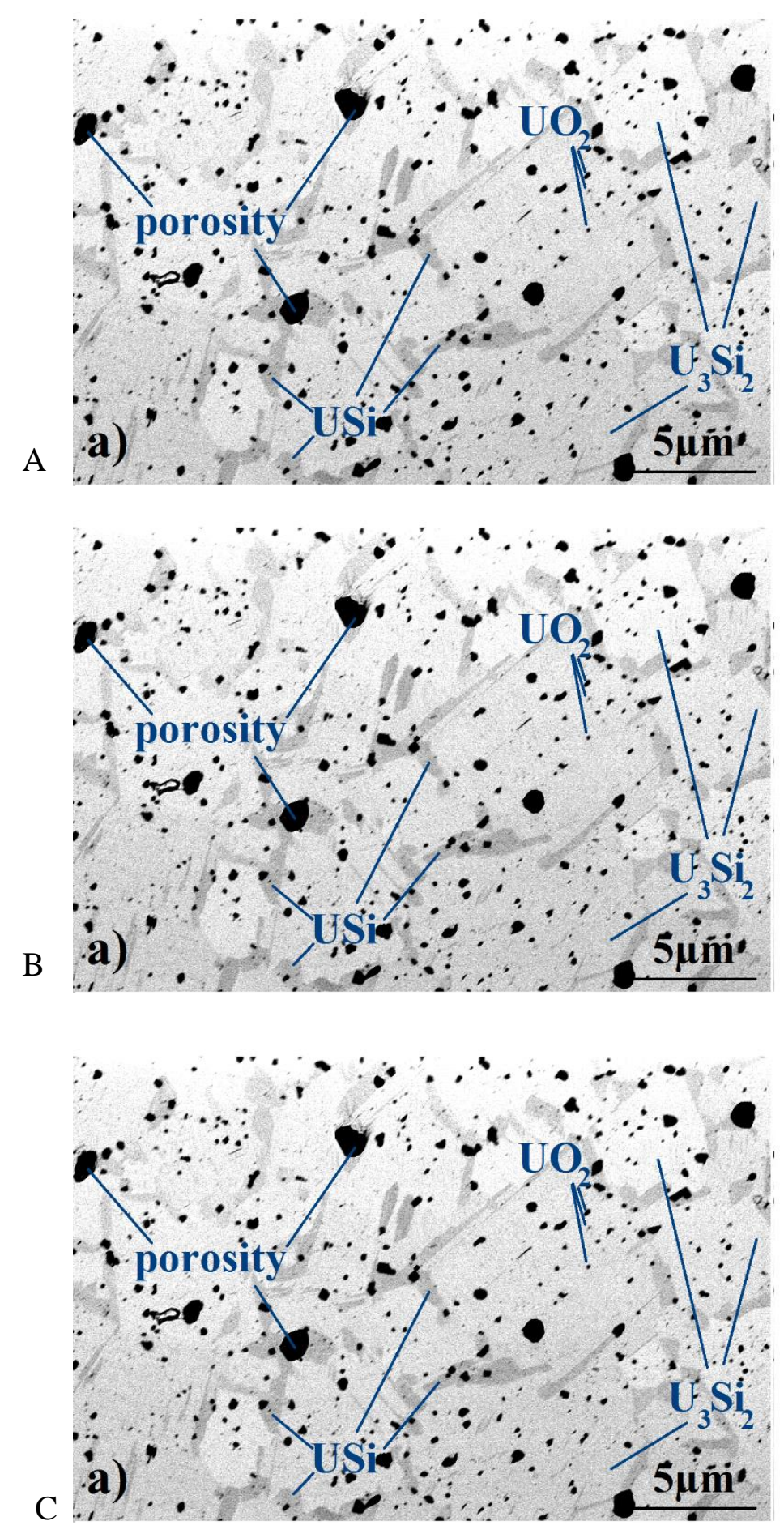

Figure 6-12. Back scatter SEM images of $\mathrm{U}_{3} \mathrm{Si}_{2}$ samples. A) Sample A2 with7.5 wt.\% Si. B) Sample D4 with 7.5 wt.\% Si. C) Sample C with 7.3 wt\% Si [43]. 


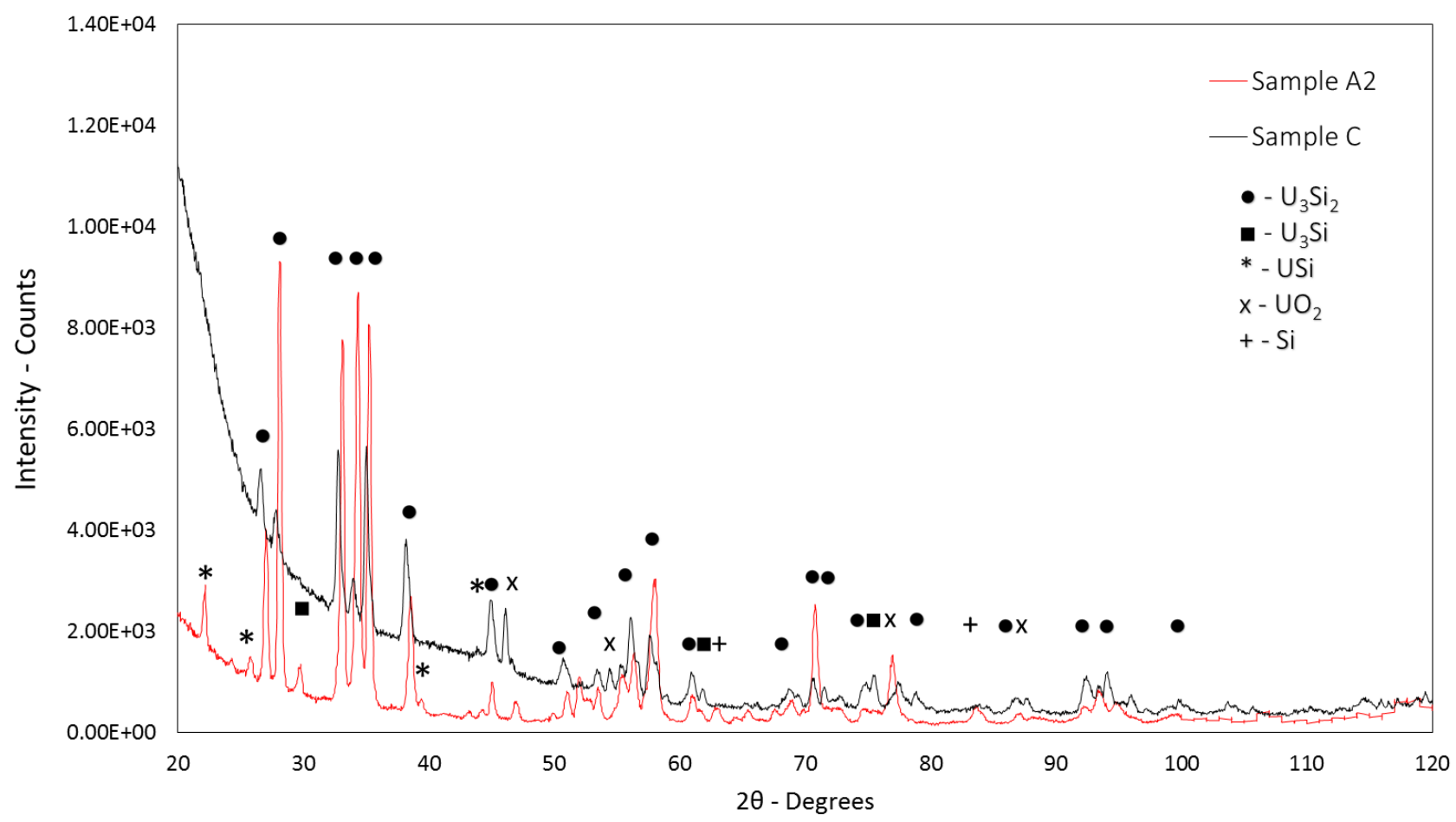

Figure 6-13. XRD analysis of Samples A2 and C.

A

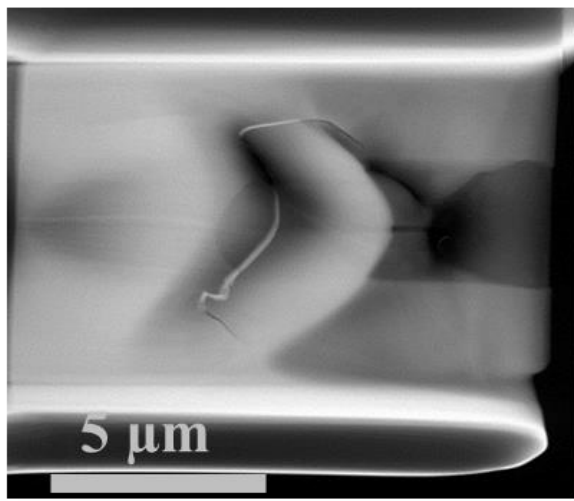

$\mathrm{C}$

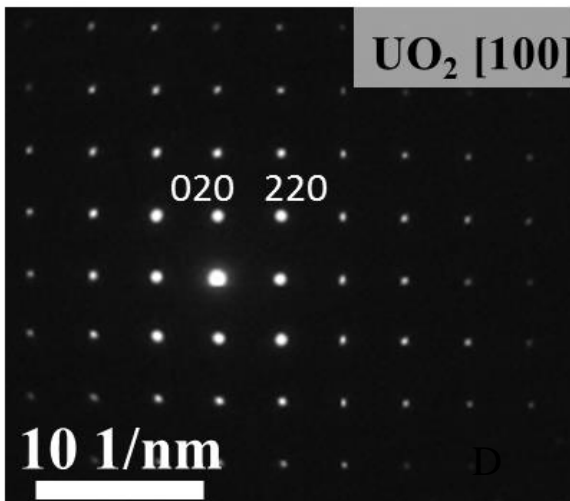

B

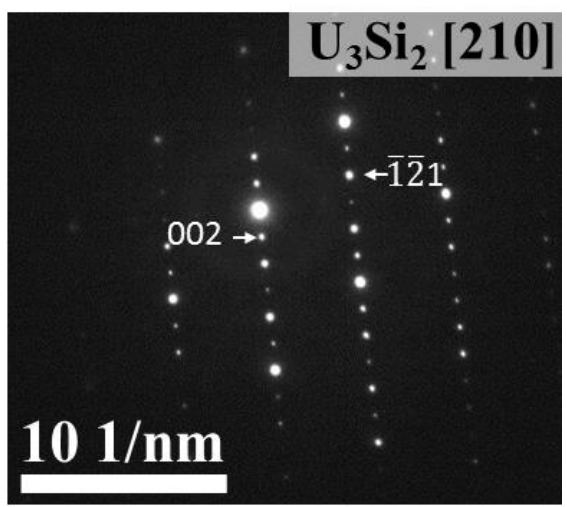

$\mathrm{D}$

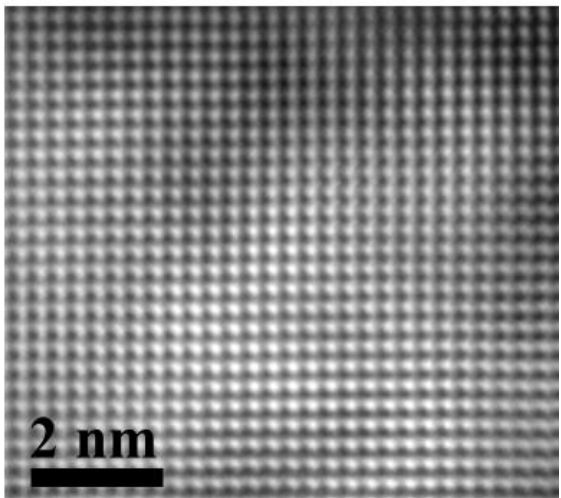

Figure 6-14. Images displaying $\mathrm{SAD}$ results on $\mathrm{U}_{3} \mathrm{Si}_{2}$ samples. A) STEM image of Sample C (7.3 wt\% Si). B) SAD pattern of the matrix. C) SAD pattern on grain displayed on Figure 6-14 (A). D) HRTEM image of the $\mathrm{UO}_{2}$ grain. 

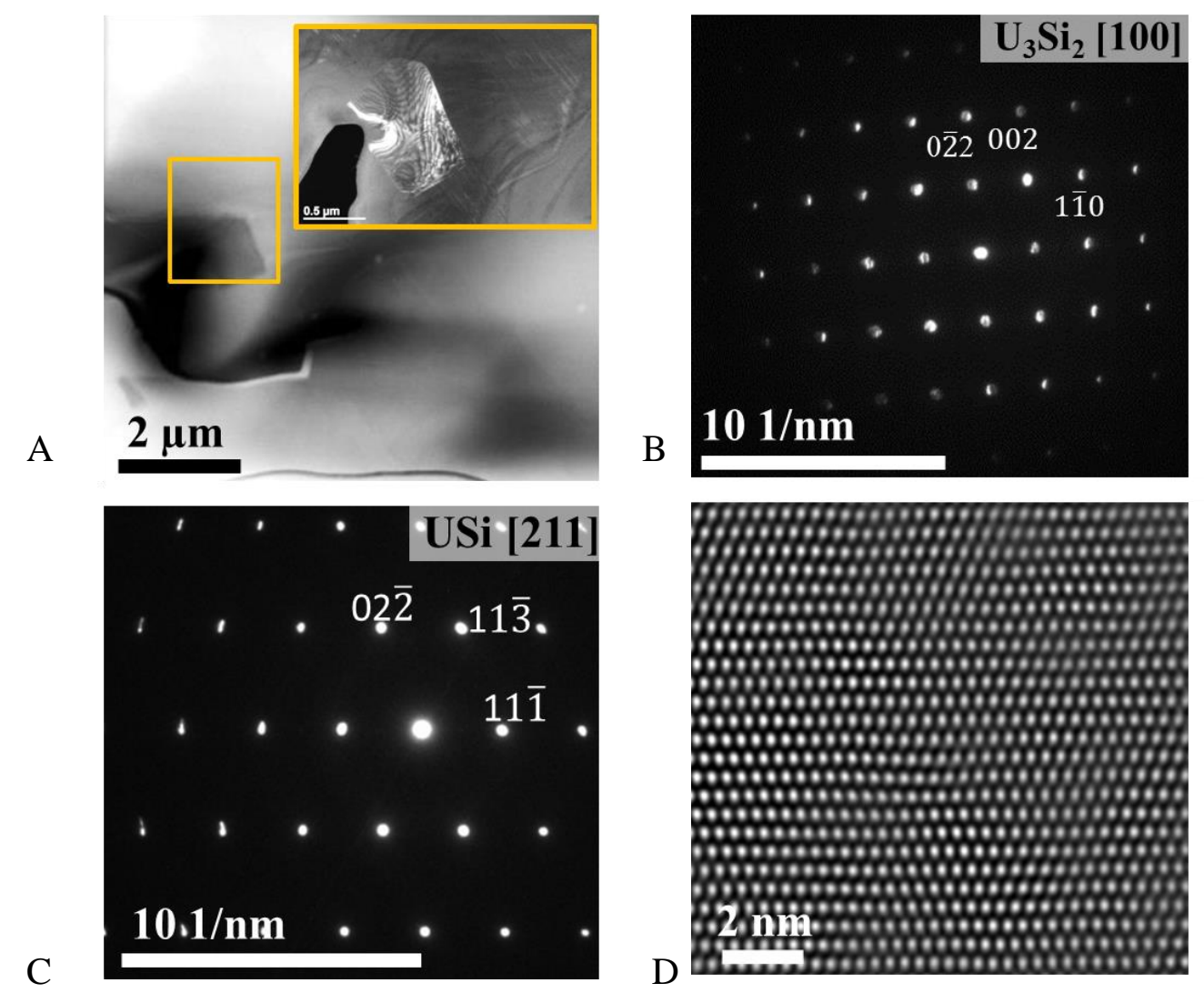

Figure 6-15. Images displaying SAD results on $\mathrm{U}_{3} \mathrm{Si}_{2}$ samples. A) STEM image of Sample A2 (7.5 wt \% Si). B) SAD on the matrix of Sample A2 displaying a tetragonal crystal structure. C) SAD on grain displayed on Figure 6-15 (A). D) HRTEM image of the USi grain. 

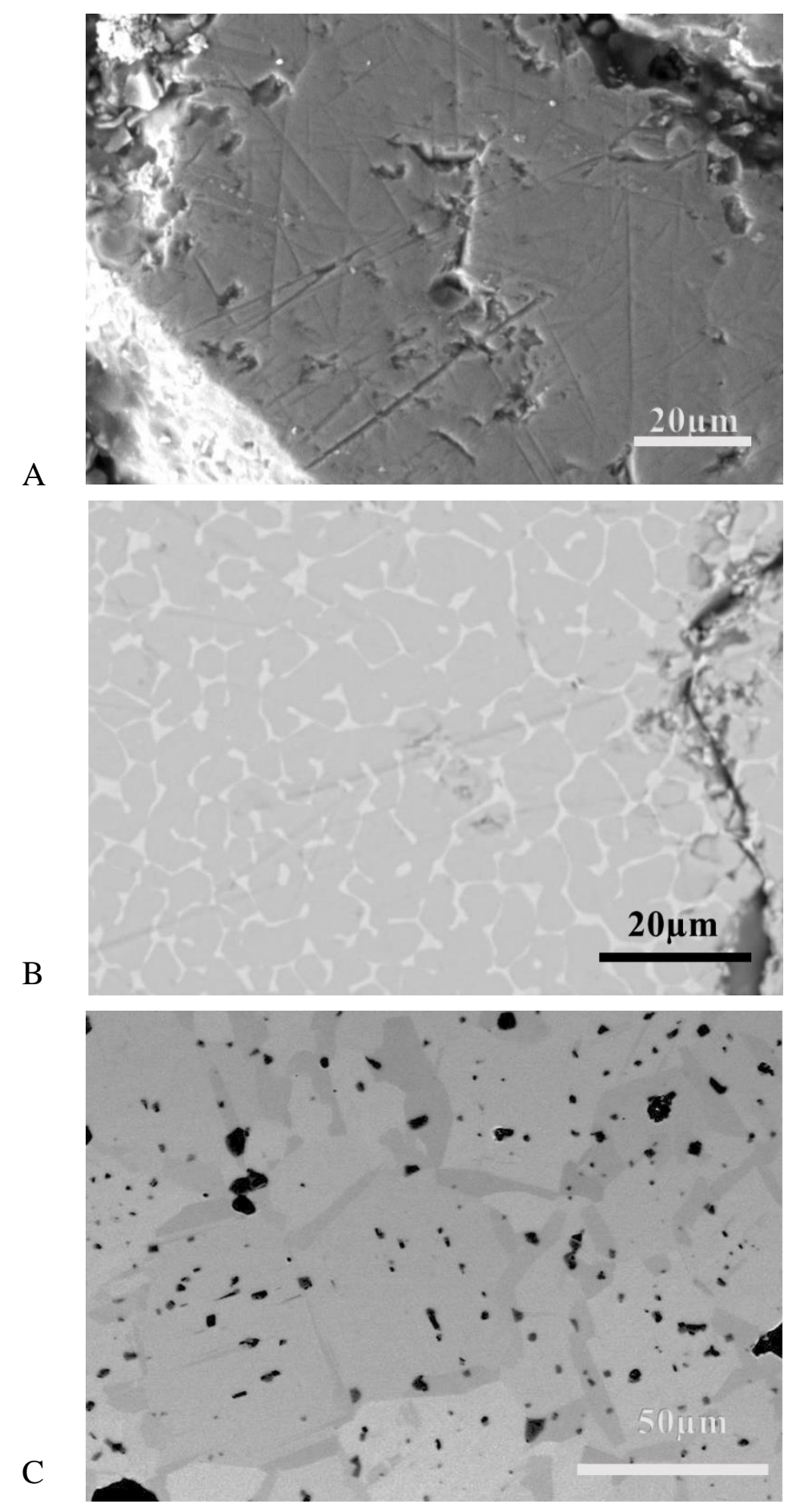

Figure 6-16. SEM images comparing the morphology of $\mathrm{U}_{3} \mathrm{Si}_{2}$ and $\mathrm{Zr}_{3} \mathrm{Si}_{2}$ compounds. A) SEM micrograph displaying the morphology of a laser synthesized $\mathrm{Zr}_{3} \mathrm{Si}_{2}$ compound. B) Back-scatter electron micrograph displaying grain formation of $\mathrm{Zr}_{3} \mathrm{Si}_{2}$. C) Backscatter electron micrograph displaying $\mathrm{U}_{3} \mathrm{Si}_{2}$ morphology and different phases present. 

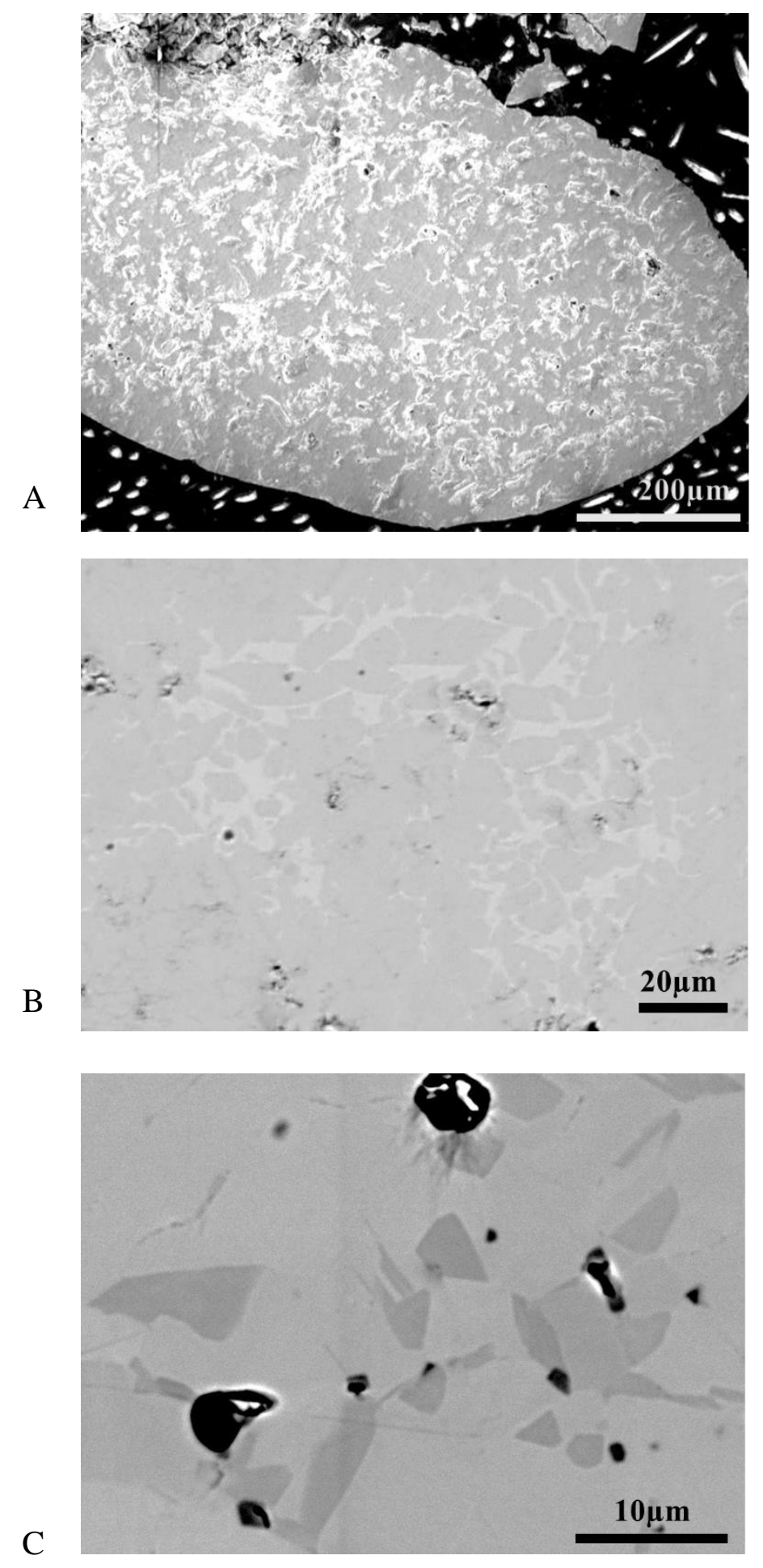

Figure 6-17. SEM micrographs comparing the morphology of $\mathrm{Hf}_{3} \mathrm{Si}_{2}$ and $\mathrm{U}_{3} \mathrm{Si}_{2}$ samples. A) SEM micrograph displaying the morphology of a laser synthesized $\mathrm{Hf}_{3} \mathrm{Si}_{2}$ sample. B) Back-scatter electron micrograph displaying phase formation of $\mathrm{Hf}_{3} \mathrm{Si}_{2}$. C) Backscatter electron micrograph displaying the morphology of $\mathrm{U}_{3} \mathrm{Si}_{2}$ along with different phases present. 


\section{CHAPTER 7 \\ CONCLUSIONS AND FUTURE WORK}

\section{Conclusions}

A thermodynamic study assisted in understanding the principle regulating the heat transformation in the selected surrogates. Thermodynamic and mechanical properties of the down selected compounds were investigated to prove the suitability of the materials to undergo laser synthesis. The down-selection of the surrogate materials was established based on the crystallographic structure of the compounds, all preserving a tetragonal crystal structure $\left(\mathrm{U}_{3} \mathrm{Si}_{2}\right.$, $\mathrm{Ce}_{3} \mathrm{Si}_{2}, \mathrm{Zr}_{3} \mathrm{Si}_{2}$, and $\mathrm{Hf}_{3} \mathrm{Si}_{2}$ ) along with similarities encountered on their respective binary phase diagrams. The most significant similarity to the U-Si binary system was the multiple eutectic points, which allow the formation of the desired compounds, along with different secondary phases on each binary system. The analyses performed on phase diagrams of surrogate materials were vital to define the exact proportions of the sample powders and threshold temperatures to form surrogate compounds with the desired stoichiometry. Additionally, the binary phase diagrams allowed prediction of secondary phases that can potentially appear due to an uneven heat distribution from the laser sintering source.

The synthesis of zirconium silicide successfully produced the desired compound, $\mathrm{Zr}_{3} \mathrm{Si}_{2}$, under this novel laser methodology. Also, the formation of surrogate silicide fuel, $\mathrm{Hf}_{3} \mathrm{Si}_{2}$, by means of a laser synthesis, proved to be a success. The chemical analyses of both $\mathrm{Zr}_{3} \mathrm{Si}_{2}$ and $\mathrm{Hf}_{3} \mathrm{Si}_{2}$, suggested the presence of secondary phases that were also confirmed by XRD. Though prior literature presented difficulties in forming silicide compounds with an exact stoichiometry and only a pure phase present, ongoing modifications to the laser parameters are being incorporated to ultimately minimize the formation of secondary phases. Nonetheless, the results 
from the surrogate compounds have provided microstructural data to design the experimental set up to produce uranium compounds by means of an additive manufacturing process.

Among the selected surrogates, $\mathrm{Zr}_{3} \mathrm{Si}_{2}$ was the most relevant for this work. Besides its successful trajectory as a uranium surrogate for research purposes, $\mathrm{Zr}_{3} \mathrm{Si}_{2}$ can also be incorporated in different applications within the nuclear industry (ie. Gen IV reactor reflector).

The characterization of $\mathrm{U}_{3} \mathrm{Si}_{2}$ samples fabricated under a powder metallurgical approach assisted in confirming the composition of the $\mathrm{U}_{3} \mathrm{Si}_{2}$ matrix along with the secondary phases from the U-Si binary system. A microstructural comparison between surrogates and $\mathrm{U}_{3} \mathrm{Si}_{2}$ revealed the presence of an inhomogeneous morphology along with secondary phases in both, surrogate and $\mathrm{U}_{3} \mathrm{Si}_{2}$ samples. All phases were confirmed by XRD. They belonged to the respective binary system (Hf-Si, Zr-Si, and U-Si).

The current research work has set the process parameters for future work with uranium compounds. Based on experimental results, the concept of forming surrogate silicide fuel $\left(\mathrm{Zr}_{3} \mathrm{Si}_{2}\right.$ and $\mathrm{Hf}_{3} \mathrm{Si}_{2}$ ) employing a laser energy source was validated. If performed under the same process parameters, the formation of a $\mathrm{U}_{3} \mathrm{Si}_{2}$ compound from uranium and silicon powders may be achievable. These unique characterization results from the surrogate compounds are available for future laser based experimental work and will provide a data base for future uranium surrogate choices. This work highly supported the development of the AMAFT process to fabricate accident tolerant fuel. Upon completion, the AMAFT process envisages to significantly shorten the fabrication process of $\mathrm{U}_{3} \mathrm{Si}_{2}$ fuel, which possess enhanced thermal properties, higher atomic density, and improved irradiation stability when compared to $\mathrm{UO}_{2}$. Ultimately, this accident tolerant fuel candidate will provide clean and reliable energy to benefit human kind. 


\section{Future Work}

This was the first additive manufacturing project ever funded by the U.S. Department of Energy. Groundbreaking milestones were completed while successfully attaining the desired surrogate phases. Further development can be implemented in order to optimize the process parameters and fully develop the AMAFT method. Additional work may include the experimentation with smaller particle size on the sample powders. A smaller particle size can potentially assist in developing an improved surface finish during the final stages of the AMAFT process. The formation of the desired phases $\mathrm{Zr}_{3} \mathrm{Si}_{2}$ and $\mathrm{Hf}_{3} \mathrm{Si}_{2}$ was successfully accomplished. In order to enhance the purity of the compounds $\left(\mathrm{Zr}_{3} \mathrm{Si}_{2}\right.$ and $\left.\mathrm{Hf}_{3} \mathrm{Si}_{2}\right)$, a metallic hyper and hypostoichiometric ratio on the sample powders can be tested during the synthesis in order to evaluate the possibility of increasing the phase quantification fraction $\left(\mathrm{Zr}_{3} \mathrm{Si}_{2}\right.$ and $\left.\mathrm{Hf}_{3} \mathrm{Si}_{2}\right)$ upon solidification of the sample. The laser material interaction should be studied further to develop optimum parameters to produce surrogate silicide fuel. Thus, a thorough study on the effect of various power outputs (W) at different pulse-widths (ms) can be generated on the sample materials to understand the heat dissipation, void formation, and complete sintering of the powders. These are only a few areas that will require further study, nonetheless, as the need for further development comes, other areas will be researched to fully develop the AMAFT process. 


\section{LIST OF REFERENCES}

[1] T. WIENCEK, "Summary report on fuel development and miniplate fabrication for the RERTR program, 1978 to 1990,” ANL/RERTR/TM-15, Argonne National Laboratory, (1995).

[2] CARLETON COLLEGE, The Science Education Resource Center, Scanning Electron Microscopy (SEM), https://serc.carleton.edu/research_education/geochemsheets/techniques/SEM.html., Accessed (Dec. 2017).

[3] NANOSCIENCE INSTRUMENTS, How an SEM Works, http://www.nanoscience.com/technology/sem-technology/how-sem-works/, Accessed (Dec. 2017).

[4] UNIVERSITY OF WARWICK, Department of Physics, Transmission Electron Microscopy (TEM), https://warwick.ac.uk/fac/sci/physics/current/postgraduate/regs/mpagswarwick/ex5/techniq ues/structural/tem/, Accessed (Dec. 2017).

[5] S. WISCHNITZER, Introduction to Electron Microscopy, Pergamon Press, New York (1980).

[6] G. ALANKO, B. JACQUES, ALLYSSA BATEMAN, and DARRYL BUTT, "Mechanochemical synthesis and spark plasma sintering of the cerium silicides," Journal of Alloys and Compounds, 616, 306 (2014).

[7] P. EDMONSON, Y. ZHANG, S. MOLL, F. NAMAVAR, and W. WEBER, "Irradiation effects on microstructure change in nanocrystalline ceria-Phase, lattice stress, grain size and boundaries," Acta Materialia, 60, 5408 (2012).

[8] A. KERCHER and J. D. HUNN, "Results from ORNL characterization of $\mathrm{ZrO}_{2}-500-\mathrm{AK} 2-$ surrogate TRISO material,” ORNL/TM-2005/540, Oak Ridge National Laboratory, (2005).

[9] F. GUITTONNEAU, A. ABDELOUAS, B. GRAMBOW, M. DIALINAS, and F. CELLIER, "New Methods for HTR Fuel Waste Management," Fourth International Topical Meeting on High Temperature Reactor Technology, American Society of Mechanical Engineers, (2008).

[10] J. HAN and Y. LI, "Interaction Between Pulsed Laser and Materials," Laser Applications in Science and Industry, InTech (2011).

[11] M. JAN, "Chapter 1: Review of laser matter-interaction," Optical Institute of Spain IOCSIC, Madrid, (2011). 
[12] V. KEMLIN, "Second Harmonic Generation" (digital image), Videos on Second Harmonic Generation, Web, Accessed (Mar. 2017).

[13] V. VIKHRENKO, Heat Transfer - Engineering Applications, Chap. 3, InTech, Croatia (2011).

[14] J. HARP, P. LESSING, and R. HOGGAN, "Uranium silicide pellet fabrication by powder metallurgy for accident tolerant fuel evaluation and irradiation," Journal of Nuclear Materials, 466, 728 (2015).

[15] H. FOXHALL, and D. GODDARD, “Thermodynamic Modelling of a Single Stage Production Route for $\mathrm{U}_{3} \mathrm{Si}_{2}$ Accident Tolerant Fuel," Top Fuel Conference, Zurich, Switzerland, September 13-17, 2015, p. 166 (2015).

[16] J. WHITE, A. NELSON, J. DUNWOODY, D. BYLER, D. SAFARIK, and K. MCCLELLAN, "Thermophysical properties of $\mathrm{U}_{3} \mathrm{Si}_{2}$ to $1,773 \mathrm{~K}$." Journal of Nuclear Materials, 464, 275 (2015).

[17] V. SINHA, G. MISHRA, S. PAL, K. KHAN, P. HEGDE, and G. PRASAD, "Development of powder metallurgy technique for synthesis of $\mathrm{U}_{3} \mathrm{Si}_{2}$ dispersoid," Journal of Nuclear Materials, 383, 196 (2008).

[18] G. ALANKO and D. BUTT, "Mechanochemical synthesis of uranium sesquisilicide," Journal of Nuclear Materials, 451, 243 (2014).

[19] K. KIM, D. LEE, C. KIM, H. IL, and K. PAIK, "Characteristics of $\mathrm{U}_{3} \mathrm{Si}$ and $\mathrm{U}_{3} \mathrm{Si}_{2}$ powders prepared by centrifugal atomization," Journal of Nuclear Science and Technology, 34, 1127 (1997).

[20] K. KIM, "Thermal compatibility studies of $\mathrm{U}_{3} \mathrm{Si}_{2}$ dispersion fuels prepared with centrifugally atomized powder," Journal of Nuclear Materials, 270, 315 (1999).

[21] C. KIM, C. LEE, H. PARK, K. KIM, and I. KUK, The effect of particle shape on thermal and electrical conductivities of uranium silicide dispersion fuel, JAERI-M--94-042, Japan Atomic Energy Research Institute (1994).

[22] X. WU, "Preliminary safety analysis of the PWR with accident-tolerant fuels during severe accident conditions," Annals of Nuclear Energy, 80, 10 (2015).

[23] C. AZEVEDO, "Selection of fuel cladding material for nuclear fission reactors," Engineering Failure Analysis, Vol. 18, Escola Politecnica do Brazil, 1943 (2011).

[24] M. FINLAY, G. HOFMAN, and J. SNELGROVE, "Irradiation behavior of uranium silicide compounds," Journal of Nuclear Materials, 325, 118 (2004).

[25] J. REST and G. HOFMAN, "Dynamics of irradiation-induced grain subdivision and swelling in $\mathrm{U}_{3} \mathrm{Si}_{2}$ and $\mathrm{UO}_{2}$ fuels," Journal of Nuclear Materials, 210, 187 (1994). 
[26] G. HOFMAN and W. RYU, "Detailed analysis of uranium silicide dispersion fuel swelling," Proceedings of the RERTR International Meeting, Berlin, Germany, September 10-13, 1989, p. 10 (1989).

[27] M PLANCK and A. OGG, Treatise on Thermodynamics, Dover Publications, New York (1945).

[28] J. HONIG, Thermodynamics, Fourth Edition: Principles Characterizing Physical and Chemical Processes, Elsevier, Amsterdam (2007).

[29] N. HALL, (National Aeronautics Space Administration), Second Law of Thermodynamics, http://www.grc.nasa.gov/www/k-12/airplane/thermo2.html, Accessed (Dec. 2017).

[30] E. FERMIN, Thermodynamics, Dover Publications, New York (1956).

[31] F. CAMPBELL, Phase Diagrams: Understanding the Basics, ASM International, Ohio (2012).

[32] D. JOHNSON, and G. STRACHER, Thermodynamic Loop Applications in Materials Systems, Minerals, Metals \& Materials Society, Warrendale, Pennsylvania (1995).

[33] W. CALLISTER and D. RETHWISCH, Fundamentals of Materials Science and Engineering: an Integrated Approach, Wiley, Hoboken, New Jersey (2008).

[34] D. PORTER, K. EASTERLING, and M. SHERIF, Phase Transformations in Metals and Alloys, CRC Press, London (2009).

[35] S. KAKANI and A. KAKANI, Material Science, New Age International, New Delhi (2004).

[36] M. CHUN and K. ROSE, "Interaction of High-Intensity Laser Beams with Metals," Journal of Applied Physics, 41, 614 (1970).

[37] D. BERGSTROM, J. POWELL, and A. KAPLAN, "A ray-tracing analysis of the absorption of light by smooth and rough metal surfaces," Journal of Applied Physics, 101, 113504 (2007).

[38] K. TANG, R. DIMENNA, and R. BUCKIUS, "Regions of validity of the geometric optics approximation for angular scattering from very rough surfaces," International Journal of Heat and Mass Transfer, 40, 49 (1996).

[39] B. QIAN and Z. SHEN, "Laser sintering of ceramics," Journal of Asian Ceramic Societies, 1, 315 (2013).

[40] A. MUNITZ, A. GOKHALE, and G. ABBASCHIAN, "The Ce-Si (Cerium-Silicon) System," Bulletin of Alloy Phase Diagrams, 10, 73 (1989). 
[41] H. OKAMOTO, “The Si-Zr (silicon-zirconium) system," Journal of Phase Equilibria, 11, 513 (1990).

[42] A. GOKHALE, and G. ABBASCHIAN, "The Hf-Si (hafnium-silicon) system," Bulletin of Alloy Phase Diagrams, 10, 390 (1989).

[43] J. ROSALES, I. VAN ROOYEN, S. MEHER, R. HOGAN, C. PARGA, and J. HARP, ""Effect of High Si Content on $\mathrm{U}_{3} \mathrm{Si}_{2}$ Fuel Microstructure," JOM, 70, 209 (2018).

[44] M. LE FLEM, J. CANEL, and S. URVOY, "Processing and characterization of $\mathrm{Zr}_{3} \mathrm{Si}_{2}$ for nuclear applications," Journal of Alloys and Compounds, 465, 269 (2008).

[45] C. JOURNEAU, J. MONERRISA, B. TORMOS, L. BRISSONNEAU, E. EXCOFFIER, V. TESTUDB, C. CHAGNOT, and D. ROULET, "Fabricating Fukushima Daiichi invessel and ex-vessel fuel debris simulants for the development and qualification of laser cutting technique." European Review Meeting on Severe Accident Research, Warsaw, Poland, May 16-18, 2017, p. 11 (2017).

[46] P. CHEVREUX, A. LAPLACE, E. DELEULE, L. TISSANDIER and N. MASSONI, "Hafnium solubility determination in soda-lime aluminosilicate glass," Journal of NonCrystalline Solids, 457, 13 (2017).

[47] P. EDMONSON, Y. ZHANG, S. MOLL, F. NAVAMAR, and W. WEBER, "Irradiation effects on microstructure change in nanocrystalline ceria-Phase, lattice stress, grain size and boundaries," Acta Materialia, 60, 5408 (2012).

[48] B. HEMINGWAY, Thermodynamic properties of selected uranium compounds and aqueous species at $298.15 \mathrm{~K}$ and 1 bar and at higher temperatures; preliminary models for the origin of coffinite deposits, No. 82-619, U.S. Geological Survey (1982).

[49] G. INGMAR, J. FUGER, R. KONINGS, R. LEMIRE, A. MULLER, C. NGUYENTRUNG, and H. WANNER, Chemical Thermodynamics of Uranium Vol. 1, Nuclear Energy Agency, Amsterdam (1992).

[50] M. FERNANDEZ-PEREA, J. AZNAREZ, J. LARRUQUERT, J. MENDEZ, L. POLETTO, D. GARACOLI, A. MALVEZZI, A. GIGLIA, and S. NANNARONE, "Transmittance and optical constants of Ce films in the 6-1200 eV spectral range," Journal of Applied Physics, 103, 073501 (2008).

[51] W. SIEKHAUS, and A. NELSON, "The optical properties of a polished uranium surface and its epitaxial oxide, and the rate of oxide growth determined by spectrophotometry," MRS Online Proceedings Library Archive, 893 (2005).

[52] C. CUTHBERTSON and E. PRIDEAUX, "On the refractive index of gaseous fluorine," In Proc. R. Soc. Lond. A, 76, 426 (1905).

[53] S. COTTON, Lanthanide and actinide chemistry, John Wiley \& Sons, England (2013). 
[54] G. DITTMER, and U. NIEMANN, "Evaluation of thermodynamic data on zirconium and hafnium halides and oxyhalides by means of transport experiments," Philips Journal of Research, 42, 15 (1987).

[55] L. TOPOR and O. KLEPPA, "Standard enthalpies of formation of $\mathrm{Me}_{5} \mathrm{Si}_{3}(\mathrm{Me} \equiv \mathrm{Y}, \mathrm{Lu}$, $\mathrm{Zr}$ ) and of $\mathrm{Hf}_{3} \mathrm{Si}_{2}$, , Journal of the Less Common Metals, 167, 99 (1990).

[56] B. SOBOLEV, The Rare Earth Trifluorides: The high temperature chemistry of the rare earth trifluorides, Vol. 124, Institut d'Estudis Catalans, Spain (2000).

[57] G. KRITHIGA, L. DOMBROVSKY, T. OH and W. LIPINSKI, "Determination of optical constants of ceria by combined analytical and experimental approaches," JOM, 65, 1694 (2013).

[58] E. GOHARSHADI, S. SAMIEE, and P NANCARROW. "Fabrication of cerium oxide nanoparticles: characterization and optical properties." Journal of colloid and interface science, 356, 473 (2011).

[59] T. OH, Y. TOKPANOV, Y HAO, W. JUNG, and S. HAILE, "Determination of optical and microstructural parameters of ceria films," Journal of Applied Physics, 112, 103535 (2012).

[60] M. AFANASIEV, S. HABUDA, and A. LUNDIN, "The symmetry and basic structures of $\mathrm{LaF}_{3}, \mathrm{CeF}_{3}, \mathrm{PrF}_{3}$ and $\mathrm{NdF}_{3}$," Acta Crystallographica Section B: Structural Crystallography and Crystal Chemistry, 28, 2903, (1972).

[61] E. WESTRUM, and A. BEALE, "Heat Capacities and Chemical Thermodynamics of Cerium (III) Fluoride and of Cerium (IV) Oxide From 5 to $300^{\circ} \mathrm{K}$," The Journal of Physical Chemistry, 65, 353 (1961).

[62] M. MADOU, T. OTAGAWA and A. SHER, "Solid compositions for fuel cell electrolytes," U.S. Patent 4,948,680, (Aug. 1990).

[63] W. LYON, D. OSBORNE, H. FLOTOW, F. GRANDJEAN, W. HUBBARD, and G. JOHNSON, "Thermodynamics of the lanthanide trifluorides. I. The heat capacity of lanthanum trifluoride, $\mathrm{LaF}_{3}$ from 5 to $350 \mathrm{~K}$ and enthalpies from 298 to 1,477 K," The Journal of Chemical Physics, 69, 167 (1978).

[64] K. HANSEN, C. RUNGAROONTHAIKUL, T. AMINABHAVI, and C. YAWS, "Gaseous Thermal Conductivity of Silane, Dichlorosilane, Trichlorosilane, Tetrachlorosilane, and Tetrafluorosilane in the Temperature Range from 28 to 350 degrees C" Journal of Chemical and Engineering Data, 40, 15 (1995).

[65] K. GSCHNEIDNER, L. EYRING, and G. LANDER, Handbook on the Physics and Chemistry of Rare Earths, Vol. 32, Elsevier, Holland (2002). 
[66] L. SILVERSTONI, D. SCITI, J. KLING, S. LAUTERBACH, and H. KLEEBE, "Sintering mechanisms of zirconium and hafnium carbides doped with $\mathrm{MoSi}_{2}$," Journal of the American Ceramic Society, 92, 1574 (2009).

[67] O. KLEPPA and S. WATANABE, "Thermochemistry of Alloys of Transition Metals: Part III. Copper-silver-titanium, and hafnium at 1373 K," Journal of Electronic Materials, 20, 391 (1991).

[68] D. ZIPPERIAN, Metallographic Handbook, Pace Technologies, Tucson, Arizona (2011).

[69] J. HANET, "Phase explosion induced by high repetition rate pulsed laser," Applied Surface Science, 1, 6649, (2010).

[70] J. BELCHER, C. HANSON, H. BERATAN, K. UDAYAKUMAR, and K. SOCH, "SPIE Process," Infrared Technology Applications, Vol. 3436, 611 (1998).

[71] P. SCHAAF, Laser Processing Materials, Springer, New York (2010).

[72] F. GUITTONEAU, A. ABDELOUAS, B. GRAMBOW and M. DIALINAS, "New Methods for HTR Fuel Waste Management," Proceedings of the 4th International Topical Meeting on High Temperature Reactor Technology, Washington DC, September 28October 1, 2008 p. 116 (2008)

[73] ASTM E1245-03, "Standard Practice for Determining the Inclusion or Second-Phase Constituent Content of Metals by Automatic Image Analysis," ASTM International, West Conshohocken, Pennsylvania (2016).

[74] G. PRASAD, V. SINHA, and P. HEGDE, "Development and Fabrication of LEU Plate Fuel for Modified Core of APSARA Reactor,” BARC Newsletter, 21 (2012).

[75] L. HE, J. HARP, R. HOGGAN, and A. WAGNER "Microstructure studies of interdiffusion behavior of $\mathrm{U}_{3} \mathrm{Si}_{2} /$ Zircaloy-4 at 800 and $1,000^{\circ} \mathrm{C}$," Journal of Nuclear Materials, 486, 274 (2017).

[76] A. LEENAERS, "Post-irradiation examination of AlFeNi cladded $\mathrm{U}_{3} \mathrm{Si}_{2}$ fuel plates irradiated under severe conditions," Journal of Nuclear Materials, 375, 243 (2008).

[77] J. CARMACK, “Thermal Properties Measurement Report,” INL/EXT-15-36283, Idaho National Laboratory (2015).

[78] U.S. Department of Energy, "Development of Light Water Reactor Fuels with Enhanced Accident Tolerance," Report to Congress, Washington DC (2015).

[79] A. KAUFMANN, B. CULlity, G. BITSIANES, P. GORDON, M. COHEN, and R. BOSTIAN, “The Chemistry of Uranium," Part I, National Nuclear Energy Series, Div. VIII, Vol. 5, McGraw-Hill, New York (1951). 


\section{BIOGRAPHICAL SKETCH}

Jhonathan Rosales war born in Guatemala City, Guatemala in 1,988. He received his Bachelor of Science in mathematics with a minor in physics from Wisconsin Lutheran College in May of 2011. He started working for CREE Inc. as a lighting engineer from July 2011 to August of 2013. In the fall of 2013 he enrolled in the Nuclear Engineering program at the University of Florida and earned a Master of Science degree in May of 2015. He continued his doctoral research at the Idaho National Laboratory under mentorship of Dr. Isabella van Rooyen. At INL, he initially worked on the development of a database of microstructural and physical properties on candidate fuels for the Transient Reactor Test (TREAT) reactor fuel conversion program. He also was involved in the High Temperature Gas Reactor (HTGR) fuel development program and contributed with mechanistic studies on the fission product deposition on the SiC layer of tristructural isotropic (TRISO) fuel. His main project at INL was the characterization activities of the Additive Manufacturing as an Alternate Fabrication Technique (AMAFT) for uranium silicide $\left(\mathrm{U}_{3} \mathrm{Si}_{2}\right)$ fuel. The characterization along the different development stages of the AMAFT project was his doctoral dissertation topic and he earned his Doctor of Philosophy degree from the University of Florida in May of 2018. 